\title{
Insights into mitochondrial presequence and carrier import pathways
}

\author{
DISSERTATION \\ for the award of the degree \\ "Doctor rerum naturalium" \\ of the Georg-August-Universität Göttingen \\ within the doctoral program Molecular Biology \\ of the Georg-August University School of Science (GAUSS)
}

\author{
submitted by \\ Ridhima Gomkale \\ from New Delhi, India
}

Göttingen, September 2018 


\section{Members of the Thesis Committee}

Prof. Dr. Peter Rehling

(Supervisor and first referee)

Prof. Dr. Holger Stark

(Second referee)

Prof. Dr. Patrick Cramer
Institute of Cellular Biochemistry

University Medical Center Göttingen

Germany

Department of Structural Dynamics

Max Planck Institute for Biophysical Chemistry Göttingen, Germany

Department of Molecular Biology

Max Planck Institute for Biophysical Chemistry

Göttingen, Germany

\section{Additional Members of the Examination Board}

Prof. Dr. Henning Urlaub

Prof. Dr. Stefan Jakobs

Dr. Alexander Stein
Bioanalytical Mass Spectrometry

Max Planck Institute for Biophysical Chemistry

Göttingen, Germany

Structure and Dynamics of Mitochondria Max Planck Institute for Biophysical Chemistry Göttingen, Germany

Membrane Protein Biochemistry Max Planck Institute for Biophysical Chemistry Göttingen, Germany

Date of oral examination: 12.11.2018 


\section{Affidavit}

I hereby declare that my dissertation, entitled 'Insights into mitochondrial presequence and carrier import pathways', has been written independently and with no other tools or sources than quoted.

Ridhima Gomkale

Göttingen, September 2018

Parts of this thesis are communicated in the following publication:

Gomkale, R.*, Cruz-Zaragoza, L.D.*, Suppanz, I., Guiard, B., Montoya, J., Callegari, S., Pacheu-Grau, D., Warscheid, B. and Peter Rehling. "Defining the Substrate Spectrum of the TIM22 Complex Identifies Pyruvate Carrier Subunits as Unconventional Cargos" Current Biology 30(6) (2020).

Other results of the project will be communicated in the following publication:

Gomkale, R., Linden, A., Neumann, P., Stoldt, S., Schendzielorz, A.B., Ficner, R., Jakobs, J., Urlaub, H. and Rehling, P. "Modelling precursor translocation at the TOMTIM23 interface" In preparation (2020).

*denotes equal contribution 


\section{Acknowledgements}

First and foremost, a heartfelt gratitude to my supervisor Prof. Dr. Peter Rehling. The four years in the lab were an excellent time to work on many stimulating projects. I am grateful to him for his continuous support, motivation and guidance regarding my PhD study.

I am grateful to Prof. Dr. Holger Stark for taking out the time for discussion on many occasions. Special thanks also to Prof. Dr. Patrick Cramer for being a member of my thesis committee and sharing his knowledge. Thank you also to Prof. Dr. Henning Urlaub, Prof. Dr. Stefan Jakobs and Dr. Alexander Stein for taking out time to be a member of my extended committee.

A warm thanks to Dr. Christian Schulz for making me look at research in a different light and inspiring me through his patience and supervision to be a better scientist.

This study would not have been possible without collaborative efforts. I would like to thank Dr. Niels Fischer for the analysis of our electron microscopy samples, and Andreas Linden for cross-linking experiments. They always remained optimistic and shared their technical knowledge freely. Also, many thanks to Dr. Alexander Stein for providing protease constructs, Dr. Ida Suppanz for mass spectrometry analysis and Dr. Stefan Stoldt for super-resolution microscopy. I would like to acknowledge Klaus, Robert and Olaf for providing technical assistance with protein purification.

A very special gratitude goes to Dr. Steffen Burkhardt and Kerstin Grüniger from the IMPRS Molecular Biology program for their constant support. It's a privilege to be a part of the program!

A big thank you to all the current and former members of the department. I would like to make a special mention of Alex and David, not only for giving critical and thoughtful scientific feedback, but also for being amazing friends outside of lab. The four years together were an adventure. Additionally, I am grateful to Tobi for being my eternal cheerleader in the last four years, Cong for all the discussions about gradient centrifugation and good food, Frank for his steady and reliable presence, Natasha for her scientific comments, suggestions and a healthy dose of crazyness. I am also thankful to Sylvie and Markus for always being open to scientific discussions regarding my queries. Thanks to Abhishek, Arpita, Bettina, Carmen, Christine, Florian, Isotta, Kathi, Lioba, Luis, 
Magnus, Mandy, Mariia, Martina, Mirjam, Roya, Sabine and Sven for creating an excellent and friendly working atmosphere.

I am also thankful to the students who I got the opportunity to supervise.

Friends are the family we choose and life in Göttingen wouldn't have been the same without them. I am very grateful to my support system of Priyanka, Paridhi and Vedran for their constant encouragement and sympathy, and with whom I have created many happy memories. A warm thank you also to Alice, Kashish, Marc, Kanika, Arshiya and Ika for being good friends.

Last and most importantly, I would like to thank my parents for believing in me and instilling in me the spirit to approach my work with integrity, and my siblings, whose excellence in all aspects of their life is a source of inspiration. Finally, my niece, for keeping me grounded. 
ADAPT AND OVERCOME. 


\section{Contents}

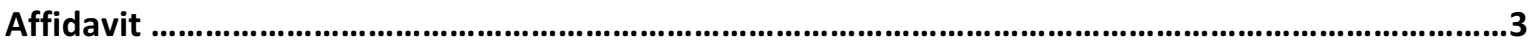

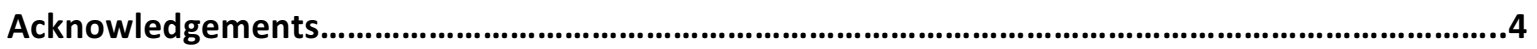

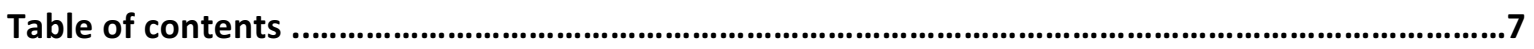

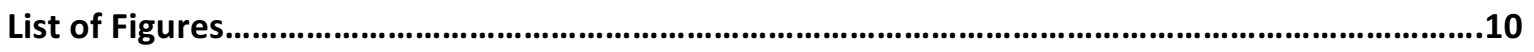

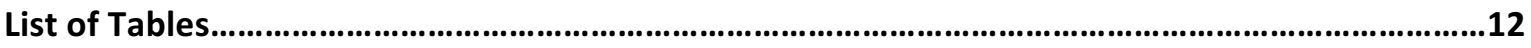

List of abbreviations.........................................................................................................................13

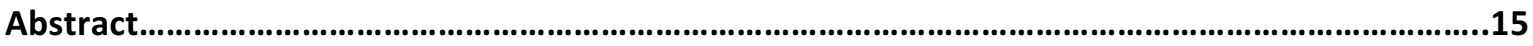

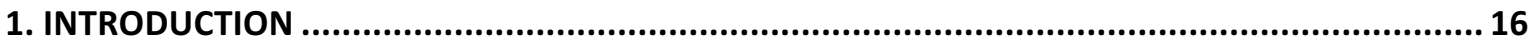

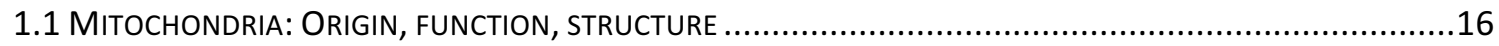

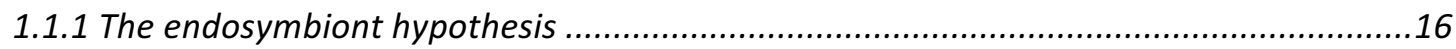

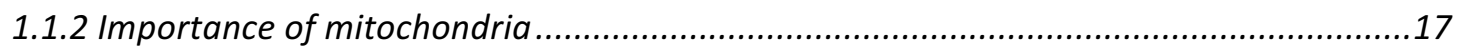

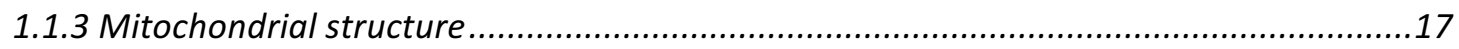

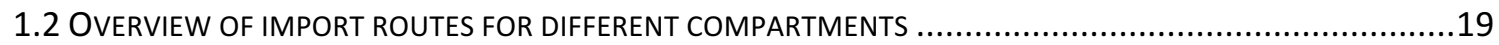

1.2.1 Translocase of the Outer Mitochondrial Membrane: the TOM complex .......................21

1.2.2 Protein import into the inner membrane via the TIM22 complex: The carrier pathway 23

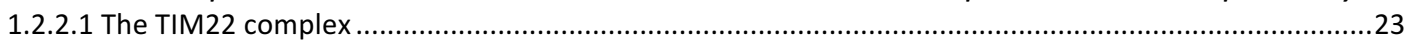

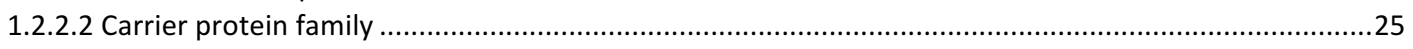

1.2.3 Protein import via the TIM23 complex: The presequence pathway .............................26

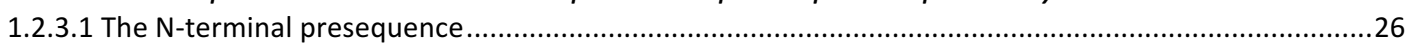

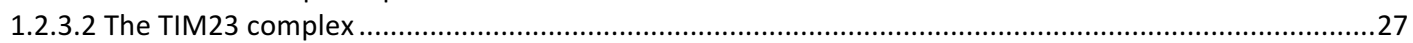

1.2.3.3 The Presequence translocase associated motor (PAM) complex.....................................................31

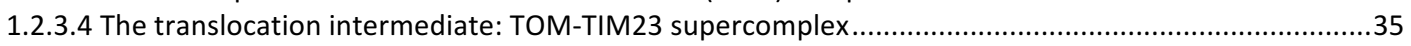

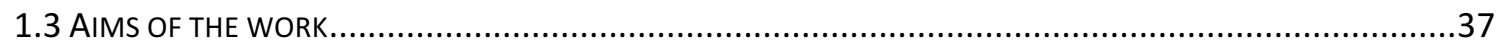

2. MATERIALS AND METHODS

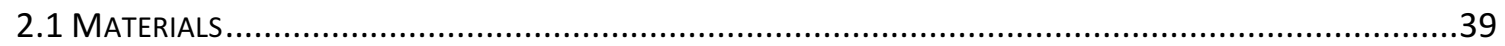

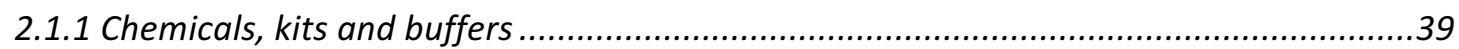

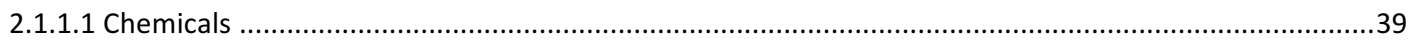

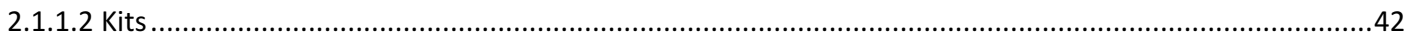

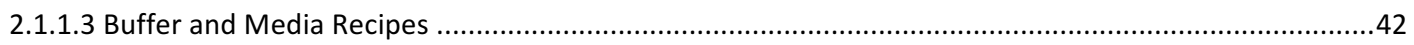

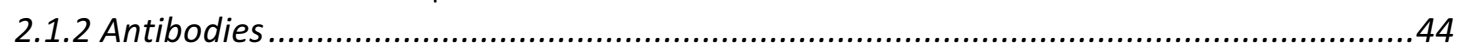

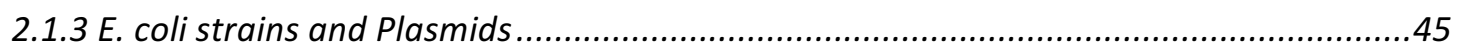

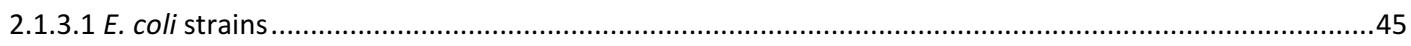

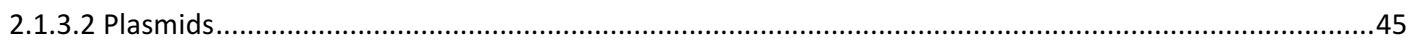

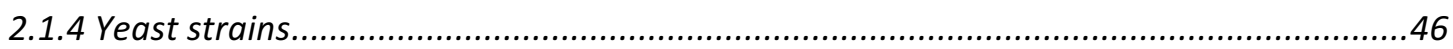

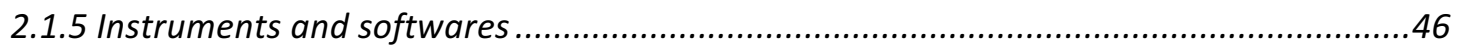

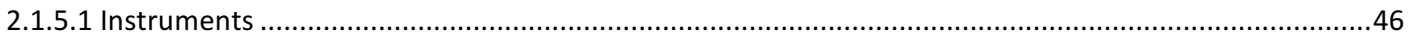

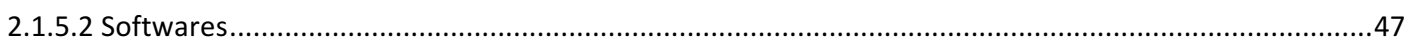

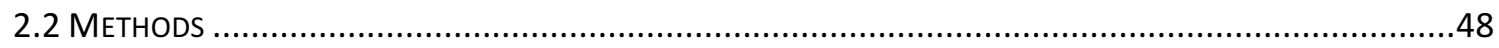

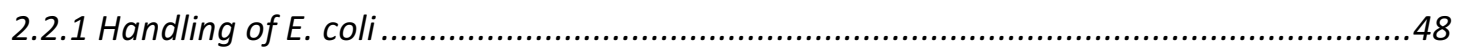

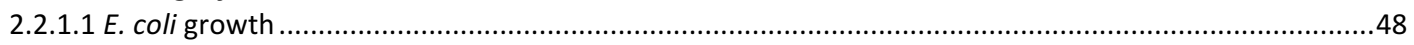

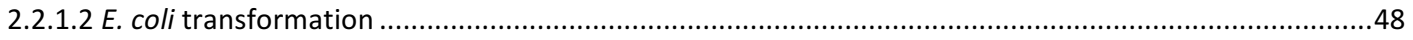

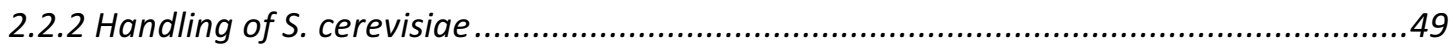

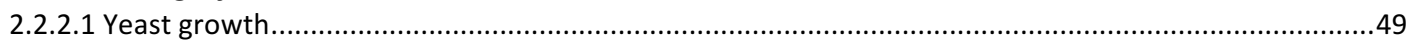

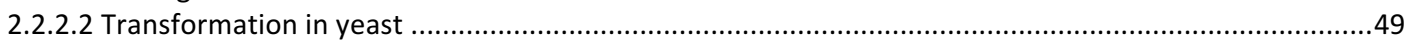

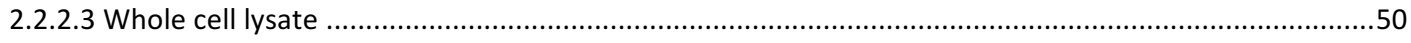

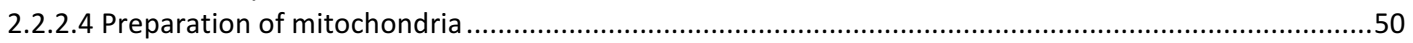

2.2.2.5 Microscopy of yeast cells ........................................................................................................51

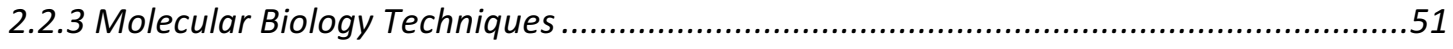

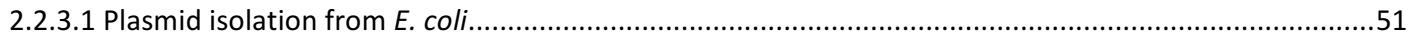

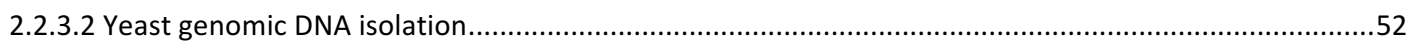

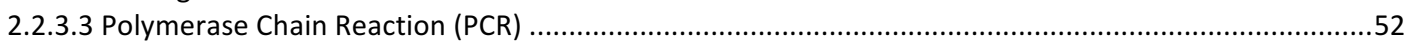

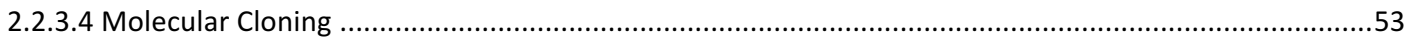

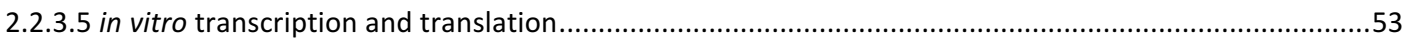




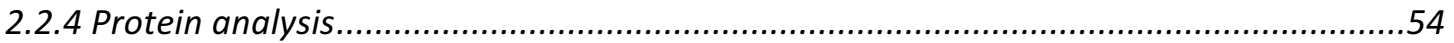

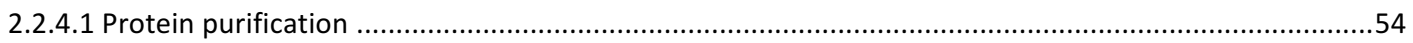

2.2.4.2 SDS-PAGE

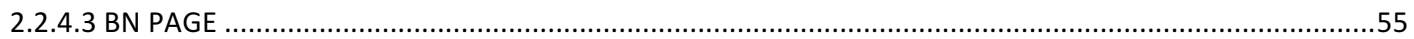

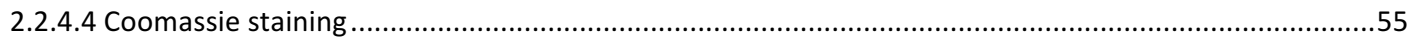

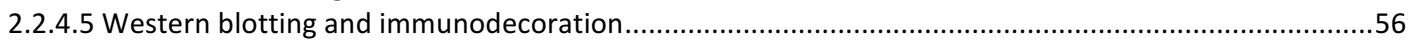

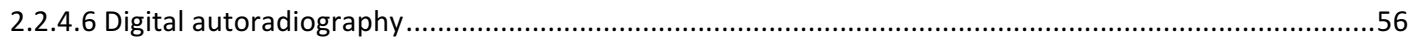

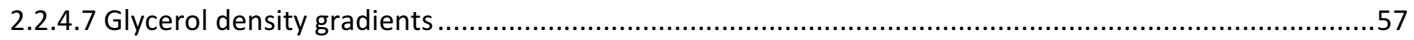

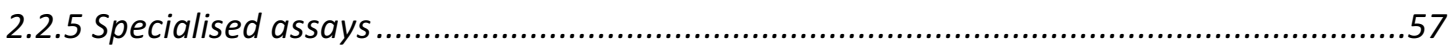

2.2.5.1 Protein import, assembly and generation of the TOM-TIM23 supercomplex ....................................57

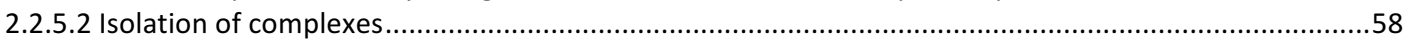

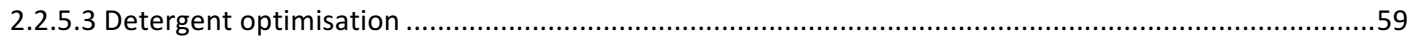

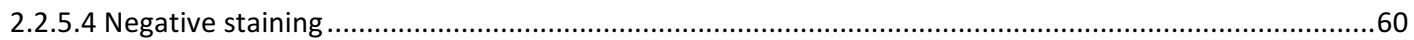

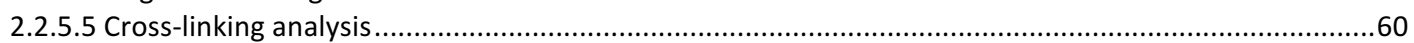

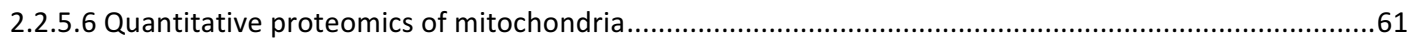

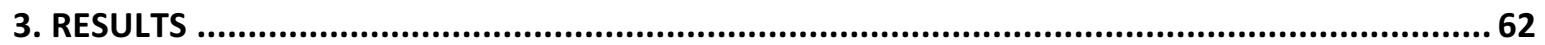

3.1 ANALYSIS OF A MITOCHONDRIAL TRANSLOCATION INTERMEDIATE: THE TOM-TIM23 SUPERCOMPLEX ......62

3.1.1 Design and comparison of new supercomplex forming proteins.............................62

3.1.1.1 in vivo expression of protein shows localisation to mitochondria .......................................................63

3.1.1.2 New fusion proteins form the supercomplex to varying levels .......................................................65

3.1.1.3 Accumulation of supercomplex forming proteins at the translocase inhibits import of mitochondrial

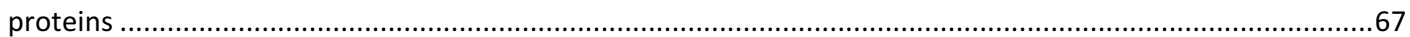

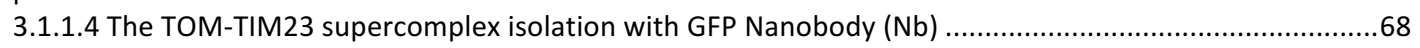

3.1.2 Optimisation of supercomplex isolation conditions.........................................70

3.1.2.1 SUMOstar and Nedd8 tags on Tim23 are suitable for native isolation of the TIM23 complex..............70

3.1.2.2 Optimisation of solubilisation conditions for the TIM23 complex purification ....................................72

3.1.2.3 Glycerol density gradient separation of the supercomplex ................................................................74

3.1.2.4 Chemical fixation with glutaraldehyde during the gradient run (GraFix) ..........................................76

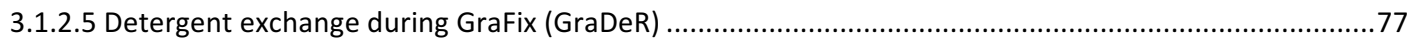

3.1.3 Structural analysis of the isolated TIM23 complex and the TOM-TIM23 supercomplex 78

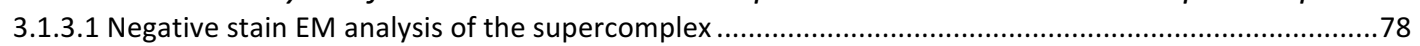

3.1.3.2 Cross-linking mass spectrometry analysis to define protein dynamics in the TIM23 complex and the

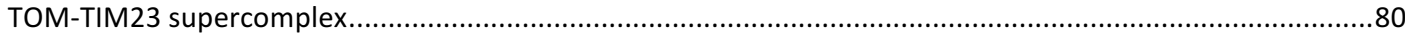

3.2 EXPANSION OF SUBSTRATE SPECTRUM OF THE TIM22 COMPLEX ...............................................89

3.2.1 Characterisation of the Tim22 temperature sensitive strain .......................................90

3.2.1.1 Selection of Tim22 temperature sensitive strain ..........................................................................90

3.2.1.2 Temperature induced mislocalisation of known TIM22 complex substrates in the tim22-14 strain .......91

3.2.2 Mitochondrial inner membrane carrier and transporter proteins are specifically affected in tim 22-14 mitochondria under non-permissive conditions ..........................................93

3.2.2.1 Mass spectrometric analysis to identify carrier substrates ..............................................................93

3.2.2.2 Steady-state analysis of mitochondrial proteins after heat shock ....................................................96

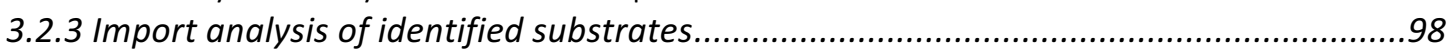

3.2.3.1 TIM23 complex substrate import is not affected in tim22-14 mitochondria ......................................98

3.2.3.2 Assembly of carrier proteins is affected in tim 22-14 mitochondria ...................................................99

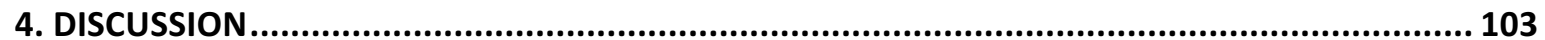

4.1 ANALYSIS OF A MITOCHONDRIAL TRANSLOCATION INTERMEDIATE: THE TOM-TIM23 SUPERCOMPLEX ....103

4.1.1 Supercomplex forming proteins as a tool to study active protein import in mitochondria

4.1.2 Optimisation of the TOM-TIM23 supercomplex isolation conditions ........................104

4.1.3 Cross-linking analyses of the mitochondrial TOM-TIM23 supercomplex...................106

4.1.3.1 Tim21 interacts with Tom22 in the presence of a translocation arrested protein...............................106

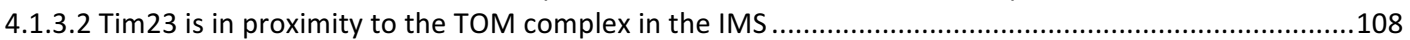

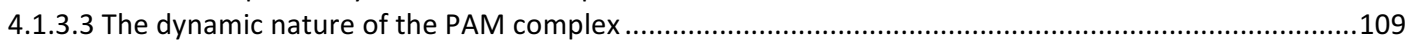

4.2 EXPANSION OF SUBSTRATE SPECTRUM OF THE TIM22 COMPLEX ..........................................111

4.2.1 Mitochondrial carrier proteins are depleted in tim 22-14 .....................................111

4.2.2 Turnover rate of Tim23 and Tim17 is low .......................................................113

4.2.3 Offbeat import pathways into mitochondria ................................................113 
5. SUMMARY AND FUTURE PERSPECTIVES

6. Bibliography..

Curriculum vitae..

.135 


\section{List of Figures}

Figure 1 Scheme of mitochondrial structure ………...................................... 18

Figure 2 Major import routes for mitochondrial proteins ................................. 19

Figure 3 Translocase of the outer mitochondrial membrane (TOM) ............... 21

Figure 4 Transport along the carrier pathway ……………………………...... 23

Figure 5 Different forms of the TIM23 complex in yeast .................................... 28

Figure 6 Components of the PAM complex..................................................... 33

Figure 7 TOM-TIM23 supercomplex formation .................................................. 36

Figure 8 Design of supercomplex forming proteins ......................................... 63

Figure 9 Supercomplex forming proteins localise to mitochondria ................ 64

Figure 10 Super-resolution microscopy of Jac1-sfGFP ...................................6 65

Figure 11 Purification of supercomplex forming proteins shows tendency to

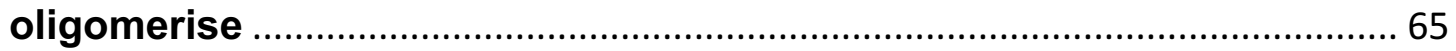

Figure 12 Chemical amounts of SCGP and Jac1-sfGFP form supercomplex 66

Figure 13 Supercomplex forming proteins block presequence import........... 68

Figure 14 The TOM-TIM23 supercomplex can be isolated using GFP Nb....... 69

Figure 15 His-SUMOstar and His-Nedd8 tag on Tim23 helps in specific isolation of the TIM23 complex .................................................................. 71

Figure $16 \mathrm{GDN}$ is an alternative detergent that can be used for supercomplex isolation

Figure 17 Isolated TIM23 complex and supercomplex on glycerol gradients 75

Figure 18 Chemical fixation during gradient centrifugation stabilises the

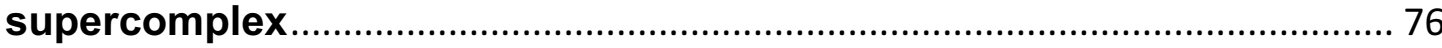

Figure 19 Detergent exchange during gradient centrifugation stabilises the

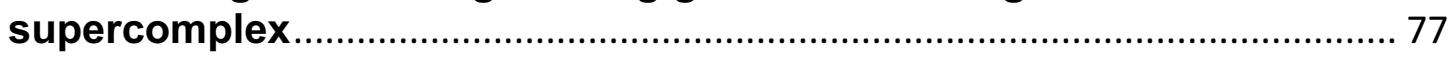

Figure 20 Representative negative stain images ………………….............. 79

Figure 21 Comparison of different cross-linkers on isolated TOM-TIM23

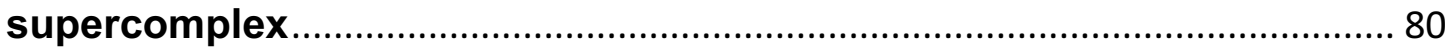

Figure 22 Fold change in peptide spectrum matches of TOM, TIM23 and TIM22 complex components

ure 23 Visualisation of cross-links obtained in the absence and presence of Jac1-sfGFP

Figure 24 Tim50-Tim21 cross-links in the absence and presence of Jac1-

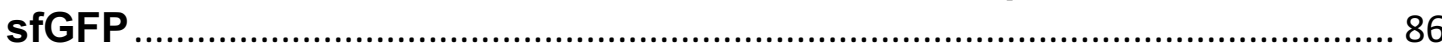

Figure 25 Tim21 cross-links with Tom22 in the presence of Jac1-sfGFP....... 87

Figure 26 PAM complex subunits undergo intra- and inter-protein crosslinking

Figure 27 tim22-14 shows proper growth and Tim22 levels at permissive temperature

Figure 28 Carrier proteins (AAC2 and Mir1) mislocalise from mitochondria in the tim22-14 strain under repressive growth conditions .......................... 92

Figure 29 Mixing scheme for WT and tim22-14 mitochondria under different conditions.

Figure 30 t-test overview of protein levels in tim22-14 and WT mitochondria after heat stress

Figure 31 TIM22 complex substrate levels specifically decrease in tim22-14 mitochondria upon heat stress 
Figure 32 Import of TIM23 complex substrates not affected in tim22-14

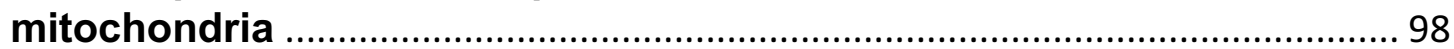

Figure 33 Assembly of carrier proteins affected in tim22-14 mitochondria. 100 Figure 34 Assembly of uncharacterised carrier protein YFR045W affected in tim22-14 mitochondria 101

Figure 35 Assembly of uncharacterised protein YPR011C reduced in tim2214 mitochondria 101 


\section{List of Tables}

Table 1 Components of the TOM complex.......................................................... 22

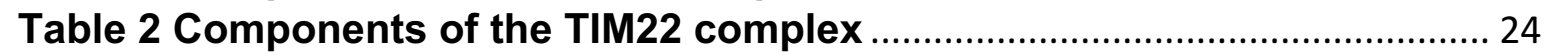

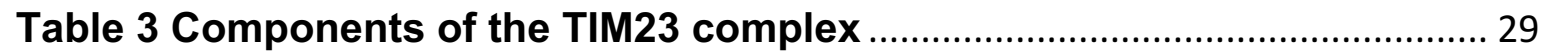

Table 4 Components of the PAM complex....................................................... 31

Table 5 List of chemicals used in this study and their suppliers ................... 39

Table 6 List of commercial kits used in this study along with their

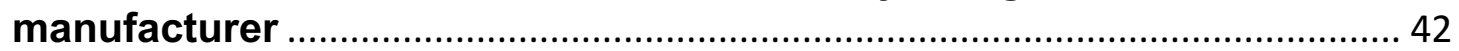

Table 7 List of commonly used buffers in this study along with their

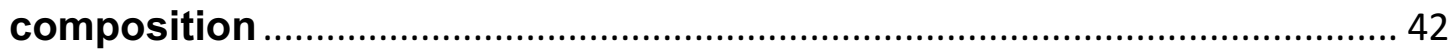

Table $8 \mathrm{E}$. coli strains used in this study along with their genotype ............... 45

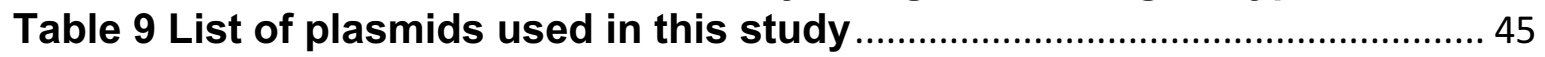

Table 10 S. cerevisiae strains used in this study along with their genotype. 46

Table 11 List of laboratory equipments used in this study along with their

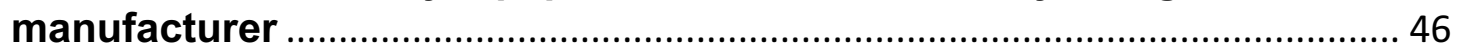

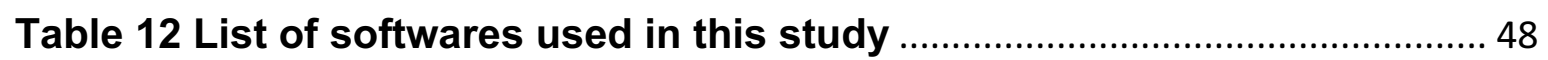

Table 13 Summary of different supercomplex-forming proteins tested ........ 70

Table 14 Carrier and transporter proteins most affected in tim22-14 ............ 95 


\section{List of Abbreviations}

\begin{tabular}{|c|c|}
\hline AAC & ADP/ATP carrier \\
\hline APS & Ammonium persulfate \\
\hline ATP & Adenosine triphosphate \\
\hline AVO & Antimycin $\mathrm{A}$, valinomycin, oligomycin mixture \\
\hline BN-PAGE & Blue native polyacrylamide gel electrophoresis \\
\hline BS2G & Bissulfosuccinimidyl glutarate \\
\hline BS3 & Bissulfosuccinimidyl suberate \\
\hline BSA & Bovine serum albumin \\
\hline CK & Creatine kinase \\
\hline $\mathrm{CP}$ & Creatine phosphate \\
\hline Cryo-EM & Cryo electron microscopy \\
\hline CS & Carrier signature \\
\hline CTD & C-terminal domain of Tim44 \\
\hline $\mathrm{dH}_{2} \mathrm{O}$ & Deionized water \\
\hline DHFR & Mouse dihydrofolate reductase \\
\hline DMSO & Dimethyl sulfoxide \\
\hline DNA & Deoxyribonucleic acid \\
\hline dNTP & 2'-deoxynucleoside-5'-triphosphate \\
\hline DSG & Disuccinimidyl gluterate \\
\hline DSS & Disuccinimidyl suberate \\
\hline E. coli & Escherichia coli \\
\hline ECL & Enhanced chemiluminescence \\
\hline EDTA & Ethylenediaminetetraacetic acid \\
\hline EM & Electron microscopy \\
\hline EtBr & Ethidium bromide \\
\hline GDN & Glyco diosgenin \\
\hline GFP & Green Fluorescent Protein \\
\hline HEPES & 4-(2-hydroxyethyl)-1-piperazineethanesulfonic acid \\
\hline HRP & Horse radish peroxidase \\
\hline $\mathrm{IM}$ & Inner mitochondrial membrane \\
\hline IMS & Intermembrane space \\
\hline IPTG & Isopropyl- $\beta$-D-thiogalactopyranoside \\
\hline $\mathrm{kDa}$ & Kilodalton \\
\hline LB & Lysogeny broth \\
\hline LMNG & Lauryl Maltose Neopentyl Glycol \\
\hline MCP & Mitochondrial carrier and transporter proteins \\
\hline MOPS & Morpholinopropanesulfonic acid \\
\hline MPP & Mitochondrial processing peptidase \\
\hline MS & Mass spectrometry \\
\hline MTS & Mitochondrial targeting signal \\
\hline MTX & Methotrexate \\
\hline $\mathrm{NADH}$ & Nicotinamide adenine dinucleotide (reduced) \\
\hline $\mathrm{Nb}$ & Nanobody \\
\hline NBD & Nucleotide binding domain of Ssc1 \\
\hline NTD & N-terminal domain of Tim44 \\
\hline OD & Optical density \\
\hline $\mathrm{OM}$ & Outer mitochondrial membrane \\
\hline PAGE & Polyacrylamide gel electrophoresis \\
\hline PAM & Presequence translocase associated motor \\
\hline
\end{tabular}




\begin{tabular}{||l|l|}
\hline PBD & Presequence binding domain \\
\hline PBS & Phosphate buffer saline \\
\hline PCR & Polymerase chain reaction \\
\hline PEG & Polyethylene glycol \\
\hline PK & Proteinase K \\
\hline PLD & Protein loading dye \\
\hline PMSF & Phenylmethylsulfonylfluoride \\
\hline PVDF & Polyvinylidene fluoride \\
\hline S. cerevisiae & Saccharomyces cerevisiae \\
\hline SBD & Substrate binding domain of Ssc1 \\
\hline SCGP & SuperComplex Generating Protein \\
\hline SD-Ura & Selective glucose media minus Uracil \\
\hline SDS & Sodium dodecyl sulfate \\
\hline SfGFP & superfolder GFP \\
\hline SMA & Styrene:Maleic anhydride \\
\hline SSuC-Leu & Selective sucrose media minus Leucine \\
\hline TAE & Tris/Acetate/EDTA buffer \\
\hline TBS & Tris buffer saline \\
\hline TCA & Trichloroacetic acid \\
\hline TEMED & N,N,N',N'-tetramethylethylenediamine \\
\hline TIM22 & Carrier translocase of the inner mitochondrial membrane \\
\hline TIM23 & Presequence translocase of the inner mitochondrial membrane \\
\hline TOM & Translocase of the outer mitochondrial membrane \\
\hline ts & temperature sensitive \\
\hline V/V & Volume/volume \\
\hline w/v & Weight/volume \\
\hline WT & Wild type \\
\hline XL & Cross-linker \\
\hline YPAD & YPD with adenine \\
\hline YPD & Yeast extract, peptone, glucose \\
\hline YPG & Yeast extract, peptone, glycerol \\
\hline$\Delta \Psi$ & Membrane potential \\
\hline & \\
\hline & \\
\hline
\end{tabular}




\section{Abstract}

The mitochondria of yeast Saccharomyces cerevisiae contains more than 1000 proteins, majority of which are imported from the cytosol. The TIM23 and the TIM22 complexes in the inner mitochondrial membrane are essential transport systems for proper insertion of inner membrane and matrix proteins in mitochondria. Additionally, both complexes recognise different targeting signals on a precursor protein. In this study, the TIM23 and the TIM22 complexes were investigated regarding their structure and substrate spectrum respectively.

The TOM and the TIM23 complexes cooperate for importing presequence-containing proteins into mitochondria. However, the lack of structural information of the TIM23 complex prevents us from completely deciphering the exact mechanism for the import of a presequence-containing substrate. In this study, we designed and generated new proteins for formation of the TOM-TIM23 supercomplex in organello and in vivo. Subsequently, we optimised the isolation strategy to obtain preparatory amounts of the supercomplex for structural and cross-linking analysis. Through our cross-linking analysis, we mapped the interaction between subunits of the TIM23 complex in its unoccupied state and its translocation intermediate TOM-TIM23 supercomplex state. We identified cross-links between Tim23-Tom40, Tim21-Mgr2 and Hsp70-Mge1 in the unoccupied TIM23 complex. Additionally, cross-links between Tom22-Tim21, Pam16-Tim44 and Pam16-Pam18 were also identified in the supercomplex state of TIM23. Together, these suggest the dynamic nature of interactions within the subunits of the PAM complex, as well as between TOM and TIM23 subunits, during the process of translocation of a protein into mitochondria.

The TIM22 complex is required for the import of polytopic inner membrane proteins which lack a presequence but have internal targeting signals. These proteins have predominantly been defined to contain either four or six transmembrane domains. However, so far, only a few proteins have been identified as being substrates of this complex. Therefore, the primary aim of the second project was to expand the substrate spectrum of the TIM22 complex. For this, we utilised a Tim22 temperature sensitive strain in combination with quantitative mass spectrometry. Numerous proteins belonging to the carrier family, such as Crc1, Odc1, Yhm2 and Hem25, were confirmed as substrates of the TIM22 complex. Moreover, previously uncharacterised proteins YPR011C and YFR045W were also identified as TIM22 substrates.

Together, these results expand our knowledge about the molecular interactions between mitochondrial translocase components during active protein import, as well as increase our repertoire of the TIM22 complex substrates. 


\section{Introduction}

\subsection{Mitochondria: Origin, function, structure}

\subsubsection{The endosymbiont hypothesis}

Darwin's principles of natural selection govern most of the biological diversity today. But can microscopic events, such as the origin of mitochondria and chloroplast in present day eukaryotic cells, also be explained by them? This question is in part answered by the endosymbiotic theory, which states that organelles, which are hallmarks of eukaryotic cells, evolved as symbionts within early prokaryotic cells. The presence of DNA and active translation system within these organelles supports the endosymbiont hypothesis (Margulis,1970). In agreement to this, organelle DNA is usually found in the form of circular molecules, similar to that found in bacteria (Timmis et al., 2004). Detailed analysis of the mitochondrial genome has confirmed the bacterial origin of mitochondria (Gray et al., 1984) (Gray et al., 1989), whereas mitochondrial rRNA evolutionary trees showed that they evolved from the $\alpha$-class of proteobacteria (Yang et al., 1985), specifically from the Rickettsia species (Andersson et al., 1998) (Gray, 1998). However, a recent study suggested that mitochondria and Rickettsias evolved from two independent endosymbiotic events (Martijn et al., 2018).

Endosymbiotic gene transfer (EGT) has led to the transfer of many essential genes from the genome of the endosymbiont to that of the host, leading to a reduced organelle genome size. This also necessitates transport systems within the organelle to re-import the nuclear-encoded proteins from the cytosol (Keeling and Archibald, 2008) (Martin et al., 2001). The endosymbiont, in return, provided energy required by the host. Therefore, the host and the organelle have co-evolved over time to function as one entity. Upon division of the host cell, mitochondria also needed to divide and be distributed to each daughter cell. The mechanism of formation of new mitochondria is similar to that observed for bacteria, i.e. through binary fission (Margolin, 2005). Constant fission and fusion events are required to maintain the interconnected and dynamic mitochondrial network within the cell. 


\subsubsection{Importance of mitochondria}

Mitochondria are essential organelles for the cell and are involved in diverse cellular functions. They are predominantly known for their role in the generation of ATP. The coupling of ATP synthesis to the electrochemical gradient across the inner mitochondrial membrane was proposed in the chemiosmotic theory (Mitchell, 1961). Accordingly, the sequential passage of electrons along the respiratory chain complexes (four enzyme complexes in the mitochondrial inner membrane, termed complex I-IV) leads to the formation of a proton gradient, which is used to generate ATP by ATP synthase (complex $\mathrm{V})$ in the mitochondrial matrix (Saraste, 1999).

Mitochondria are also known for their role in various metabolic pathways, including heme biosynthesis, iron sulphur protein biogenesis (Lill et al., 2012), fatty acid and lipid metabolism, the tricarboxylic acid (TCA) cycle, amino acid metabolism and the urea cycle. They are also involved in important regulatory aspects of the cell, including calcium signalling (Clapham, 2007), apoptosis (programmed cell death) (Green and Reed, 1998) and ROS signalling (Shadel and Horvath, 2015). Therefore, due to their prominent role in energy production, mitochondria dysfunction can lead to diseases. Defects in mitochondrial DNA have been implicated in diseases like Leigh syndrome and Leber's hereditary optic neuropathy (LHON) (Osellame et al., 2012).

\subsubsection{Mitochondrial structure}

The biogenesis of mitochondria is critical for homeostatic functioning of the cell. In most eukaryotes, mitochondria are present as a connected network (Friedman and Nunnari, 2014) and not as single entities. Endosymbiosis has led to mitochondria having two membranes. The outer mitochondrial membrane (OM) is similar to the bacterial host membrane in its lipid composition, whereas the inner membrane (IM) retains the properties of the symbiont organism membrane. Within them, the two membranes enclose the aqueous intermembrane space (IMS) and the matrix (Figure 1).

The mitochondrial outer membrane acts as a barrier for macromolecules, while allowing the diffusion of small ions and metabolites (O'Brien and Brierley, 1965) (Benz, 1994). This is facilitated by the $\beta$-barrel protein porin/VDAC (voltage-dependent anion channel). The permeable nature of the outer membrane results in a similar ionic composition of the cytosol and IMS. Recently, however, this view was challenged with the identification of new channel proteins which were selective for anions or cations, implicating the outer membrane in a more regulatory role of metabolite flux (Krüger et al., 2017).

The mitochondrial inner membrane has a larger surface area and more complex architecture compared to the outer membrane. The passage of molecules across this 
membrane is tightly regulated, since it is highly impermeable in nature. This is required to maintain the electrochemical gradient across the membrane, which is generated and utilised by the oxidative phosphorylation system.

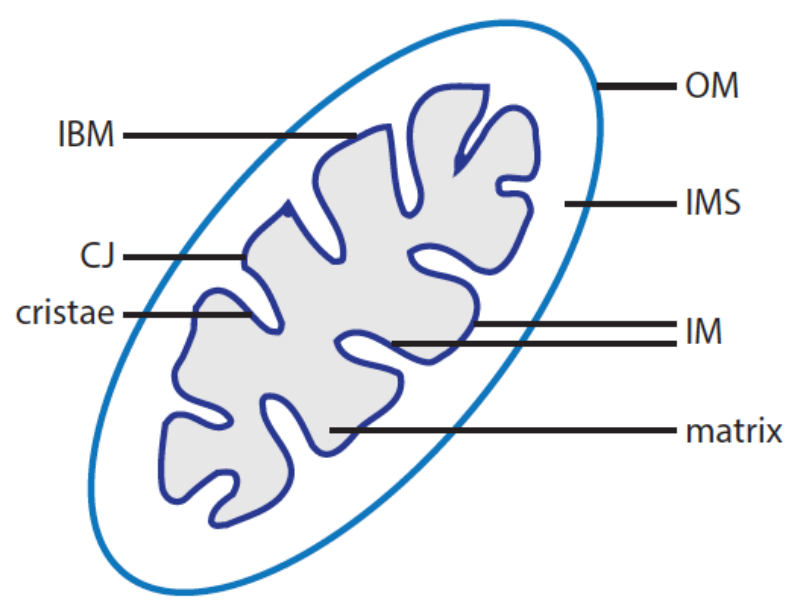

Figure 1 Scheme of mitochondrial structure - The mitochondrial outer membrane (OM) and inner membrane (IM) enclose the intermembrane space (IMS) between them. The inner membrane can be present as inner boundary membrane (IBM), cristae junction (CJ) or as cristae invaginations into the matrix.

The inner membrane can be structurally and functionally subcompartmentalised into different domains (Figure 1). These are: (i) Inner boundary membrane (IBM), (ii) cristae junction (CJ) and (iii) cristae. The IBM is a stretch of inner membrane in close proximity to the outer membrane. Such positioning of the two membranes is thought to facilitate the import of proteins into the organelle via dedicated protein complexes (Vogel et al., 2006). Extended invaginations of the inner membrane into the matrix are termed cristae. Cristae junctions (CJ) form the connection between the IBM and the cristae. Both the $\mathrm{CJ}$ and cristae are rich in specific protein sets, which are responsible for their curvature. CJ are stabilised by MICOS (Mitochondrial contact site and Cristae Organising System) (Hoppins et al., 2011) (Malsburg et al., 2011) (Harner et al., 2011) (Alkhaja et al., 2012) (van der Laan et al., 2012), whose subunits induce curvature of the membrane. The presence of $F_{1}$ $F_{0}$-ATP synthase dimers, as well as components of the respiratory chain complexes are essential for cristae morphology (Paumard et al., 2002). These complexes can oligomerise and stabilise the cristae. Specific mutants of the $F_{1} F_{0}-A T P$ synthase show reduced membrane potential and cristae formation (Alkhaja et al., 2012) (Bornhövd et al., 2006).

Taken together, the subcompartmentalisation of the inner membrane and mitochondria as a whole, as well as the gene transfer of most mitochondrial proteins to the nucleus, entails the need for a protein import machinery specific for the different compartments. 


\subsection{Overview of import routes for different compartments}

The mitochondria of yeast Saccharomyces cerevisiae contain about 1000 proteins, as has been previously established by proteomic studies (Sickmann et al., 2003), genome-wide high throughput localisation screens (Huh et al., 2003), protein functional interaction network analysis (Perocchi et al., 2006) and more recently, quantitative mass spectrometry (Morgenstern et al., 2017) analysis. Approximately $99 \%$ of these proteins have to be imported from the cytosol (Chacinska et al., 2009) (Becker et al., 2012) and are targeted to the four different compartments. Initial in vitro studies demonstrated the presence of a signal sequence on the precursor mitochondrial protein, which determines its final destination to mitochondria (Neupert and Schatz, 1981). Over time, a plethora of cleavable and non-cleavable targeting and sorting signals have been identified (Neupert and Herrmann, 2007) (Chacinska et al., 2009) (Endo et al., 2011). Dedicated import machineries are present in mitochondria to ensure correct targeting of precursor proteins (Figure 2) and to maintain mitochondrial function and dynamics.

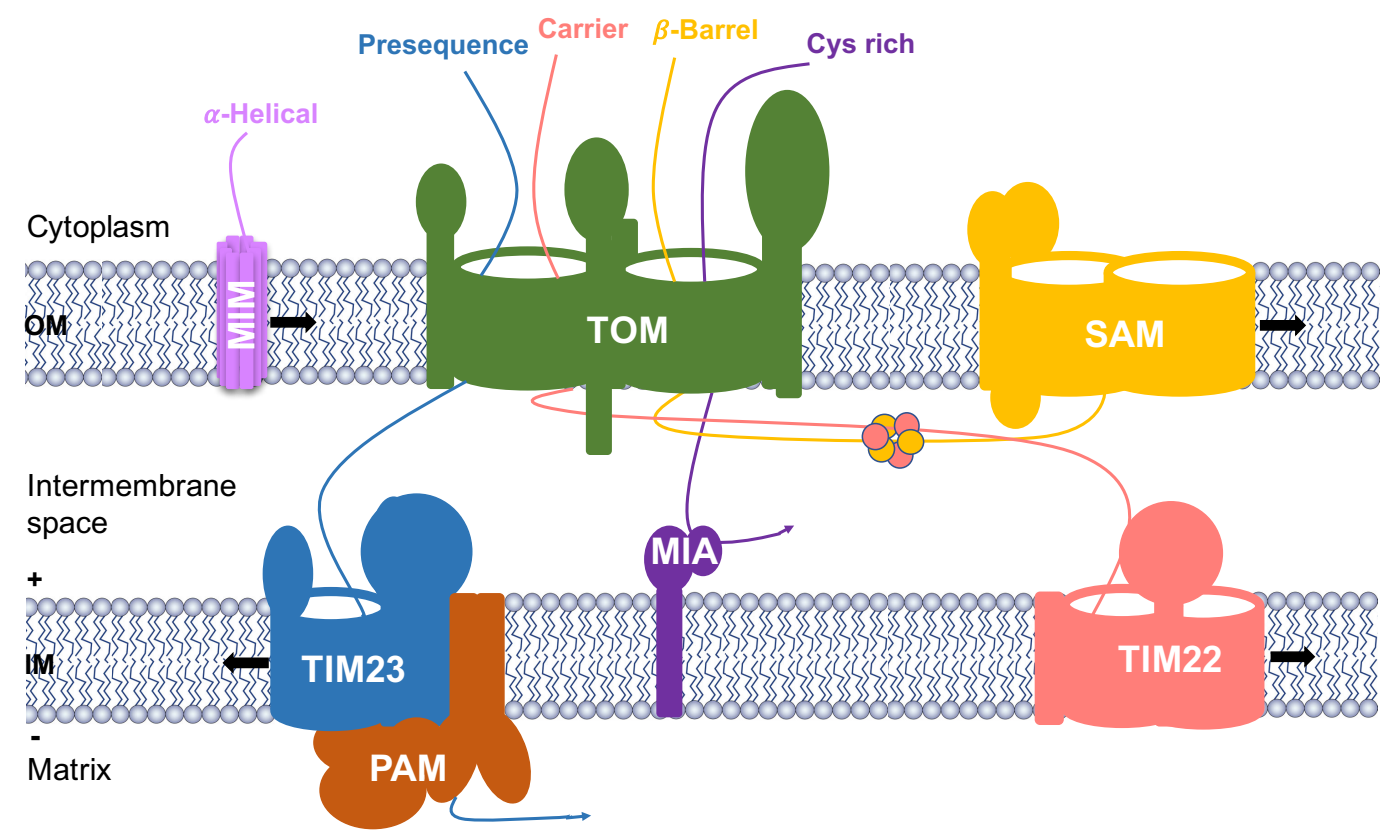

Figure 2 Major import routes for mitochondrial proteins - Mitochondrial precursor proteins can be subdivided into five major groups. Majority of these proteins enter mitochondria through the translocase of the outer mitochondrial membrane (TOM). Presequence containing proteins are recognised by the translocase of the inner mitochondrial membrane (TIM23), and are either sorted into the inner mitochondrial membrane or transported into the matrix with the help of the presequence translocase-associated motor (PAM) complex. Carrier proteins containing internal hydrophobic signals, as well as $\beta$-barrel proteins, associate with the small TIM chaperones in the IMS. They are inserted into the IM and OM by the TIM22 and SAM (sorting and assembly machinery) complexes respectively. Cysteine rich proteins associate with the MIA (mitochondrial IMS import and assembly) system in the IMS. Some $\alpha$-helical OM proteins are inserted via the mitochondrial import (MIM) complex. 
Newly synthesised mitochondrial proteins usually associate with cytosolic chaperones of the Hsp family to remain in an unfolded state (Young et al., 2003) (Jores et al., 2018). The major import routes for these proteins are:

1) Presequence pathway: Almost $2 / 3^{\text {rds }}$ of mitochondrial proteins have a cleavable presequence at the $\mathrm{N}$-terminus. This is an amphipathic $\alpha$-helix, with positively charged amino acids on one face of the helix and hydrophobic ones on the other. Presequencecontaining proteins are recognised by receptors of the TOM (translocase of the outer membrane) complex and subsequently the TIM23 (translocase of the inner membrane) complex, after which they are sorted into the inner membrane or imported into the matrix. The PAM (presequence translocase associated motor) complex is required for localisation to matrix. The presequence is cleaved by the MPP (mitochondrial processing peptidase) (Schatz and Dobberstein, 1996) (Vögtle et al., 2009) (Schulz et al., 2015) in the matrix.

2) Carrier pathway: This pathway utilises non-cleavable internal signals within multispanning inner membrane proteins. Upon entry through the TOM complex, small TIM chaperones in the IMS bind and stabilise the carrier pathway substrate proteins, followed by their import into the inner membrane by the TIM22 complex (Sirrenberg et al., 1996) (Kerscher et al., 1997) (Koehler et al., 1998a) (Sirrenberg et al., 1998).

3) $\beta$-barrel pathway: Outer membrane $\beta$-barrel precursor proteins are imported by the TOM complex. In the IMS, they associate with small TIM chaperones. The SAM (sorting and assembly machinery) complex then inserts them into the outer membrane (Wiedemann et al., 2003) (Paschen et al., 2003).

4) Cysteine rich IMS proteins: Mitochondrial IMS proteins containing specific cysteine motifs are imported by the TOM complex. In the IMS, they associate with MIA (mitochondrial intermembrane space import and assembly) machinery which promotes their oxidation and folding (Naoé et al., 2004) (Chacinska et al., 2004) (Terziyska et al., 2005).

5) $\alpha$-helical OM proteins: Only a few outer membrane proteins with $\alpha$-helical transmembrane segments are recognised by the MIM (mitochondrial import) complex, which also promotes their insertion (Becker et al., 2008) (Hulett et al., 2008) (PopovCeleketić et al., 2008).

However, non-conventional import routes are still being discovered, indicating that specific import machineries cater to precursor specific requirements. These, along with import via the presequence and carrier pathway, will be discussed in detail in the following sections. 


\subsubsection{Translocase of the Outer Mitochondrial Membrane: the TOM complex}

The majority of mitochondrial proteins are imported via the TOM complex (Figure 3). The major components of this complex are: (i) the $\beta$-barrel channel-forming protein Tom40 (Kiebler et al., 1990) (Lackey et al., 2014), which consists of 19 TM $\beta$ strands (Shiota et al., 2015). It is also referred to as the general import pore (GIP); (ii) Receptor $\alpha$-helical proteins, Tom20, Tom22 and Tom70 (Brix et al., 1997). Tom20 is the initial presequence receptor, whose cytosolic domain binds the hydrophobic surface of the presequence (Abe et al., 2000). Tom22, on the other hand, is the central receptor, which binds to the positively charged surface of the presequence. It also has a soluble presequence binding domain in the IMS, which helps in the transfer of proteins from the TOM to the TIM23 complex (Shiota et al., 2011). Moreover, Tom22 is essential for the oligomerisation of the TOM complex (van Wilpe et al., 1999). Tom70 acts as the primary receptor for hydrophobic proteins (Schlossmann et al., 1994). Its cytosolic domain interacts with Hsp70 family proteins bound to the precursors. Recently, the role of Tom70 in presequence binding has also been demonstrated (Melin et al., 2015), as well as its ability to recognise iMTS-Ls \{internal MTS (matrix targeting signal)-like signals\} (Backes et al., 2018); (iii) small Tom proteins Tom5, Tom6 and Tom7. These proteins are involved in the TOM complex assembly, stability and dynamics (Wiedemann and Pfanner, 2017). Tom6 was observed to stabilise the interaction between Tom40 and Tom22 (Alconada et al., 1995), whereas Tom5 was reported to act as a link during transfer of precursor proteins from TOM receptors to the GIP (Dietmeier et al., 1997). A summary of the functions of different TOM complex components is mentioned in Table 1.

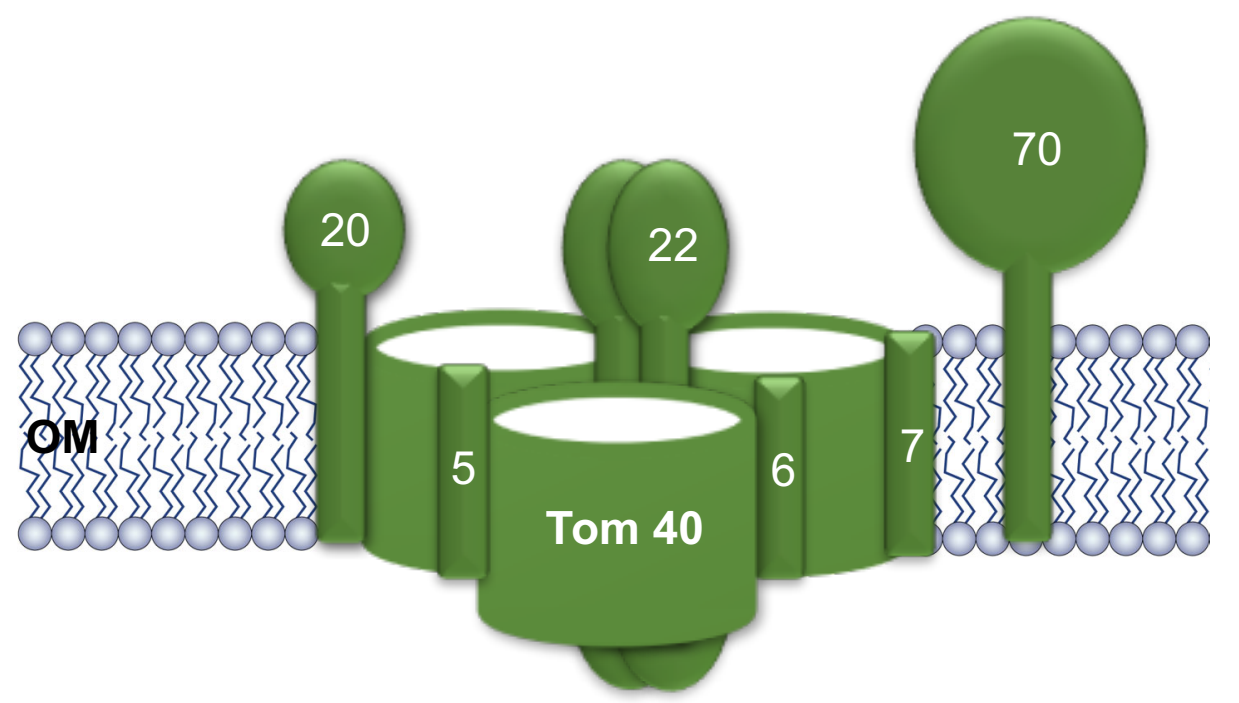

Figure 3 Translocase of the outer mitochondrial membrane (TOM) - The mitochondrial TOM complex is made up of seven subunits. The general import pore Tom40 is the main entry gate for proteins. Tom20, Tom22 and Tom70 act as receptors for different classes of preproteins. Small proteins Tom5, Tom6 and Tom7 promote assembly and stabilisation of the complex. 
Table 1 Components of the TOM complex. Different subunits of the TOM complex along with their known functions.

\begin{tabular}{|l|l|}
\hline Protein & Function \\
\hline Tom40 & Channel-forming $\beta$-barrel protein \\
\hline Tom20 & Initial receptor for presequence-containing proteins \\
\hline Tom22 & Central receptor, involved in TOM complex oligomerisation \\
\hline Tom70 & Receptor for non-cleavable hydrophobic proteins \\
\hline Tom71 & Tom70 paralog \\
\hline Tom5 & TOM complex assembly, transfer of precursor proteins to Tom40 \\
\hline Tom6 & TOM complex assembly and dynamics \\
\hline Tom7 & TOM complex disassembly and dynamics \\
\hline
\end{tabular}

Structurally, the TOM complex has been reported as a two or three pore-containing complex (Model et al., 2008) (Bausewein et al., 2017) (Figure 3). Tom40 is the protein that forms the pore. It has also been shown to associate with translocating proteins and prevent their aggregation (Esaki et al., 2003). In Neurospora crassa, two Tom40 proteins associate with the small Toms and with Tom22 to form the $148 \mathrm{kDa}$ TOM-core complex (TOM-CC). Cryo-electron microscopy analysis of this complex pointed to a double symmetric molecule of Tom40 dimers, stabilised by Tom22 transmembrane domains (Bausewein et al., 2017). Transmembrane densities of Tom5, Tom6 and Tom7 were also present around Tom40. For $S$. cerevisiae, the $550 \mathrm{kDa}$ Tom20-core (TOM-CC with Tom20) complex was structurally analysed (Model et al., 2008). It had a near three-fold symmetry, with three pores being identified, along with three Tom22 subunits. Crosslinking studies in S. cerevisiae indicated that the mature trimeric TOM complex consists of three Tom40 proteins tethered by Tom22 transmembrane segments, along with Tom20, Tom5, Tom6 and Tom7 (Shiota et al., 2015). This form of the complex is in a dynamic exchange process with a Tom22-free dimeric form, which can act as an assembly intermediate. From the same study, Tom40 was also shown to have specific hydrophobic and acidic amino acid patches in its channel, for transport of carrier and presequence proteins.

To summarise, Tom22 and Tom20 recognise different regions of the amphipathic presequence simultaneously. With the help of Tom5, these precursor proteins are guided from the Tom22-Tom20 subcomplex to the Tom40 pore. The IMS domain of Tom22 helps in the transfer of the preprotein to downstream import complexes. 


\subsubsection{Protein import into the inner membrane via the TIM22 complex: The carrier pathway}

\subsubsection{The TIM22 complex}

Import of a subset of multi-spanning inner membrane hydrophobic proteins is mediated by the TIM22 complex. This twin-pore containing complex is made up of the integral membrane channel-forming protein Tim22, the single membrane-spanning Tim54 with a large IMS domain, Tim18 and Sdh3 (Dudek et al., 2013). Small Tim IMS proteins Tim8, Tim9, Tim10 and Tim13, as well as the peripherally attached Tim12, are also involved in import via this pathway (Table 2). Known substrates of the TIM22 complex include four transmembrane-spanning translocase core components Tim23, Tim22 and Tim17, as well as six transmembrane carrier proteins such as the ADP/ATP carrier (AAC), the phosphate carrier $(\mathrm{PiC})$ and the dicarboxylate carrier (DiC).

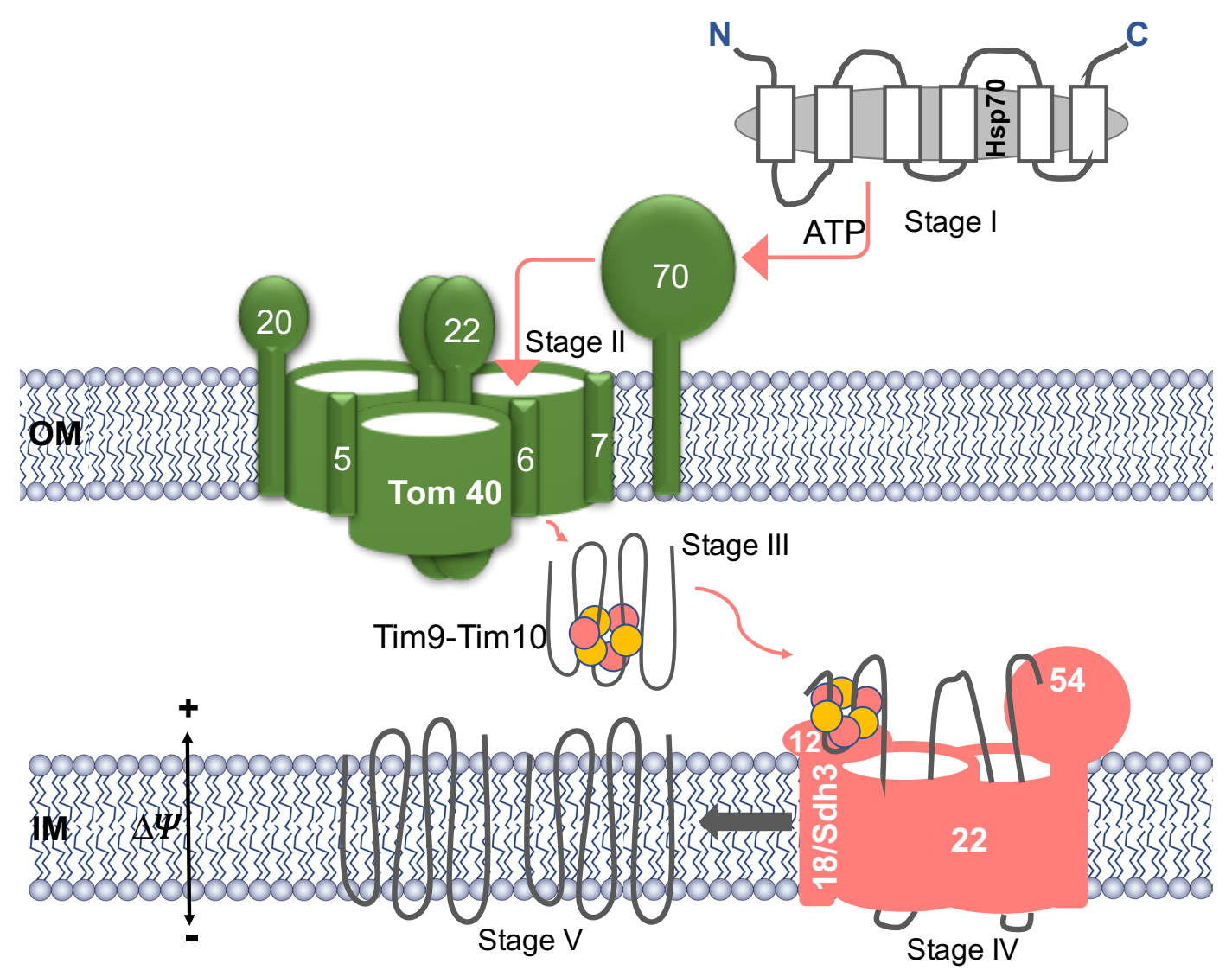

Figure 4 Transport along the carrier pathway - Hydrophobic proteins with internal signals are kept in an unfolded state by Hsp chaperones in the cytosol (stage I). After being recognised by their primary receptor Tom70, the proteins translocate through the TOM complex (stage II). In the IMS, small Tim hexamer of Tim9 and Tim10 associates with the peptide (stage III) and guides it to the TIM22 complex. A membrane potential dependent insertion by TIM22 (stage IV) is followed by assembly of the protein (stage $\mathrm{V}$ ). $\Delta \Psi$ : membrane potential. 
Tim22 is the channel-forming core subunit of the $300 \mathrm{kDa}$ TIM22 complex (Sirrenberg et al., 1996) (Kovermann et al., 2002) (Rehling et al., 2003). It is an integral membrane protein, which has four transmembrane domains and shares a strong sequence similarity to Tim23 and Tim17. Tim54 is a membrane protein with a domain in the IMS, which binds to the Tim9-Tim10-Tim12 complex (Kerscher et al., 1997) (Wagner et al., 2008). Tim18 is a non-essential protein involved in the assembly of Tim54 (Kerscher et al., 2000) (Koehler et al., 2000) (Wagner et al., 2008). Another protein which was surprisingly discovered to be a part of the TIM22 complex is Sdh3 (Gebert et al., 2011). This protein is also a subunit of respiratory chain complex II, and is involved in the assembly and stability of Tim18 and therefore the TIM22 complex.

The small Tims are a family of proteins involved in mitochondrial import. These are Tim9, Tim10, Tim8, Tim13 and Tim12 and they act as chaperones in the IMS by associating with hydrophobic proteins and transferring them to their destination. Tim9 and Tim10 form a heterohexameric complex, which interacts with hydrophobic proteins as they exit the TOM complex (Sirrenberg et al., 1998) (Koehler et al., 1998a) (Koehler et al., 1998b) (Adam et al., 1999). Another essential protein, Tim12, associates with this hexamer and docks it to the TIM22 complex (Gebert et al., 2008). Therefore, Tim12 links the soluble and membrane-associated components of this import pathway. The Tim9-Tim10 hexamer also mediates the transfer of proteins from the TOM complex to the SAM complex (Wiedemann et al., 2004). Tim8 and Tim13 are non-essential small Tim proteins that form a heterohexamer. These are involved in the transfer of a subset of inner membrane proteins such as Tim23 (Koehler et al., 1999) (Paschen et al., 2000) (Curran et al., 2002).

Table 2 Components of the TIM22 complex. Subunits of the TIM22 complex and the associated IMS chaperones with their functions.

\begin{tabular}{|l|l|}
\hline Protein & Function \\
\hline Tim22 & Channel-forming subunit \\
\hline Tim54 & Binding platform for Tim9-Tim10-Tim12 complex \\
\hline Tim18 & Tim54 assembly \\
\hline Sdh3 & Assembly of TIM22 complex \\
\hline Tim9-Tim10 & IMS chaperones for hydrophobic proteins \\
\hline Tim8-Tim13 & IMS chaperones for subset of inner membrane proteins \\
\hline Tim12 & Tether for IMS chaperones at the TIM22 complex \\
\hline
\end{tabular}




\subsubsection{Carrier protein family}

Mitochondrial carrier and transporter proteins (MCPs) are a group of inner membrane proteins encoded by the nuclear DNA. They are responsible for shuttling a variety of charged or hydrophilic solutes across the inner membrane. MCPs don't have an Nterminal presequence, but they have several internal targeting signals instead (Saraste and Walker, 1982). The typical MCP structure consists of three tandem repeat modules (module I-III) of about 100 amino acids, with each repeat containing two transmembrane helices connected by hydrophilic loops, as was first reported for the AAC structure (Kunji and Harding, 2003) (Pebay-Peyroula et al., 2003). The N- and C-termini are in the IMS. The first helix of each repeat has a characteristic conserved sequence at its C-terminal end, $\operatorname{PX}(D / E) X X(K / R)$, called the carrier signature (CS) (Kunji, 2004) (Nelson et al., 1998) (Belenkiy et al., 2000) (Ferramosca and Zara, 2013). Genomic studies have identified 35 proteins belonging to the MCP group in yeast (Nelson et al., 1998). However, to date, only a few of these have been characterised in terms of their import and function. Among the most commonly studied carrier proteins are the ADP/ATP carrier (AAC), the phosphate carrier $(\mathrm{PiC})$ and the dicarboxylate carrier $(\mathrm{DiC})$. These have also been identified as substrates of the TIM22 complex through import analysis (Sirrenberg et al., 1996). The role of different MCP modules was studied to identify the targeting information in them. Module III by itself (Brandner et al., 2005), or in combination with module II (Pfanner et al., 1987b) (Endres et al., 1999) has been shown to be important for associating with the TIM22 complex for DiC and AAC respectively. Therefore, a broader substrate spectrum is required to completely elucidate the roles of different modules.

Transport of carrier proteins across the outer membrane and into the inner membrane is a well-studied process. It consists of five distinct stages (Figure 4) (Rehling et al., 2004). Carrier proteins synthesised in the cytosol are highly hydrophobic in nature. Chaperones in the cytosol, Hsp70 and Hsp90, bind to the precursor proteins post-translationally to prevent their aggregation (stage I) (Young et al., 2003). The chaperone-bound precursor binds at specific sites on Tom70 (stage II) (Wu and Sha, 2006). The three modules of the precursor then bind to three Tom70 dimers (Wiedemann et al., 2001). Chaperone release from the precursor is facilitated by ATP (Ryan et al., 1999), after which the carrier proteins traverse the TOM complex pore in a loop formation, such that the $\mathrm{N}$ - and $\mathrm{C}$ - termini remains in the cytosol while the middle part enters the channel (Söllner et al., 1992) (Curran et al., 2002). At the trans side of the TOM complex, the Tim9-Tim10 complex associates with the importing precursor (stage III). In the IMS, Tim9-Tim10 act as chaperones for the hydrophobic precursor and associate with Tim12 to dock together at 
the TIM22 complex (Sirrenberg et al., 1998) (Koehler et al., 1998a) (Koehler et al., 1998b). The membrane potential $(\Delta \psi)$ is required for the next two stages as a driving force for insertion of protein into the inner membrane. Proteins are inserted into the TIM22 complex (stage IV), from which they are laterally released into the inner membrane (Rehling et al., 2003). Here they assemble to form a mature complex (stage $V$ ).

Although the import mechanism of these proteins is understood to some extent, only a few carrier proteins have been shown to be imported via this pathway. Therefore, there is a need to comprehensively analyse and broaden the substrate spectrum of the TIM22 complex.

\subsubsection{Protein import via the TIM23 complex: The presequence pathway}

\subsubsection{The $\mathrm{N}$-terminal presequence}

A comprehensive N-proteome study by Vögtle et al demonstrated that majority of the mitochondrial proteins $(70 \%)$ utilise an $\mathrm{N}$-terminal presequence for their import into mitochondria. The presequence is typically $\sim 20-60$ amino acid long, with a net positive charge. Presequences with $<10$ or $>65$ amino acids have also been reported. In this study, Atp17 displays the shortest presequence of 6 amino acids (Vögtle et al., 2009). Presequences are present in the form of an amphipathic $\alpha$-helix, with positively charged amino acids on one face of the helix and hydrophobic ones on the other (Schatz and Butow, 1983) (Allison and Schatz, 1986) (Roise et al., 1986). The different faces of the helix are recognised by specific receptors in the TOM, TIM23 and PAM complexes. Tom22 has a preference for the positive side of the presequence, whereas Tom20 binds to the hydrophobic side. The overall positive charge of the presequence is significant for its membrane potential $(\Delta \psi)$ dependent transport across the inner membrane (Martin et al., 1991). Recently, the mature portion of the protein was also shown to affect its dependence on the $\Delta \psi$ (Schendzielorz et al., 2017).

A presequence-containing protein can be destined for: (i) matrix, (ii) inner membrane and (iii) IMS. After import into the matrix, the presequence is usually recognised and cleaved by the mitochondrial processing peptidase (MPP) (Pfanner et al., 1988) (Schneider et al., 1998). An arginine at the C-terminus of the presequence acts as the MPP cleavage site (Taylor et al., 2001). An intermediate cleaving peptidase, Icp55, was also reported to remove a single amino acid from unstable MPP-cleaved intermediate proteins to promote their stability (Vögtle et al., 2009). Proteins targeted for the inner membrane or IMS 
contain a 16-18 amino acid 'sorting' signal after the MTS (Heijne et al., 1989) (Gakh et al., 2002), which arrests the protein in the TIM23 complex after which it is laterally released into the inner membrane. Proteins can either be anchored in the inner membrane via this signal or the signal can be cleaved off by proteases in the IMS, releasing the protein into the IMS (Glick et al., 1992) (Botelho et al., 2011).

Not all targeting sequences are at the $\mathrm{N}$-terminus. Proteins with unusual cleavable sequences have also been reported: (i) Bcs1, an IM protein, was found to have a positively charged sequence at the C-terminus of its transmembrane domain (Fölsch et al., 1996), (ii) Another IM protein, Hmi1, was reported to have a cleavable signal sequence at its C-terminus (Lee et al., 1999), (iii) For IM protein Pam18, no presequence could be identified in the precursor (Truscott et al., 2003), (iv) Multispanning inner membrane proteins can also utilise internal signals to insert themselves into the inner membrane via the TIM23 complex, as in the case of Sym1 (Reinhold et al., 2012) and (v) the hydrophobic extension at the $\mathrm{C}$-terminus of Mgr2 was also shown to act as a targeting signal (leva et al., 2013).

\subsubsection{The TIM23 complex}

The translocase of the inner mitochondrial membrane (TIM23 complex) is the primary import machinery for the majority of mitochondrial proteins destined for the matrix or inner membrane. Depending on the protein being imported, the TIM23 complex can exist in different forms (Figure 5). The core TIM23 complex (TIM23 ${ }^{\mathrm{CORE}}$ ) is made up of the channel-forming protein Tim23, the multispanning protein Tim17, the receptor Tim50 and potentially Mgr2 (Table 3).

Tim23 is an essential protein with four transmembrane domains and an IMS domain at the N-terminus (Emtage and Jensen, 1993) (Dekker et al., 1993). Through reconstitution and electrophysiology studies, it was shown that Tim23 forms a voltage-sensitive cationselective channel of $\sim 13-24 \AA$. Hence, precursor proteins need to be unfolded prior to translocation (Lohret et al., 1997) (Truscott et al., 2001). In addition, conserved amino acids lining the pore of the channel contribute to its cation selectivity (Denkert and Schendzielorz et al., 2017). Tim23 is also sensitive to presequences. Changes in the membrane potential or binding of presequence can cause structural changes in Tim23 and its interaction with other TIM23 complex subunits (Alder et al., 2008) (Malhotra et al., 2013) (Lytovchenko et al., 2013). 

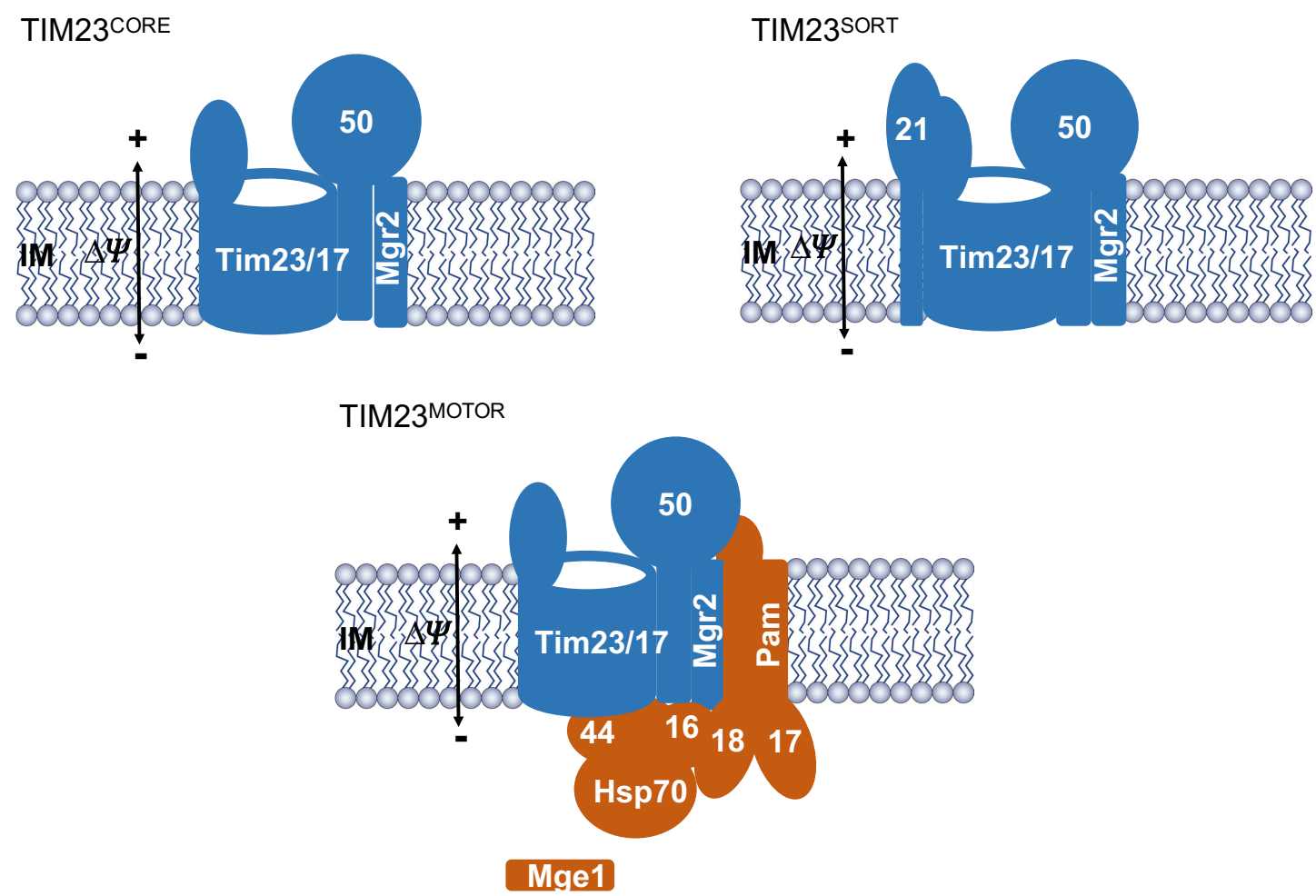

Figure 5 Different forms of the TIM23 complex in yeast - Top left: The polytopic channelforming proteins Tim23 and Tim17, the receptor protein Tim50 and the gatekeeper protein Mgr2 form the TIM23 ${ }^{\mathrm{CORE}}$; Top right: the membrane protein Tim21 associates with the CORE to form the TIM23 ${ }^{\text {SORT }}$ and links it to the respiratory complexes for lateral sorting of inner membrane proteins; Bottom: The PAM complex associates with TIM23 ${ }^{\text {CORE }}$ to form the TIM23 ${ }^{\text {MOTOR }}$ complex for import of matrix targeted proteins.

The Tim23 IMS domain is involved in interacting with presequences and other proteins of the TIM23 complex through their IMS domains. Additionally, the IMS domain has been reported to be intrinsically disordered (Gevorkyan-Airapetov et al., 2009) (la Cruz et al., 2010). The first half of the IMS domain (residues 1-50) has been suggested to span the outer membrane to potentially position the TIM23 complex in proximity to the TOM complex (Donzeau et al., 2000) to facilitate preprotein transfer. It was also shown that this segment responds to the import of precursor proteins via the TIM23 complex (PopovČeleketić et al., 2008). Through cross-linking studies, the second half of the Tim23 IMS domain (residues 51-100) has been shown to interact with a second Tim23 molecule. This dimerisation is proposed to occur via formation of leucine zippers between the two IMS domains, and is dependent on membrane potential. In the absence of membrane potential or the presence of presequences, the dimer becomes destabilised (Bauer et al., 1996) (Alder et al., 2008). Furthermore, this domain interacts with $\operatorname{Tim} 21^{\mathrm{IMS}}$, Tim50 ${ }^{\mathrm{IMS}}$ and Tom22 $2^{\text {IMS }}$ (Gevorkyan-Airapetov et al., 2009) (la Cruz et al., 2010) (Bajaj et al., 2014a). Therefore, Tim23 ${ }^{\mathrm{IMS}}$ has multiple sites for several interactions. However, the lack of evolutionary conservation of the extreme $\mathrm{N}$-terminal segment (residues 1-50) in higher eukaryotes along with it being dispensable for yeast growth suggests that this domain is 
not essential for protein import (Chacinska et al., 2005) (Bauer et al., 1996). Taken together, these studies reveal a flexible conformation of the Tim23 IMS domain acting as a platform for multiple interactions.

Table 3 Components of the TIM23 complex. Subunits of the TIM23 complex with their known function.

\begin{tabular}{|l|l|}
\hline Protein & Function \\
\hline Tim23 & Channel-forming subunit \\
\hline Tim50 & IMS presequence receptor, Tim23-Tim17 channel gating \\
\hline Tim17 & Contributes to Tim23 channel formation and regulation \\
\hline Tim21 & $\begin{array}{l}\text { Involved in TIM23-TOM and TIM23-respiratory chain } \\
\text { interaction }\end{array}$ \\
\hline Mgr2 & Lateral gatekeeper, couples Tim21 to Tim23-Tim17 \\
\hline
\end{tabular}

Tim17 is an essential integral membrane protein and a core component of the TIM23 complex (Ryan et al., 1994) (Maarse et al., 1994). Structurally, it is similar to Tim23, but it lacks an IMS domain (Kübrich et al., 1994). The four transmembrane helices traverse the inner membrane and are important for the structural integrity of the TIM23 complex. Although purified Tim23 can form a channel by itself, Tim17 is required for regulating the pore and voltage gating in vivo (Martinez-Caballero et al., 2007). Negatively charged residues in the N-terminal IMS, as well as a highly conserved disulfide bond in the IMS are critical for preprotein import and structural integrity of the complex (Ramesh et al., 2016) (Wrobel et al., 2016). Cross-linking studies suggest that TM1 of Tim23 is in proximity to TM4 of Tim17 under physiological conditions, and changes in membrane potential or presequence causes conformational changes in Tim23. The C-terminal IMS domain of Tim23 is also in proximity to Tim50 IMS (Alder et al., 2008). TM2 of Tim23 was also demonstrated to undergo voltage dependent conformational rearrangement (Alder et al., 2008) (van der Laan et al., 2013). Mutations in the GxxxG motifs of TM1 and TM2 of Tim23 affect its interactions with Tim17 and overall structural integrity of the complex (Demishtein-Zohary et al., 2015).

The inner membrane maintains a strongly impermeable barrier, which is also true for the Tim23 channel. Having it in a permanently open state would be futile. An essential protein involved in this regulation is Tim50, which is a single transmembrane spanning protein with a large hydrophilic domain in the IMS (Geissler et al., 2002) (Yamamoto et al., 2002). 
Truncated versions of Tim50 indicate that the IMS domain by itself is sufficient for its function (Mokranjac et al., 2009). This domain is in proximity to the C-terminus of Tim23 ${ }^{\text {IMS }}$ (Yamamoto et al., 2002) (Alder et al., 2008) and was found to be involved in Tim23 channel gating. In the absence of a preprotein, the channel is closed and Tim23 is in its oligomerised state. In the presence of a precursor, the channel becomes open to translocate proteins (Meinecke et al., 2006). In this way, the membrane potential across the inner membrane can be maintained. The IMS domain of Tim50 also binds to incoming precursors and facilitates their transfer to the TIM23 complex channel. Therefore, Tim50 acts as a receptor for proteins destined for the matrix or being sorted into the IM. Residues 395-476 at the C-terminus of Tim50 are involved in presequence binding and are termed PBD (presequence binding domain) (Schulz et al., 2011). Also, a second presequence binding site is present in the Tim50 core (Lytovchenko et al., 2013). Crystal structure of conserved residues 164-361 (IMS core) pointed to the presence of a negatively charged groove, which could bind to the presequence (Qian et al., 2011). Furthermore, NMR studies of shortened PBD (residues 400-450) demonstrated that this region is important for presequence binding (Rahman et al., 2014). Therefore, Tim50 acts as the central presequence receptor for the TIM23 complex.

Recently, another integral membrane component of the TIM23 complex, called Mgr2, was identified. It spans the IM twice, with a matrix loop between the two TM domains (Gebert et al., 2012) (leva et al., 2013). Mgr2 has been implicated in binding hydrophobic sorting signals and controlling the release of these signal-containing proteins into the IM. This process is also termed lateral release (leva et al., 2014). Deletion of Mgr2 shows an increased sorting of proteins, therefore it is also known as a lateral gatekeeper. Mgr2 also regulates the recruitment of newly imported Pam18 to the TIM23 ${ }^{\text {MOTOR }}$ complex (Schulz and Rehling, 2014).

Tim21 is an integral membrane protein with a single transmembrane domain (Chacinska et al., 2005). The IMS-exposed C-terminal domain is the main platform for interacting with Tim50 and Tom22 ${ }^{\mathrm{IMS}}$. Tim21 is a non-essential component of the TIM23 complex which helps in its association with the TOM complex and the respiratory chain complexes (Chacinska et al., 2005) (Albrecht et al., 2006) (van der Laan et al., 2006). The TIM23 complex, isolated via a tag on Tim21, could be reconstituted into cardiolipin-containing proteoliposomes. This complex showed similar channel activity as recombinant Tim23 and could also integrate membrane protein into the liposome (van der Laan et al., 2007). Additionally, the structure of Tim21 ${ }^{\mathrm{MS}}$ (residue 103-225) has been solved using crystallography (Albrecht et al., 2006). Furthermore, it was shown to interact with Tom22 $2^{\mathrm{IMS}}$ via NMR studies. The surface exposed residues of Tim2 $1^{\mathrm{IMS}}$ have a net charge 
of +8 , and were found to comprise of positively charged, negatively charged, hydrophobic or amphipathic residues. Predominant among these were the positively charged surface areas. This was shown to be the site for electrostatic interactions with Tom22 ${ }^{\mathrm{IMS}}$, which has a net charge of -5 . Mutation and cross-linking analysis showed that charged amino acids between residues 131-147 of Tom22 support its interactions with Tim21. Therefore, Tim21 is also involved in linking the TOM complex to the TIM23 complex, specifically with the transfer of proteins from Tom22 to the TIM23 complex. Tim21 is also required for sorting of membrane proteins into the inner membrane, as will be discussed at the end of the next section.

\subsubsection{The Presequence translocase associated motor (PAM) complex}

Complete import of a protein into the matrix requires two driving forces: membrane potential across the IM and ATP. The presequence translocase associated motor (PAM) complex utilises this second driving force. The PAM complex is composed of the tethering protein Tim44, the ATP driven chaperone Hsp70, the J-protein Pam18, the J-like protein Pam16, the nucleotide exchange factor Mge1 and Pam17 (Table 4 and Figure 6).

Table 4 Components of the PAM complex. Subunits of the PAM complex with their known function.

\begin{tabular}{|l|l|}
\hline Protein & Function \\
\hline Tim44 & Couples mtHsp70 to TIM23, binds to presequences \\
\hline Pam16 & J-like protein, recruits and controls Pam18 \\
\hline Pam17 & $\begin{array}{l}\text { Imports motor subunit, interacts with Tim23 } \\
\text { activity }\end{array}$ \\
\hline Pam18 & $\begin{array}{l}\text { Hsp70 family ATPase, involved in protein translocation and } \\
\text { folding }\end{array}$ \\
\hline $\begin{array}{l}\text { mtHsp70 } \\
\text { (Ssc1) }\end{array}$ & Nucleotide release factor for mtHsp70 \\
\hline Mge1 & \\
\hline
\end{tabular}

The molecular chaperones from the Hsp70 family are involved in a variety of functions including protein folding, disassembly and translocation across membranes. The mitochondrial Hsp70 family ATPase (mtHsp70), also known as Ssc1, can be present as a membrane-associated form, or as a soluble form, with protein translocation and folding functions respectively (Kang et al., 1990) (Horst et al., 1997). It is an essential component of the PAM complex and uses energy from ATP hydrolysis to drive protein translocation across the TIM23 complex. Like other Hsp70 family members, mtHsp70 has two 
prominent domains, the N-terminal nucleotide binding domain (NBD) and the C-terminal substrate binding domain (SBD). These two domains are connected by an interdomain linker. Furthermore, the SBD is made up of a substrate binding cleft and a lid (Craig, 2018). The mechanism of Hsp70-substrate interaction is well established (Takeda and McKay, 1996) (Mapa et al., 2010) (Mayer, 2013). In the presence of ATP, mtHsp70 binds the substrate in a low affinity state, such that the substrate on-off rate is high. This is the 'open' position. Due to low intrinsic ATPase activity, mtHsp70 requires an external Jprotein to facilitate ATP hydrolysis. In the presence of a J-protein, ATP hydrolysis takes place. The conformational changes lead to the formation of a 'closed' state, in which the lid closes over the cleft. Substrate interaction is thus stabilised in the ADP-bound form. A new cycle is initiated by ADP to ATP exchange by a nucleotide exchange factor (Hartl and Hayer-Hartl, 2002).

Structural studies of bacterial Hsp70 (DnaK) and its corresponding J-protein (DnaJ), as well as mammalian Hsp70, indicate that the conserved J-domain of J-proteins is involved in the interaction with Hsp70 (Wall et al., 1994) (Szyperski et al., 1994). Specifically, helix 2, helix 3 and the conserved HPD motif in the loop between them interact with the interdomain linker and proximal residues of Hsp70. The altered interdomain linker conformation affects the NBD-SBD interaction, stimulating ATP hydrolysis in the NBD (Greene et al., 1998) (Bukau and Horwich, 1998) (Jiang et al., 2005) (Jiang et al., 2007) (Swain et al., 2007).

In mitochondria, other key players of the Ssc1 cycle are Pam18, Mdj1 and Mge1. Pam18 is the essential inner membrane J-protein co-chaperone of Ssc1 at the motor complex, and Mdj1 acts as the J-protein for the Ssc1 soluble form (Truscott et al., 2003) (D'Silva et al., 2003) (Rowley et al., 1994). Pam18 spans the IM once, with its J-domain containing C-terminus present in the matrix. Mge1 is the essential soluble nucleotide exchange factor for Ssc1 (Laloraya et al., 1994) (Schneider et al., 1996) and is required by different mtHsp70 proteins (Ssc1 and Ssq1) (Schmidt et al., 2001). Recently it was shown that Mge1 does not facilitate the release of ADP, but rather the binding of ATP (Sikor et al., 2013). 


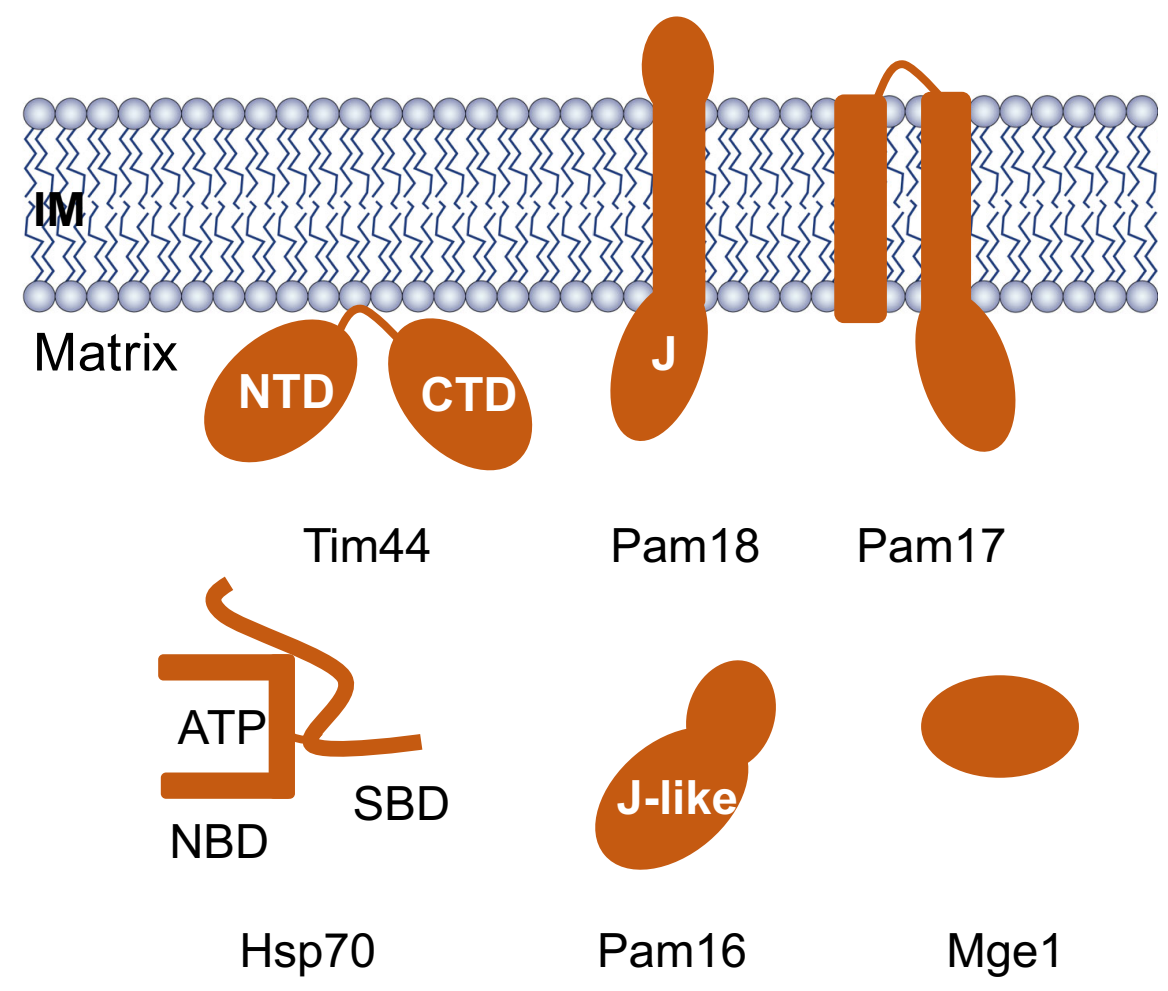

Figure 6 Components of the PAM complex - The PAM complex constitutes of the chaperone protein Hsp70, the tethering protein Tim44, the J-protein Pam18, the J-like protein Pam16, the nucleotide exchange factor Mge1 and the IM protein Pam17. Figure modified from (Craig, 2018).

Two other essential components of the PAM complex are Tim44 and Pam16. Tim44 is a peripheral IM-associated protein on the matrix side, which attaches to the membrane through interactions of its C-terminal helices (Weiss et al., 1999) (Marom et al., 2009). Tim44 couples mtHsp70 to the TIM23 complex and directs incoming preproteins towards mtHsp70 (Blom et al., 1993) (Kronidou et al., 1994) (Schneider et al., 1994). Furthermore, full length Tim44 has been shown to interact with presequences (Marom et al., 2011). Tim44 consists of two domains, the N-terminal domain (NTD, residues 43 to 209) and the C-terminal domain (CTD, residues 210 to 431). The NTD was recently shown to be intrinsically disordered and to contain residues required for interaction with presequences (Ting et al., 2017). Apart from binding to presequences, the NTD has also been shown to bind to Hsp70, Pam16 and Tim23 (Schiller et al., 2008) (Schilke et al., 2012) (Ting et al., 2014) (Ting et al., 2017). Hsp70 interaction sites are scattered throughout the NTD, as both NBD and SBD of Hsp70 interact with Tim44 (Krimmer et al., 2000) (Moro et al., 2002) (D'Silva et al., 2004). For Pam16, the N-terminus was reported to bind to Tim44 (Schilke et al., 2012). For the CTD, amino acids 234-425 were reported to form an $\alpha$ helix$\beta$ barrel domain (Josyula et al., 2006). Two $\mathrm{N}$-terminal helices of the CTD were reported to associate with the membrane (Marom et al., 2009). The CTD also interacts with Tim23 and Tim17 (Craig, 2018). Both the NTD and the CTD of Tim44 interact with the matrix- 
facing loop 1 of Tim23 (Ting et al., 2017). This loop is present between TM1 and TM2 of Tim23. However, exactly how a single loop of 24 residues binds to two separate domains of Tim44 is unknown. Loop 3 of Tim17, between TM3 and TM4, also interacts with Tim44 (Demishtein-Zohary et al., 2017). Therefore, interactions between Tim44 and matrix loops of core TIM23 complex subunits leads to stabilisation of Tim44 at the translocase, where it can also act as a docking platform for other motor components. Although the two domains have been shown to rescue Tim44 function when expressed separately, cell growth was found to be poor, pointing towards underlying complexities of its mechanism of action (Banerjee et al., 2015).

Pam16, another essential component of the motor, is a co-chaperone of mtHsp70 (Frazier et al., 2004) (Kozany et al., 2004). It is tethered at the IM through interactions with Tim44. Pam16 is known to be a J-like protein, since it has a matrix domain that is sequentially similar to the J-domain, but lacks the HPD motif, which is required to stimulate the ATPase activity of Hsp70. In fact, Pam16 was shown to repress the activity of Pam18 (Li et al., 2004), and structural analysis of the Pam16-Pam18 heterodimer revealed that the Pam18 HPD motif was occupied by Pam16 (Mokranjac et al., 2006). Pam16 and Pam18 are present in a 1:1 stoichiometry in mitochondria, since separately, both were shown to be unstable proteins, whereas the heterodimer is relatively more stable (losefson et al., 2007). The heterodimer associates with the TIM23 complex via multiple interactions. Apart from the N-terminal interaction of Pam16 with Tim44, the Pam18 N-terminus interacts with Tim17 in the IMS (Chacinska et al., 2005) (D'Silva et al., 2008) and Pam16 interacts with the Pam18 HPD motif. Together, these interactions regulate the position of different motor components with respect to each other and the TIM23 complex.

The latest PAM complex protein to be discovered is Pam17 (van der Laan et al., 2005). It has two TM domains connected by an IMS loop and a small domain in the matrix, which has been shown to interact with Tim17 loop 1 (Ting et al., 2014). The role of Pam17 is not very well defined. It has been suggested to help in the co-operation between TIM23 and PAM for protein import, based on its interaction with the Tim23-Tim17 core (PopovČeleketić et al., 2008). Tim44 inactivation results in increased association of Pam17 to TIM23 (Hutu et al., 2008). Therefore, Pam17 tends to associate with the TIM23 complex under specific physiological conditions. Recently, it was also found that Tim50 is required for recruiting Pam17 to TIM23 and that the import of specific $\Delta \psi$-hypersensitive proteins is aided by Pam17 (Schendzielorz et al., 2017).

To summarise, the TIM23 complex can exist in different states depending on the nature of the precursor protein being imported (Figure 5). The PAM complex and Tim21 are in a 
state of competition to associate with the TIM23 ${ }^{\text {CORE }}$ complex. Association of Tim21 with TIM23 ${ }^{\text {CORE }}$ leads to formation of the TIM $23^{\text {SORT }}$, which is predominantly required for lateral sorting of proteins into the inner membrane and can also associate with the respiratory chain (van der Laan et al., 2007). TIM23 ${ }^{\text {CORE }}$-PAM together form the TIM23 ${ }^{\text {MOTOR }}$ complex, which is utilised by matrix targeted proteins. Varying the levels of Tim21 or PAM subunits leads to a shift between these forms (Popov-Čeleketić et al., 2008). Upon inactivation of Pam16, the TIM23 complex shifts to the TIM23 ${ }^{\text {SORT }}$ form. Overexpression of Tim21 also leads to less PAM complex association with TIM23 ${ }^{\mathrm{CORE}}$ (Chacinska et al., 2010). For a long time, TIM23 $3^{\text {SORT }}$ and TIM23 $3^{\text {MOTOR }}$ were thought to operate individually for the import of sorted and matrix proteins, respectively. However, studies have shown that TIM23 SORT and TIM23 $3^{\text {MOTOR }}$ are in a state of dynamic exchange during the import of precursor proteins (Chacinska et al., 2010). TIM23-accumulated sorted and matrix proteins have been reported to interact with respiratory chain complexes (Wiedemann et al., 2007). Pam16 and Pam18 also interact with these complexes independent of Tim21. Therefore, the import of different precursor proteins is not exclusive to different forms of the TIM23 complex, but involves dynamic exchange and interaction of different subunits with the TIM23 ${ }^{\text {CORE }}$ complex.

\subsubsection{The translocation intermediate: TOM-TIM23 supercomplex}

The question regarding the transport of mitochondrial proteins across the two membranes remained unresolved for a long time: was the transfer occurring in two separate steps, or was it a single event occurring via fusion of membrane components? Early electron microscopy analysis of rat liver mitochondria revealed the presence of contact sites between the inner and outer mitochondrial membrane, which were proposed to play a role in the passage of solutes between the cytosol and the matrix (Hackenbrock, 1968). These were later defined as translocation contact sites (Schleyer and Neupert, 1985).

A precursor could be accumulated in the translocation intermediate such that the $\mathrm{N}$ terminus was processed and the C-terminus was susceptible to added proteases. Therefore, the accumulated protein could span both the membranes simultaneously. Such an intermediate can be formed by: (i) importing the protein at low temperature (Schleyer and Neupert, 1985), (ii) binding the protein with antibodies against the C-terminus before import (Schleyer and Neupert, 1985) (Schwaiger et al., 1987), (iii) import at low levels of ATP (Pfanner et al., 1987c) and (iv) import of fusion proteins with an N-terminal MTS and a stably folded domain at the C-terminus (Rassow et al., 1989) (Figure 7). Following the discovery of a stable translocation contact site for the import of proteins, it was suggested that the essential components of these sites could be distinct proteins in both membranes, which could provide a hydrophilic environment for the precursor protein to pass through 
(Pfanner et al., 1987a). The $\mathrm{b}_{2}(167)$-DHFR fusion protein, which consisted of $\mathrm{N}$-terminal 167 residues of cytochrome $b_{2}$ fused to mouse DHFR (dihydrofolate reductase), could be accumulated at the translocation contact sites due to the tight folding of the DHFR moiety in the presence of methotrexate (Rassow et al., 1989). Electron microscopy with this protein accumulated in mitochondria revealed that the two membranes were not fused and the bilayers were separated by 18-20 nm (Rassow et al., 1989).

As various components of the translocation machinery were discovered, the preprotein spanning both membranes was found to be associated with both the TIM23 and the TOM complex (Dekker et al., 1997). The TOM complexes are more abundant in mitochondria than the TIM23 complexes and as such, only one in four TOM complexes was associating with TIM23 for transporting the precursor (Dekker et al., 1997). Preprotein import sites in mitochondria were also found to be distributed non-uniformly over the mitochondrial surface. The active TOM-TIM23 supercomplexes were revealed to be in the vicinity of cristae junctions (Gold et al., 2014).

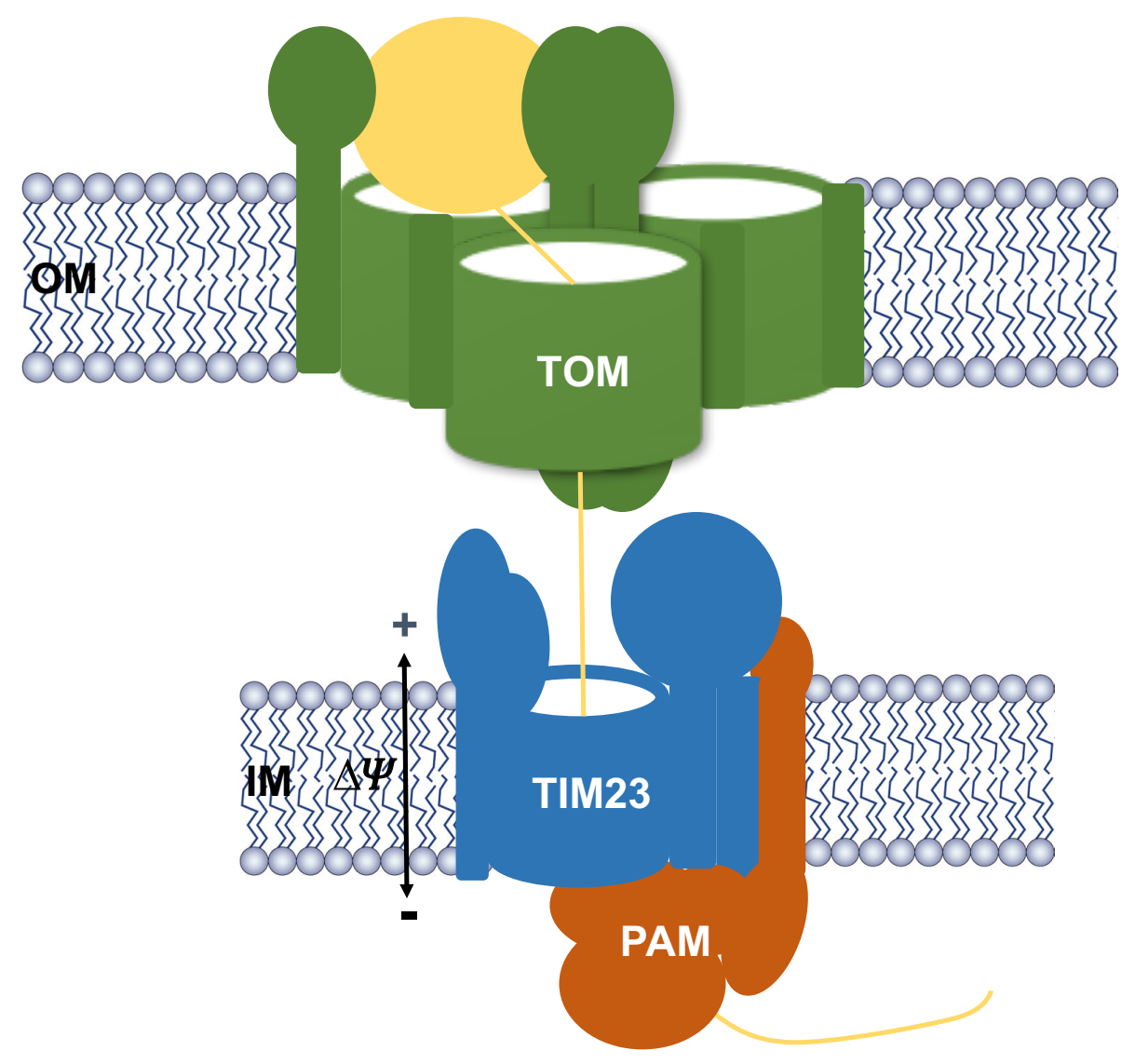

Figure 7 The TOM-TIM23 supercomplex formation - TOM and TIM23 complexes are in proximity to each other during import of a precursor protein. One of the ways this complex can be generated in vivo or in organello is by arresting the import of a preprotein with a tightly folded domain at its C-terminus. 
As the molecular mechanisms for different components of the TOM, TIM23 and PAM complexes emerged, the transfer of proteins from the TOM to the TIM23 complex became clearer. Tom $22^{\mathrm{IMS}}$ is required for stabilisation and Tim50 for the generation of the TOMTIM23 intermediate (Chacinska et al., 2003). The IMS domains of both Tom22 and Tim50 act as presequence receptors, and Tim23 can also recognise presequences. Tim2 $1^{\mathrm{IMS}}$ has been shown to bind to Tom22 ${ }^{\mathrm{IMS}}$ (Chacinska et al., 2005) (Albrecht et al., 2006).

Therefore, a strong interplay between the IMS domains of Tom22, Tim23, Tim21 and Tim50 is involved in the transfer of proteins from the TOM to the TIM23 complex. At the trans side of the TOM complex, the presequence of a precursor protein is recognised by Tom $22^{\mathrm{IMS}}$ (Shiota et al., 2011). Subsequently, the binding of Tim $21^{\mathrm{IMS}}$ to Tom $22^{\mathrm{IMS}}$ leads to dissociation of the precursor protein from Tom22 (Chacinska et al., 2005). The released presequence then binds to Tim50. Tim50 and Tim21 IMS domains can interact with each other with high affinity, and this interaction can be affected by Tim23 ${ }^{\text {IMS }}$ (Lytovchenko et al., 2013). The Tim50-Tim23 interaction causes the presequence to be released from Tim50, following which it can be handed over to the Tim23 channel. Until now, different forms of the TOM-TIM23 supercomplex have been reported, either associated with PAM or Tim21 (Chacinska et al., 2010), but these proteins are more in dynamic exchange during the import of protein and less likely to be found as stable constituents of the supercomplex. Furthermore, various constituents of the TOM-TIM23 supercomplex are in a state of being recruited and exchanged to promote import motor activity (Schulz and Rehling, 2014). Therefore, it appears that the complex mechanism of protein transfer, along with the interplay of interactions between the TOM, TIM23 and PAM complex subunits during active import of a protein, is not fully elucidated.

\subsection{Aims of the work}

The translocases of the inner mitochondrial membrane, the TIM23 and the TIM22 complexes, are crucial for the import of presequence-containing and hydrophobic signalcontaining proteins respectively. Together, these complexes are responsible for the import of majority of inner membrane and matrix proteins of mitochondria. Therefore, insights into the structure and substrate spectrum of these complexes is important to understand their underlying mechanism of action.

The role of various TIM23 complex components has been defined over the last few decades. However, structural information of the TIM23 complex is essential to put the puzzle together with respect to the various transient and dynamic interactions occurring within its subunits. With that in mind, the aim of the first project was to isolate the TIM23 
complex and the TOM-TIM23 supercomplex in preparatory amounts to analyse it with single particle cryo-electron microscopy. In addition, this data was to be complemented with a cross-linking mass spectrometry approach to identify protein interactions and spatial organisation of proteins within the complex.

The second part of this study is dedicated to identifying and broadening our knowledge about the proteins which depend on the TIM22 complex for their import. A very limited number of proteins are currently known to import via the TIM22 complex, due to which their targeting information is not fully understood. These include both four and six transmembrane domain-containing proteins. Therefore, identification of new substrates would provide information regarding the targeting signals utilised by the TIM22 complex for import of these proteins. 


\section{Materials and Methods}

\subsection{Materials}

\subsubsection{Chemicals, kits and buffers}

\subsubsection{Chemicals}

Standard chemicals used in this study were obtained from AppliChem (Darmstadt, Germany), BD (Heidelberg, Germany), Roche (Mannheim, Germany), Merck, Novagen and Calbiochem (Darmstadt, Germany), Roth (Karlsruhe, Germany), Serva (Heidelberg, Germany) and Sigma-Aldrich (Taufkirchen, Germany). The complete list of individual reagents can be found in Table 5 .

Table 5 List of chemicals used in this study and their suppliers.

\begin{tabular}{|l|l|}
\hline Chemical & Supplier \\
\hline$\left[{ }^{35}\right.$ S]-L-methionine & Hartmann Analytic \\
\hline 2-mercaptoethanol & Sigma-Aldrich \\
\hline 6-aminocaproic acid & Sigma-Aldrich \\
\hline Acetic acid & Roth \\
\hline Acetone & Roth \\
\hline Acrylamide/bisacrylamide $(37.5: 1)$ solution & Roth \\
\hline Acrylamide, 4x crystallised & Roth \\
\hline Agarose NEEO ultra-quality & Roth \\
\hline Ammonium persulfate & Roth \\
\hline Ampicillin & AppliChem \\
\hline Antimycin & Sigma-Aldrich \\
\hline ATP & Roche \\
\hline Bacto Agar & BD \\
\hline Bacto Peptone & BD \\
\hline BactoTryptone & BD \\
\hline
\end{tabular}




\begin{tabular}{|c|c|}
\hline Bacto Yeast Extract & BD \\
\hline Bio-Rad Protein Assay & Bio-Rad \\
\hline Bis-Acrylamide & Roth \\
\hline Bis-Tris Buffer grade & AppliChem \\
\hline Bovine serum albumin & Sigma-Aldrich \\
\hline Bromophenol Blue & Merck \\
\hline BS2G & Thermo Scientific \\
\hline BS3 & Thermo Scientific \\
\hline Complete EDTA-free protease inhibitor mix & Roche \\
\hline Coomassie Brilliant Blue G-250 & Roth \\
\hline Coomassie Brilliant Blue R-250 & Roth \\
\hline Creatine kinase & Roche \\
\hline Creatine phosphate & Roche \\
\hline CSM-amino acid & MP Biomedicals \\
\hline D-Desthiobiotin & IBA \\
\hline DMSO & AppliChem \\
\hline DNA ladder mix “Gene Ruler” & Thermo Scientific \\
\hline DSG & Thermo Scientific \\
\hline DSS & Thermo Scientific \\
\hline DTT & Roth \\
\hline EDTA & Roth \\
\hline Ethanol & Roth \\
\hline Ethidium bromide $0.025 \% \mathrm{w} / \mathrm{v}$ & Roth \\
\hline Galactose, $\mathrm{D}(+)$ & Roth \\
\hline Glucose, $\mathrm{D}(+)$ & Roth \\
\hline GDN101 & Anatrace \\
\hline L-Glutathione reduced & Sigma Aldrich \\
\hline Glutaraldehyde EM Grade & Polysciences \\
\hline Glycerol & Sigma-Aldrich \\
\hline Glycine & Roth \\
\hline HEPES & Roth \\
\hline Herring sperm DNA & Promega \\
\hline Hydrochloric acid $37 \% \mathrm{w} / \mathrm{v}$ & Roth \\
\hline
\end{tabular}


Materials and Methods

\begin{tabular}{|c|c|}
\hline Imidazole & Sigma Aldrich \\
\hline IPTG & Sigma Aldrich \\
\hline KanamycinSulfate & Sigma Aldrich \\
\hline Lithium acetate & AppliChem \\
\hline LMNG & Anatrace \\
\hline Magnesium chloride heptahydrate & Merck \\
\hline Methanol & Roth \\
\hline Methionine & Roth \\
\hline MitoTracker Orange CMTMRos & Thermo Scientific \\
\hline MOPS & Sigma-Aldrich \\
\hline $\mathrm{NADH}$ & Roche \\
\hline $\mathrm{Ni}^{2+}$-NTA agarose & Macherey-Nagel \\
\hline Oligomycin & Sigma-Aldrich \\
\hline Ortho-phosphoric acid & Merck \\
\hline PEG-4000 (polyethylene glycol 4000) & Merck \\
\hline PMSF & Roth \\
\hline Potassium chloride & Roth \\
\hline Potassium dihydrogen phosphate & Roth \\
\hline Potassium hydrogen diphosphate & Roth \\
\hline Proteinase $\mathrm{K}$ & Roche \\
\hline Restriction Enzymes & Thermo Scientific \\
\hline Roti-Quant & Roth \\
\hline SDS & Roth \\
\hline SDS-PAGE protein standard & Serva \\
\hline Lipodisq SMA Copolymer 3:1 & Sigma-Aldrich \\
\hline Sodium chloride & Roth \\
\hline Sodium hydroxide & AppliChem \\
\hline Sorbitol & Roth \\
\hline Strep-tactin Sepharose $50 \%$ suspension & IBA \\
\hline Sucrose & Roth \\
\hline TCA & Merck \\
\hline TEMED & Roth \\
\hline Tricine & Roth \\
\hline
\end{tabular}




\begin{tabular}{|l|l|}
\hline Tris & Roth \\
\hline Tween-20 & Roth \\
\hline Urea & Roth \\
\hline Valinomycin & Sigma-Aldrich \\
\hline Yeast nitrogen base without amino acids & BD \\
\hline Zymolase 20 T & Seikagaku Biobusiness Corporation \\
\hline
\end{tabular}

\subsubsection{Kits}

Commercial kits used in this study are listed in Table 6. They were used according to the instructions from the manufacturer.

Table 6 List of commercial kits used in this study along with their manufacturer.

\begin{tabular}{|l|l|}
\hline Kit & Supplier \\
\hline Alkaline phosphatase, shrimp & Roche Applied Science \\
\hline $\begin{array}{l}\text { ECL Plus Western Blotting Detection } \\
\text { Reagent }\end{array}$ & Thermo Scientific \\
\hline FastDigest restriction enzymes & Thermo Scientific \\
\hline $\begin{array}{l}\text { Flexi Rabbit Reticulocyte Lysate } \\
\text { System }\end{array}$ & Promega \\
\hline Immobilon-P Transfer membrane & Millipore \\
\hline KOD Hot Start DNA Polymerase & Merck \\
\hline $\begin{array}{l}\text { mMessagemMachine SP6 } \\
\text { transcription } \\
\text { Kit }\end{array}$ & Invitrogen \\
\hline Rapid DNA Ligation Kit & Thermo Scientific \\
\hline $\begin{array}{l}\text { TNT Quick Coupled Transcription/ } \\
\text { Translation SP6 }\end{array}$ & Promega \\
\hline Wizard SV Gel and PCR Clean-Up & Promega \\
\hline Wizard SV Mini-Prep & Promega \\
\hline
\end{tabular}

\subsubsection{Buffer and Media Recipes}

Recipes of the buffers and culture media used is provided in Table 7. All solutions were prepared using analytical grade chemicals. Media for bacteria and yeast growth was autoclaved post preparation.

Table 7 List of commonly used buffers in this study along with their composition.

\begin{tabular}{|l|l|}
\hline \hline Buffer & Components \\
\hline Amino acid-free media & $0.67 \%(w / v)$ yeast nitrogen base w/o amino acids, $2 \%$ \\
\hline
\end{tabular}




\begin{tabular}{|c|c|}
\hline & sugar, $0.07 \%$ CSM-amino acid \\
\hline Ampicillin & $100 \mathrm{mg} / \mathrm{ml}$, sterile filtered \\
\hline AVO mix & $\begin{array}{l}1 \mathrm{mM} \text { antimycin, } 0.1 \mathrm{mM} \text { valinomycin, } 2 \mathrm{mM} \\
\text { oligomycin in ethanol }\end{array}$ \\
\hline Bacteria Lysis Buffer & $\begin{array}{l}40 \mathrm{mM} \text { Tris } / \mathrm{HCl}, \mathrm{pH} 7.4,500 \mathrm{mM} \mathrm{NaCl}, 10 \mathrm{mM} \\
\text { Imidazole, } 1 \mathrm{mg} / \mathrm{ml} \text { lysozyme, } 0.5 \mathrm{mM} \text { PMSF and } \\
\text { Complete EDTA-free protease inhibitor mix }\end{array}$ \\
\hline BN anode buffer & $50 \mathrm{mM}$ Bis-Tris $\mathrm{pH} 7.0$ \\
\hline BN cathode buffer & $\begin{array}{l}50 \mathrm{mM} \text { tricine, } 15 \mathrm{mM} \text { Bis-Tris, with or without } 0.02 \% \\
\text { Coomassie Brilliant Blue G-250 }\end{array}$ \\
\hline BN gel buffer & $67 \mathrm{mM}$ 6-aminocaproic acid, 50 mM Bis-Tris pH 7.0 \\
\hline BN sample loading buffer & $\begin{array}{l}0.5 \% \text { (w/v) Coomassie Brilliant Blue G-250, } 50 \text { mM 6- } \\
\text { aminocaproic acid, } 10 \text { mM Bis-Tris pH 7.0 }\end{array}$ \\
\hline Carrier DNA & Herring sperm DNA $(10 \mathrm{mg} / \mathrm{ml})$ in TE buffer \\
\hline $\begin{array}{l}\text { Colloidal Coomassie staining } \\
\text { solution }\end{array}$ & $\begin{array}{l}0.1 \%(\mathrm{w} / \mathrm{v}) \text { Coomassie Brilliant Blue G-250, } 2 \%(\mathrm{w} / \mathrm{v}) \\
\text { phosphoric acid, 10\% (w/v) ammonium sulfate, } 20 \% \\
\text { methanol }\end{array}$ \\
\hline Coomassie staining solution & $\begin{array}{l}1.5 \mathrm{~g} / \mathrm{l} \text { Coomassie Brilliant Blue R-250, } 40 \% \text { ethanol, } \\
10 \% \text { acetic acid }\end{array}$ \\
\hline Destaining solution & $30 \%$ ethanol, $10 \%$ acetic acid \\
\hline Detergent solubilisation buffer & $\begin{array}{l}20 \mathrm{mM} \text { HEPES/KOH pH 7.4, } 150 \mathrm{mM} \mathrm{NaCl}, 10 \% \\
\text { glycerol, } 0.1 \mathrm{mM} \text { EDTA, } 1 \% \text { detergent }\end{array}$ \\
\hline DTT buffer & 10 mM DTT, 100 mM Tris/HCl pH 9.4 \\
\hline EDTA & 0.5 M EDTA, pH 8.0 \\
\hline Glutaraldehyde & $25 \%$ solution, EM grade \\
\hline $10 \%$ Glycerol buffer & $\begin{array}{l}10 \% \text { glycerol, } 20 \mathrm{mM} \text { HEPES/KOH, } \mathrm{pH} 7.4,150 \mathrm{mM} \\
\mathrm{NaCl}, 0.1 \mathrm{mM} \text { EDTA, detergent as indicated }\end{array}$ \\
\hline $30 \%$ Glycerol buffer & $\begin{array}{l}30 \% \text { glycerol, } 20 \mathrm{mM} \text { HEPES/KOH, } \mathrm{pH} 7.4,150 \mathrm{mM} \\
\mathrm{NaCl}, 0.1 \mathrm{mM} \text { EDTA, detergent as indicated, } \\
\text { glutaraldehyde as indicated }\end{array}$ \\
\hline Homogenisation buffer & $\begin{array}{l}0.6 \mathrm{M} \text { sorbitol, } 10 \mathrm{mM} \text { Tris/HCl } \mathrm{pH} 7.4,1 \mathrm{mM} \text { EDTA, } \\
0.2 \%(\mathrm{w} / \mathrm{v}) \text { fatty acid free BSA, } 1 \mathrm{mM} \text { PMSF }\end{array}$ \\
\hline Import buffer & $\begin{array}{l}3 \%(\mathrm{w} / \mathrm{v}) \text { fatty acid free bovine serum albumin, } 250 \\
\mathrm{mM} \text { sucrose, } 80 \mathrm{mM} \mathrm{KCl}, 5 \mathrm{mM} \mathrm{MgCl}_{2}, 10 \mathrm{mM} \text { MOPS- } \\
\mathrm{KOH} \mathrm{pH} 7.2,5 \mathrm{mM} \text { methionine, } 2 \mathrm{mM} \mathrm{KH}_{2} \mathrm{PO}_{4}, 2 \mathrm{mM} \\
\text { ATP, } 2 \mathrm{mM} \text { NADH, with or without } 100 \mu \mathrm{g} / \mathrm{ml} \text { creatine } \\
\text { kinase (CK) and } 5 \mathrm{mM} \text { creatine phosphate (CP) }\end{array}$ \\
\hline LB medium & $\begin{array}{l}1 \%(\mathrm{w} / \mathrm{v}) \text { tryptone, } 0.5 \%(\mathrm{w} / \mathrm{v}) \mathrm{NaCl}, 1 \%(\mathrm{w} / \mathrm{v}) \text { yeast } \\
\text { extract }\end{array}$ \\
\hline LB Agar & $\begin{array}{l}\text { LB medium with } 1.5 \%(\mathrm{v} / \mathrm{w}) \text { agar, sterilised by } \\
\text { autoclaving }\left(20 \mathrm{~min}, 120^{\circ} \mathrm{C}\right)\end{array}$ \\
\hline Lithium acetate/PEG & $\begin{array}{l}0.1 \mathrm{M} \text { lithium acetate, } 40 \% \text { polyethylene glycol } 4000 \text { in } \\
\text { water, filter sterilised }\end{array}$ \\
\hline Methotrexate & $\begin{array}{l}10 \mathrm{mM} \text { stock in water, used as } 2000 \mathrm{X}, \text { stored frozen } \\
\text { at }-20^{\circ} \mathrm{C} \text { in single-use aliquots }\end{array}$ \\
\hline PMSF stock & $0.2 \mathrm{M}$ PMSF in ethanol \\
\hline
\end{tabular}




\begin{tabular}{|c|c|}
\hline $\begin{array}{l}\text { Potassium phosphate buffer } \\
\text { pH } 7.4\end{array}$ & $80.2 \%(w / v) \mathrm{K}_{2} \mathrm{HPO}_{4}, 19.8 \%(w / v) \mathrm{KH}_{2} \mathrm{PO}_{4}$ \\
\hline Resolving gel (for SDS-PAGE) & $\begin{array}{l}\text { 10-16\% acrylamide, } 80 \mathrm{mM} \text { Tris- } \mathrm{HCl} \mathrm{pH} 6.8,0.1 \% \\
\text { SDS, } 0.1 \% \text { APS, } 0.05 \% \text { TEMED }\end{array}$ \\
\hline SDS Protein loading dye (PLD) & $\begin{array}{l}10 \% \text { glycerol, } 2 \%(w / v) \text { SDS, } 0.01 \% \text { bromophenol } \\
\text { blue, } 60 \mathrm{mM} \text { Tris/HCl pH } 6.8\end{array}$ \\
\hline SDS running buffer & $25 \mathrm{mM}$ Tris, $192 \mathrm{mM}$ glycine, $0.1 \%$ (w/v) SDS \\
\hline SEM & $\begin{array}{l}250 \mathrm{mM} \text { sucrose, } 10 \mathrm{mM} \text { MOPS-KOH } \mathrm{pH} 7.2,1 \mathrm{mM} \\
\text { EDTA }\end{array}$ \\
\hline SMA solubilisation buffer & $\begin{array}{l}50 \mathrm{mM} \text { HEPES/KOH pH 7.4, } 150 \mathrm{mM} \mathrm{NaCl}, 5 \mathrm{mM} \\
\text { Imidazole, } 0.2 \mathrm{mM} \text { PMSF, } 20 \mathrm{mg} \mathrm{SMA} / \mathrm{ml} \text { of buffer }\end{array}$ \\
\hline SMA wash buffer & $\begin{array}{l}50 \mathrm{mM} \text { HEPES/KOH pH 7.4, } 150 \mathrm{mM} \mathrm{NaCl}, 5 \mathrm{mM} \\
\text { Imidazole }\end{array}$ \\
\hline Stacking gel (for SDS-PAGE) & $\begin{array}{l}4 \%(\mathrm{w} / \mathrm{v}) \text { arylamide, } 0.1 \%(\mathrm{w} / \mathrm{v}) \text { SDS, } 380 \mathrm{mM} \text { Tris- } \\
\mathrm{HCl} \mathrm{pH} \mathrm{8.8,0.1 \% (w/v)} \mathrm{APS,} \mathrm{0.05 \%} \mathrm{TEMED}\end{array}$ \\
\hline TAE buffer & $40 \mathrm{mM}$ Tris/acetate $\mathrm{pH}$ 8.0, 2 mM EDTA \\
\hline TBST & $50 \mathrm{mM}$ Tris, $150 \mathrm{mM} \mathrm{NaCl}, 0.05 \%$ Tween-20 \\
\hline TBS & $50 \mathrm{mM}$ Tris, $150 \mathrm{mM} \mathrm{NaCl}$ \\
\hline TCA solution & $72 \%$ trichloracetic acid in water \\
\hline Transfer Buffer & 25 mM Tris, 192 mM glycine, $10 \%$ ethanol \\
\hline Wash buffer & $\begin{array}{l}20 \mathrm{mM} \text { HEPES/KOH pH 7.4, } 150 \mathrm{mM} \mathrm{NaCl}, 10 \% \\
\text { glycerol, } 1 \mathrm{mM} \text { PMSF, } 0.1 \mathrm{mM} \text { EDTA, detergent as } \\
\text { indicated }\end{array}$ \\
\hline YPAD (2x) & $\begin{array}{l}2 \%(\mathrm{w} / \mathrm{v}) \text { yeast extract, } 4 \%(\mathrm{w} / \mathrm{v}) \text { peptone, } 4 \%(\mathrm{w} / \mathrm{v}) \\
\text { glucose, } 0.008 \%(\mathrm{w} / \mathrm{v}) \text { adenine hemisulfate, } \\
\text { autoclaved }\end{array}$ \\
\hline YPD & $\begin{array}{l}1 \% \text { yeast extract, } 2 \% \text { peptone, } 2 \% \text { glucose, } \\
\text { autoclaved }\end{array}$ \\
\hline YPG & $\begin{array}{l}1 \%(\mathrm{w} / \mathrm{v}) \text { yeast extract, } 2 \%(\mathrm{w} / \mathrm{v}) \text { peptone, } 3 \%(\mathrm{w} / \mathrm{v}) \\
\text { glycerol, autoclaved }\end{array}$ \\
\hline Zymolyase buffer & $1.2 \mathrm{M}$ sorbitol, $20 \mathrm{mM}$ potassium phosphate $\mathrm{pH} 7.4$ \\
\hline
\end{tabular}

\subsubsection{Antibodies}

Primary polyclonal antibodies were utilised for detection of $S$. cerevisiae proteins. The serum obtained after injecting an antigen (peptide or purified protein) into rabbits was diluted in TBS-T. Secondary goat anti-rabbit immunoglobulin antibodies were used as HRP conjugates $(1: 10,000)$. For detection of GFP-tagged proteins, the corresponding mouse monoclonal antibody (Roche) (1:1000) and HRP conjugated goat anti-mouse immunoglobulin antibody $(1: 10,000)$ were used. 


\subsubsection{E. coli strains and Plasmids}

E. coli strains used in this study are listed in Table 8. All plasmids used in this study were propagated in E. coli XL1 Blue cells. Table 9 lists all the plasmids utilised in this study.

\subsubsection{E. coli strains}

Table 8 E. coli strains used in this study along with their genotype.

\begin{tabular}{|c|c|c|}
\hline $\begin{array}{l}\text { E. coli } \\
\text { strain }\end{array}$ & Genotype & Reference \\
\hline XL1 Blue & $\begin{array}{l}\text { recA1 endA1 gyrA96 thi-1 hsdR17 } \\
\text { supE44 relA1 lac [F'proAB } \\
\left.\left.\text { lacl }{ }^{9} Z \Delta M 15 \text { Tn10(Tet }{ }^{\prime}\right)\right]\end{array}$ & Stratagene \\
\hline BL21 (DE3) & $\begin{array}{l}\text { fhuA2 [lon] ompT gal } \\
d c m h s d S \lambda(\mathrm{DE} 3)\left(\mathrm{r}_{\mathrm{B}}^{-} \mathrm{m}_{\mathrm{B}}^{-}\right) \mathrm{F}^{-}\end{array}$ & Stratagene \\
\hline
\end{tabular}

\subsubsection{Plasmids}

Table 9 List of plasmids used in this study.

\begin{tabular}{|l|l|l|l|l||}
\hline Plasmid & Backbone & Insert & Purpose & Reference \\
\hline $\begin{array}{l}\text { pRG6 } \\
\text { (AAC2-GFP) }\end{array}$ & pUG35 & AAC2-GFP & $\begin{array}{l}\text { AAC2-GFP } \\
\text { expression in } \\
\text { yeast }\end{array}$ & This study \\
\hline $\begin{array}{l}\text { pRG3 } \\
\text { (GFP-Mir1) }\end{array}$ & pUG36 & GFP-Mir1 & $\begin{array}{l}\text { GFP-Mir1 } \\
\text { expression in } \\
\text { yeast }\end{array}$ & This study \\
\hline $\begin{array}{l}\text { GST-nGFP- } \\
\text { Strep }\end{array}$ & pGEX-4T2 & $\begin{array}{l}\text { GST- } \\
\text { GFP } \\
\text { Nanobody- } \\
\text { Strep }\end{array}$ & $\begin{array}{l}\text { For expression } \\
\text { and purification } \\
\text { of GFP } \\
\text { Nanobody }\end{array}$ & $\begin{array}{l}\text { Provided by Dr. } \\
\text { Markus Deckers } \\
\text { (AG Rehling) }\end{array}$ \\
\hline $\begin{array}{l}\text { pCS153 } \\
\text { (SCGP) }\end{array}$ & K27 Sumo & $\begin{array}{l}\text { F1ß-MSP- } \\
\text { sfGFP }\end{array}$ & $\begin{array}{l}\text { SCGP } \\
\text { expression and } \\
\text { purification }\end{array}$ & $\begin{array}{l}\text { Provided by Dr. } \\
\text { Christian Schulz } \\
\text { (AG Rehling) }\end{array}$ \\
\hline $\begin{array}{l}\text { pRG9 } \\
\text { (Jac1-sfGFP) }\end{array}$ & K27 Sumo & $\begin{array}{l}\text { Jac1- } \\
\text { sfGFP }\end{array}$ & $\begin{array}{l}\text { Jac1-sfGFP } \\
\text { expression and } \\
\text { purification }\end{array}$ & This study \\
\hline $\begin{array}{l}\text { pRG10 } \\
\text { (Mam33- } \\
\text { sfGFP) }\end{array}$ & K27 Sumo & $\begin{array}{l}\text { Mam33- } \\
\text { sfGFP }\end{array}$ & $\begin{array}{l}\text { Mam33-sfGFP } \\
\text { expression and } \\
\text { purification }\end{array}$ & This study \\
\hline $\begin{array}{l}\text { pCS189 } \\
\text { (SCGP) }\end{array}$ & p425Gal1 & SCGP & $\begin{array}{l}\text { SCGP } \\
\text { expression in } \\
\text { yeast }\end{array}$ & $\begin{array}{l}\text { Provided by Dr. } \\
\text { (AG Rehling) }\end{array}$ \\
\hline $\begin{array}{l}\text { pRG13 } \\
\text { (Jac1-sfGFP) }\end{array}$ & p425Gal1 & $\begin{array}{l}\text { Jac1- } \\
\text { sfGFP }\end{array}$ & $\begin{array}{l}\text { Jac1-sfGFP } \\
\text { expression in } \\
\text { yeast }\end{array}$ & This study \\
\hline
\end{tabular}




\subsubsection{Yeast strains}

All yeast strains used in this study are derivatives of YPH499 and BY4741 wild type strains and are listed in Table 10.

Table 10 S. cerevisiae strains used in this study along with their genotype.

\begin{tabular}{|c|c|c|}
\hline Strain & Genotype & Reference \\
\hline YPH499 & $\begin{array}{l}\text { MATa ade2-101, his3- } \Delta 200 \text {, leu2- } \Delta 1 \text {, ura3- } \\
52, \text { trp1- } \Delta 63, \text { lys2-801 }\end{array}$ & (Sikorski et al., 1989) \\
\hline BY4741 & MATa his $3 \Delta 1$, leu $2 \Delta 0$, met $15 \Delta 0$, ura $3 \Delta 0$ & Euroscarf \\
\hline $\begin{array}{l}\text { His- } \\
\text { SUMOstar- } \\
\text { Tim23 } \\
\end{array}$ & $\begin{array}{l}\text { His-SUMOstar-Tim23 pRS413, tim23 } \\
\text { MATa ade2-101, his3- } \Delta 200 \text {, leu2- } \Delta 1 \text {, ura3- } \\
52 \text {, trp1- } \Delta 63 \text {, lys2-801, tim23::LYS2 }\end{array}$ & $\begin{array}{l}\text { Provided by Dr. Alex } \\
\text { Schendzielorz } \\
\text { (AG Rehling) }\end{array}$ \\
\hline $\begin{array}{l}\text { His-Nedd8- } \\
\text { Tim23 }\end{array}$ & $\begin{array}{l}\text { His-brNedd8-Tim23 in tim23 } \\
\text { MATa ade2-101, his3- } \Delta 200 \text {, leu2- } \Delta 1 \text {, ura3- } \\
52 \text {, trp1- } \Delta 63 \text {, lys2-801, tim23::LYS2 }\end{array}$ & $\begin{array}{l}\text { Provided by Dr. Alex } \\
\text { Schendzielorz } \\
\text { (AG Rehling) }\end{array}$ \\
\hline $\operatorname{tim} 22-14$ & $\begin{array}{l}\text { YPH499 22-M4 } \\
\text { MATa ade2-101, his3- } \Delta 200 \text {, leu2- } \Delta 1 \text {, ura3- } \\
52 \text {, trp1- } \Delta 63 \text {, lys2-801, tim22-M4 }\end{array}$ & (Wagner et al., 2008) \\
\hline $\operatorname{tim} 22-F 1$ & $\begin{array}{l}\text { YPH499 22-F1 } \\
\text { MATa ade2-101, his3- } \Delta 200 \text {, leu2- } \Delta 1 \text {, ura3- } \\
\text { 52, trp1- } \Delta 63 \text {, lys2-801, tim22-F1 }\end{array}$ & $\begin{array}{l}\text { Yeast collection } \\
\text { AG Rehling }\end{array}$ \\
\hline
\end{tabular}

\subsubsection{Instruments and softwares}

\subsubsection{Instruments}

Laboratory equipments used in this study are listed in Table 11.

Table 11 List of laboratory equipments used in this study along with their manufacturer.

\begin{tabular}{||l|l||}
\hline \hline Instrument & Manufacturer \\
\hline 5415 R (centrifuge) & Eppendorf \\
\hline 5424 (centrifuge) & Eppendorf \\
\hline 5804 R (centrifuge) & Eppendorf \\
\hline ÄKTA purifier & GE Healthcare \\
\hline Amersham Typhoon Phosphorlmager & GE Healthcare \\
\hline Autoradiography Storage Phosphor Screen & GE Healthcare \\
\hline Cawomat 2000 IR Developing machine & Agfa \\
\hline Delta Vision Fluorescence Microscope & Applied Precision \\
\hline
\end{tabular}




\begin{tabular}{|c|c|}
\hline EPS 601 power supply & GE Healthcare \\
\hline EmulsiFlex-C3 homogeniser & Avestin \\
\hline Fluorescence scanner FLA-9000 & Fujifilm \\
\hline GD-5040 Gel Dryers & Scie-Plas \\
\hline GeneAmp PCR System 9700 (thermo cycler) & Applied Biosystems \\
\hline Gradient Master & BioComp Instruments \\
\hline HiLoad 16/60 Superdex 200 & GE Healthcare \\
\hline HisTrap HP, $1 \mathrm{ml}$ & GE Healthcare \\
\hline Hoefer SE600 Ruby Blue native system & GE Healthcare \\
\hline JA-20 (rotor) & Beckman Coulter \\
\hline Magnetic Stirrer MR 3001 & Heidolph \\
\hline Mini-PROTEAN 3 Cell & Bio-Rad \\
\hline Mini-Sub Cell GT Cell & Bio-Rad \\
\hline NanoVueTM Spectrophotometer & GE Healthcare \\
\hline New Brunswick Innova 2300 & Eppendorf \\
\hline New Brunswick Innova 44 Shaker & Eppendorf \\
\hline Optima L-90 K Ultracentrifuge & Beckman Coulter \\
\hline Potter S (Dounce homogeniser) & Sartorius \\
\hline PowerPac HC Power Supply & Bio-Rad \\
\hline Semi Dry Blotting chamber & PEQLAB Biotechnologie \\
\hline Sorvall RC 6 Plus (centrifuge) & Thermo Scientific \\
\hline Sorvall RC 12BP (centrifuge) & Thermo Scientific \\
\hline Sorvall SS-34 (rotor) & Thermo Scientific \\
\hline Sorvall F10S-6x500Y (rotor) & Thermo Scientific \\
\hline Sorvall H-12000 (rotor) & Thermo Scientific \\
\hline SW $60 \mathrm{Ti}$ (rotor) & Beckman Coulter \\
\hline Thermomixer $\mathrm{C}$ & Eppendorf \\
\hline Varian Cary 50 UV-Vis spectrophotometer & Agilent Technologies \\
\hline Vortex-Genie 2 & Scientific Industries \\
\hline
\end{tabular}

\subsubsection{Softwares}

All softwares used for image visualisation and processing, quantification, data analysis, bioinformatics and structure visualisation are listed in Table 12. 
Table 12 List of softwares used in this study.

\begin{tabular}{|l|l||}
\hline Software & Producer \\
\hline DataGraph 4.3 & Visual Data Tools, Inc. \\
\hline Geneious 9.1.2 & Biomatters, Auckland, New Zealand \\
\hline Fiji Image Processing & $\begin{array}{l}\text { Johannes Schindelin, Albert Cardona, Mark Longair, } \\
\text { Benjamin Schmid, and others }\end{array}$ \\
\hline Illustrator CS6 & Adobe Systems, San Jose, CA, USA \\
\hline ImageQuant TL & GE Healthcare BioSciences AB, Uppsala, Sweden \\
\hline Microsoft Office & Microsoft Corporation, Redmond, WA, USA \\
\hline Papers 2.8.1 & Mekentosj, Aalsmeer, Netherlands \\
\hline Photoshop CS6 & Adobe Systems, San Jose, CA, USA \\
\hline PyMol & Schrödinger, Portland, OR, USA \\
\hline $\begin{array}{l}\text { Softworx Image } \\
\text { Acquisition Software }\end{array}$ & Applied Precision, Bratislava, Slovakia \\
\hline
\end{tabular}

\subsection{Methods}

\subsubsection{Handling of E. coli}

\subsubsection{E. coli growth}

E. coli strains BL21 and XL1 Blue were cultured in LB media or LB Agar plate according to standard procedures (Sambrook and Russell, 2001). For antibiotic resistance, $100 \mu \mathrm{g} / \mathrm{ml}$ ampicillin or $50 \mu \mathrm{g} / \mathrm{ml}$ kanamycin was added to the media. Optical density at $600 \mathrm{~nm}$ $\left(\mathrm{OD}_{600}\right)$ was used to monitor cell growth. Cryo stocks were generated by mixing $800 \mu \mathrm{l}$ of a logarithmic phase culture with $200 \mu \mathrm{l}$ of sterile glycerol.

\subsubsection{E. coli transformation}

Competent E. coli cells were prepared using $\mathrm{CaCl}_{2}$ method (Sambrook and Russell, 2001). Briefly, E. coli cells were inoculated from LB agar plate to LB media. After overnight growth with vigorous shaking at $37^{\circ} \mathrm{C}$, the cells were diluted $1: 100$ in LB media and grown till $0.6 \mathrm{OD}_{600}$. After keeping them on ice for $5 \mathrm{~min}$, the cells were harvested by centrifugation at $2000 \times \mathrm{g}$ for $10 \mathrm{~min}$ and resuspended in $250 \mathrm{ml}$ cold sterile $50 \mathrm{mM}$ $\mathrm{CaCl}_{2}$ solution. After $15 \mathrm{~min}$ on ice, the cells were harvested followed by resuspension in $25 \mathrm{ml} 50 \mathrm{mM} \mathrm{CaCl}_{2} .15 \%$ glycerol was added for storage of cells. $100 \mu \mathrm{l}$ aliquots were prepared and stored at $-80^{\circ} \mathrm{C}$.

For transformation, the $100 \mu \mathrm{l}$ aliquot was thawed on ice. 100-200 ng plasmid DNA or 10 $\mu \mathrm{l}$ ligation mix was added. Gentle mixing was done, followed by incubation on ice for 15 
min. Cells were transferred to $42^{\circ} \mathrm{C}, 30 \mathrm{~s}$ for BL21 and $45 \mathrm{~s}$ for $\mathrm{XL} 1$ Blue. After this, the cells were cooled down on ice for $2 \mathrm{~min}$. $900 \mu \mathrm{l}$ of LB media was added, and the cells were incubated at $37^{\circ} \mathrm{C}$ with vigorous shaking for $1 \mathrm{~h}$. Cells were harvested, resuspended in $100 \mu$ LB media and plated on LB agar plate with appropriate selection marker.

\subsubsection{Handling of S. cerevisiae}

\subsubsection{Yeast growth}

Media used in this study for cultivating S. cerevisiae include YPD, YPG, 2X YPAD, as well as selective amino acid-free media such as SD-Ura (selective glucose without Uracil) and SSuc-Leu (selective sucrose without Leucine) for strains that required additional selection. $2 \%$ sugar or $3 \%$ glycerol was used as a carbon source. All liquid cultures were grown at $30^{\circ} \mathrm{C}$ with shaking (160-220 rpm), except for temperature sensitive strains which were cultivated at $24^{\circ} \mathrm{C}$. Heat stress to ts strains was provided at $37^{\circ} \mathrm{C}$. For growth on plates, $1.5 \%$ agar was added to the corresponding media. For comparing growth of different strains, serial dilutions of an overnight growing culture were prepared and plated on appropriate plates. These plates were incubated at $24^{\circ} \mathrm{C}, 30^{\circ} \mathrm{C}$ and $37^{\circ} \mathrm{C}$ for $3-5$ days. Glycerol cryo stocks were prepared by mixing yeast cells from a plate with $1 \mathrm{ml}$ YPAD with $15 \%$ glycerol and freezing it at $-80^{\circ} \mathrm{C}$.

\subsubsection{Transformation in yeast}

Competent yeast cells were prepared using a modified Lithium acetate (LiAc)/PEG method (Gietz and Woods, 2002). Briefly, yeast strains were pre-cultured in 2X YPAD overnight. Next day, the culture was diluted to $\mathrm{OD}_{600}$ of about 0.2 in $2 X$ YPAD, and grown for 3-4 $\mathrm{h}$ at the corresponding growth temperature of that strain. Cells were harvested at $\mathrm{OD}_{600}$ 0.6-0.8 by centrifugation for $5 \mathrm{~min}$ at $3000 \times \mathrm{g}$. After washing once with sterile water followed by washing with $0.1 \mathrm{M} \mathrm{LiAc}$, the cells were resuspended in $2 \mathrm{ml} 0.1 \mathrm{M} \mathrm{LiAc}$ and centrifuged at $12,000 \times \mathrm{g}$ for $30 \mathrm{~s}$ at $4^{\circ} \mathrm{C}$. The pellet was resuspended in $2 \mathrm{ml} 0.1 \mathrm{M} \mathrm{LiAc}$ and $100 \mu \mathrm{l}$ aliquots were prepared. Cells were stored at $-80^{\circ} \mathrm{C}$. For transformation, $120 \mu \mathrm{g}$ herring sperm DNA was added to $100 \mu \mathrm{l}$ of thawed competent cells. Following this, $300 \mathrm{ng}$ of plasmid DNA or $2 \mu \mathrm{g}$ of purified PCR product was added. Cells were incubated for 30 min at appropriate temperature with mild agitation, after which $600 \mu \mathrm{l}$ of LiAc/PEG solution was added. After this, cells were incubated for 60-90 $\mathrm{min}$ at appropriate temperature under shaking. $68 \mu \mathrm{l}$ DMSO was added and the cells were transferred to $42^{\circ} \mathrm{C}$ for $15 \mathrm{~min}$. Finally, the cells were harvested at $100 \times \mathrm{g}$ for $2 \mathrm{~min}$, followed by resuspension in $1 \mathrm{M}$ sorbitol and seeding on selection plates. 


\subsubsection{Whole cell lysate}

For analysis of protein expression in yeast cells, whole cell lysates were prepared (Yaffe and Schatz, 1984). Briefly, yeast cells were cultured in an appropriate medium and $3 \times 10^{7}$ cells $\left(3 \mathrm{OD}_{600}\right)$ were harvested at $3000 \times \mathrm{g}$ for $5 \mathrm{~min}$. Following this, the cells were resuspended in $1 \mathrm{ml}$ water, followed by addition of $148 \mu \mathrm{l} 2 \mathrm{M} \mathrm{NaOH}$ and $12 \mu \mathrm{l} \beta$ mercaptoethanol. After incubation on ice for $10 \mathrm{~min}, 160 \mu \mathrm{l} 50 \%$ TCA was added. This was followed by another incubation on ice for $10 \mathrm{~min}$. Samples were centrifuged at 14,000 $\mathrm{x} \mathrm{g}$ for $10 \mathrm{~min}$ at $4^{\circ} \mathrm{C}$. The pellet was resuspended in $50 \mu \mathrm{l}$ SDS PLD. Analysis was carried out by SDS-PAGE and western blotting.

\subsubsection{Preparation of mitochondria}

Differential centrifugation of yeast cells was done to obtain mitochondria from them (Meisinger et al., 2006). To start with, a pre-culture was prepared by inoculating yeast cells from a plate in $5 \mathrm{ml}$ YPD media. Subsequent pre-cultures were prepared in 50 and $300 \mathrm{ml}$ YPG. The last pre-culture was used to inoculate the final culture $(1.8 \mathrm{~L})$. Culture volumes were varied based on the amounts of mitochondria required. Cells were incubated overnight with shaking at $160 \mathrm{rpm}$ at $30^{\circ} \mathrm{C}\left(24^{\circ} \mathrm{C}\right.$ for ts strain and $37^{\circ} \mathrm{C}$ for heat shock of ts strains). The following day, cells with $0 \mathrm{D}_{600} 1.5-2.5$ were harvested by centrifugation at $4000 \times \mathrm{g}$ for $10 \mathrm{~min}$. The pellet was washed with water and then resuspended in DTT buffer (2 $\mathrm{ml}$ buffer/g cell pellet, see Table 7 ). They were incubated for $30 \mathrm{~min}$ at appropriate temperature with shaking. The cells were harvested by centrifugation at $3000 \times \mathrm{g}$ for $10 \mathrm{~min}$, washed with $1.2 \mathrm{M}$ Sorbitol and resuspended in Zymolase buffer $(7 \mathrm{ml}$ buffer/g cell pellet). $4 \mathrm{mg}$ Zymolase/g cell pellet was added to digest the cell wall, and the cells were incubated for $1 \mathrm{~h}$ with shaking at the appropriate temperature. After this, the cells were harvested and washed with zymolase buffer. All the subsequent steps were carried out at $4^{\circ} \mathrm{C}$. Cold homogenisation buffer was added to the pellet $(7 \mathrm{ml}$ buffer/g cell pellet) and the resuspended cells were homogenised for $15-20$ strokes at $700 \mathrm{rpm}$ using a $60 \mathrm{ml}$ homogeniser. The homogenate was centrifuged at 5000 $x \mathrm{~g}$ for $5 \mathrm{~min}$. The supernatant obtained was centrifuged for $10 \mathrm{~min}$ at $7000 \mathrm{xg}$. Finally, centrifugation of the supernatant obtained from the previous step was performed at $15,000 \times \mathrm{g}$ for $15 \mathrm{~min}$ to obtain the crude mitochondrial fraction. The resulting pellet was washed with $5 \mathrm{ml}$ SEM buffer containing $1 \mathrm{mM}$ PMSF, following which it was dissolved in an appropriate volume of SEM buffer. Protein concentration was determined by Bradford assay. Briefly, serial dilutions of IgG standard were prepared, at concentrations of $7.5,15$, 30 and $60 \mu \mathrm{g} / \mathrm{ml}$. For mitochondria, a 1:20 dilution was prepared in water. 5, 10 and $20 \mu \mathrm{l}$ of this dilution was used for protein estimation. $1 \mathrm{ml}$ of $1 \mathrm{x}$ Roti-Quant was added. Samples 
were incubated for $5 \mathrm{~min}$, following which absorption at $595 \mathrm{~nm}$ was measured. Final mitochondria concentration was adjusted to $10 \mathrm{mg} / \mathrm{ml}$ in SEM buffer. Prepared mitochondria were aliquoted in appropriate volume, flash frozen in liquid nitrogen and stored at $-80^{\circ} \mathrm{C}$.

For preparing mitochondria from the Tim22 temperature sensitive strain (tim22-14) and the corresponding WT, the yeast cells were cultured at $24^{\circ} \mathrm{C}$. After growing the cells till late log phase at $24^{\circ} \mathrm{C}$, they were shifted to $37^{\circ} \mathrm{C}$ for 15,25 and $40 \mathrm{~h}$. Subsequently, mitochondria were prepared as described above.

\subsubsection{Microscopy of yeast cells}

Yeast cells were grown in selective media (SD-Ura or SSuc-Leu) depending on the plasmid transformed. Cells transformed with SCGP and Jac1-sfGFP containing plasmids were inoculated in SSuc-Leu media and grown overnight at $30^{\circ} \mathrm{C}$ under shaking. Next day, galactose was added to final concentration of $2 \%$, for induction of protein expression. Cells were visualised at different time points using a DeltaVision fluorescence microscope. For this, $1 \mu$ MitoTracker was added to $2 \mathrm{ml}$ of culture. Cells were kept on shaking for 20 min. Afterwards, the cells were harvested at $3000 \times \mathrm{g}$ for 4 min and washed with SSucLeu. The pellet was resuspended in $200 \mu \mathrm{l}$ SSuc-Leu. $4.5 \mu \mathrm{l}$ of the suspension was applied on a glass slide, after which it was covered with a cover slip. Immersion oil was applied on top, and the cells were visualised under the Pol, FITC and TRITC channel.

STED microscopy of yeast cells expressing Jac1-sfGFP was performed by Dr. Stefan Stoldt (Research Group Mitochondrial Structure and Dynamics, Max Planck Institute for Biophysical Chemistry, Göttingen, Germany). Antibody against Tom40 was used as a marker for mitochondria.

WT and tim22-14 cells transformed with plasmids containing AAC2-GFP and GFP-Mir1 were grown in SD-Ura media overnight at $24^{\circ} \mathrm{C}$. Next day, MitoTracker treatment and visualisation was done as mentioned above.

\subsubsection{Molecular Biology Techniques}

\subsubsection{Plasmid isolation from $E$. coli}

$5 \mathrm{ml}$ E. coli culture was harvested by centrifugation at $12,000 \times \mathrm{g}$ for $5 \mathrm{~min}$. Wizard Plus SV Miniprep DNA Purification System (Promega) was used for isolating the DNA. Instructions from the manufacturer were followed. Briefly, the cell pellet was resuspended in $250 \mu \mathrm{l}$ resuspension buffer. $250 \mu \mathrm{l}$ lysis buffer was added along with $10 \mu \mathrm{l}$ alkaline protease. The sample was mixed by inverting 5 times and incubated at room temperature for 5 min. Afterwards, $350 \mu \mathrm{l}$ neutralisation buffer was added. Samples were centrifuged 
at $16,000 \times \mathrm{g}$ for $10 \mathrm{~min}$. The supernatant was applied on a filter column and centrifuged for $1 \mathrm{~min}$ at $16,000 \times \mathrm{g}$. Subsequently, the filter column was washed with 750 and $250 \mu \mathrm{l}$ wash buffer. After spinning the column dry, DNA was eluted by incubating the column with $50 \mu \mathrm{dH}_{2} \mathrm{O}$ for $1 \mathrm{~min}$ followed by centrifugation at $16,000 \times \mathrm{g}$ for $1 \mathrm{~min}$. Plasmid DNA concentration was estimated using NanoVue Spectrophotometer (GE Healthcare) and DNA was stored at $-20^{\circ} \mathrm{C}$.

\subsubsection{Yeast genomic DNA isolation}

For preparing genomic DNA from yeast, YPH499 cells were grown in YPD at $30^{\circ} \mathrm{C}$ overnight. On the next day, the cells were harvested at $3000 \times \mathrm{g}$ for $5 \mathrm{~min}$. They were resuspended in $150 \mu \mathrm{l}$ of $50 \mathrm{mM}$ Tris/ $\mathrm{HCl} \mathrm{pH} \mathrm{7.5,10} \mathrm{mM} \mathrm{EDTA,} \mathrm{0.3 \%} \beta$-mercaptoethanol and $0.25 \mathrm{mg} / \mathrm{ml}$ zymolase. After incubation at $37^{\circ} \mathrm{C}$ for $1 \mathrm{~h}, 20 \mu \mathrm{l}$ of $10 \%$ SDS and $100 \mu \mathrm{l}$ of $8 \mathrm{M}$ ammonium acetate was added. After mixing, the solution was incubated for $15 \mathrm{~min}$ at $-20^{\circ} \mathrm{C}$. Subsequently, centrifugation was carried out at $15,000 \times \mathrm{g} .180 \mu \mathrm{l}$ of the supernatant was mixed with equal volumes of isopropanol. The mixture was centrifuged at $15,000 \times \mathrm{g}$ for $15 \mathrm{~min}$ at $4^{\circ} \mathrm{C}$. The pellet obtained was washed with $70 \%$ ethanol, dried and resuspended in $100 \mu$ l water.

\subsubsection{Polymerase Chain Reaction (PCR)}

DNA segments were amplified (Saiki et al., 1985) from yeast genomic DNA and plasmid DNA for molecular cloning and transformation using KOD Hot Start DNA Polymerase (Merck). For each $50 \mu \mathrm{l}$ reaction, 10-100 ng of template was added. This was supplemented by $0.2 \mathrm{mM}$ dNTPs, $1.5 \mathrm{mM} \mathrm{MgSO}_{4}$, $1 \mathrm{X}$ KOD buffer, $0.4 \mu \mathrm{M}$ forward and reverse primers, $1 \cup$ KOD Hot Start Polymerase and 2\% DMSO. Cycle conditions were as follows: $95^{\circ} \mathrm{C}$ for $2 \mathrm{~min}$ for denaturation of template DNA, $30 \mathrm{~s}$ at $95^{\circ} \mathrm{C}, 10 \mathrm{~s}$ at $50-58^{\circ} \mathrm{C}$ (according to the primers used) and $1 \mathrm{~min}$ at $70^{\circ} \mathrm{C}$ for 30 cycles. PCR products were analysed by agarose gel electrophoresis (Scharf et al., 1986) combined with EtBr staining for DNA visualisation. Briefly, $1 \%(w / v)$ agarose solution was prepared in TAE buffer. Agarose was dissolved by heating the mixture. Ethidium bromide was added to a final concentration of $1 \mu \mathrm{g} / \mathrm{ml}$. The gel was allowed to polymerise at room temperature.

PCR products were purified using Wizard SV Gel and PCR Clean-Up System (Promega) according to the manufacturer's instructions. Briefly, PCR product was mixed with $150 \mu \mathrm{l}$ binding buffer and applied on a filter column. After incubation of $1 \mathrm{~min}$, it was centrifuged for $1 \mathrm{~min}$ at $16,000 \times \mathrm{g}$. Filter column was washed with 700 and $500 \mu \mathrm{l}$ wash buffer. Elution was carried out by incubating the dried column with $50 \mu \mathrm{dH}_{2} \mathrm{O}$ for 1 min followed 
by centrifugation at $16,000 \times \mathrm{g}$ for $1 \mathrm{~min}$. DNA concentration was estimated using NanoVue Spectrophotometer (GE Healthcare).

\subsubsection{Molecular Cloning}

Cloning was carried out using restriction enzymes according to established procedures (Sambrook and Russell, 2001). In short, both the plasmid and the PCR product were digested with appropriate FastDigest restriction enzymes (Thermo Scientific). $30 \mu \mathrm{l}$ reactions were prepared containing $1 \mu$ l of both restriction enzymes, $1 \mathrm{X}$ FastDigest buffer and 500-800 ng DNA. The reactions were incubated for $30 \mathrm{~min}$ at $37^{\circ} \mathrm{C}$. Digested fragments were purified as described in the previous section (2.2.3.3). For ligation, reaction mixture with $5 \mu$ ldigested plasmid, $10 \mu$ digested insert, $4 \mu$ DNA Ligation buffer and $1 \mu \mathrm{l}$ T4 DNA Ligase (Rapid DNA Ligation Kit, Thermo Scientific) was prepared. Following incubation at $22^{\circ} \mathrm{C}$ for $30 \mathrm{~min}, 10 \mu \mathrm{l}$ of the mixture was transformed into competent E. coli XL1 cells (see section 2.2.1.2). Clones obtained were analysed by restriction digestion and sequencing (SeqLab, Göttingen).

\subsubsection{5 in vitro transcription and translation}

mMessagemMachine SP6 transcription kit (Invitrogen) was used to generate mRNAs in vitro. Based on the manufacturer's instructions, a $20 \mu$ reaction mixture with $1 \mu \mathrm{g}$ PCR product, $2 \mu \mathrm{l}$ enzyme mix, $1 \mathrm{X}$ NTP/CAP and $1 \mathrm{X}$ reaction buffer was prepared. Transcription was carried out at $37^{\circ} \mathrm{C}$ for $90 \mathrm{~min}$. This was followed by a TURBO DNasel treatment for $15 \mathrm{~min}$ at $37^{\circ} \mathrm{C}$. To precipitate the mRNA, $30 \mu \mathrm{LiCl}$ solution was added, following which the sample was frozen at $-20^{\circ} \mathrm{C}$ for $30 \mathrm{~min}$. The precipitated mRNA was obtained by centrifuging the sample at $16,000 \times \mathrm{g}$ for $15 \mathrm{~min}$ at $4^{\circ} \mathrm{C}$. After washing the pellet with $70 \%$ ethanol, it was dried at room temperature. $30 \mu \mathrm{l}$ nuclease-free water was used to resuspend the pellet. mRNA concentration was estimated using NanoVue Spectrophotometer (GE Healthcare), after which it was stored at $-80^{\circ} \mathrm{C}$.

For synthesis of radiolabelled protein precursors, in vitro translation was carried out using either TNT Quick Coupled Transcription/Translation SP6 kit (Promega) for plasmids or Flexi Rabbit Reticulocyte Lysate System (Promega) for mRNA. Manufacturer's instructions were used to prepare the reaction. For translation from mRNA, $33 \mu \mathrm{l}$ Flexi Rabbit Reticulocyte Lysate, $1 \mu \mathrm{l} 1 \mathrm{mM}$ amino acid mix-Met, $1 \mu \mathrm{g}$ mRNA and $50 \mu \mathrm{Ci}{ }^{35} \mathrm{~S}$ Met were mixed. $\mathrm{KCl}$ and $\mathrm{MgAc}$ amounts were optimised depending on the precursor. The reaction was incubated at $30^{\circ} \mathrm{C}$ for $90 \mathrm{~min}$, following which it was quenched by $250 \mathrm{mM}$ sucrose. Prepared lysates were used directly for import reactions. 


\subsubsection{Protein analysis}

\subsubsection{Protein purification}

For expression of recombinant proteins in $E$. coli, plasmids were transformed into competent BL21 cells as described (see section 2.2.1.1). Cells were inoculated in a preculture of $50 \mathrm{ml}$ LB media with appropriate selection marker $(100 \mu \mathrm{g} / \mathrm{ml}$ ampicillin or 30 $\mu \mathrm{g} / \mathrm{ml}$ kanamycin) and grown overnight at $37^{\circ} \mathrm{C}$. The next day, it was diluted in the final culture $(2 \mathrm{~L})$ to an $\mathrm{OD}_{600}$ of 0.1 . Cells were grown till $\mathrm{OD}_{600}$ reached 0.6 , after which they were treated with $1 \mathrm{mM}$ IPTG for induction of protein expression. Cells were grown for $4 \mathrm{~h}$ with shaking (160 rpm) at $37^{\circ} \mathrm{C}$ for expression of SCGP, Jac1-sfGFP and Mam33-sfGFP, and at $30^{\circ} \mathrm{C}$ for expression of GFP nanobody. After expression, cells were harvested by centrifugation at $4000 \times \mathrm{g}$ for $10 \mathrm{~min}$. After a washing step with water, cell pellet was either used directly or frozen at $-20^{\circ} \mathrm{C}$ till needed. For opening the cells, the pellet was resuspended in bacteria lysis buffer (with additional components according to the protein being purified). The suspension was applied to Emulsiflex C3 homogeniser (Avestin) for 3 rounds of opening the cells. Following this, the suspension was centrifuged at $20,000 \times \mathrm{g}$ twice for $20 \mathrm{~min}$. The supernatant was filtered using $0.2 \mu \mathrm{m}$ pore size filters and applied to their respective affinity columns. For SCGP, Jac1-sfGFP and Mam33-sfGFP, which have a $6 \mathrm{X}$ His tag followed by Sumo protease cleavage site at the $\mathrm{N}$-terminus, HisTrap columns (GE Healthcare) were used. Purification procedure was the same for all three proteins and was carried out using ÄKTA Purifier 10 (GE Healthcare). Briefly, the filtered supernatant obtained after lysis was applied to a HisTrap column (column volume $1 \mathrm{ml}$ ) preequilibrated with 10 column volumes of HisTrap buffer A $(40 \mathrm{mM}$ Tris/ $\mathrm{HCl}$ pH 7.4, $500 \mathrm{mM}$ $\mathrm{NaCl}, 10 \mathrm{mM}$ Imidazole). The loading was done at a flow rate of $0.5 \mathrm{ml} / \mathrm{min}$. Following this, the column was washed with 20 column volumes of HisTrap buffer A and eluted with a linear gradient of HisTrap buffer $B(40 \mathrm{mM}$ Tris/HCl pH 7.4, $500 \mathrm{mM} \mathrm{NaCl}, 500 \mathrm{mM}$ Imidazole) at a flow rate of $1 \mathrm{ml} / \mathrm{min}$. Fractions were collected, and analysis was done using SDS-PAGE followed by Coomassie Brilliant Blue staining. Fractions containing protein of interest were pooled. Dialysis was carried out overnight using a SnakeSkin membrane (Thermo Scientific) in dialysis buffer $(20 \mathrm{mM}$ Tris/HCl pH 7.4, $100 \mathrm{mM} \mathrm{NaCl})$. After dialysis, the protein solution was concentrated with Amicon centrifugal filters (Merck) with a $10 \mathrm{kDa}$ cut-off column. SUMO protease was added ( $1 \mathrm{mg}$ protease/200 $\mathrm{mg}$ protein) along with $1 \mathrm{mM}$ DTT, and the sample was left overnight at $4^{\circ} \mathrm{C}$ on a shaker. Next day, size exclusion chromatography was performed using HiLoad 16/60 Superdex 200 columns (GE Healthcare). Columns were pre-equilibrated with 2 column volumes of corresponding buffer $(20 \mathrm{mM}$ Tris/ $\mathrm{HCl} \mathrm{pH} \mathrm{7.4,} 100 \mathrm{mM} \mathrm{NaCl})$. After this, protein was 
applied to the column at a flow-rate of $1 \mathrm{ml} / \mathrm{min}$. Fractions were collected to keep a track of protein separation and purification analysis was assessed using SDS PAGE and Coomassie Brilliant Blue staining. Desired fractions were pooled together. Proteins were aliquoted, snap-frozen and stored at $-20^{\circ} \mathrm{C}$.

For GFP nanobody, GSTrap column (GE Healthcare) was used. The affinity purification procedure was same as above, except for the buffers used (Buffer $\mathrm{A}: 140 \mathrm{mM} \mathrm{NaCl}, 2.7$ $\mathrm{mM} \mathrm{KCl}, 10 \mathrm{mM} \mathrm{Na} 2 \mathrm{HPO}_{4}, 1.8 \mathrm{mM} \mathrm{KH} \mathrm{PO}_{4}, \mathrm{pH}$ 7.3; buffer B: $50 \mathrm{mM}$ Tris/HCl, $10 \mathrm{mM}$ reduced glutathione, $\mathrm{pH}$ 8.0). After affinity purification, the protein was aliquoted and stored at $-20^{\circ} \mathrm{C}$.

\subsubsection{SDS-PAGE}

Separation of proteins was analysed by SDS polyacrylamide gel electrophoresis ((Laemmli, 1970) with modifications). Polyacrylamide gels of varying percentage were prepared with acrylamide:bisacrylamide ratio $37.5: 1$ containing $0.1 \%$ SDS. Stacking gel was composed of $4 \%$ acrylamide in Tris buffer $\mathrm{pH} 6.8$, whereas resolving gel contained $10-16 \%$ acrylamide in Tris buffer $\mathrm{pH}$ 8.8. Samples were prepared in SDS protein loading dye by incubating them at $95^{\circ} \mathrm{C}$ for $5 \mathrm{~min}$. The gels were run in SDS running buffer at 25 $\mathrm{mA} / \mathrm{gel}, 220 \mathrm{~V}$. Unstained protein standard (Serva) was used as a molecular weight marker.

\subsubsection{BN PAGE}

Electrophoretic separation of native membrane protein complexes was carried out using Blue-native polyacrylamide gel electrophoresis (BN PAGE) (Schägger at al., 1991). Mitochondria pellet was resuspended in detergent solubilisation buffer to a final concentration of $1 \mathrm{mg} / \mathrm{ml}$. Solubilisation was carried out for $20 \mathrm{~min}$ at $4^{\circ} \mathrm{C}$. After centrifugation at $16,000 \times \mathrm{g}$ for $10 \mathrm{~min}$ to remove the unsolubilised material, the supernatant was mixed with BN sample loading buffer. The gel system consisted of a $4 \%$ stacking gel and a $4-13 \%$ or $6-16 \%$ resolving gel. Electrophoresis was performed at $4{ }^{\circ} \mathrm{C}$. Electrophoresis conditions through the stacking gel were at $600 \mathrm{~V}, 15 \mathrm{~mA} / \mathrm{gel}$ for $1 \mathrm{~h}$, using cathode buffer with Coomassie Brilliant Blue G-250. Subsequently, the cathode buffer was exchanged to one without any dye, and the gel was run at $100 \mathrm{~V}, 15 \mathrm{~mA} / \mathrm{gel}$ for $14 \mathrm{~h}$.

\subsubsection{Coomassie staining}

Gels and PVDF membranes were stained using Coomassie staining solution for visualisation of proteins. Gels were stained for $1 \mathrm{~h}$ and membranes for 2-3 min. Following 
this, destaining solution was used until protein bands were clearly visible over the background. For staining the proteins for mass spectrometry analysis, Colloidal Coomassie staining solution was used (modified from (Neuhoff et al., 1988)). Here, the gel was first fixed in destaining solution for $2 \mathrm{~h}$ at room temperature under shaking conditions. Following this, the gel was washed 3 times for 10 min each with $\mathrm{dH}_{2} \mathrm{O}$. Staining was done using Colloidal coomassie staining solution at room temperature overnight with shaking. For destaining, $1 \%$ acetic acid was utilised.

\subsubsection{Western blotting and immunodecoration}

Western blotting (Burnette, 1981) was performed for proteins separated by SDS PAGE and BN PAGE by transferring them to polyvinylidene difluoride (PVDF) membrane using semi-dry blotting chambers (PEQLAB). Briefly, membrane with the same size as the gel was activated first using methanol. Following this, the blotting layers were assembled. This consisted of three Whatman paper soaked in buffer at the bottom, methanolactivated membrane, gel and finally three Whatman papers again. Transfer was performed at $250 \mathrm{~mA}$ for $2 \mathrm{~h}$. Following transfer, membranes were stained with Coomassie staining solution as described above. Protein standard bands were marked and the membrane was cut into parts depending on the antibodies to be used. After this, total destaining was done using methanol. The membranes were incubated in blocking solution ( $5 \%$ milk in TBST) for $1 \mathrm{~h}$. Following a brief wash with TBST, the membranes were incubated with primary antibodies at room temperature for $1 \mathrm{~h}$ or at $4^{\circ} \mathrm{C}$ overnight. Subsequently, three washing steps in TBST of 10 min each were carried out, after which the membranes were incubated in their corresponding secondary HRP-conjugated antibodies for $1 \mathrm{~h}$ at room temperature. three washing steps with TBST were repeated, following which ECL reagent (Thermo Scientific) was added and signals were detected using X-ray films.

For fractions collected after GraFix (section 2.2.4.6), dot blotting was performed. $2 \mu \mathrm{l}$ of each fraction was applied on a nitrocellulose membrane (Amersham Protran Supported $0.45 \mu \mathrm{m} \mathrm{NC)}$. After the blot was dry, blocking and subsequent antibody incubations were performed as described above.

\subsubsection{Digital autoradiography}

Samples with import of ${ }^{35} \mathrm{~S}$ labelled proteins were separated on SDS PAGE or BN PAGE. After staining and destaining with Coomassie solution, the gels were put on two Whatman papers and covered with a plastic sheet on top. Subsequently, they were dried on GD5040 Gel Dryers (Scie-Plas) for $3 \mathrm{~h}$ at $65^{\circ} \mathrm{C}$. Following this, the protein standards were 
marked with radioactive ink and covered with a transparent tape. The gels were exposed on Phosphor screens (GE Healthcare) and signals were detected using Amersham Typhoon Phosphorlmager (GE Healthcare). Quantification of signals was carried out using the ImageQuantTL software (GE Healthcare).

\subsubsection{Glycerol density gradients}

$10-30 \%$ glycerol density gradients were prepared by mixing $10 \%$ glycerol buffer and $30 \%$ glycerol buffer using pre-programmed conditions for $10-30 \%$ glycerol gradients on Gradient Master (BioComp Instruments). Gradients were cooled at $4^{\circ} \mathrm{C}$ for $2-3 \mathrm{~h}$. For GraFix (Gradient Fixation) (Kastner et al., 2008), the high density 30\% buffer contained 0.05\% glutaraldehyde. For GraDeR (Gradient Detergent Removal) (Hauer et al., 2015), the low density $10 \%$ buffer contained $0.05 \%$ GDN while the high density $30 \%$ buffer contained $0.02 \%$ GDN. After isolation, $200 \mu$ protein complexes were overlayed on the gradients, after which ultracentrifugation was carried out in Sw60Ti rotors (Beckmann Coulter) for $18 \mathrm{~h}$ at $121,262 \times \mathrm{g}$ at $4^{\circ} \mathrm{C} .0 .4 \mathrm{ml}$ fractions were collected from the top and were analysed by SDS-PAGE followed by western blotting or by dot blotting.

\subsubsection{Specialised assays}

\subsubsection{Protein import, assembly and generation of the TOM-TIM23 supercomplex}

Import of radiolabelled precursor or recombinant protein was performed (Ryan et al., 2001) as follows. Mitochondria were unfrozen on ice. A typical import reaction consisted of $50 \mu \mathrm{g}$ mitochondria suspended in import buffer (section 2.1.1.3) to have a final concentration of $1 \mu \mathrm{g} / \mu \mathrm{l}$. For import reaction longer than $15 \mathrm{~min}, \mathrm{CK}$ and $\mathrm{CP}$ were added as ATP regenerating system. In a control sample, membrane potential $(\Delta \Psi)$ was dissipated using $1 \%$ AVO mix. Import was carried out for desired time points at $25^{\circ} \mathrm{C}$ under mild shaking (450 rpm on Thermomixer $C$ ) and was terminated by addition of $1 \%$ AVO. Samples were then placed on ice. Non-imported precursors were digested by 50 $\mu \mathrm{g} / \mathrm{ml}$ proteinase $\mathrm{K}(\mathrm{PK})$ treatment for $10 \mathrm{~min}$, whenever required. PK reaction was inactivated by $2 \mathrm{mM}$ PMSF. Subsequently, mitochondria were centrifuged at $16,000 \times \mathrm{g}$ for $10 \mathrm{~min}$, followed by wash with SEM buffer. Samples were analysed by SDS PAGE or BN PAGE in combination with western blotting or autoradiography.

For radiolabelled proteins, maximum $10 \%(\mathrm{v} / \mathrm{v})$ was used for import reactions. For assembly of proteins, $12.5 \%(\mathrm{v} / \mathrm{v})$ lysate was used. For recombinant proteins, $5-100 \mu \mathrm{g}$ protein was imported for $30 \mathrm{~min}$. 
For import block assay, different amounts of recombinantly purified proteins were imported into mitochondria for $30 \mathrm{~min}$. Mitochondria were then pelleted down, washed with SEM buffer, and resuspended in fresh import buffer. This was followed by import of $6 \%{ }^{35} \mathrm{~S}$-labelled $\mathrm{F} 1 \beta$ for $15 \mathrm{~min}$. After this, mitochondria were centrifuged, washed with SEM, PK treated, loaded on SDS gels and analysed by autoradiography. After quantification of signals, data processing was carried out using DataGraph.

Generation of the TOM-TIM23 supercomplex was achieved by arresting the import of a tightly folded precursor protein (Rassow et al., 1989). b $b_{2}(167)_{\Delta}$-DHFR, SCGP, Jac1-sfGFP and Mam33-sfGFP were imported to form the supercomplex. Purified $b_{2}(167)_{\Delta}$-DHFR was provided by Dr. Christian Schulz (AG Rehling, Institute of Cellular Biochemistry, Göttingen, Germany), while the other proteins were purified as described (section 2.2.4.1). $\mathrm{b}_{2}(167)_{\Delta}$-DHFR (10 $\mu \mathrm{g} / 50 \mu \mathrm{g}$ mitochondria) was incubated with $5 \mu \mathrm{M}$ methotrexate before being imported. For the sfGFP-containing proteins, $20 \mu \mathrm{g}$ protein was added per $50 \mu \mathrm{g}$ mitochondria. Import was carried out for $30 \mathrm{~min}$ as described above.

\subsubsection{Isolation of complexes}

Two different strategies were utilised for the isolation of protein complexes: 1) Using GFP nanobody to isolate the TOM-TIM23 supercomplex generated by the import of sfGFPcontaining proteins (see section 2.2.5.1), or 2) TIM23 complex isolation using HisSUMOstar or His-Nedd8 tag on Tim23.

In the first approach, the TOM-TIM23 supercomplex was generated as described before (see section 2.2.5.1). Following import, $250 \mu \mathrm{g}$ mitochondria were centrifuged at 16,000 $\mathrm{x}$ $\mathrm{g}$ for $10 \mathrm{~min}$ at $4^{\circ} \mathrm{C}$. After washing with SEM, mitochondria were solubilised in detergent solubilisation buffer with $1 \%$ digitonin and $1 \mathrm{mM}$ PMSF (unless otherwise specified) as described previously (see section 2.2.4.3). In parallel, $25 \mu$ Strep-tactin sepharose beads (IBA) were equilibrated with $2 \times 500 \mu \mathrm{ld} \mathrm{d}_{2} \mathrm{O}$, followed by $2 \times 500 \mu \mathrm{l}$ PBS in a MobiCol. Centrifugation for these steps was carried out at $100 \times \mathrm{g}$ for $30 \mathrm{~s}$. After this, $500 \mu \mathrm{l}$ PBS containing $1 \mu \mathrm{g}$ purified GFP nanobody was incubated with the beads for $1 \mathrm{~h}$ at room temperature, on shaking. Subsequently, the beads were washed with $2 \mathrm{X}$ buffer $(40 \mathrm{mM}$ HEPES/KOH pH 7.4, $300 \mathrm{mM} \mathrm{NaCl}, 40 \%$ glycerol and $0.2 \mathrm{mM}$ EDTA) and detergent solubilisation buffer. The clarified supernatant (Total) of solubilised mitochondria was applied to the beads. Binding was done for $1 \mathrm{~h}$ at $4^{\circ} \mathrm{C}$ under shaking. Following this, the unbound sample was collected as flowthrough. The beads were washed 10x with wash buffer containing $0.3 \%$ digitonin. Elution was carried out with $7.5 \mathrm{mM}$ desthiobiotin in wash buffer (200 x g, $1 \mathrm{~min}$ ). Total, flowthrough and elution fractions were analysed by SDS 
PAGE and western blotting. Total and flowthrough were always loaded at $5 \%$ of elution unless otherwise indicated.

For the TIM23 complex isolation, His-SUMOstar-Tim23 and His-Nedd8-Tim23 yeast strains were utilised. In these strains, the endogenous Tim23 has been replaced by HisSUMOstar-Tim23 or His-Nedd8-Tim23. Mitochondria from these strains were prepared as described previously (section 2.2.2.4). $\mathrm{Ni}^{2+}$-NTA agarose beads (Macherey Nagel) were washed with $2 \times 500 \mu \mathrm{ldH} \mathrm{H}_{2} \mathrm{O}$, followed by $2 \times 500 \mu \mathrm{l} 2 \mathrm{X}$ buffer and $2 \times 500 \mu \mathrm{l}$ detergent solubilisation buffer. Solubilised mitochondria were incubated with equilibrated beads for 2-3 $\mathrm{h}$ at $4^{\circ} \mathrm{C}$ under mild shaking. Following washing in a buffer without PMSF, elution was carried out using $1 \mu \mathrm{M}$ SUMOstar or $1 \mu \mathrm{M}$ Nedd8 protease (constructs provided by Dr. Alexander Stein) in wash buffer without PMSF for $1 \mathrm{~h}$ at $4^{\circ} \mathrm{C}$. For mitochondria which were subjected to Jac1-sfGFP import, a second GFP nanobody isolation step can be added after isolation of the TIM23 complex. This two step isolation strategy leads to a specific isolation of the TOM-TIM23 supercomplex.

All isolations were scaled according to the analysis method. Samples obtained after isolation were assessed by SDS PAGE and western blotting or were applied to glycerol gradients or directly analysed by negative stain EM or cross-linking-MS.

\subsubsection{Detergent optimisation}

The TIM23 complex stability was assessed under digitonin, LMNG, GDN and SMA solubilisation. The TIM23 complex was isolated as described before (see section 2.2.5.2). Mitochondria solubilsation was carried out with detergent solubilisation buffer supplemented with $1 \%$ detergent (digitonin, LMNG or GDN) or with SMA solubilisation buffer. With detergents, solubilisation was carried out at a final concentration of $1 \mathrm{mg} / \mathrm{ml}$. With SMA, the final concentration was $8 \mathrm{mg} / \mathrm{ml}$ during solubilisation, but the sample was diluted back to $1 \mathrm{mg} / \mathrm{ml}$ before binding. The corresponding wash buffer contained $0.3 \%$ digitonin, $0.05 \%$ LMNG, $0.05 \%$ GDN or SMA wash buffer without SMA. To assess the effect of detergent exchange on stability of the complex, solubilisation was carried out with $1 \%$ digitonin. However, after the final washing step before elution, wash buffer containing either $0.05 \%$ LMNG or $0.05 \%$ GDN was applied on the beads. They were incubated for 30 min at $4^{\circ} \mathrm{C}$ under mild shaking. Elution was carried out with buffer containing $0.05 \%$ LMNG or $0.05 \%$ GDN. Samples were mixed with SDS PLD and analysed by SDS PAGE and western blotting. 


\subsubsection{Negative staining}

For visualisation of the isolated TOM-TIM23 supercomplex by electron microscopy (EM), complexes were prepared by negative staining in collaboration with Dr. Niels Fischer (Department of Structural Dynamics, Max Planck Institute for Biophysical Chemistry, Göttingen, Germany) (Stark and Chari, 2016). Samples were applied onto EM grids in two different ways: i) $\sim 5 \mu \mathrm{l}$ drops of complex solution were directly applied to glow-discharged EM grids covered with a continuous carbon film, or ii) $\sim 30 \mu$ complex solution were applied into the hole of a Teflon block, carbon film was floated onto the solution and incubated thereon for defined time-intervals. After sample application, EM grids were blotted with pre-wetted Kim-Wipes paper, excess detergent was diluted by incubating grids three times on $\sim 20 \mu$ l buffer drops, each time followed by blotting with the pre-wetted Kim-Wipes paper. Finally, samples were stained by floating grids onto $2 \%(\mathrm{w} / \mathrm{v})$ solution of uranyl formate for 30 seconds, and excess stain was blotted using the pre-wetted KimWipes paper. EM images were recorded on a $4 \mathrm{kx} 4 \mathrm{k}$ CCD Camera (2x binning, TVIPS $\mathrm{GmbH}$, Gauting, Germany) in a Philips CM200 FEG (Thermo Fisher Company, Eindhoven, The Netherlands), at nominal magnifications of $88 \mathrm{k}$ and $115 \mathrm{k}$, corresponding to pixel sizes of about $1.85 \AA / p x$ and $2.5 \AA / p x$, respectively.

\subsubsection{Cross-linking analysis}

The two step isolated TOM-TIM23 supercomplex was subjected to cross-linking with 500 $\mu \mathrm{M}$ of DSS, DSG, BS2G and BS3 for $30 \mathrm{~min}$ on ice. Same amount of supercomplex without any treatment of cross-linker was also kept on ice. Subsequently, the cross-linking reaction was quenched by $250 \mu \mathrm{M}$ glycine $\mathrm{pH} 8.0$ for $15 \mathrm{~min}$ on ice. SDS PLD was added and the samples were analysed by SDS-PAGE and western blotting. For cross-linking followed by mass spectrometry, the TIM23 complex was isolated after import of buffer or Jac1-sfGFP. The isolated complex was treated with $1 \mathrm{mM}$ DSS for $3 \mathrm{~h}$ on ice, followed by quenching of the reaction with $250 \mu \mathrm{M}$ glycine $\mathrm{pH} 8.0$ for $30 \mathrm{~min}$. Proteins were precipitated overnight at $-20^{\circ} \mathrm{C}$ using $80 \%$ acetone. The following day, samples were centrifuged. Mass spectrometry analysis was carried out by Andreas Linden (Research Group Bioanalytical Mass Spectrometry, Max Planck Institute for Biophysical Chemistry, Göttingen, Germany). Analysis of cross-links obtained was carried out using XVis (Grimm et al., 2015) and PyMol (The PyMOL Molecular Graphics System, Version 2.0 Schrödinger, LLC). 


\subsubsection{Quantitative proteomics of mitochondria}

WT and tim22-14 cells were cultured in $5 \mathrm{ml}$ YPD media at $24^{\circ} \mathrm{C}$. Subsequent cultures were done in $50 \mathrm{ml}, 300 \mathrm{ml}$ and $2 \mathrm{~L}$ YPG. After growing the $2 \mathrm{~L}$ culture at $24^{\circ} \mathrm{C}$ till $\mathrm{OD}_{600}$ of $1-1.5$, the culture was split into $4 \times 500 \mathrm{ml}$ and incubated at $37^{\circ} \mathrm{C}$ for $0 \mathrm{~h}, 15 \mathrm{~h}, 25 \mathrm{~h}$ and $40 \mathrm{~h}$. Crude mitochondria were prepared as mentioned above (section 2.2.2.4). $10 \mu \mathrm{g}$ of mitochondria from all conditions were reduced, alkylated and trypsin digested by Dr. Ida Suppanz (Biochemistry and Functional Proteomics, University of Freiburg, Freiburg, Germany). Mass spectrometry based proteomic analysis was also carried out by her. Briefly, the peptides were labelled with light, medium or heavy dimethyl. WT peptides from all time points received light labelling, tim22-14 peptides at $0 \mathrm{~h}$ time point received medium and tim22-14 peptides at time points 15,25 and $40 \mathrm{~h}$ of heat shock received heavy labelling. Each sample contained WT (light) and tim22-14 (heavy) peptides for a specific heat shock time point along with the tim22-14 $0 \mathrm{~h}$ (medium) peptides. Heavy/light ratio was calculated to identify down-regulated proteins in heat- shocked mitochondria. 


\section{Results}

\subsection{Analysis of a mitochondrial translocation intermediate: the TOM-TIM23 supercomplex}

\subsubsection{Design and comparison of new supercomplex forming proteins}

Mitochondrial preproteins form transient translocation contact sites during their import, spanning both the TOM and TIM23 complexes. The formation of a TOM-TIM23 supercomplex has been demonstrated before (Koll et al., 1992) (Dekker et al., 1997) (Chacinska et al., 2003). This was accomplished with the help of model protein $b_{2}(167)_{\Delta^{-}}$ DHFR, which consists of the N-terminal part of cytochrome $b_{2}(1-167$, with deletion of 19 amino acid in the sorting signal between residues 47-65) fused to dihydrofolate reductase (DHFR). DHFR folds stably in the presence of methotrexate (MTX), preventing complete entry of the protein into mitochondria. However, the inability of $b_{2}(167)_{\Delta}-$ DHFR to be purified to a high concentration leads to it not being utilised to its full potential of $100 \%$ saturation of the translocases for supercomplex formation. A new set of fusion proteins were designed to test if the efficiency of supercomplex formation and isolation can be improved. The three main components that such a fusion protein should have are: (i) $A$ presequence which gets recognised by mitochondrial translocase receptors, (ii) a spacer domain which spans both the mitochondrial membranes and (iii) a blocking moiety which arrests the protein at the translocation contact site, leading to formation of the TOM-TIM23 supercomplex (Figure 8). Three new proteins were designed, all containing superfolder GFP (sfGFP) as the blocking moiety at the C-terminus (Pédelacq et al., 2006). Since the diameter of GFP (2.4 nm) (Hink et al., 2000) is slightly bigger than that reported for the TOM complex pore (1.1 nm short vs $3.2 \mathrm{~nm}$ long (Bausewein et al., 2017) or $2.2 \mathrm{~nm}$ estimated average (Hill et al., 1998)), it would get arrested on the cytoplasmic face of the TOM complex. The three proteins were: (i) Supercomplex generating protein (SCGP), containing the presequence of Atp2 and a spacer region from membrane scaffold protein 1E3D1 (ii) Jac1-sfGFP, which had the presequence and spacer from the mitochondrial 
matrix protein Jac1, and (iii) Mam33-sfGFP, containing the presequence and spacer from the mitochondrial matrix protein Mam33 (Figure 8).

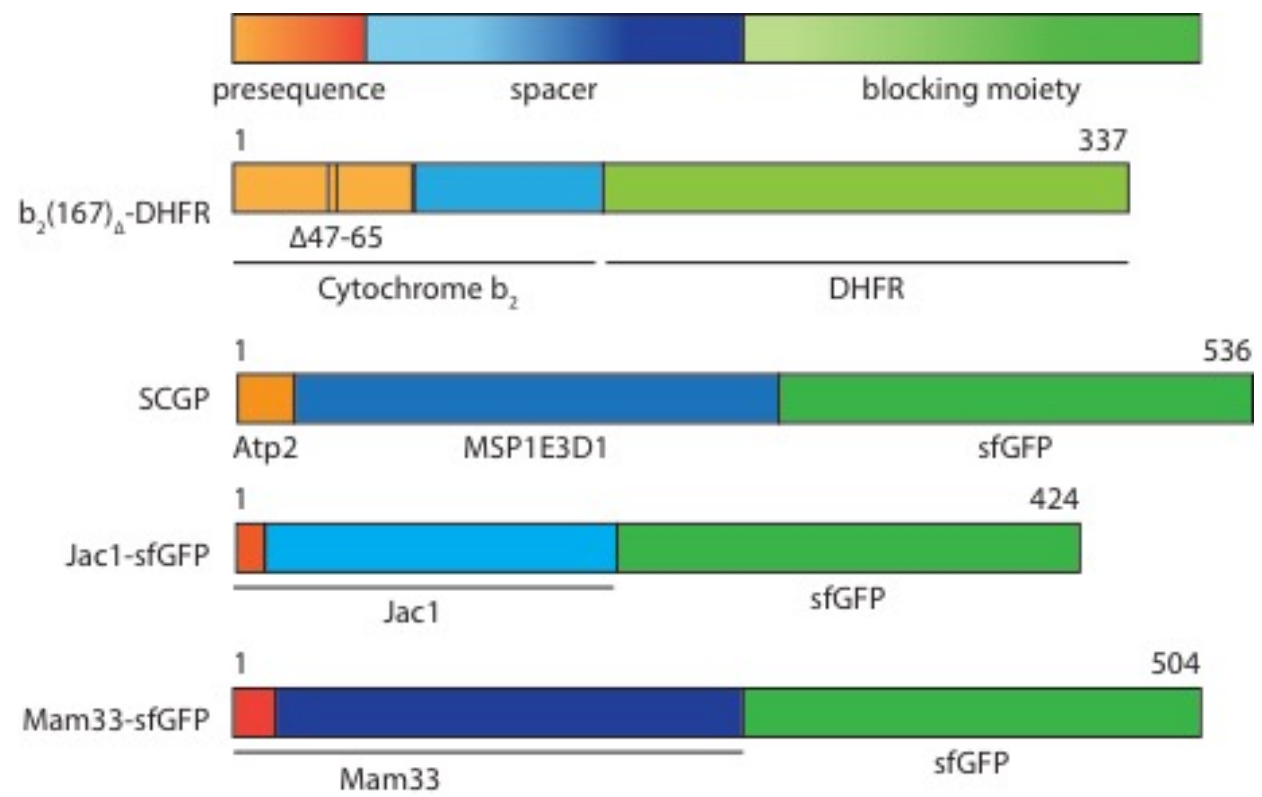

Figure 8 Design of supercomplex forming proteins - The supercomplex forming proteins are based on the ability of the protein to span both the inner and the outer mitochondrial membrane while being tightly folded at the $\mathrm{C}$-terminus. This protein should have a $\mathrm{N}$-terminal presequence, a spacer region to span both the membranes and a blocking moiety to prevent complete translocation of the protein into mitochondria. Proteins used in this study are:- fusion protein $b_{2}(167)_{\Delta}$ - DHFR: presequence and spacer - cytochrome $b_{2}$ with 19 amino acid sorting signal deletion $(\Delta)$, blocking moiety: mouse DHFR; SCGP (SuperComplex Generating Protein): presequence - Atp2, spacer - membrane scaffold protein 1E3D1, blocking moiety - sfGFP; Jac1sfGFP: presequence and spacer - Jac1, blocking moiety - superfolder GFP (sfGFP); Mam33sfGFP: presequence and spacer - Mam33, blocking moiety - superfolder GFP (sfGFP). Red: presequence; blue: spacer; green: blocking moiety.

\subsubsection{1 in vivo expression of protein shows localisation to mitochondria}

Newly constructed fusion proteins have to be import competent to form the supercomplex. To that end, the fluorescence property of sfGFP was utilised to track the localisation of these proteins in S. cerevisiae. Plasmids encoding SCGP or Jac1-sfGFP under galactose promoter were transformed into BY4741 cells. Expression was induced with galactose. Whole cell lysate of SCGP transformed cells showed increasing levels of protein (Figure 9A, lanes 4,6,8 and 10) with increasing induction time. The corresponding control Mic10 levels remained the same. The expression and localisation of SCGP and Jac1-sfGFP upon galactose induction at different time points was also observed by fluorescence microscopy. Both proteins showed localisation to mitochondria in the FITC channel for GFP, and co-localised with the membrane potential dependent MitoTracker dye (TRITC channel), as can be seen in the Merge column (Figure 9B). 
A

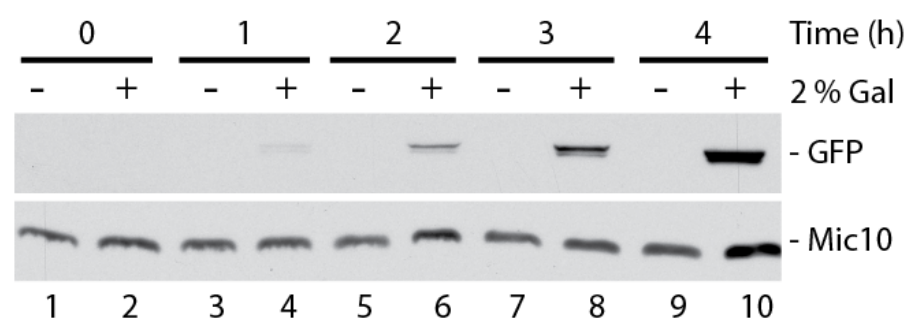

B

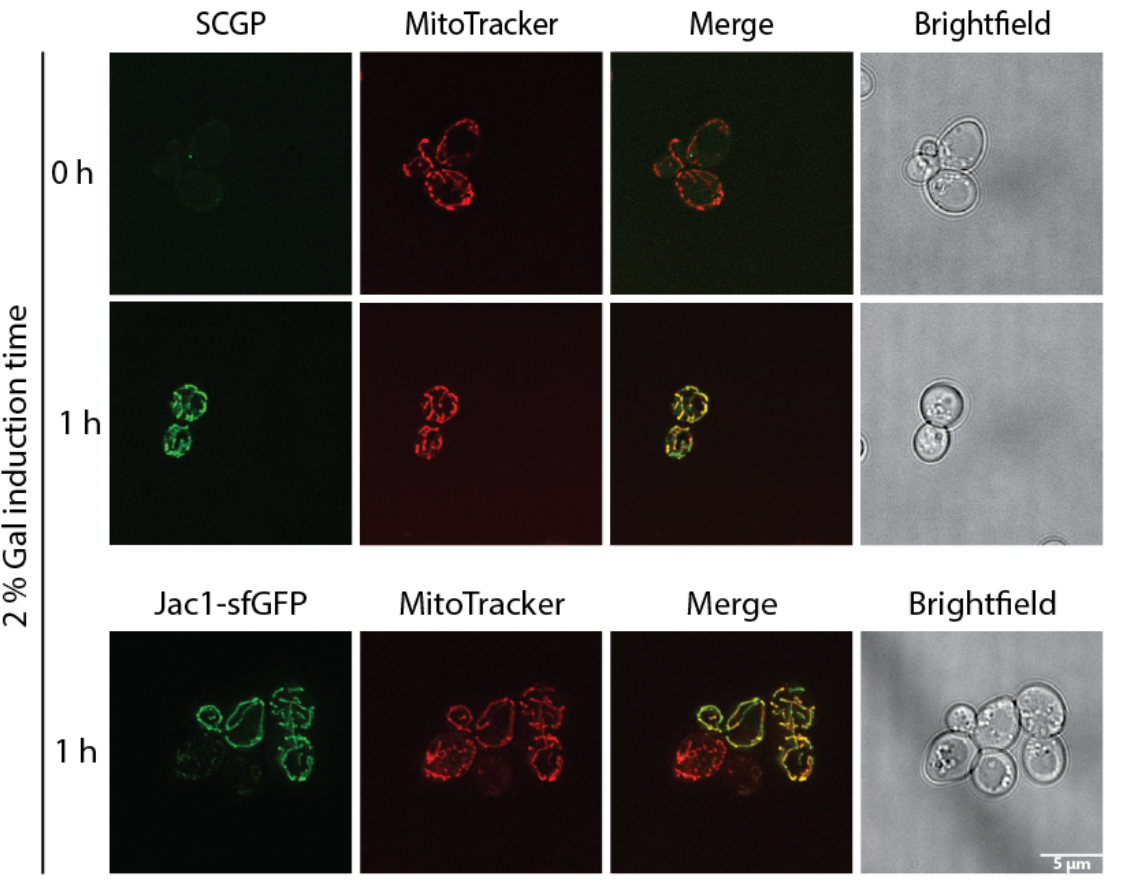

Figure 9 Supercomplex forming proteins localise to mitochondria - A. Whole cell lysate of yeast cells transformed with plasmids encoding SCGP under galactose promoter. Cells were grown in selective sucrose media followed by induction with $2 \%$ galactose. Samples were taken 0 , $1,2,3$ and $4 \mathrm{~h}$ after induction. Cell lysates were analysed by SDS-PAGE and western blotting. $\mathbf{B}$. Yeast cells were transformed with SCGP (upper panel) and Jac1-sfGFP (lower panel) encoding plasmids as in A. After induction of expression, cells were co-stained with MitoTracker Red, a fluorescent dye. Cells were viewed with a fluorescent microscope using FITC and TRITC channels. Merged green and red fluorescence images are shown (yellow/orange). (Scale bar: $5 \mu \mathrm{m}$ )

To obtain more insight into the localisation of Jac1-sfGFP, super-resolution STED microscopy was carried out for yeast cells transformed with Jac1-sfGFP plasmid. Antibody against Tom40, a mitochondrial outer membrane protein, was used to stain for mitochondria. Two-colour STED imaging showed Jac1-sfGFP co-localising with clusters formed by Tom40 (Figure 10). From these images, it can be concluded that the new fusion proteins SCGP and Jac1-sfGFP localise to mitochondria upon expression in yeast cells. However, because of toxic effects stemming from expressing the proteins for too long in the cells, which occur due to the block of mitochondrial protein translocases, and therefore import, this approach was not used for purification of the supercomplex. Instead, an in organello approach was used. 


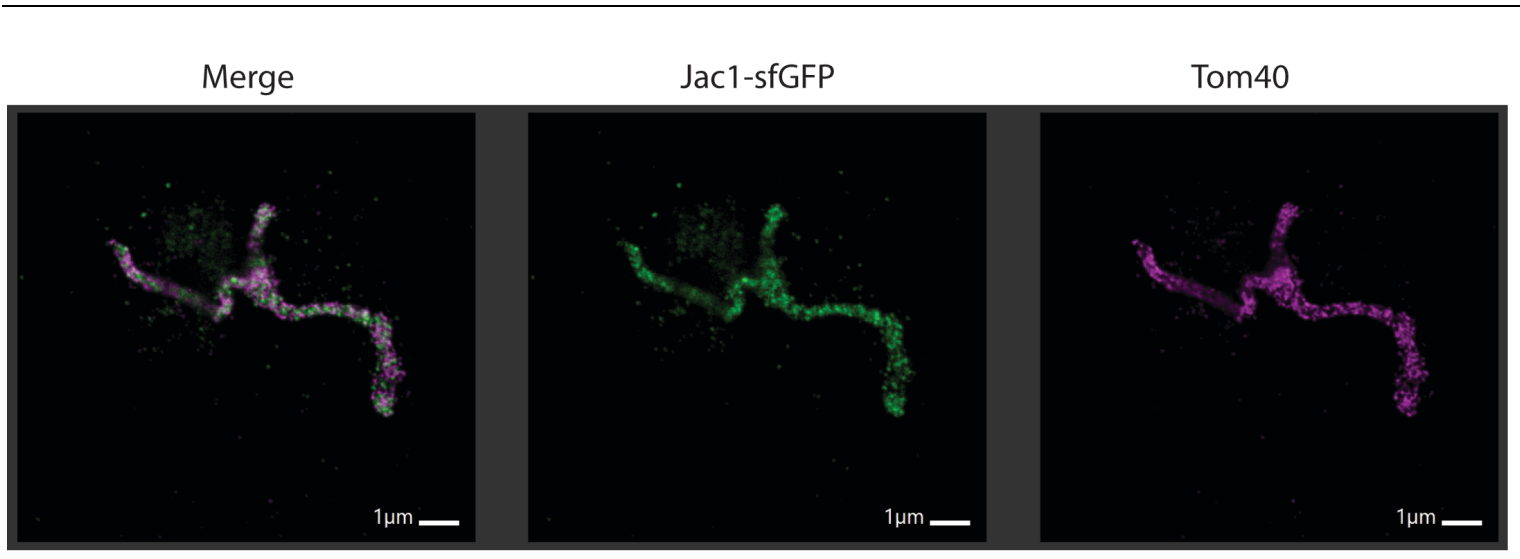

Figure 10 Super-resolution microscopy of Jac1-sfGFP - Two colour STED microscopy of yeast cells transformed with Jac1-sfGFP (green). Cells were labelled with antibody against outer membrane protein Tom40 (magenta). Merged images are shown on the left. (Scale bar: $1 \mu \mathrm{m}$ ). Images acquired by Dr. Stefan Stoldt, Research Group Mitochondrial Structure and Dynamics, Göttingen, Germany.

\subsubsection{New fusion proteins form the supercomplex to varying levels}

The TOM-TIM23 supercomplex formation has previously been reported with $b_{2}(167)_{\Delta^{-}}$ DHFR. To test the efficacy of the new proteins, they were expressed in E. coli under IPTG induction. Purification was accomplished using affinity and size exclusion chromatography. The His-SUMO tag at the $\mathrm{N}$-terminus was cleaved off using SUMO protease (Figure 11A). All three proteins showed a different running profile in size exclusion chromatography. Mam33-sfGFP showed a strong tendency to oligomerise, with almost $100 \%$ of the protein running as an oligomer. SCGP was running as a mixture of monomer and dimer. This could be explained by the presence of membrane scaffold protein (MSP) 1E3D1 in the protein. MSPs are amphipathic proteins which are used in the formation of nanodisc, and have a tendency to form discoidal belts.
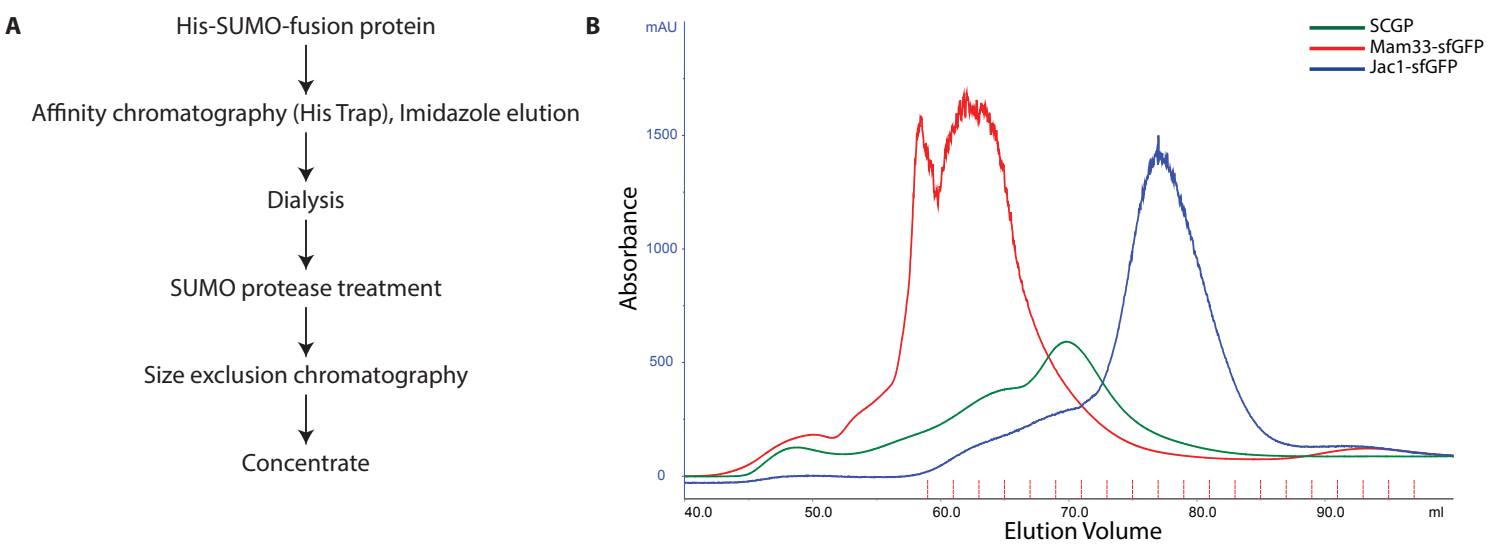

Figure 11 Purification of supercomplex forming proteins shows tendency to oligomerise - A. Scheme of the protein purification setup. N-terminally His-SUMO tagged proteins were expressed in E. coli and purified using NiNTA beads and SUMO protease treatment, followed by size exclusion chromatography. B. Chromatogram of SCGP (green), Mam33-sfGFP (red) and Jac1-sfGFP (blue) after size exclusion chromatography. Absorbance scaled for representative purpose. 
Jac1-sfGFP ran mostly as a monomer. Elution profile of a standard protein mixture applied on the column was used as a reference for size of the protein and its running behaviour (Figure 11B).

A
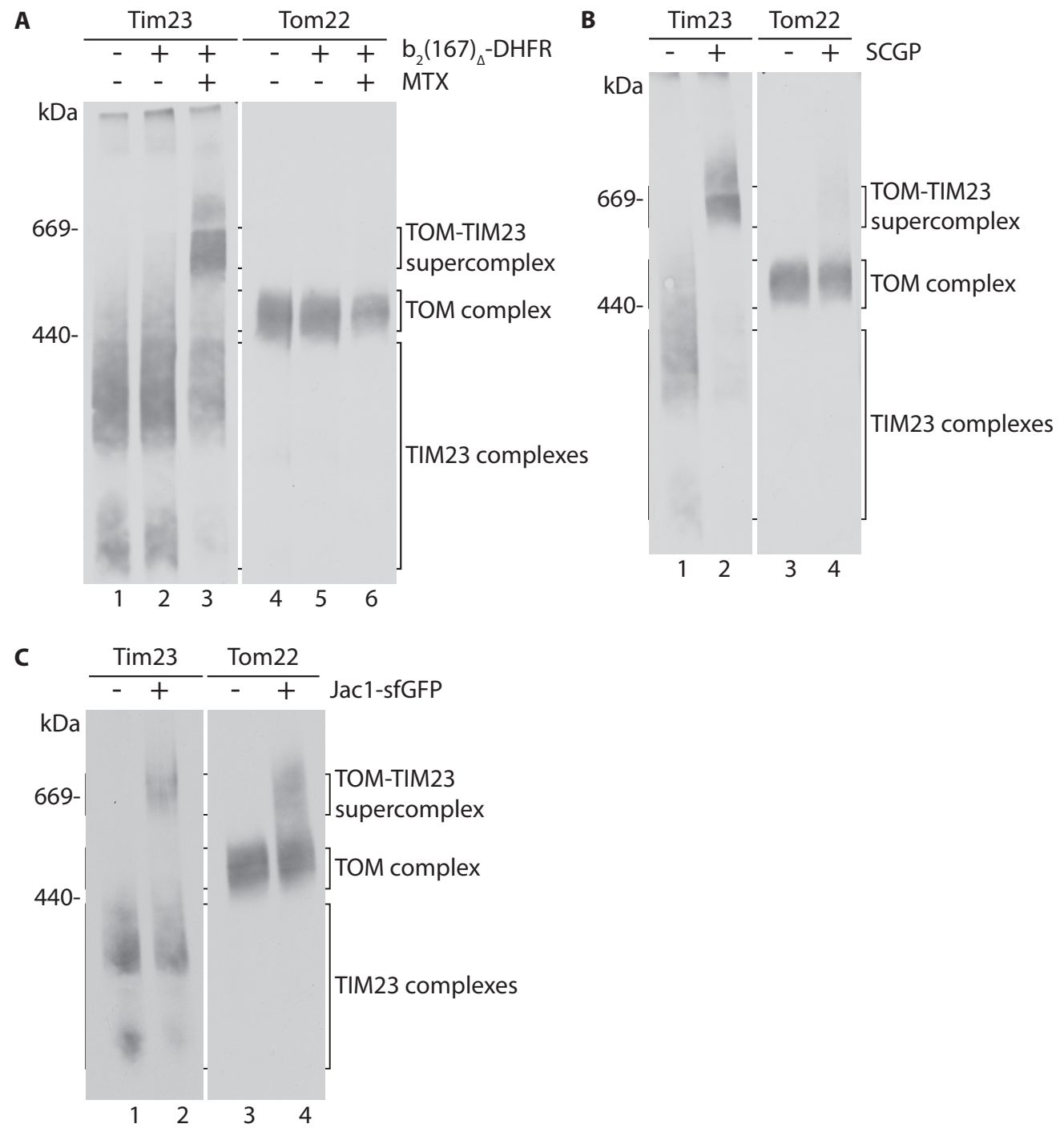

Figure 12 Chemical amounts of SCGP and Jac1-sfGFP form supercomplex - A. Purified $\mathrm{b}_{2}(167)_{\Delta}$-DHFR was imported into isolated WT mitochondria in the absence or presence of methotrexate (MTX) for $30 \mathrm{~min}$ at $25^{\circ} \mathrm{C}$. Mitochondria were then solubilised using digitonin and samples were analysed by BN-PAGE followed by western blotting using $\alpha$ Tom22 and $\alpha \operatorname{Tim} 23$ serum. B. Import of SCGP, as described in A. C. Import of Jac1-sfGFP, as described in A.

The supercomplex forming efficiency of these purified proteins was visualised on $\mathrm{BN}$ PAGE. $b_{2}(167)_{\Delta}$-DHFR was imported in the presence or absence of methotrexate. SCGP and Jac1-sfGFP were imported directly. Samples were solubilised using digitonin, and applied on BN-PAGE. In the absence of the precursor, the TIM23 complex was detected as two separate complexes with Tim23 antibody - a higher molecular weight sorting form 
and a lower molecular weight core form (Figure 12A, lane 1 and 2). Addition of the precursor in the presence of MTX led to a shift to the higher molecular weight supercomplex form (Figure 12A, lane 3) around $600 \mathrm{kDa}$. Similar upshifted complexes were detected in the presence of SCGP (Figure 12B, lane 2) and Jac1-sfGFP (Figure 12C, lane 2). The TOM complex also showed a corresponding co-migrating complex at $600 \mathrm{kDa}$ (Figure 12B and C, lane 4). However, only a fraction of the TOM complex was shifted into the higher supercomplex form, which can be expected since it has already been demonstrated that there are $4 \mathrm{X}$ more copies of the TOM complex than the TIM23 complex (Dekker et al., 1997) (Morgenstern et al., 2017). Therefore, both SCGP and Jac1-sfGFP can successfully generate the supercomplex.

\subsubsection{Accumulation of supercomplex forming proteins at the translocase inhibits import of mitochondrial proteins}

In order to compare the degree of translocase arrested and amounts of protein required to saturate the TIM23 complex import sites, increasing amount of the purified proteins were arrested for $30 \mathrm{~min}$. Following reisolation and resuspension of mitochondria in import buffer, radiolabelled mitochondrial matrix protein F1 $\beta$ was imported for 15 min. Decrease in F1 $\beta$ import could be observed with increasing levels of proteins accumulated at the translocase (Figure 13A,B,C,D - mature 'm' form). As expected, absence of $\Delta \Psi$ abolished import of the protein (Figure 13A,B, lane 9, C,D lane 10). All proteins also behaved differently in their ability to saturate the import sites. $b_{2}(167)_{\Delta}$-DHFR reached $50 \%$ saturation at about $8 \mu \mathrm{g}$ protein/ $50 \mu \mathrm{g}$ mitochondria. SCGP looked the most promising, with $4 \mu \mathrm{g}$ protein required per $50 \mu \mathrm{g}$ mitochondria to reach $50 \%$ saturation. Jac1-sfGFP required around $11 \mu \mathrm{g}$ and Mam33-sfGFP $50 \mu \mathrm{g}$ per $50 \mu \mathrm{g}$ mitochondria (Figure 13E). Mam33-sfGFP therefore does not form supercomplex as efficiently as the other proteins. Since SCGP, Jac1-sfGFP and Mam33-sfGFP were purified in the same conditions, the difference in import could be due to the presequence and spacer regions of these proteins. Atp2 of SCGP and Jac1 presequence could have a stronger affinity for the translocase receptors than Mam33, leading to a faster import. The inability of $b_{2}(167)_{\Delta^{-}}$ DHFR to be purified to a higher concentration leads to its not being utilised to its full potential of $100 \%$ saturation. Therefore, further experiments were carried out with SCGP, Jac1-sfGFP and Mam33-sfGFP. 
A

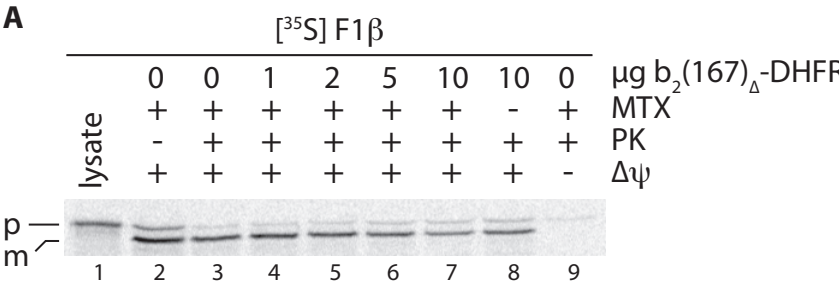

B

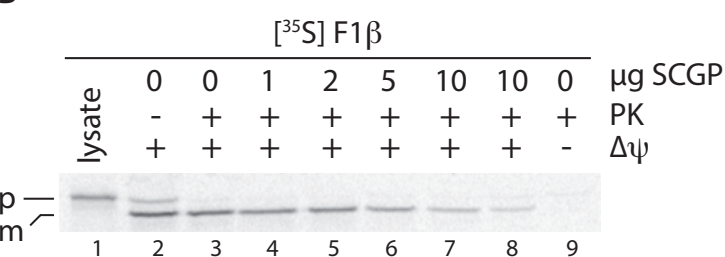

D

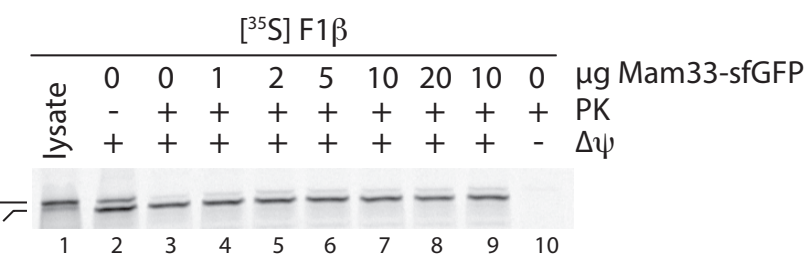

\section{E}

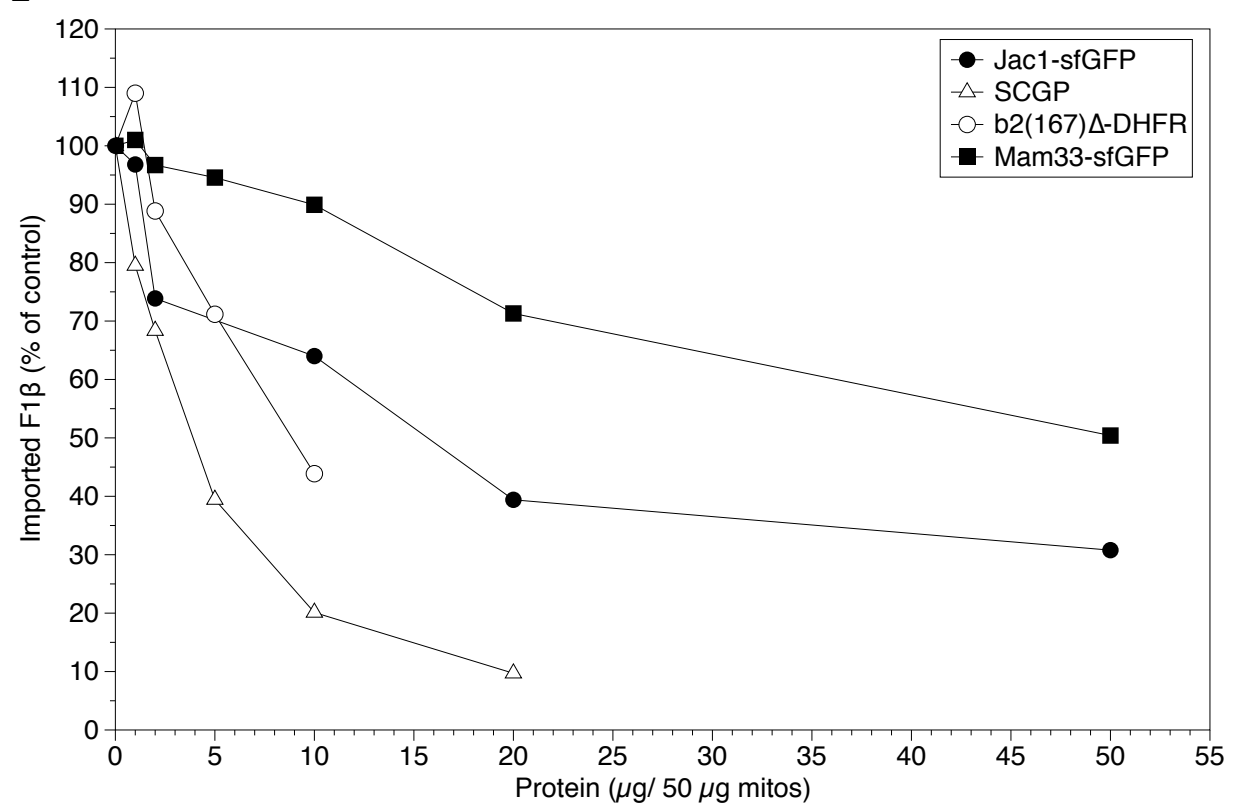

Figure 13 Supercomplex forming proteins block presequence import - Recombinantly purified proteins A. $b_{2}(167) \Delta-D H F R$, B. SCGP, C. Jac1-sfGFP and D. Mam33-sfGFP block the mitochondrial translocase upon import, preventing the import of presequence proteins. Briefly, indicated amount of fusion protein was imported per $50 \mu \mathrm{g}$ isolated WT mitochondria for $30 \mathrm{~min}$ at $25^{\circ} \mathrm{C}$. Mitochondria were briefly washed and $\left[{ }^{35} \mathrm{~S}\right]$ labelled $\mathrm{F} 1 \beta$ was imported for $30 \mathrm{~min}$. The reaction was stopped by addition of AVO followed by PK treatment. Samples were analysed by SDS-PAGE and autoradiography. E. Quantification of the autoradiogram. Imported precursor was quantified as percent of the $0 \mu \mathrm{g}$ protein sample with PK treatment $(100 \%$ control) (lane 3$)$. p precursor, $\mathrm{m}$ - mature (processed) form, AVO - mixture of $10 \mathrm{mM}$ Antimycin A, $1 \mathrm{mM}$ Valinomycin and $10 \mathrm{mM}$ Oligomycin.

\subsubsection{The TOM-TIM23 supercomplex isolation with GFP Nanobody (Nb)}

The import and subsequent supercomplex formation by SCGP and Jac1-sfGFP could be visualised by BN-PAGE analysis. These proteins are tightly held at the C-terminus outside the TOM complex by their blocking moiety and at the $\mathrm{N}$-terminus in the matrix by motor proteins. To analyse stability of the supercomplex and its subunits, isolation assays were performed. SCGP, Jac1-sfGFP and Mam33-sfGFP were imported into mitochondria. They 
were subsequently lysed, and the supercomplex was isolated with the help of Strep tagGFP Nanobody (GFP Nb) by affinity chromatography (Figure 14A). For both SCGP and Jac1-sfGFP import, similar levels of TOM (Tom40, Tom22), TIM23 (Tim23, Tim50, Tim17, Tim21) and PAM (Tim44, Hsp70) complex components could be isolated (Figure 14B, lane 6 and 7). Control proteins such as Pam17 and Aco1 were not co-isolated with the supercomplex. Mam33-sfGFP did not import efficiently, therefore the translocase proteins could only be faintly detected (Figure 14B, lane 8). As expected, the control column with no protein imported did not co-isolate any proteins (Figure 14B, lane 5).

A

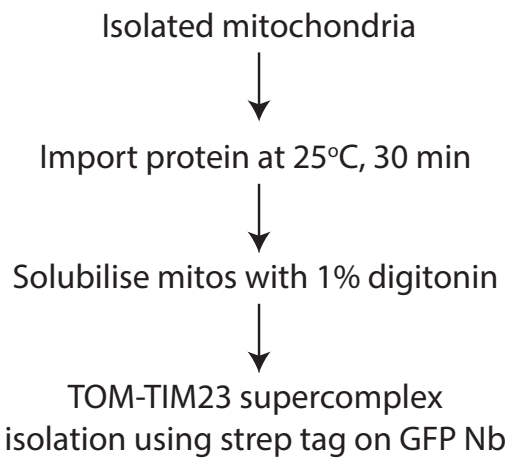

B
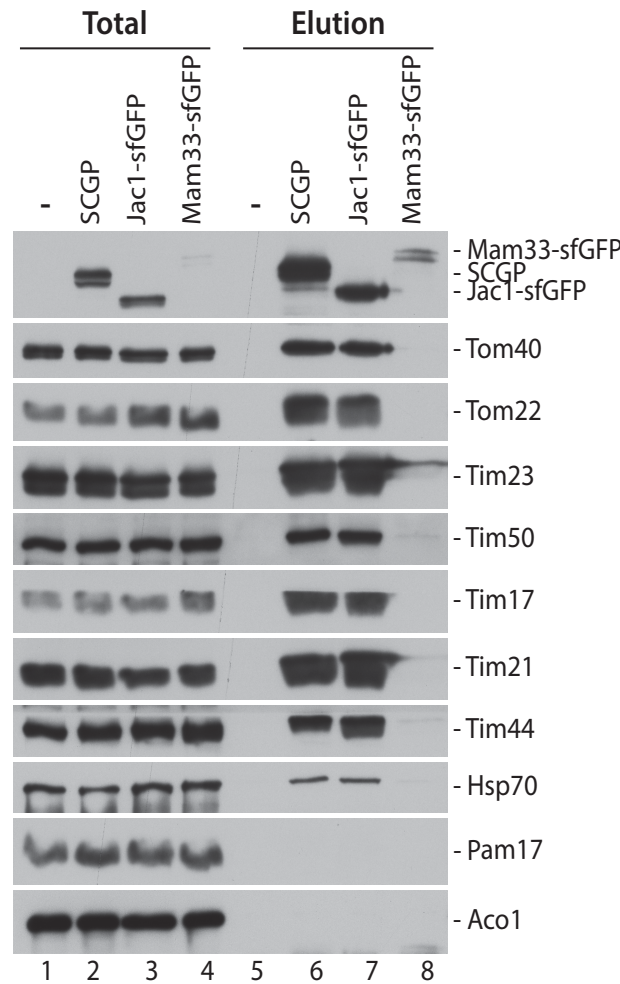

Figure 14 The TOM-TIM23 supercomplex can be isolated using GFP Nb - A. Scheme showing strategy for the TOM-TIM23 supercomplex isolation. Recombinantly purified protein was imported into isolated mitochondria for $30 \mathrm{~min}$ at $25^{\circ} \mathrm{C}$. Mitochondria were then solubilised with digitonin. Purified GFP Nb with strep tag was incubated with strep-tactin sepharose beads at RT for $1 \mathrm{~h}$. Solubilised mitos were applied to these beads, followed by elution of the complex with D-biotin. B. Import of buffer (-), SCGP, Jac1-sfGFP and Mam33-sfGFP followed by isolation as described in A. Samples were analysed by SDS-PAGE and western blotting. Total $-5 \%$, Elution $-100 \%$.

To summarise, out of the three new fusion proteins, Mam33-sfGFP did not purify as a monomer and therefore could not be used for supercomplex formation. SCGP and Jac1sfGFP seemed to work on all counts. The higher arrest of the translocase import by SCGP could be visualised by both BN-PAGE and subsequent import of radiolabelled matrix protein (Figure 12B and Figure 13B). So, it is likely that it engages more TIM23 complex with the TOM complex to form the supercomplex. However, due to its tendency to 
dimerise and oligomerise during purification (Figure 11B, green), as well as the tendency to degrade upon freeze storage, it was no longer a viable option for further experiments. Jac1-sfGFP was used in the ensuing experiments (Table 13).

Table 13 Summary of different supercomplex-forming proteins tested. 4 proteins were tested for their ability to be purified from E. coli and form the TOM-TIM23 supercomplex in isolated mitochondria.

\begin{tabular}{|c|c|c|c|c|c|}
\hline Protein & $\begin{array}{l}\text { Mol. Wt. } \\
\text { (kDa) }\end{array}$ & Expression & Purification & Import & $\begin{array}{l}\text { Supercomplex } \\
\text { isolation }\end{array}$ \\
\hline $\begin{array}{l}\mathrm{b}_{2}(167)_{\Delta^{-}} \\
\text {DHFR }\end{array}$ & 37.07 & & $\mathcal{I}$ & & \\
\hline SCGP & 58.9 & & $\sqrt{ }$ & $\sqrt{ }$ & $\sqrt{ }$ \\
\hline Jac1-sfGFP & 46.6 & $\sqrt{ }$ & $\sqrt{ }$ & $\sqrt{ }$ & $\sqrt{ }$ \\
\hline $\begin{array}{l}\text { Mam33- } \\
\text { sfGFP }\end{array}$ & 55.4 & & $X$ & $X$ & $X$ \\
\hline
\end{tabular}

\subsubsection{Optimisation of supercomplex isolation conditions}

\subsubsection{SUMOstar and Nedd8 tags on Tim23 are suitable for native isolation of the TIM23 complex}

The use of Tim23 with Protein A tag at the N-terminus (Tim23PA) for the TIM23 complex and supercomplex isolation has already been established (Geissler et al., 2002) (Chacinska et al., 2003). In order to define new conditions for more efficient isolation of the complex under native condition, a plasmid with His-SUMOstar or His-Nedd8 tag at the $\mathrm{N}$-terminus of Tim23 was introduced in S. cerevisiae after shuffling out the endogenous TIM23. SUMOstar is a variant of the SUMO protein which is not cleaved by its endogenous protease Ulp1 (Peroutka et al., 2008) and can be used for on- or postcolumn protein purification using its corresponding protease SUMOstar (Ulp1*) (Frey and Görlich, 2015). The resulting yeast cells did not show any growth defect. Mitochondria were isolated from these cells and solubilised with digitonin. The TIM23 complex was purified using Ni-NTA sepharose and SUMOstar or Nedd8 protease. Western blotting was carried out to identify co-purifying proteins. With both proteases, the amount of TIM23 complex isolated was similar (Figure 15A, lane 6 and 8). Known TIM23 complex subunits such as Tim50 and Tim21 could be co-isolated, while the corresponding control protein Aco1 was not detected. Not surprisingly, no TOM complex protein was detected in the absence of fusion protein. Upon import and arrest of SCGP before solubilisation, subunits of the TOM complex such as Tom40 and Tom22 could also be co-isolated with the TIM23 complex (Figure 15A, lane 5 and 7). GFP antibody showed the co-purified SCGP. 
Therefore, both tags displayed similar properties in terms of cell growth, mitochondrial function and complex isolation. SUMOstar tag and protease was chosen as the option to go forward with, because of better purification of SUMOstar protease compared to Nedd8 protease. An arrested preprotein could lead to specific isolation of the TOM-TIM23 supercomplex in a stable manner. This single step purification can be combined with GFP $\mathrm{Nb}$ based purification, to specifically isolate the supercomplex from mitochondria.

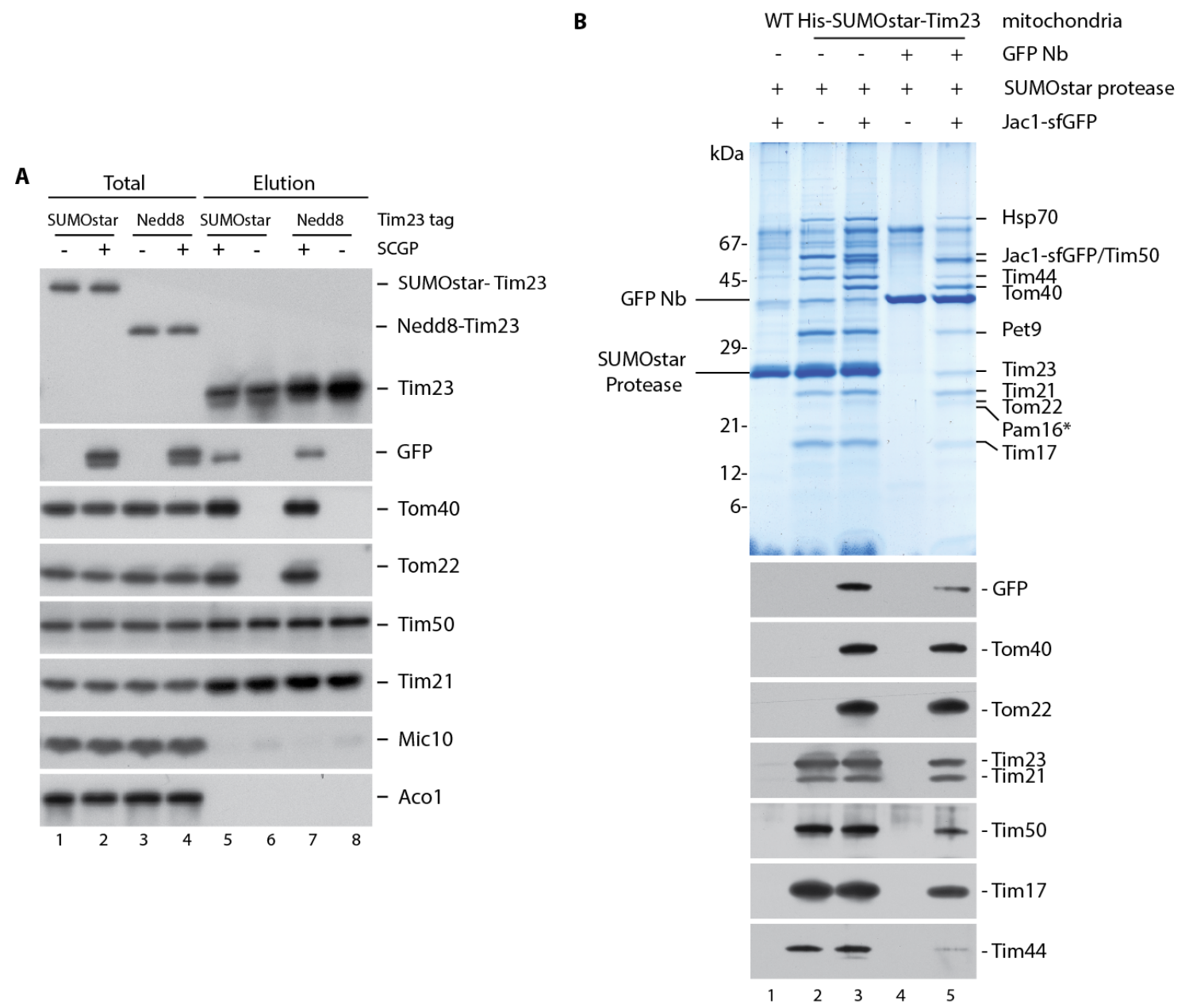

Figure 15 His-SUMOstar and His-Nedd8 tag on Tim23 helps in specific isolation of the TIM23 complex - A. Yeast strains expressing N-terminal His-SUMOstar or His-Nedd8 tagged Tim23 were generated. Mitochondria prepared from them were used for the TIM23 complex isolation. Briefly, mitochondria were subjected to import of buffer (-) or SCGP (+) as described previously. The TIM23 complex was purified by Ni-NTA affinity chromatography using SUMOstar or Nedd8 protease. Samples were analysed using SDS-PAGE and western blotting. Total - $5 \%$, Elution - $100 \%$. B. Large scale isolation of the TIM23 complex and the TOM-TIM23 supercomplex. Jac1-sfGFP was used for forming the supercomplex. The TIM23 complex was isolated as in A. Control purification from corresponding wild type mitochondria was carried out according to the same procedure. The TOM-TIM23 supercomplex was specifically isolated by following the first step of purification with isolation using GFP Nb. Samples were analysed using gradient gels. Colloidal Coomassie staining of the purified complexes and the corresponding results of mass-spectrometric analysis and western blotting analysis of the eluted proteins are shown. * Protein identified only by western blotting. 
To that end, Jac1-sfGFP was imported into WT-Tim23 and His-SUMOstar-Tim23 mitochondria, which were subsequently lysed. A first step of purification of the TIM23 complex was performed with SUMOstar protease. In the WT-Tim23 mitochondria control, only the non-specifically associated proteins could be seen on the Coomassie stained gel, while the corresponding western blot control was clean (Figure 15B, lane 1). The TIM23 complex and associated proteins could be purified from His-SUMOstar-Tim23 mitochondria (Figure 15B, lane 2 and 3). In the presence of Jac1-sfGFP (lane 3), specific bands of Tom40 and Jac1-sfGFP could be identified on the Coomassie stained gel as well as in the western blot analysis. To further enrich the supercomplex, a second step with GFP Nb was added. In the absence of Jac1-sfGFP, only the GFP Nb and non-specific bands were observed (Figure 15B, lane4). In the presence of Jac1-sfGFP, only proteins specifically present in the supercomplex could be isolated (Figure 15B, lane 5). These were confirmed by western blotting and mass spectrometry analysis. Therefore, the supercomplex can be purified with only GFP $\mathrm{Nb}$ or in combination with His-SUMOstarTim23 mitochondria.

\subsubsection{Optimisation of solubilisation conditions for the TIM23 complex purification}

To date, the TIM23 complex is only known to be stable in mild detergent digitonin. However, previous EM analysis of the complex showed a strong detergent background \{unpublished data, (Lytovchenko $O, 2012$ )\}, even when using re-crystallised digitonin (Herrmann et al., 2001). This led to diminished signal to noise ratio, affecting analysis of the particles obtained by negative staining EM. Exchanging or replacing digitonin during the supercomplex purification might be a viable option.

To that end, two new detergents were tested. One was an amphiphile called LMNG (Lauryl Maltose Neopentyl Glycol) (Chae et al., 2010) and other was a synthetic form of digitonin, known as GDN (Glyco-Diosgenin) (Chae et al., 2012). Both have been reported to solubilise and stabilise various integral membrane proteins (IMP's). Also, compared to digitonin ( $<0.5 \mathrm{mM}$ or $0.0625 \mathrm{wt} \%)$, both LMNG $(10 \mu \mathrm{M}$ or $0.001 \mathrm{wt} \%)$ and GDN (18 $\mu \mathrm{M}$ or $0.002 \mathrm{wt} \%$ ) have a lower Critical Micellar Concentration (CMC). Jac1-sfGFP was imported into mitochondria. After this, solubilisation was carried out in $1 \%$ digitonin, LMNG or GDN. Two of the digitonin solubilised samples were also subjected to detergent exchange (to $0.05 \%$ ) during the washing step of isolation. Elution was carried out using GFP $\mathrm{Nb}$. In mitochondria extracted and washed with digitonin, most of the TOM (Tom40, Tom22) and TIM23 (Tim23, Tim50, Tim17, Tim21) complex proteins could be obtained, along with motor protein Tim44 (Figure 16A, lane 6). Control proteins Aco1, Atp20 and 
Mic10 were not co-isolated. For mitochondria solubilised and washed in LMNG, only GFP and small amounts of Tom40 and Tom22 could be detected (Figure 16A, lane 7). No TIM23 complex protein was co-isolated, indicating that the TIM23 complex dissociated in LMNG. For sample in which digitonin was exchanged to LMNG at the washing step, Tim23 and Tim21 could be co-isolated along with Tom40 and Tom22, but other TIM23 complex components fell apart (Figure 16A, lane 9). Solubilisation and washing with GDN was comparatively more successful. All the tested TOM and TIM23 complex proteins were isolated, although with a lower efficiency compared to digitonin (Figure 16A, lane 8). Exchange of detergent to GDN showed a similar elution profile of the complex as with only digitonin (Figure 16A, lane 10).

As can be seen from this experiment, the solubilisation efficiency of digitonin, GDN and LMNG is similar at $1 \mathrm{wt} \%$. However, LMNG by itself or even after the exchange, is detrimental to the stability of the TIM23 complex. GDN, the synthetic substitute of digitonin, keeps the TIM23 complex intact, and therefore could be a potential new detergent for TIM23 complex structural studies.

A

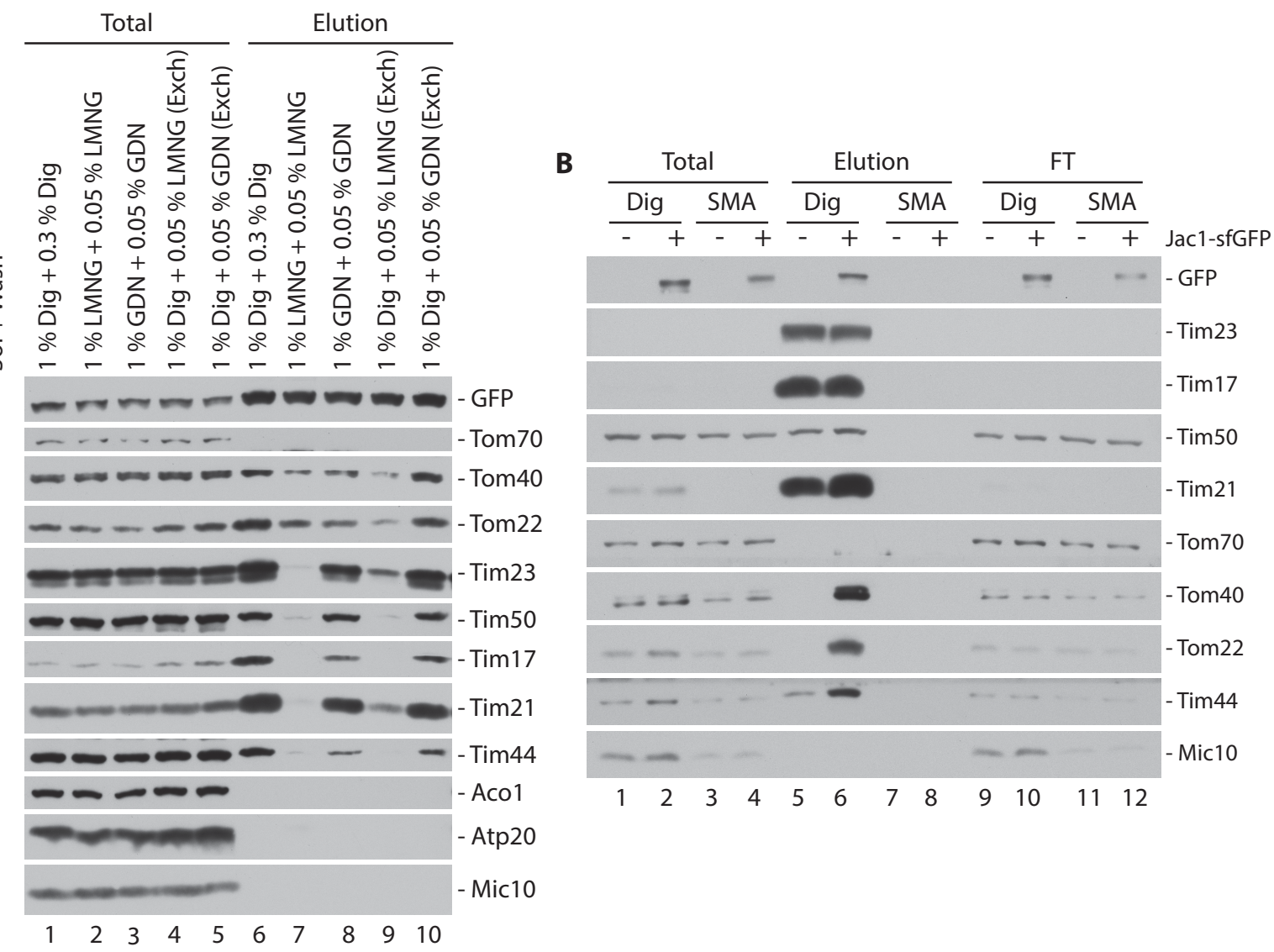

Figure $16 \mathrm{GDN}$ is an alternative detergent that can be used for supercomplex isolation A. The TOM-TIM23 supercomplex was isolated with GFP Nb after import of Jac1-sfGFP as described previously. Mitochondria were solubilised with digitonin, LMNG or GDN. To test the effect of detergent exchange on complex stability, two of the three digitonin solubilised samples 
were washed with LMNG or GDN. Samples were analysed with SDS-PAGE and western blotting. Total $-5 \%$, Elution - 100\%. B. Buffer (-) or Jac1-sfGFP (+) was imported into His-SUMOstar-Tim23 mitochondria. Digitonin or SMA was used for solubilising mitochondria. Isolation was carried out with SUMOstar protease. Samples were analysed by SDS-PAGE and western blotting. Total, FT 1.5\%, Elution - 100\%. Sol: solubilisation, Exch: Exchange, Dig: Digitonin, LMNG: Lauryl Maltose Neopentyl Glycol, GDN: Glyco-diosgenin, SMA: Styrene Maleic Anhydride.

In parallel, another approach involving detergent-free membrane protein extraction was tested. This was performed using styrene-maleic acid copolymers (SMA) (Dörr et al., 2016). With this, membrane proteins can be extracted as polymer-bound nanodiscs, with their native lipid environment intact around them. Import of Jac1-sfGFP into HisSUMOstar-Tim23 mitochondria was carried out. Solubilisation was carried out with either digitonin or SMA, followed by isolation of the TIM23 complex. As could already be seen with the 'total' samples, different proteins were extracted to different levels upon SMA solubilisation. Tom70, Tom40 and Tim50 could be obtained in similar levels to digitonin solubilised mitochondria, whereas other proteins were obtained to a lesser extent (Tom22, Tim44, Mic10) or could not be detected at all (Tim21) (Figure 16B, lanes 2 and 4). After this, it wasn't surprising to not obtain any supercomplex components in the elution fraction (Figure 16B, lane 6 vs lane 8). It could be argued that since the supercomplex would encompass both the outer and inner mitochondrial membrane, the SMA polymer would fail to isolate the supercomplex. However, it also failed to isolate the TIM23 complex by itself, in the absence of Jac1-sfGFP (Figure 16B, lane 5 vs lane 7). Therefore, SMA solubilisation was not considered as a feasible approach.

\subsubsection{Glycerol density gradient separation of the supercomplex}

In order to assess the stability and integrity of the isolated complex, it can be applied to a glycerol density gradient. Previous studies with the TOM and TIM23 complexes by sucrose gradients or size exclusion chromatography have reported that the complexes run as intact units (Stan et al., 2000) (Song et al., 2014) (Ahting et al., 1999) (Denkert et al., 2017). With that in mind, His-Tim23 isolated complexes were analysed utilising a $10-30 \%$ glycerol density gradient. Fractionation was carried out from top of the gradient, so lower molecular weight complexes would be at the top of the gradient and higher molecular weight complexes at the bottom. Three different complexes were purified, as indicated in Figure 17A: (i) the unoccupied TIM23 complex, in the absence of supercomplex-forming protein, (ii) the TIM23 complex in the presence of Jac1-sfGFP and (iii) adding a second step GFP Nb purification to get the TOM-TIM23 supercomplex. For condition (i), no GFP or Tom40 was obtained in the elution fraction, as expected. The presence of Tim23, Tim17 and Tim21 indicated that the TIM23 complex was intact between fractions 2-6 
(Figure 17B). In condition (ii), both GFP and Tom40 are obtained in the elution fraction. They also co-migrated predominantly between fraction 6-9, indicating the formation of a high molecular weight complex between them. Free precursor was also detected at the top of the gradient (Figure 17C). TIM23 complex components showed a partial upshift towards the higher molecular weight fractions, as compared to Figure 17B. This could be the TOM-TIM23 supercomplex. This was confirmed in condition (iii), where the double step purified supercomplex was applied on the gradient. GFP, Tom40, Tim23, Tim17 and Tim21 all migrated predominantly in fraction 6-8, indicating that integrity of the supercomplex is mostly maintained after 2 steps of purification (Figure 17D). However, for all proteins, bands could also be detected in the lower molecular weight fractions, suggesting that the complex is not entirely stable either after the purification or on the gradient.
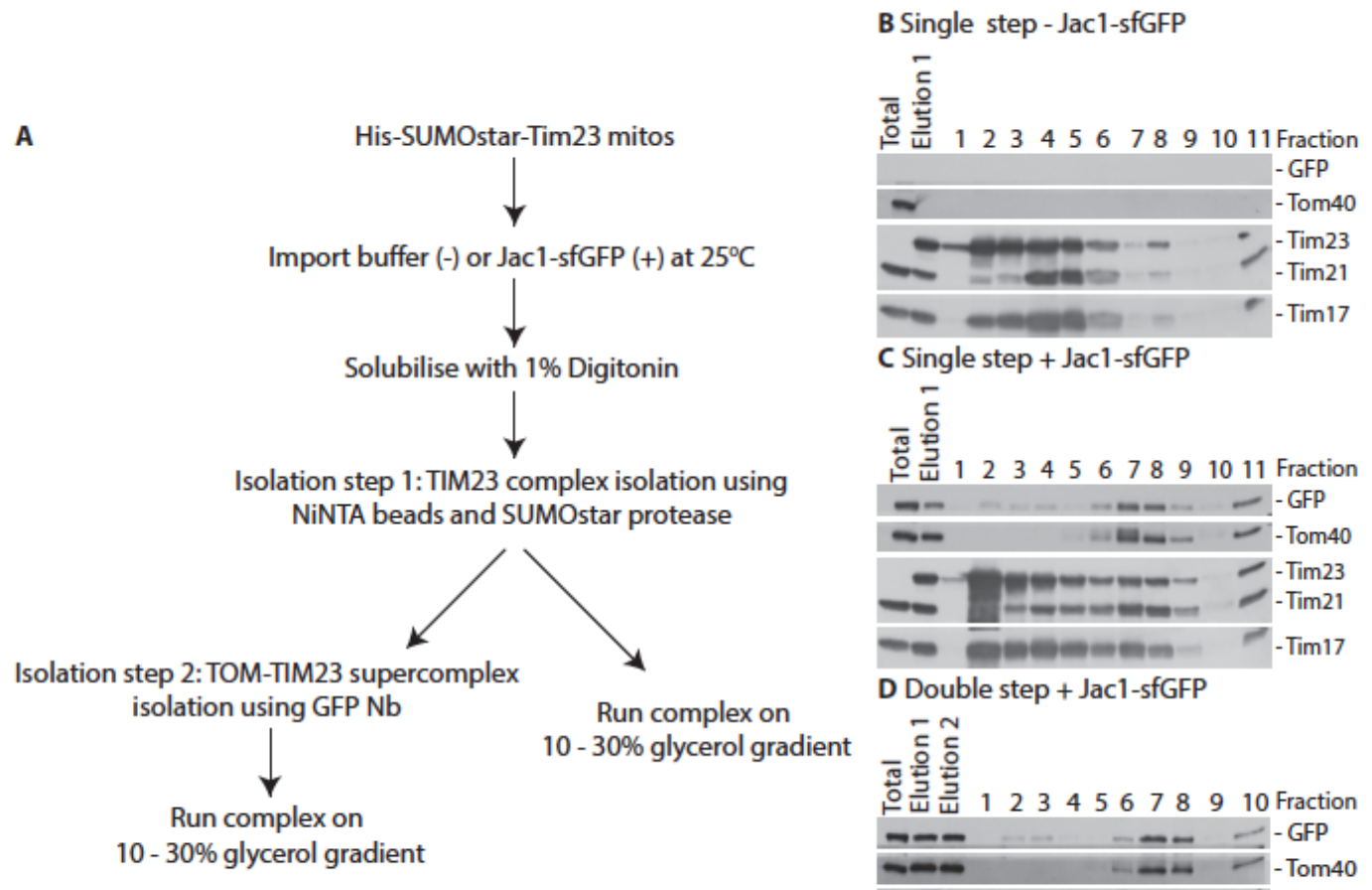

D Double step + Jac1-sfGFP

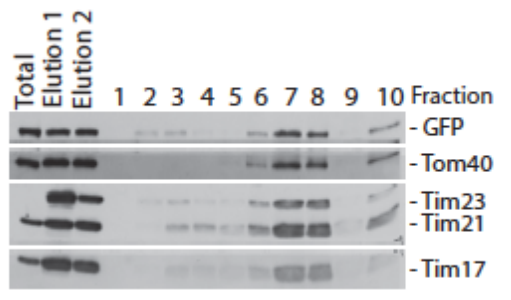

Figure 17 The isolated TIM23 complex and supercomplex on glycerol gradients - A. Scheme for the TIM23 complex isolation - and + Jac1-sfGFP, followed by second step with GFP $\mathrm{Nb}$. Isolated complexes were applied on a glycerol gradient. B. First step TIM23 complex isolated in absence of Jac1-sfGFP was applied on $10-30 \%$ glycerol gradient with $0.3 \%$ digitonin. Centrifugation was performed at $121,262 \times \mathrm{g}$ for $18 \mathrm{~h}$ in SW60Ti rotor at $4^{\circ} \mathrm{C}$. Fractions were collected from the top and were subjected to TCA precipitation. Sample analysis was carried out using SDS-PAGE and western blotting. Total $-0.4 \%$ of the complex loaded on the gradient, Elution $1-1.5 \%$ of the complex loaded on the gradient. C. Same as in B but in the presence of Jac1sfGFP. D. The TOM-TIM23 supercomplex was isolated using a second step with GFP Nb after first step of SUMOstar protease. Gradient conditions remain the same as in B and C. 


\subsubsection{Chemical fixation with glutaraldehyde during the gradient run} (GraFix)

The fragile nature of the TIM23 complex necessitated the introduction of a chemical fixative agent during the gradient centrifugation. This can be achieved by using a glutaraldehyde gradient in parallel to the glycerol one. The complexes are stabilised by gradual formation of cross-links under mild conditions. Such an approach has previously been demonstrated to improve stability and homogeneity for low abundance complexes (Kastner et al., 2008). Glutaraldehyde gradient was optimised to $0-0.05 \%$ and was applied as indicated (Figure 18A). His-SUMOstar-Tim23 mitochondria were used, and the TIM23 complex was isolated after import of Jac1-sfGFP. The resulting eluate was applied on a $10-30 \%$ glycerol $+0-0.05 \%$ glutaraldehyde gradient. After cross-linking with glutaraldehyde, the proteins were not expected to enter an SDS gel, therefore dot-blotting was utilised. As before, the TOM complex (Tom40, Tom22 and Tom20) migrated between fraction 6-9 in the supercomplex form (Figure 18B). TIM23 complex proteins (Tim23, Tim17 and Tim21) and Tim44 migrated both as part of the TIM23 complex (fraction 3-6) and in the supercomplex form (fraction 6-9). Tom70 acted as the negative control since it does not associate with the supercomplex in significant amounts.

More supercomplex can be isolated with the TIM23 complex isolation after import of a supercomplex-forming protein than with the GFP Nb approach directly. With GraFix, different forms of the isolated TIM23 complex could be separated, and the fraction of interest containing the supercomplex can be further analysed. This leads to improved homogeneity, and the more stable complex could also be allowed to adsorb for longer time during grid preparation for EM analysis.

A

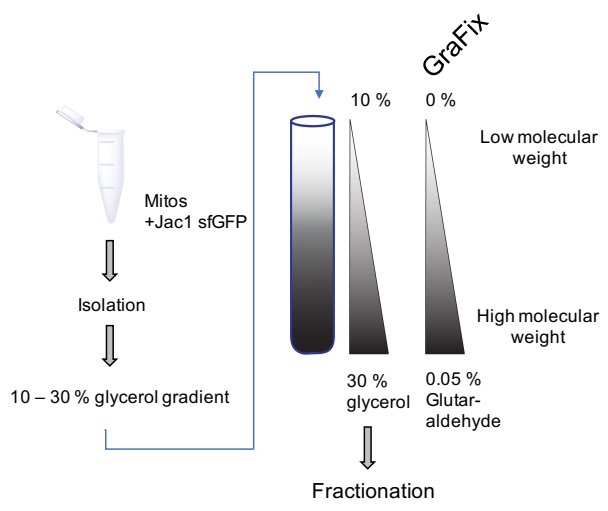

B

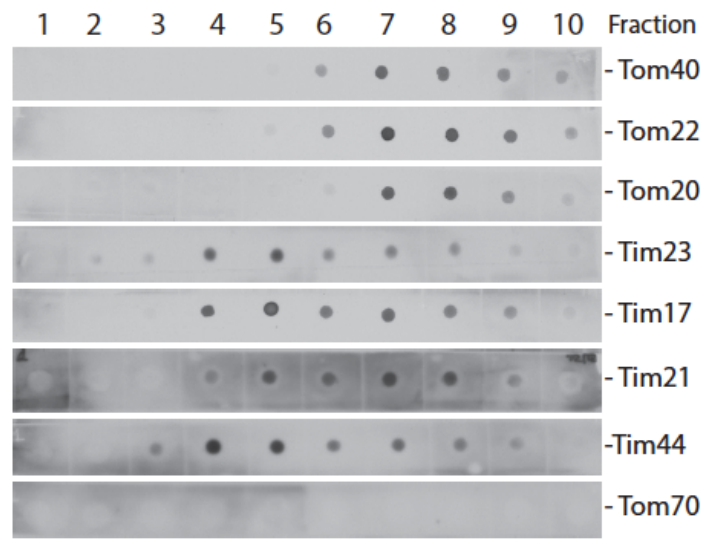

Figure 18 Chemical fixation during gradient centrifugation stabilises the supercomplex - A. Scheme for gradient fixation (GraFix) of the purified TIM23 complex after import of Jac1sfGFP. B. The TIM23 complex was isolated from His-SUMOstar-Tim23 mitochondria after import of Jac1-sfGFP. The complex was separated on a $10-30 \%$ glycerol gradient with $0-0.05 \%$ 
glutaraldehyde. Centrifugation conditions same as described previously. Fractions were collected from top and analysed by dot blotting.

\subsubsection{Detergent exchange during GraFix (GraDeR)}

The TIM23 complex is stable in digitonin and to an extent in GDN. However, previous EM structural analysis showed high levels of digitonin contaminants in the background (Lytovchenko $O$,2012). To overcome this, detergent exchange can be performed. GraDeR or gradient-based detergent removal allows removal of free detergent in a mild manner by applying a reverse gradient of detergent of interest along with the glycerol gradient (Hauer et al., 2015).

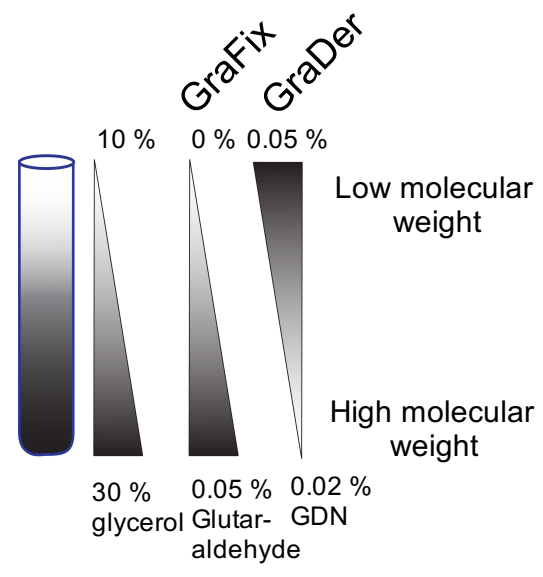

A GraFix

Digitonin purified sample

Gradient: $0.3 \%$ Digitonin, 10-30\% glycerol, $0-0.05 \%$ glutaraldehyde

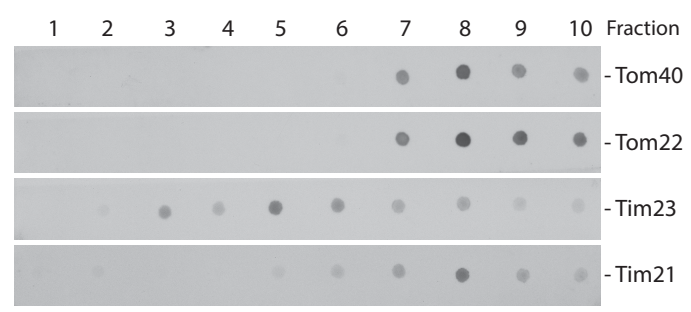

B GraDeR + GraFix Digitonin purified sample Gradient: 0.05-0.02\% GDN, 10-30\% glycerol, 0 - 0.05\% glutaraldehyde

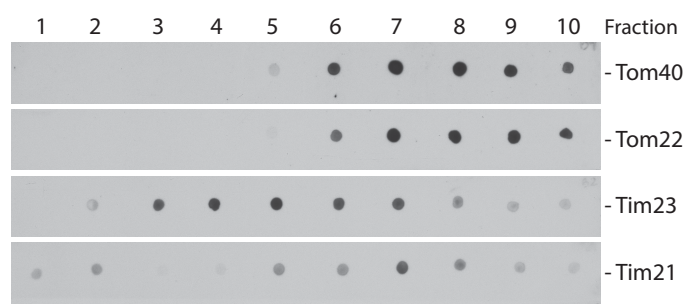

Figure 19 Detergent exchange during gradient centrifugation stabilises the supercomplex - The TIM23 complex isolated from His-SUMOstar-Tim23 mitochondria in presence of Jac1-sfGFP. The complex was extracted using digitonin, and elution fraction was loaded on a $10-30 \%$ glycerol, $0-0.05 \%$ glutaraldehyde gradient A. without GraDeR $(0.3 \%$ digitonin in gradient) or B. with GraDeR $(0.05-0.02 \%$ GDN). Centrifugation conditions same as described previously. Fractions were collected from the top and analysed by dot blotting.

The TIM23 complex was purified from His-SUMOstar-Tim23 mitochondria following import of Jac1-sfGFP. This was applied on a glycerol-glutaraldehyde gradient, with or without GraDeR. With only GraFix, when the sample was in digitonin, the supercomplex migrated between fraction 7-9 (Figure 19A). On addition of an inverse $0.05-0.02 \%$ GDN gradient, there was a slight upshift in the supercomplex migrating pattern, such that it was 
predominantly present between fraction 6-9 (Figure 19B). The exchange of detergent most likely affected the complex sedimentation rate, leading to the upshift.

\subsubsection{Structural analysis of the isolated TIM23 complex and the TOM-TIM23 supercomplex}

\subsubsection{Negative stain EM analysis of the supercomplex}

Structural analysis of mitochondrial translocases remains an ongoing challenge. Recently, advances have been made in structural information of the TOM complex (Shiota et al., 2015) (Bausewein et al., 2017). The small size and dynamic nature of the TIM23 complex, however, makes it a challenging study. After optimising the condition for purifying the TIM23 complex and the TOM-TIM23 supercomplex in quantifiable amounts, negative stain EM analysis was performed in collaboration with Dr. Niels Fischer from the group of Prof. Dr. Holger Stark (Department of Structural Dynamics, Göttingen).

A double step TOM-TM23 supercomplex isolation, as optimised in section 3.1.2.1 (Figure 15B lane 5), showed the presence of a large number of particles after negative staining (Figure 20B). The corresponding control sample, which did not have any TIM23 complex, did not show any particles (Figure 20A). Since the preparation was highly heterogeneous in terms of complex shape and size, it was decided to improve the complex stability with TIM23 complex isolation after Jac1-sfGFP import followed by GraFix. Based on previous fixation results (Figure 18B), fraction 7 was decided to be used for analysis. In the absence of glutaraldehyde (Figure 20C), the particles seen are small and abundant. On applying GraFix (Figure 20D), several larger particles of similar shape and size were obtained (blue circles). Also present were digitonin micelles (white arrowheads). To eliminate the background contamination from detergent, GraDeR was applied to exchange the detergent from digitonin to GDN. Fraction 6-8 were pooled and concentrated with $100 \mathrm{kDa}$ cut-off centrifugal concentrator columns. The supernatant was used for sample preparation, as it was expected to have enriched supercomplex, while the detergent micelles would flow through. Here, the buffer control showed micelles of various sizes (Figure 20E), indicating that some micelles greater than $100 \mathrm{kDa}$ survived the concentration step. The protein sample revealed a mixture of micelles and particles (Figure 20F). Detergent background from the previous step was gone, suggesting that GraDeR worked. However, the gradient step was diluting the original sample amount by almost 10 -fold, resulting in low particle abundance sample. Therefore, single particle analysis could not be performed. Further optimisations are required to improve the sample yield and homogeneity. 


\section{Results}

A

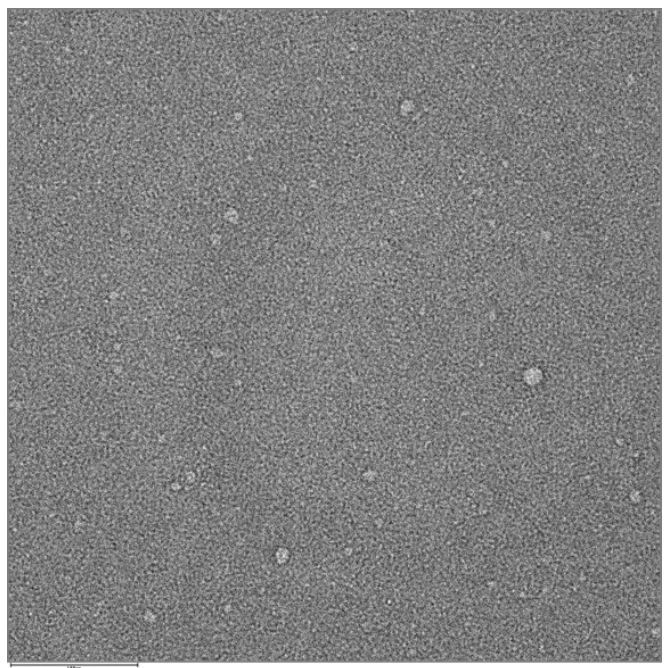

C

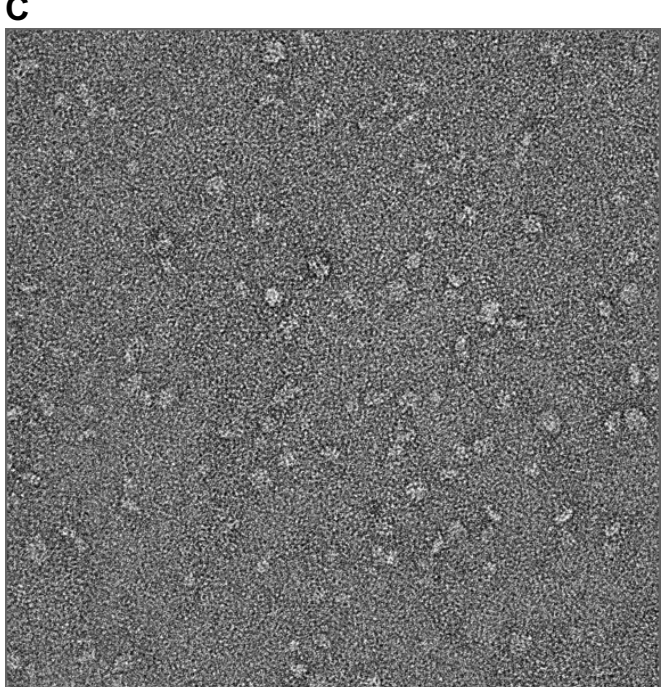

E

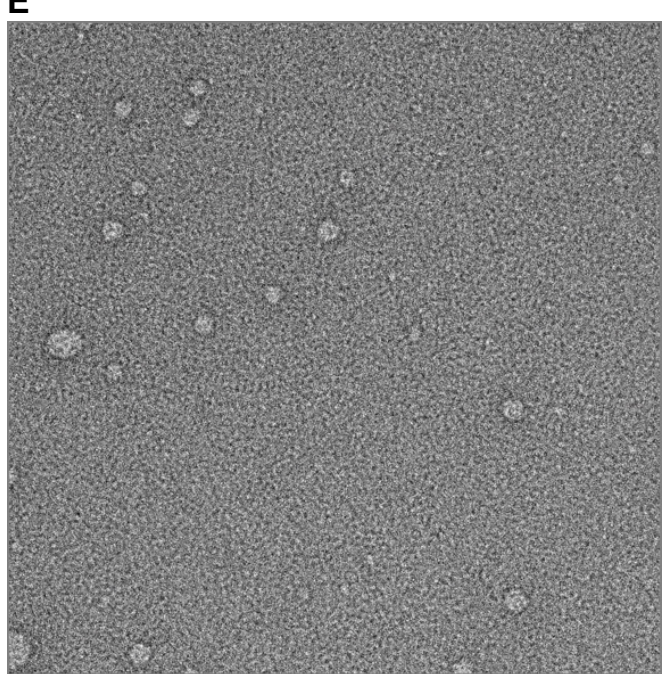

B

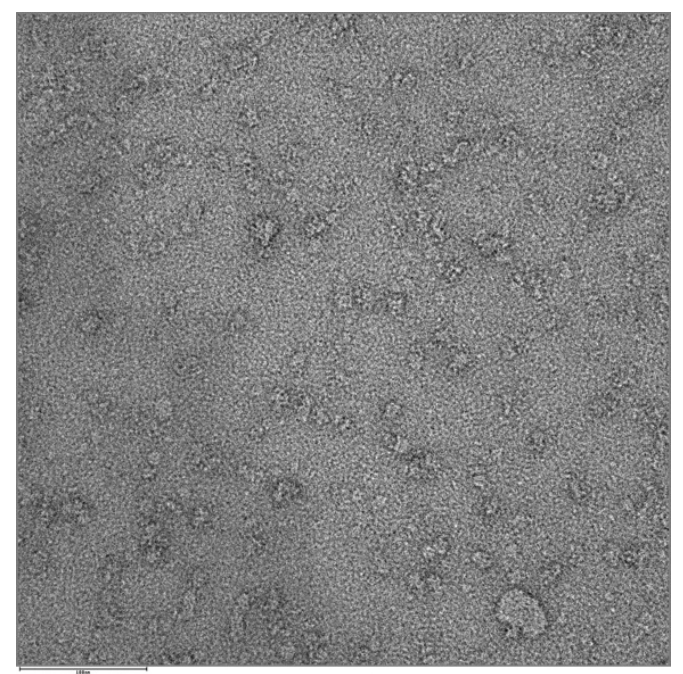

D

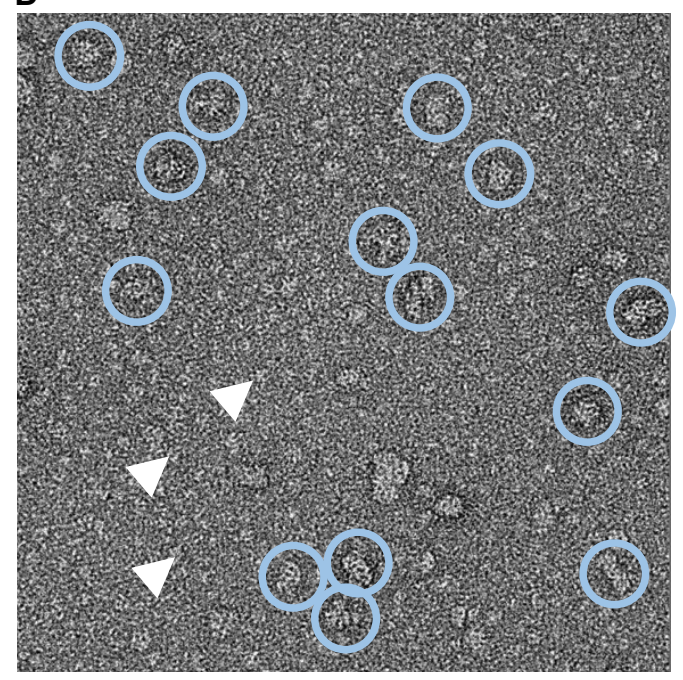

$\mathbf{F}$

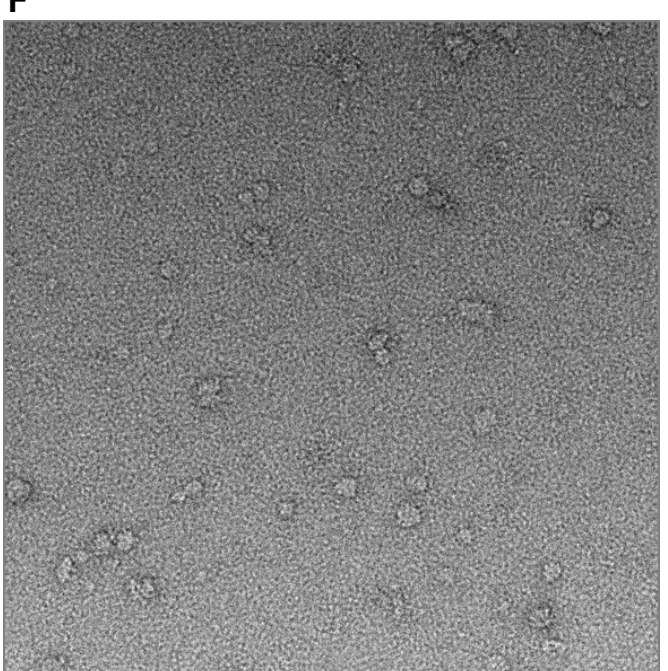

Figure 20 Representative negative stain images - A-B. Micrographs of (B) 2 step TOM-TIM23 supercomplex purification and (A) buffer control. C-D. Micrographs of Fraction 7 after gradient centrifugation of the TIM23 complex purified after Jac1-sfGFP import (C) without and (D) with GraFix. Blue circle: particles, white arrowheads: detergent micelles. E-F. Micrographs of (F) concentrated fraction 6-8 from GraFix and GraDeR of the TIM23 complex purified after Jac1-sfGFP import and (E) corresponding buffer control. (Scale bar: $100 \mathrm{~nm}$ ) 


\subsubsection{Cross-linking mass spectrometry analysis to define protein dynamics in the TIM23 complex and the TOM-TIM23 supercomplex}

An alternative approach to obtain structural information utilises chemical cross-linking of the isolated complex followed by mass spectrometry. Both the unoccupied TIM23 complex as well as the TOM-TIM23 supercomplex can be subjected to cross-linking to obtain an insight into the interactions and spatial orientations of subunits with respect to each other. To follow this approach, the TOM-TIM23 supercomplex was isolated. Firstly, several chemical cross-linkers were tested for the selection of a cross-linker for further experiments. The cross-linkers that were tested were homo-bifunctional amino group reactive molecules, being either membrane permeable (DSS and DSG) or membrane impermeable (BS2G and BS3). Higher cross-linked adducts could be observed for Tim23, Tim21, Tom22 and Tom20, compared to the sample without cross-linker (Figure 21, lane 1-24). For Tim44 and Tim50, the higher cross-links were most likely too big to even enter the SDS gel (Figure 21, lane 25-36). Since the cross-linking pattern of the cross-linkers was similar, DSS was selected for its ability to permeate the membrane, as this property can be utilised for an in organello cross-linking approach in the future.

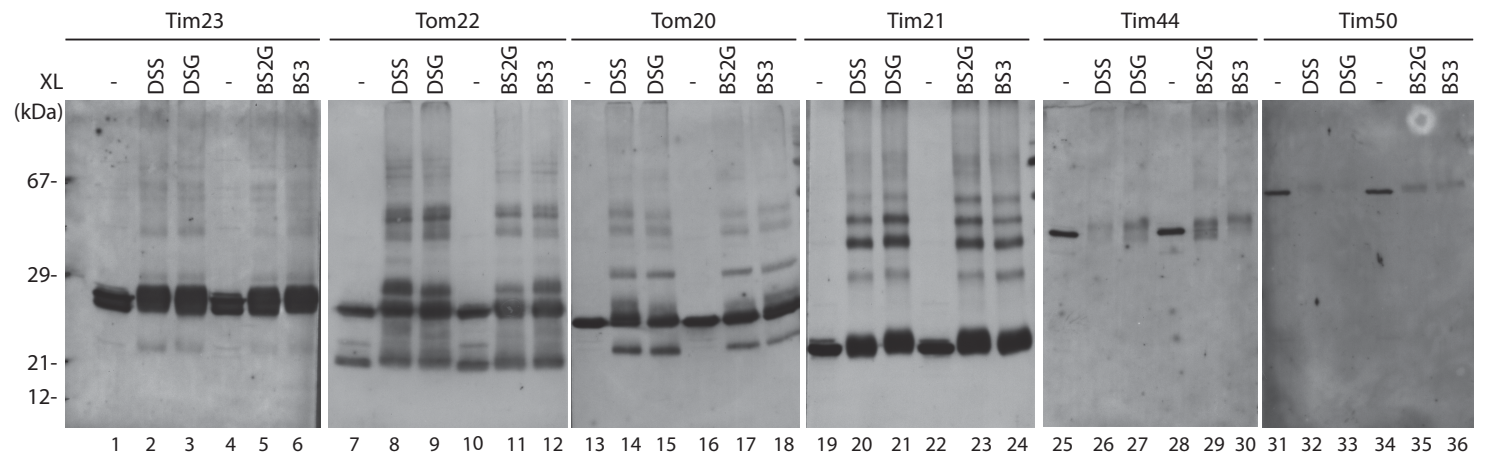

Figure 21 Comparison of different cross-linkers on the isolated TOM-TIM23 supercomplex - A two step supercomplex isolation was carried out from His-SUMOstar-Tim23 mitochondria subjected to Jac1-sfGFP import. Isolation was performed using SUMOstar protease and GFP Nb as described previously. Isolated complex was treated with $500 \mu \mathrm{M}$ of different crosslinkers for $30 \mathrm{~min}$ on ice. Reaction was quenched with $250 \mu \mathrm{M}$ Glycine $\mathrm{pH} 8.0$ for additional 15 mins on ice. Samples were analysed using SDS-PAGE and western blotting. XL: cross-linker, DSS: disuccinimidyl suberate, DSG: disuccinimidyl gluterate, BS2G: bissulfosuccinimidyl glutarate, BS3: bissulfosuccinimidyl suberate.

A comparison of cross-links in the TIM23 complex isolated in the absence or presence of Jac1-sfGFP would be helpful in monitoring the dynamics of individual proteins, as well as to see any structural rearrangements which might be happening. To that end, the TIM23 complex was purified on a large scale in the absence or presence of Jac1-sfGFP. Crosslinking was carried out with DSS, followed by quenching with glycine. Mass spectrometry analysis was performed by Andreas Linden from the group of Prof. Dr. Henning Urlaub (Bioanalytical Mass Spectrometry, Max Planck Institute for Biophysical Chemistry, 
Göttingen). The PSM (peptide spectrum matches) results for both samples were analysed. The PSM value of a protein indicates the total number of identified peptide sequences obtained for that protein after mass spectrometry. Fold change for individual proteins upon addition of Jac1-sfGFP was calculated based on their PSM values. Since the TIM22 complex is known to not co-purify with the TIM23 complex, it was selected as a negative control. Indeed, the TIM22 complex proteins (Tim8, Tim13, Tim10, Tim54 and Tim22) showed no change in their PSM values between the two samples (Figure 22). PSM values of bait protein Tim23 remained the same. In addition, a slight increase was observed for Tim21, Mgr2, as well as PAM complex proteins Hsp70 and Tim44 when Jac1-sfGFP was present. Not surprisingly, the biggest fold change was observed for the TOM complex. Tom40 and Tom22 showed a 9-10 fold increase, whereas Tom20 and Tom5 showed a 7-8 fold increased PSM number. Surprisingly, Tom70 and Tom71 also had increased PSM values, even though they could never be detected by western blotting to be a part of the supercomplex. The difference in fold change for subunits of the TOM complex in the presence of Jac1-sfGFP could be due to: (i) different stoichiometry of the protein within the isolated complex, or (ii) the number of peptides of that protein which were identified, since all proteins were covered to a different extent.

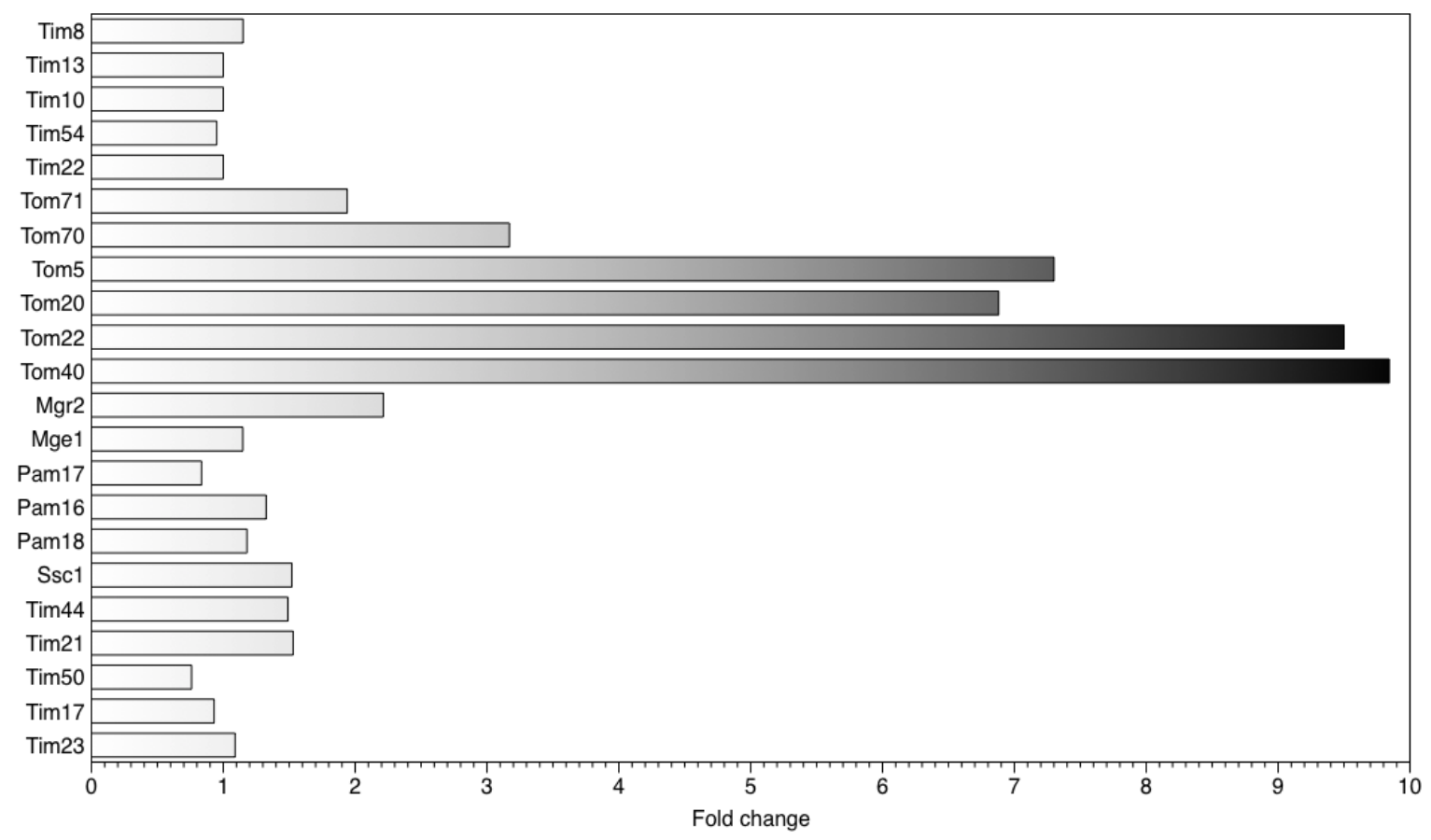

Figure 22 Fold change in peptide spectrum matches of TOM, TIM23 and TIM22 complex components - Jac1-sfGFP was imported or not into His-SUMOstar-Tim23 mitochondria and the TIM23 complex isolation was carried out for both samples. These were subjected to mass spectrometry analysis after DSS cross-linking. Peptide spectrum matches obtained for both were used to calculate the fold change for different proteins between the samples. 
Furthermore, the cross-links obtained were analysed based on the known structural information of individual subunits. An overview of the non-redundant cross-links identified by mass spectrometry is shown (Figure 23A,B and C).
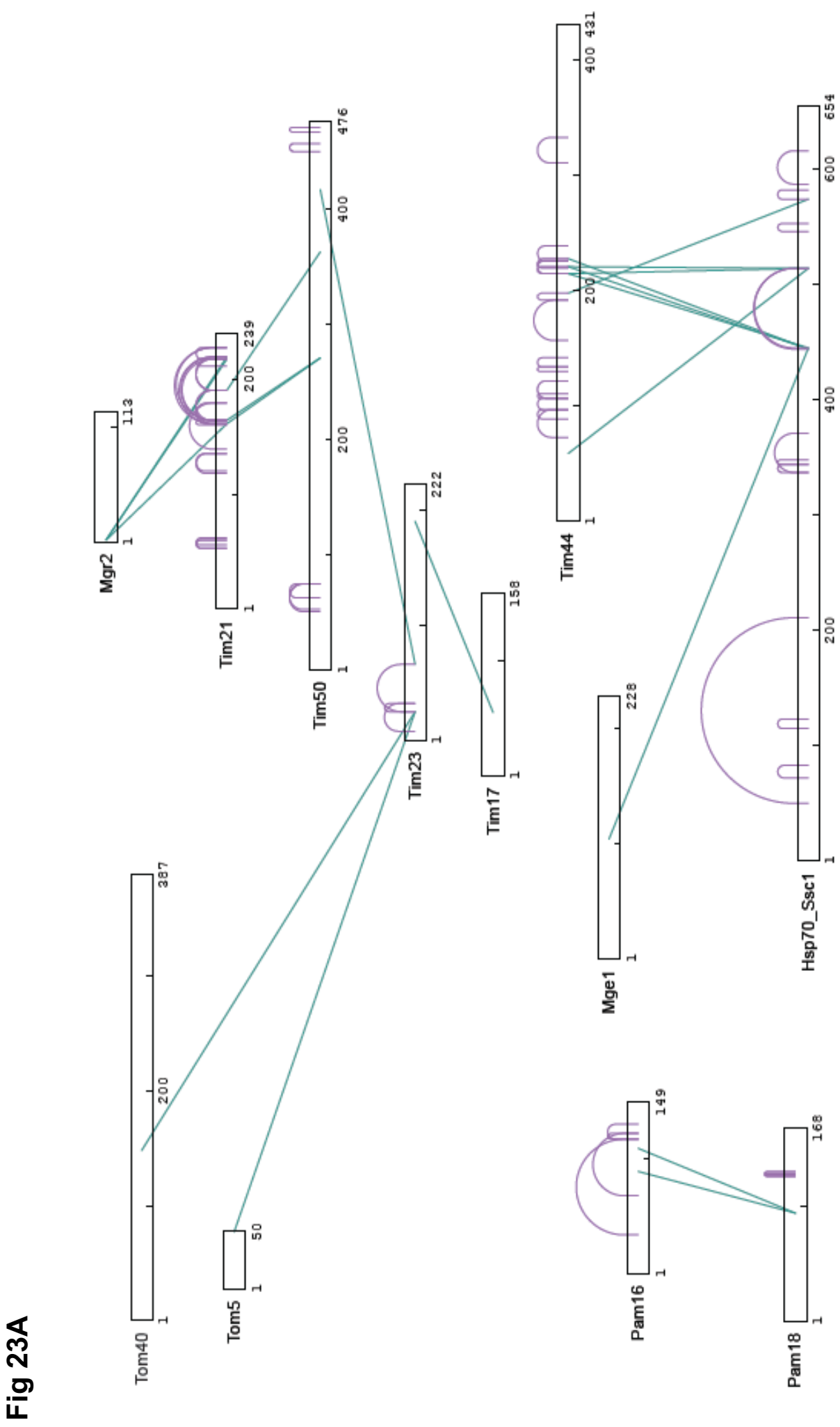


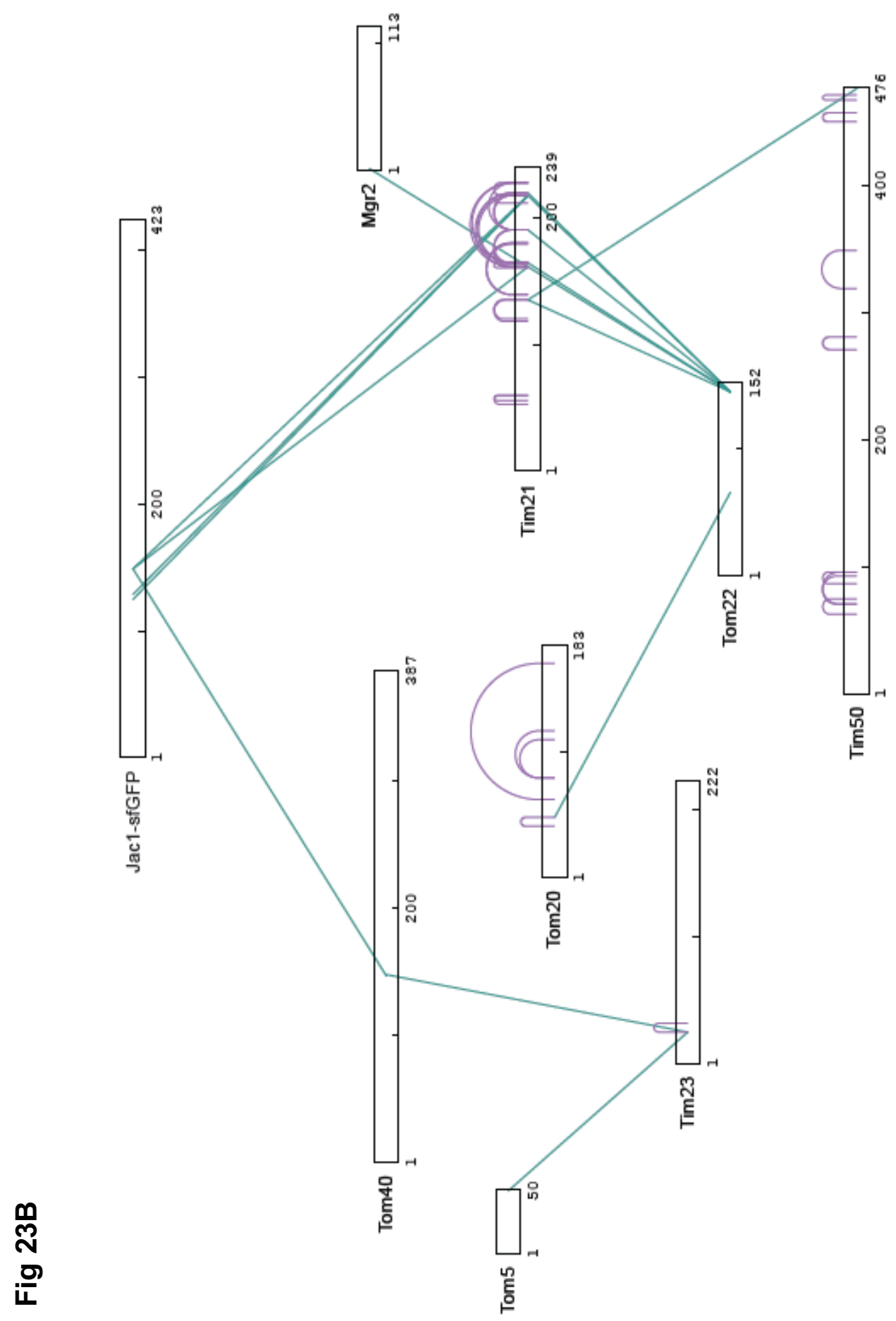

In the absence of Jac1-sfGFP, cross-links were majorly observed within the subunits of the TIM23 complex and the PAM complex (Figure 23A). For the TIM23 complex, interprotein links were detected between Tim23 (K66)-Tim50 (K417), Tim23 (K190)-Tim17 (K55), Tim50 (K363, K217)-Tim21 (K190, K161, K164) and Tim21 (K161, K217, K219)Mgr2 (K2). 


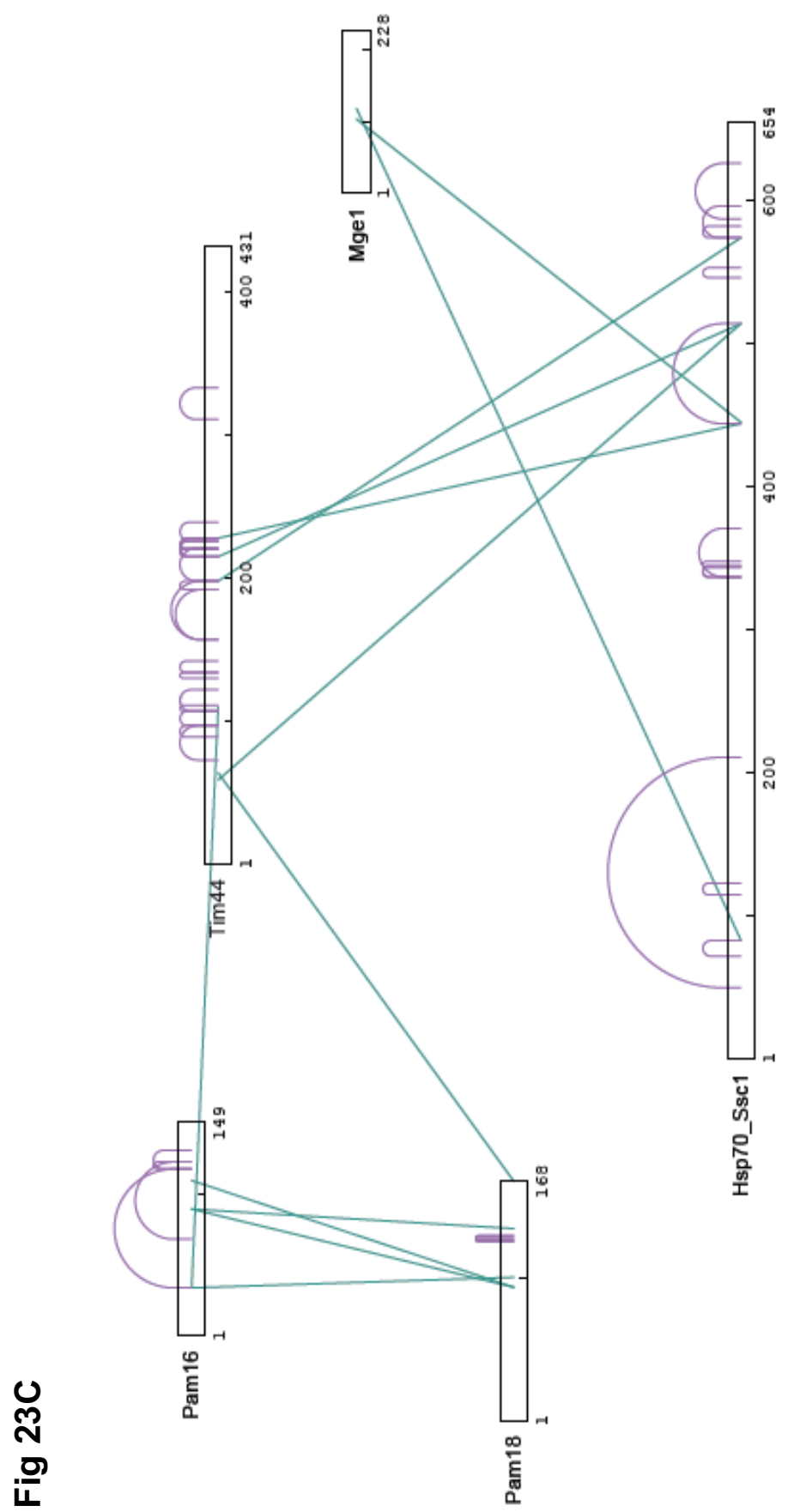

Figure 23 Visualisation of cross-links obtained in the absence and presence of Jac1sfGFP - The TIM23 complex was isolated in (A) absence or (B and C) presence of Jac1-sfGFP. Cross-linking was carried out with $1 \mathrm{mM}$ DSS for $3 \mathrm{~h}$ on ice. The reaction was quenched with 250 $\mu \mathrm{M}$ Glycine $\mathrm{pH} 8.0$ for additional 30 mins on ice. Samples were analysed by mass spectrometry. Green: Inter-protein cross-link, Purple: intra-protein cross-link.

All cross-links were observed for the IMS- or matrix-exposed domains of these proteins. Tim23 $3^{\mathrm{IMS}}-\mathrm{Tim}_{50} 0^{\mathrm{IMS}}$ and $\mathrm{Tim} 0^{\mathrm{IMS}}-\mathrm{Tim} 1^{\mathrm{IMS}}$ interactions have previously been reported (Alder et al., 2008) (Tamura et al., 2009) (Lytovchenko et al., 2013) (Bajaj et al., 2014a). Surprisingly, an interaction was detected between K66 of Tim23 and K417 of Tim50 in this study. K417 forms a part of the presequence binding domain in Tim50, whose structure is 
not known. Therefore, the cross-link indicated the proximity of Tim $23^{\text {IMS }}$ and Tim50 $0^{\text {PBD }}$ in the absence of a protein being imported. Additionally, Tim21 interaction with Tim50 was occurring in the Tim50 core domain. Tim23 loop 3 (between TM3 and TM4) in the matrix was linked to Tim17 loop 1(between TM1 and TM2). Lastly, the N-terminus of Mgr2 (K2) was identified to form a cross-link with Tim2 $1^{\mathrm{IMS}}$. This finding was supported by the known role of Mgr2 in Tim21 recruitment to the TIM23 ${ }^{\text {CORE }}$ complex (Gebert et al., 2012). The Nterminus of Tim23 ${ }^{\mathrm{IMS}}$ (K25) also formed cross-links with the predicted IMS-exposed domain of Tom40 (K148) and the known IMS-exposed domain of Tom5 (K50), pointing towards proximity between the extreme $\mathrm{N}$-terminus of Tim23 $3^{\mathrm{IMS}}$ and the trans side of the TOM complex. Taken together, these confirm a compact TIM23 complex structure with subunits arranged in close apposition.

In the PAM complex, cross-links were observed between Pam16 (K89, K109)-Pam18 (K94), Tim44 (K228, K198, K215, K221, K59)-Ssc1(K444, K574, K514, K445) and Ssc1 (K445)-Mge1 (K104). Pam16 and Pam18 were interacting through their J-like and Jdomains respectively. These domains have been reported to form a heterodimeric complex (Mokranjac et al., 2006) for regulating the Pam18 function. Additionally, crosslinks were observed between Tim44 N-terminus - C-terminus interface and Ssc1 SBD. Furthermore, Ssc1 was also found to interact with Mge1, its nucleotide exchange factor in the matrix.

Intra-protein cross-links were also acquired, predominantly for Ssc1, Tim44, Pam16, Pam18, Tim21 ${ }^{\mathrm{IMS}}, \mathrm{Tim}_{50}$ and Tim23 ${ }^{\mathrm{IMS}}$. Ssc1 intra-protein cross-links were observed between Lys residues of the same domain, i.e., cross-linking was taking place within the NBD (nucleotide binding domain) or the SBD (substrate binding domain). This suggested that either these domains formed structurally distinct units or the Lys residues were not accessible for cross-linking. For Tim44, NTD (N-terminal domain) residues formed more cross-links than CTD (C-terminal domain) residues. This could be due to the intrinsically disordered nature of the NTD, which would promote the probability of interactions compared to the structured CTD. On mapping the Tim $21^{\mathrm{IMS}}$ cross-links on the crystal structure (PDB ID: 2CIU, (Albrecht et al., 2006)), most were found to be within the range of DSS spacer length $(<15 \AA)$. A few links $(211-217,160-164,219-161)$ demonstrated higher length between the corresponding amino acids. This could be due to the fact that the crystal structure represents a recombinantly purified protein, whereas the current study deals with an in organello purified complex, and could therefore have different conformation and flexibility of proteins. Additionally, cross-links were obtained within the $\mathrm{N}$-terminus Tim23 ${ }^{\mathrm{IMS}}$ domain, potentially due to dimerisation of Tim23.

For the TIM23 complex isolated after import of Jac1-sfGFP, inter- and intra-protein crosslinks were acquired for the TOM, TIM23 and PAM complexes (Figure 23B and C). Among 
the TIM23 complex subunits, Mgr2 (K2)-Tim21 (K161) and Tim21 (K135)-Tim50 (K476) retained their cross-linking, although at a lower extent compared to the sample without Jac1-sfGFP (Figure 24).

A

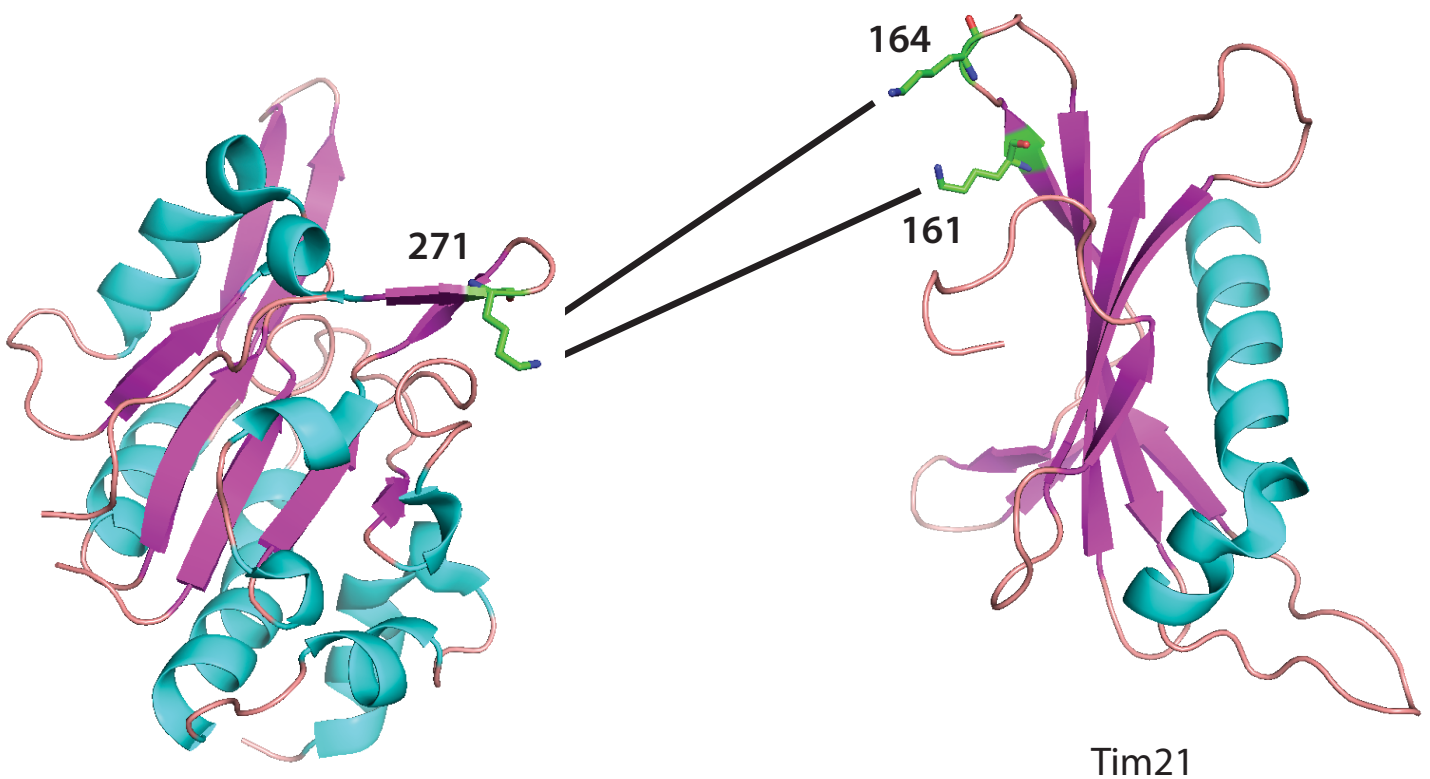

$\operatorname{Tim} 50$

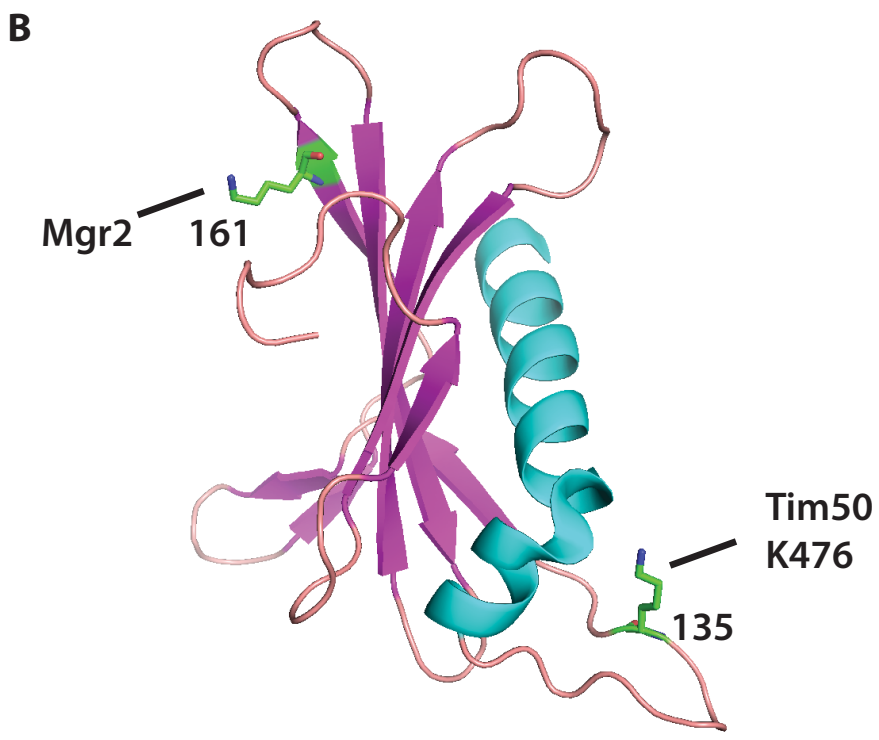

Figure 24 Tim50-Tim21 cross-links in the absence and presence of Jac1-sfGFP - Crosslinking sites between Tim2 $1^{\mathrm{IMS}}$ and Tim50 $0^{\mathrm{IMS}}$ in the (A) absence and (B) presence of Jac1-sfGFP are shown on their cartoon representation. Tim21 (PDB ID: 2CIU (Albrecht et al., 2006)), Tim50 (PDB ID: 3QLE (Qian et al., 2011)). Cross-linked Lys residues are indicated in green. Cyan: $\alpha-$ helix, magenta: $\beta$-sheet.

Since Tim21 engages with Tom22 during supercomplex formation, it could have less interaction sites for Tim50 and Mgr2. These findings were supported by previously demonstrated Tim21 dissociation from Tim50 in the presence of a presequence 
(Lytovchenko et al., 2013). Also, differently localised residues of Tim21 were found to interact with Tim50 in the absence or presence of Jac1-sfGFP. K161 and K164, between $\beta 4$ and $\beta 5$, were in the vicinity of Tim50 in the absence of Jac1-sfGFP (Figure 24A), while K135 between $\alpha 2$ and $\beta 1$ interacted with Tim50 in the presence of Jac1-sfGFP (Figure 24B). This suggested that Tim $21^{\text {IMS }}$ undergoes a conformational rearrangement during the formation of a supercomplex.

A

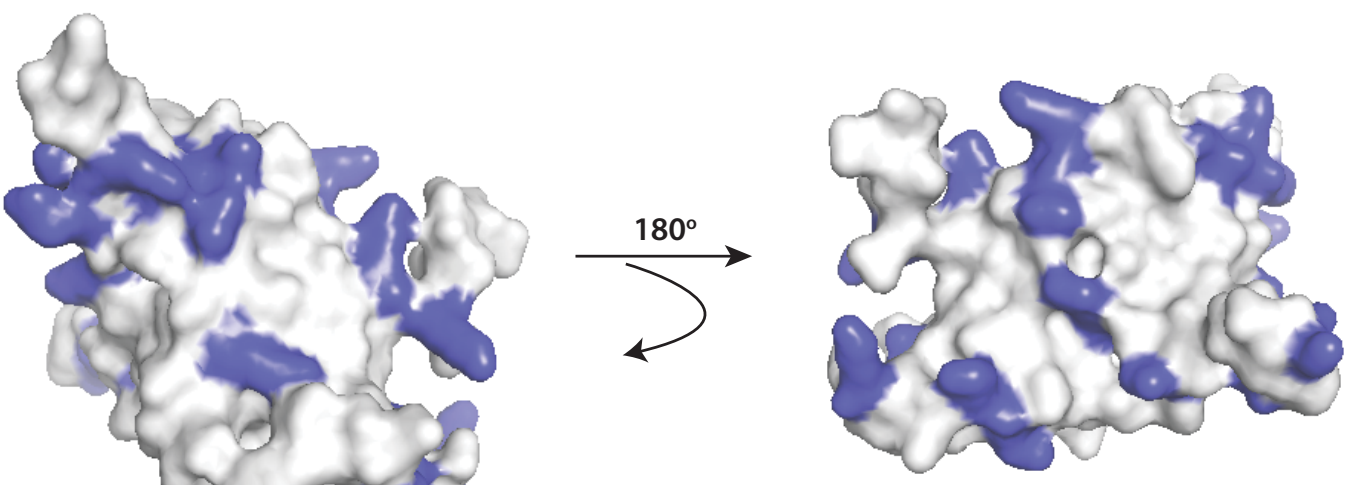

B
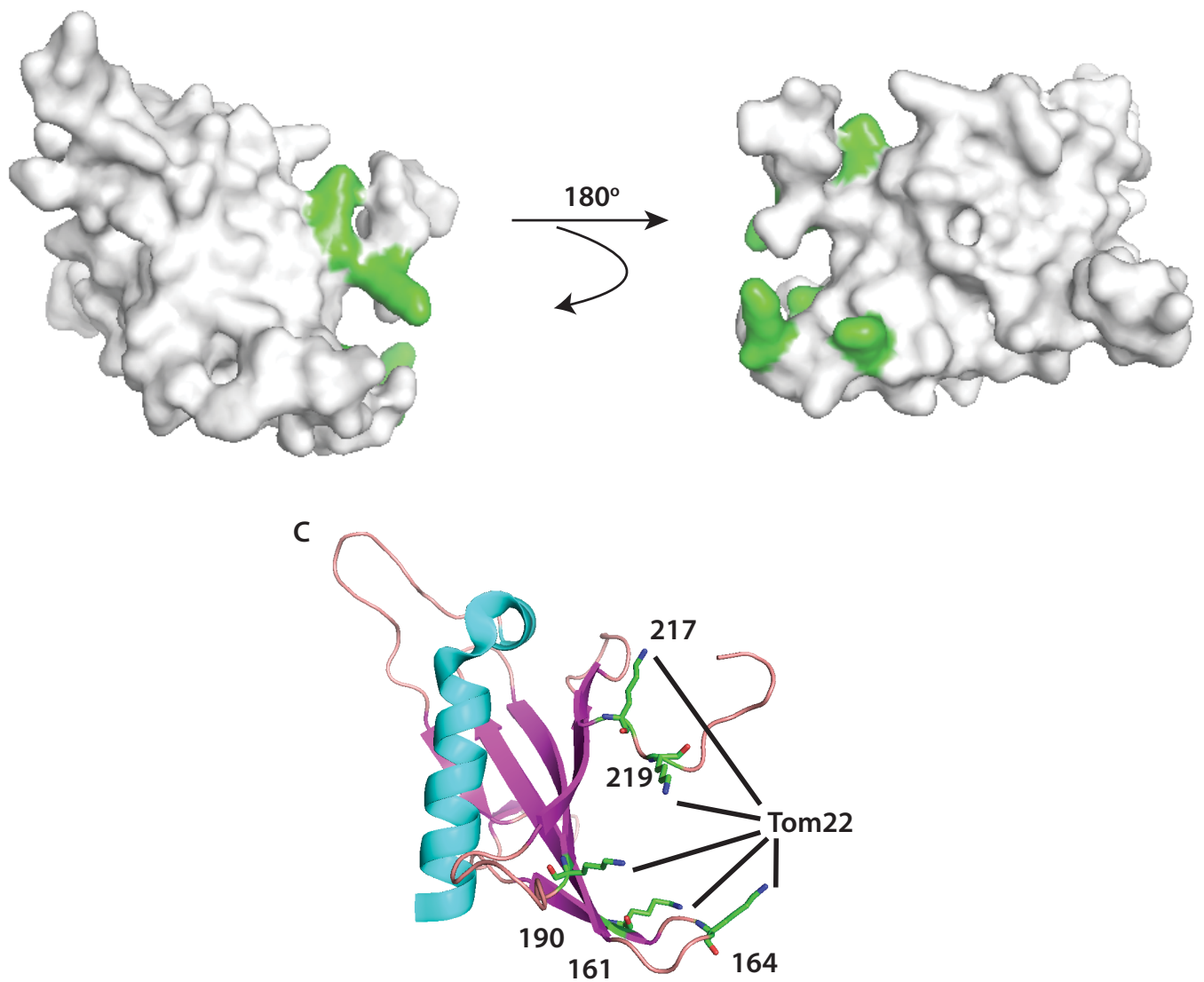

Figure 25 Tim21 cross-links with Tom22 in the presence of Jac1-sfGFP - (A) and (B) Surface representation of Tim2 $1^{\text {IMS }}$ indicating its positively charged (blue) and Tom22-interacting (green) regions. (C) Tim21 $1^{\mathrm{MS}}$ cartoon representation of individual Tom22-interacting Lys residues. Cyan: $\alpha$-helix, magenta: $\beta$-sheet. 
Tim21 interaction with Tom22 in the IMS has previously been reported (Chacinska et al., 2005). Negatively charged residues on the IMS-exposed domain of Tom22 were shown to be interacting with positively charged residues on Tim2 $1^{\text {IMS }}$ surface (Albrecht et al., 2006). Indeed, an overlap could be observed between the positively charged residues and Tom22- interacting residues when they were mapped on the Tim2 $1^{\text {IMS }}$ structure (Figure $25 A$ and $B$ ). These residues seemed to localise around a pocket formed by the $\beta$-sheets and loops of Tim2 $21^{\mathrm{IMS}}$, specifically the loop between $\beta 4$ and $\beta 5$, C-terminus of $\beta 6$ and Cterminal loop after $\beta 8$ (Figure 25C). Furthermore, Tim23 (K25) also retained its interactions with Tom40 (K148) and Tom5 (K50). Since these interactions could be observed independent of the presence of a presequence-containing protein, the $\mathrm{N}$ terminus of Tim23 could be always in the vicinity of the TOM complex under physiological conditions. In the TOM complex, inter-protein cross-links were obtained between Tom20 (48) and Tom22 (K66) on the cytosolic side. This finding is supported by the interactions observed between the cytosolic domains of these proteins (Shiota et al., 2011).

A

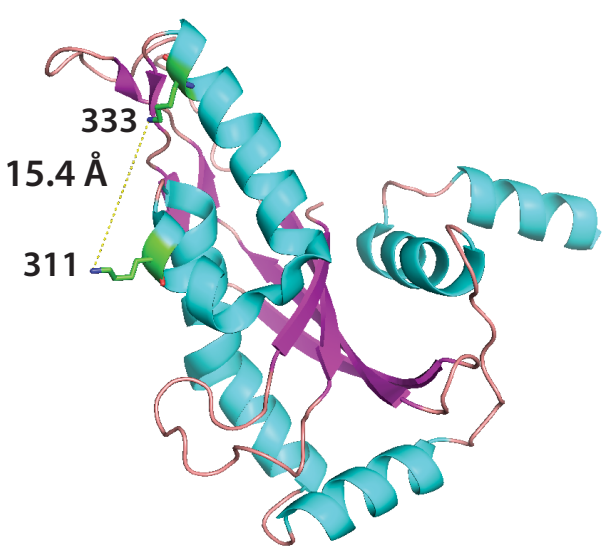

B

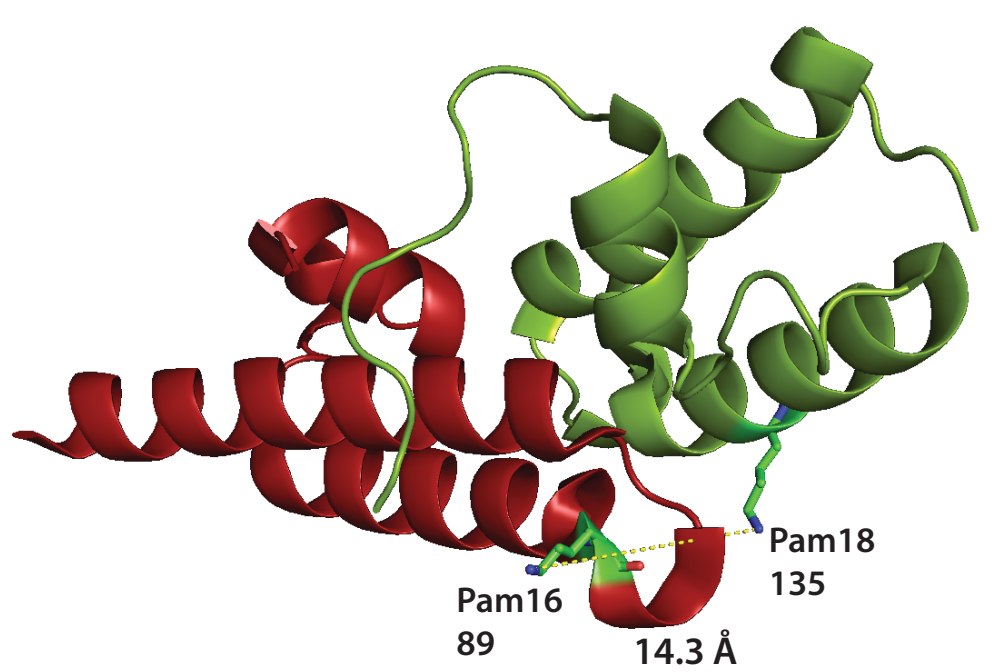

Figure 26 PAM complex subunits undergo intra- and inter-protein cross-linking - (A) Cartoon representation of Tim44 CTD (PDB ID: 2FXT, (Josyula et al., 2006)) indicating its intra- 
protein cross-link. (B) Cartoon representation of the Pam16-Pam18 heterodimer (PDB ID: 2GUZ, (Mokranjac et al., 2006)) indicating the inter-protein cross-link. Cyan: $\alpha$-helix, magenta: $\beta$-sheet, green: Pam18, red: Pam16. Distance between two cross-linked residues is indicated by a dotted yellow line.

The PAM complex cross-links in the presence of Jac1-sfGFP hinted at a slightly altered arrangement within the subunits (Figure 23C). Ssc1-Tim44 and Ssc1-Mge1 interactions were not changed drastically compared to the sample without Jac1-sfGFP. However, cross-links were obtained between Pam16 (K34)-Tim44 (K110) and Pam18 (K164)-Tim44 (K64). Also, Pam16-Pam18 interactions increased in the presence of Jac1-sfGFP, as indicated by the more non-redundant cross-links observed between the heterodimer. The only observed cross-link which could be mapped on the structure of the Pam16-Pam18 heterodimer demonstrated the vicinity of the two residues (Figure 26B). Taken together, these results suggested that structural rearrangement was taking place within the PAM complex subunits as a part of the TOM-TIM23 supercomplex.

Intra-protein cross-links in the presence of Jac1-sfGFP remained by and large similar to the sample without Jac1-sfGFP, except for a couple of additional cross-links within Tim50 IMS , Ssc1 and Tim44. Again, the Tim44 intra-links were predominantly in the NTD. The only CTD cross-link obtained (K311-K333) was mapped on the known Tim44 CTD crystal structure (Figure 26A). Another interesting finding was the almost complete lack of intra-protein links for Tim23 $3^{\mathrm{IMS}}$. This, however, was not surprising since the Tim23 dimer is known to dissociate in the presence of a presequence (Bauer et al., 1996) (Alder et al., 2008). Tim17 (K141) and Ssc1 (K152) also showed cross-linking to themselves (a peptide containing K141 was linked to another peptide containing K141), suggesting that they could potentially be present as a multimer within their respective complexes.

Taken together, these findings provide structural insight into the organisation and dynamics of TOM, TIM23 and PAM complex subunits in their free state and in a substrateoccupied supercomplex state. Some of the cross-links reported here are supported by previously demonstrated protein interaction data. Additionally, a new understanding was obtained regarding interactions and proximity between proteins whose structural information is not known so far. Furthermore, rearrangement in the presence of a translocating precursor protein could also be observed for subunits of the TIM23 and PAM complex.

\subsection{Expansion of substrate spectrum of the TIM22 complex}

The TIM22 complex or the carrier translocase has mainly been characterised in yeast. Known substrates of this complex lack a predicted presequence at the $\mathrm{N}$-terminus and 
contain multiple internal targeting signals in the mature protein. However, so far, knowledge on the substrate spectrum of TIM22 is very limited. Known substrates include the six transmembrane-spanning metabolite carriers, AAC (ADP/ATP carrier), PiC (Phosphate carrier) and DiC (Dicarboxylate carrier), as well as the four transmembranespanning inner membrane translocase subunits, Tim23, Tim17 and Tim22. To broaden the substrate repertoire, a quantitative proteomics approach was utilised, using dimethyl labelling of proteins to compare a Tim22 mutant mitochondria with the control WT.

\subsubsection{Characterisation of the Tim22 temperature sensitive strain}

\subsubsection{Selection of Tim22 temperature sensitive strain}

To study possible substrates of the TIM22 complex, Tim22 temperature sensitive (ts) yeast strains were utilised. Two ts strains were tested, tim22-F1 and tim22-14, carrying point mutations in the Tim22 locus. tim22-14 has already been characterised to an extent (Wagner et al., 2008). Growth of each strain was tested on fermentable glucose media and non-fermentable glycerol media, at various temperatures. At $25^{\circ} \mathrm{C}$, both strains grew similar to WT in glucose and glycerol media (Figure $27 \mathrm{~A}$ ). At $30^{\circ} \mathrm{C}$, tim22-F1 showed a moderate growth defect in glycerol, while tim22-14 grew similar to WT. At the nonpermissive temperature of $37^{\circ} \mathrm{C}$, the tim22-F1 strain showed a severe growth defect in both glucose and glycerol, while tim22-14 was less affected. To analyse the steady-state levels of proteins, mitochondria were isolated from all three strains after growing them at $25^{\circ} \mathrm{C}$, or at $25^{\circ} \mathrm{C}$ followed by $37^{\circ} \mathrm{C}$ for $14 \mathrm{~h}$. WT and tim22-14 showed similar levels for most proteins at the permissive $\left(25^{\circ} \mathrm{C}\right)$ temperature (Figure $27 \mathrm{~B}$, lane $\mathbf{1 , 2}$ vs lane 5,6 ), except for the mutant Tim22 protein, whose levels were down. At the non-permissive temperature $\left(37^{\circ} \mathrm{C}\right)$, tim22-14 showed reduced levels of $\mathrm{PiC}$ and $\mathrm{AAC}$ compared to the WT. Furthermore, Tim22 was not detected (Figure 27B, lane 3,4 vs lane 7,8). In contrast, the tim22-F1 mitochondria had no detectable levels of Tim22, $\mathrm{PiC}$ or AAC even at $25^{\circ} \mathrm{C}$. Moreover, levels of all other proteins were reduced compared to WT (Figure 27B, lane 1,2 vs lane 9,10$)$. Therefore, due to its growth defect, in combination with the reduced protein levels, this strain was not used for further studies. 


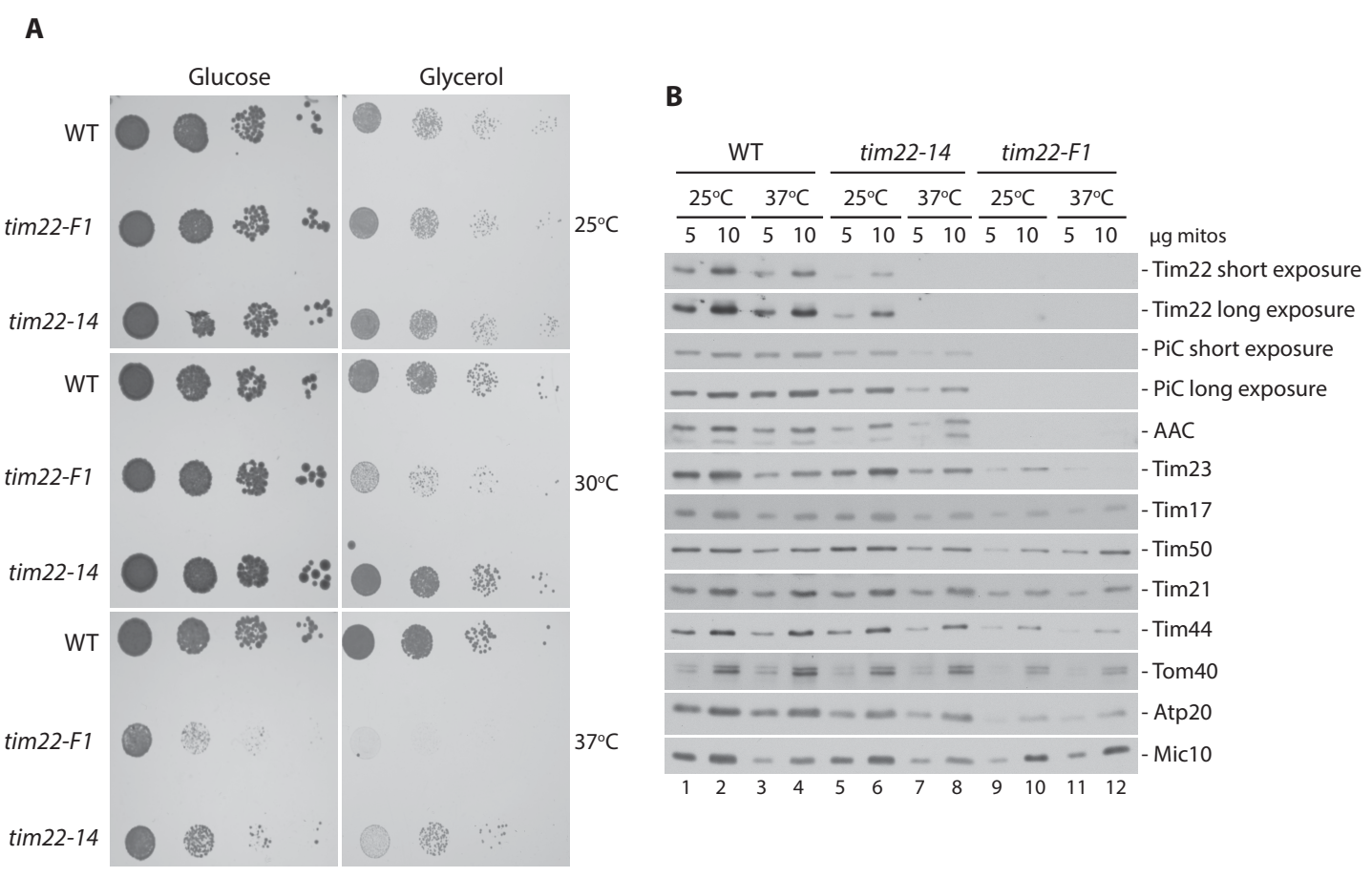

Figure 27 tim22-14 shows proper growth and Tim22 levels at permissive temperature A. WT and Tim22 temperature sensitive strains tim22-F1 and tim22-14 were grown on fermentable (left) and non-fermentable (right) media at 25,30 and $37^{\circ} \mathrm{C}$. B. Mitochondrial steady state levels of proteins in WT, tim22-F1 and tim22-14 yeast grown at permissive temperature $\left(25^{\circ} \mathrm{C}\right)$, or shifted to repressive temperature $\left(37^{\circ} \mathrm{C}\right)$ for $14 \mathrm{~h}$ were analysed by SDS-PAGE and western blotting.

\subsubsection{Temperature induced mislocalisation of known TIM22 complex substrates in the tim22-14 strain}

GFP-tagged TIM22 complex substrates would be expected to mislocalise from mitochondria upon growth in non-permissive conditions. To that end, $\mathrm{N}$ - and C-terminal GFP fusion constructs on yeast expression plasmid were generated for Mir1 (PiC) and AAC2 respectively. After transformation into WT and tim22-14, the strains were grown at permissive temperature, followed by a shift to non-permissive temperature for $25 \mathrm{~h}$. Protein localisation was analysed with fluorescence microscopy. For both WT and tim2214 strains, AAC2-GFP and GFP-Mir1 could be detected in mitochondria at $0 \mathrm{~h}$ at $37^{\circ} \mathrm{C}$, as seen by their co-localisation with MitoTracker (Figure 28, upper panel for both proteins). Upon a shift to the non-permissive temperature for $25 \mathrm{~h}$, proteins in both strains were affected differently. In WT cells, the proteins remained localised to mitochondria (Figure 28, lower panels on the left for both proteins). In the tim22-14 strain, however, a mislocalisation of the GFP signal from mitochondria was observed. AAC2-GFP could be detected at very low levels in mitochondria while the remaining protein was degraded. GFP-Mir1 was visible as punctate structures in the cell (Figure 28, lower panels on the right for both proteins). These could be aggregates of the non-imported protein. 
Therefore, abolishing the import pathway of carrier proteins led to their mislocalisation in tim22-14 cells.

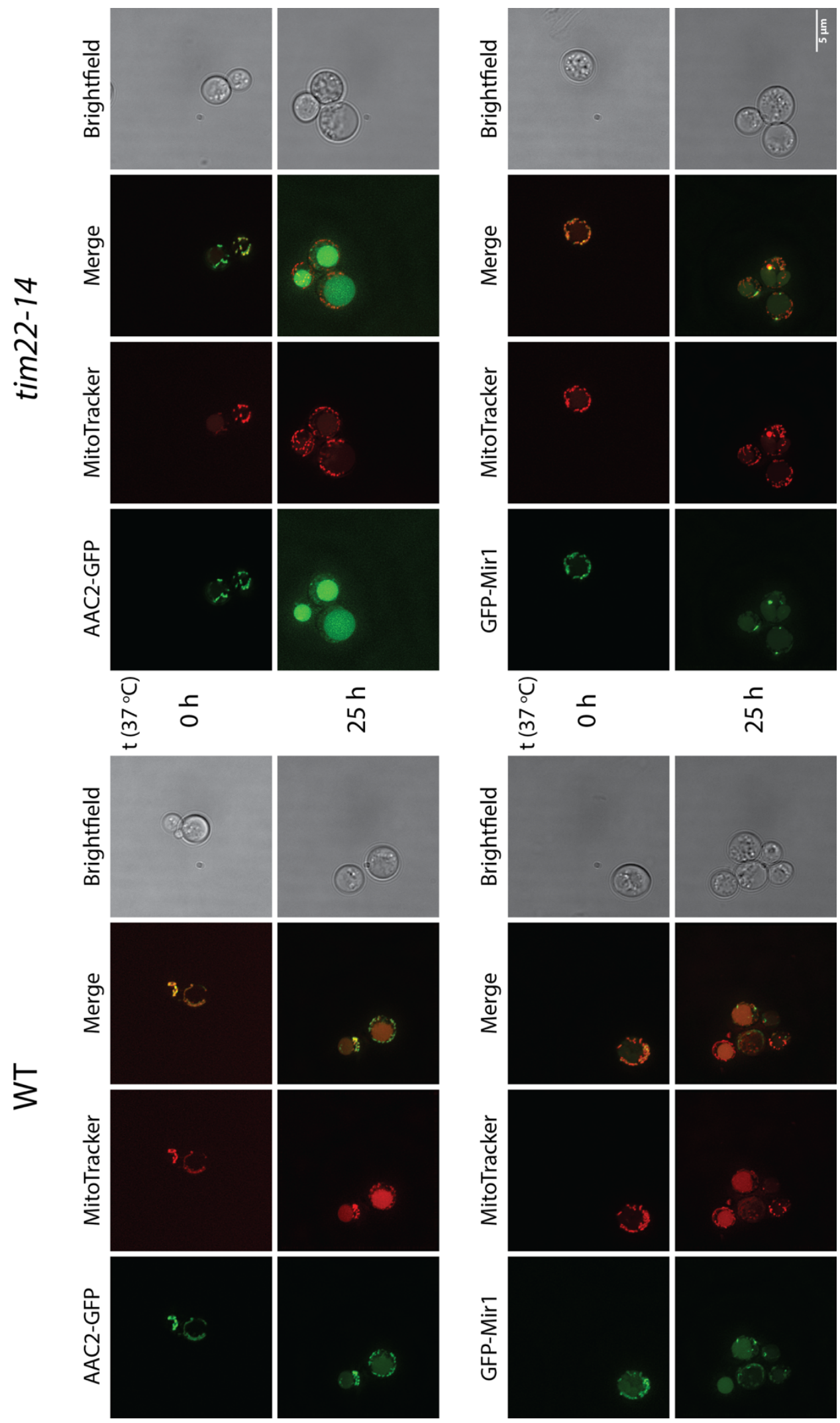

Figure 28 Carrier proteins (AAC2 and Mir1) mislocalise from mitochondria in the tim2214 strain under repressive growth conditions - Yeast WT and tim22-14 cells were 
transformed with plasmids encoding AAC2-GFP or GFP-Mir1. Cells were grown in SD medium at $30^{\circ} \mathrm{C}$ till late log phase and then shifted to $37^{\circ} \mathrm{C}$ for $25 \mathrm{~h}$. Samples were also co-stained with MitoTracker Red. Fluorescence microscopy was used for analysis (FITC and TRITC channels). Merged green and red fluorescence images are shown (yellow/orange). (Scale bar: $5 \mu \mathrm{m}$ )

\subsubsection{Mitochondrial inner membrane carrier and transporter proteins are specifically affected in tim22-14 mitochondria under non-permissive conditions}

\subsubsection{Mass spectrometric analysis to identify carrier substrates}

WT and tim22-14 cells were grown at $25^{\circ} \mathrm{C}$ to late log phase, followed by a shift to the non-permissive temperature for 15, 25 and $40 \mathrm{~h}$. Mitochondria were isolated from all eight conditions. This was followed by differential stable isotope dimethyl-based labelling (Hsu et al., 2003). Four mixtures were prepared. Each mixture contained differentially labelled mitochondria from both WT (light-L) and tim22-14 (medium-M or heavy-H) at a particular time point (Figure 29). Mix A had WT-L $0 \mathrm{~h}$, tim22-14-M $0 \mathrm{~h}$ and tim22-14-H $15 \mathrm{~h}$. Mix B had WT-L 15 h, tim22-14-M 0 h and tim22-14-H 15 h. Mix C had WT-L 25 h, tim22-14-M Oh and tim22-14-H 25 h. Mix D had WT-L 40 h, tim22-14-M Oh and tim22-14-H 40 h. Labelling and mass spectrometry analysis was performed by Dr. Ida Suppanz from the group of Prof. Dr. Bettina Warscheid (Biochemistry and Functional Proteomics, University of Freiburg). Heavy $(H) /$ light $(L)$ ratio was used to detect the specific effect of Tim22 mutation on mitochondrial proteins, while a heavy $(H) /$ medium $(M)$ ratio was a measure of the effect of heat stress within the tim22-14 strain. Overall, the dataset covered 637 out of $901(70 \%)$ proteins of the High Confidence Mitochondrial proteome (Morgenstern et al., 2017).

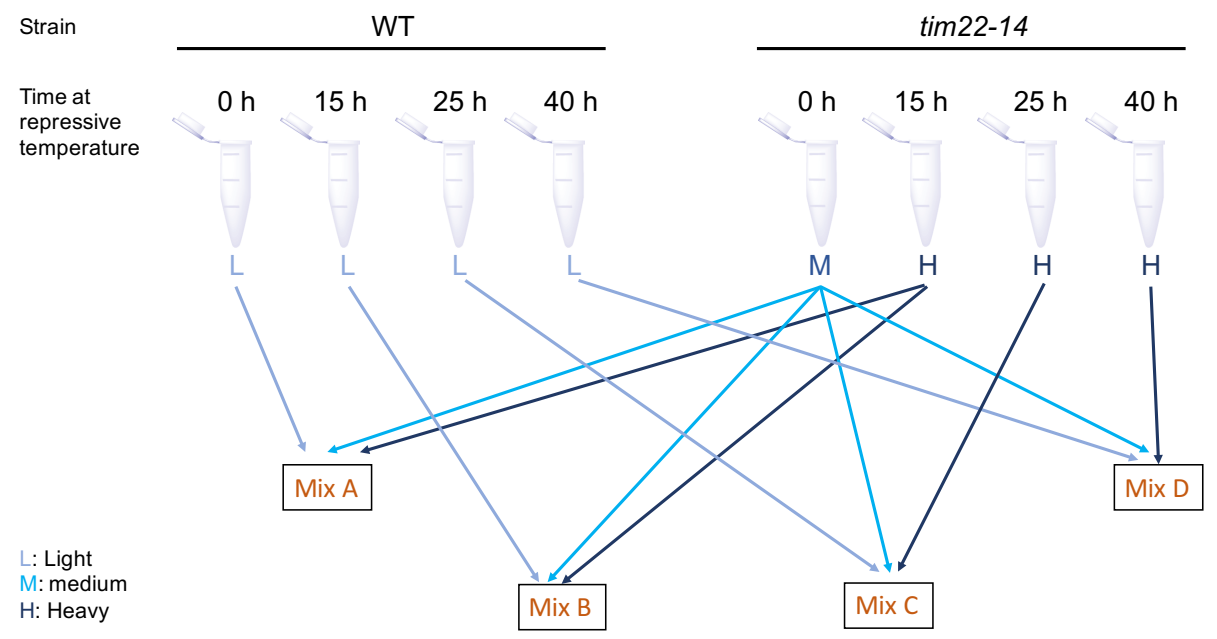

Figure 29 Mixing scheme for WT and tim22-14 mitochondria under different conditions Mitochondria were prepared from WT and tim22-14 yeast cells grown for $0,15,25$ and $40 \mathrm{~h}$ at $37^{\circ} \mathrm{C}$ after growing them to late log phase at $25^{\circ} \mathrm{C}$. Samples were mixed as indicated and subjected to triple dimethyl labelling, followed by mass spectrometry. 
Considering the $\mathrm{H} / \mathrm{L}$ labelling ratio, at time point $0 \mathrm{~h}$, no component of the TIM22 complex was visibly down-regulated. TIM23 and TOM complex subunits remained unaffected at all time points. At $15 \mathrm{~h}$ heat stress, Tim22 was more than 10x down-regulated. Additionally, among the most severely affected proteins were the mitochondrial inner membrane carrier and transporter proteins, whose levels were consistently reduced at all three time points. When all three heat stress time points (mix $B, C$ and $D$ ) were treated as replicates, a t-test revealed that most of these carrier proteins were significantly down-regulated in the mutant compared to WT (Figure 30). These putative substrate proteins are listed in Table 14.

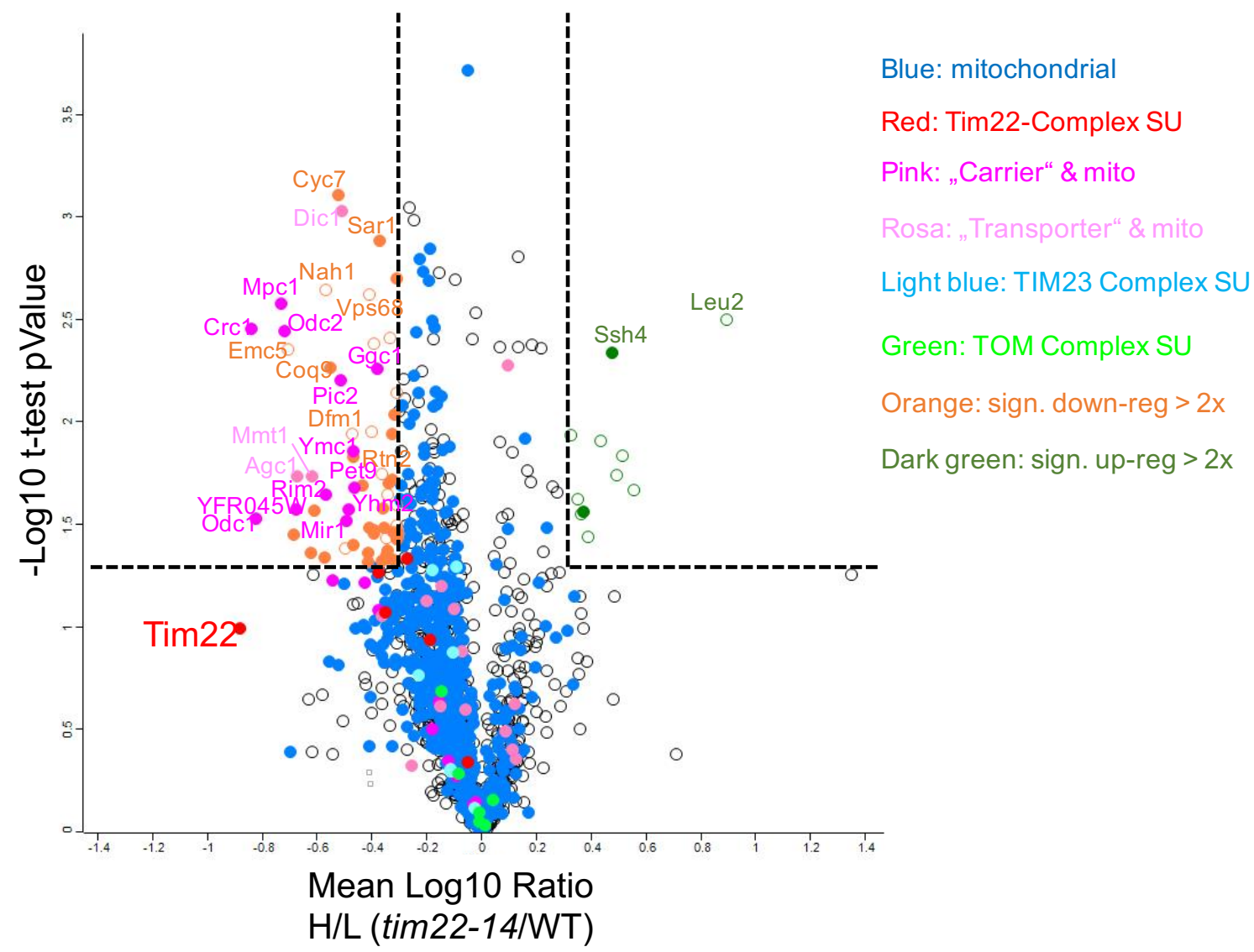

Figure 30 t-test overview of protein levels in tim22-14 and WT mitochondria after heat stress - Results from the three heat stress time points $(15,25$ and $40 \mathrm{~h}$ ) were treated as replicates to generate a combined t-test for mitochondrial proteins based on the $H / L$ ratio. Colour code indicates the protein sub-group. SU: subunit, mito: mitochondria, down-reg: down-regulated, upreg: up-regulated. 
Table 14 Carrier and transporter proteins most affected in tim22-14. MS analysis of differentially labelled WT and tim22-14 mitochondria, with average (BCD mixes) normalised $\mathrm{H} / \mathrm{L}$ ratio of $<0.5$.

\begin{tabular}{|l|l|l||}
\hline $\begin{array}{l}\text { Gene } \\
\text { name }\end{array}$ & Protein name & $\begin{array}{l}\text { Predicted TM } \\
\text { domains }\end{array}$ \\
\hline TIM22 & $\begin{array}{l}\text { Mitochondrial import inner membrane translocase } \\
\text { subunit TIM22 }\end{array}$ & 4 \\
\hline Carrier proteins & \multicolumn{2}{|l|}{} \\
\hline CRC1 & Mitochondrial carnitine carrier & 6 \\
\hline ODC1 & Mitochondrial 2-oxodicarboxylate carrier 1 & 6 \\
\hline MPC1 & Mitochondrial pyruvate carrier 1 & 2 \\
\hline ODC2 & Mitochondrial 2-oxodicarboxylate carrier 2 & 6 \\
\hline YFR045W & Uncharacterised mitochondrial carrier YFR045W & 6 \\
\hline RIM2 & Mitochondrial carrier protein RIM2 & 6 \\
\hline & Uncharacterised mitochondrial carrier YPR011C & 6 \\
\hline PIC2 & Mitochondrial phosphate carrier protein 2 & 6 \\
\hline MIR1 & Mitochondrial phosphate carrier protein & 6 \\
\hline YHM2 & Citrate/oxoglutarate carrier protein & 6 \\
\hline YMC1 & Carrier protein YMC1, mitochondrial & 6 \\
\hline PET9 & ADP/ATP carrier protein 2 & 6 \\
\hline AAC1 & ADP/ATP carrier protein 1 & 6 \\
\hline GGC1 & Mitochondrial GTP/GDP carrier protein 1 & 6 \\
\hline MPC3 & Mitochondrial pyruvate carrier 3 & 3 \\
\hline Transporter & 6 \\
\hline MMT1 & Mitochondrial metal transporter 1 & 6 \\
\hline AGC1 & Mitochondrial aspartate-glutamate transporter AGC1 & 6 \\
\hline DIC1 & Mitochondrial dicarboxylate transporter & 6 \\
\hline YDL119C & $\begin{array}{l}\text { Solute carrier family 25 member 38 homolog- HEM25 } \\
\text { glycine transporter }\end{array}$ & 6 \\
\hline OAC1 & Mitochondrial oxaloacetate transport protein & 6 \\
\hline SFC1 & Succinate/fumarate mitochondrial transporter & 6 \\
\hline & & 6 \\
\hline
\end{tabular}

This analysis was not without its secondary effects. Among the down-regulated proteins were also respiratory chain complex III and IV components. Subunits of these complexes require co-factors for their stability and assembly and could therefore be affected by the reduced levels of metabolite carriers in the tim22-14 mitochondria (Dufay et al., 2017). Considering the $\mathrm{H} / \mathrm{M}$ ratio, which represents the comparison of tim22-14 at $37^{\circ} \mathrm{C}$ for different time points vs $0 \mathrm{~h}$, it was observed that not only mitochondrial carriers and transporters, but also other proteins were affected. These also included components of 
TOM and TIM23 complexes. This suggested that the temperature shift to $37^{\circ} \mathrm{C}$ led to more pleiotropic effects in both WT and tim22-14.

\subsubsection{Steady-state analysis of mitochondrial proteins after heat shock}

Mass spectrometric analyses revealed the down-regulation of proteins of the carrier and transporter family. Steady-state protein levels were analysed for mitochondria prepared from WT and tim22-14 cells grown at $0,15,25$ and $40 \mathrm{~h}$ at $37^{\circ} \mathrm{C}$ (Figure 31).

Tim22 levels were moderately reduced at the $0 \mathrm{~h}$ time point in the tim22-14 samples compared to WT. However, at higher time points at non-permissive temperature, Tim22 could not be detected in the tim22-14 strain, in accordance with the mass spectrometric results which showed $>10 x$ down-regulation. The phosphate carrier $(\mathrm{PiC})$, citrate and oxoglutarate carrier (Yhm2) and the ADP/ATP carrier (AAC) all had reduced levels of protein in the tim22-14 strain with increasing time at $37^{\circ} \mathrm{C}$. Pyruvate carrier subunit, Mpc1, was reduced already without any heat shock in the Tim22 mutant strain. Other known substrates of the TIM22 complex, Tim23 and Tim17, did not show a significant reduction at any time point compared to WT. This could be expected because these are highly essential core components of the TIM23 complex, and their turnover would be quite low. Other components of the TIM22 complex (Tim54, Tim18), TOM complex (Tom40), TIM23 and PAM complexes (Tim50, Tim44, Hsp70), Complex V (Atp20) and MICOS (Mic10) were not affected, highlighting the specificity of this approach.

For most of the proteins, a temperature specific decrease was observed in both strains, suggesting a more secondary effect due to heat stress, which was taken into account while preparing the samples for labelling. Thus, a range of inner membrane proteins were detected to be specifically depleted in a Tim22 mutant strain. 


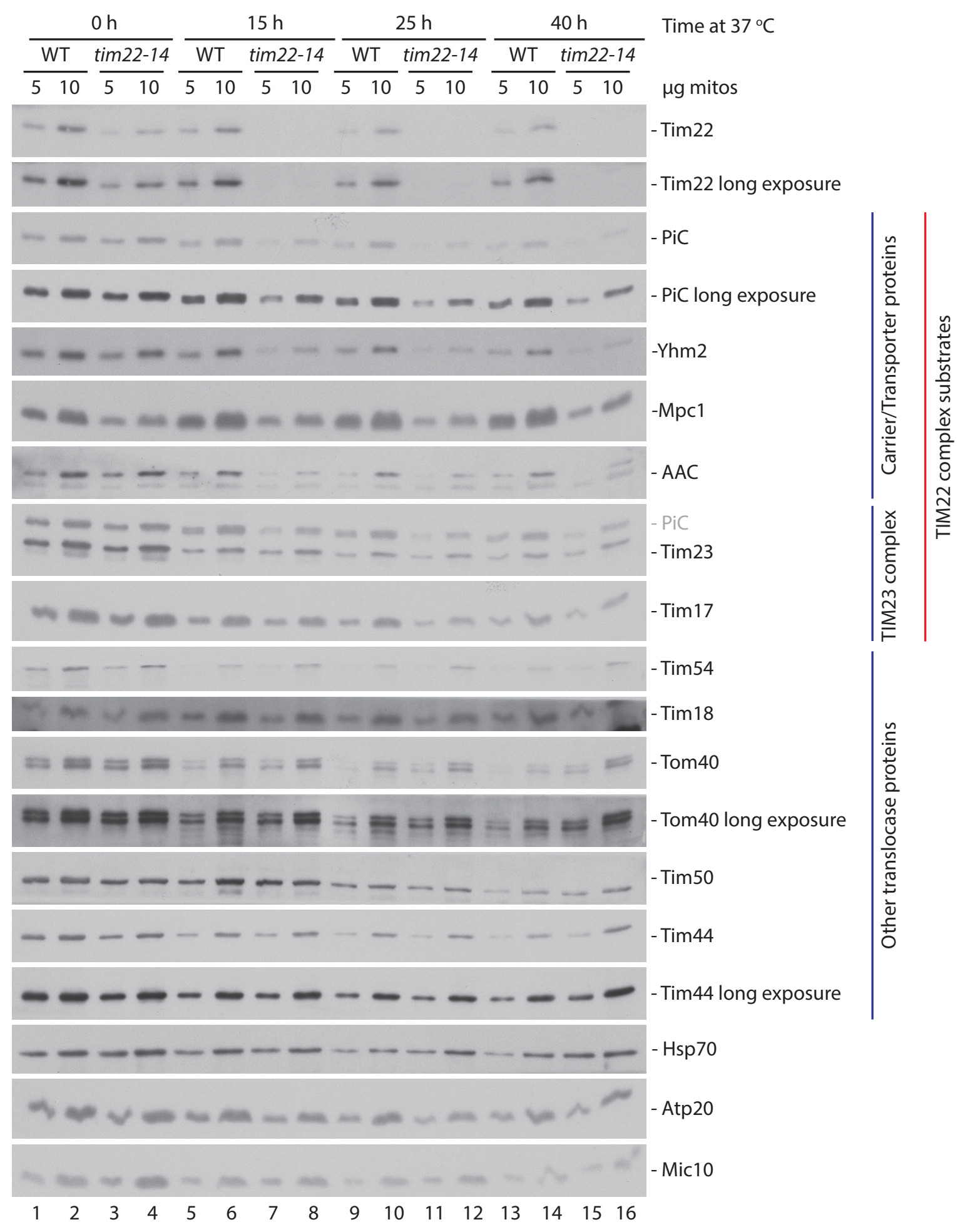

Figure 31 TIM22 complex substrate levels specifically decrease in tim22-14 mitochondria upon heat stress - Mitochondrial steady state levels of proteins in WT and tim2214 yeast grown at permissive temperature $\left(25^{\circ} \mathrm{C}\right)$ or shifted to repressive temperature $\left(37^{\circ} \mathrm{C}\right)$ for 15,25 and $40 \mathrm{~h}$ were analysed by SDS-PAGE and western blotting. 


\subsubsection{Import analysis of identified substrates}

\subsubsection{TIM23 complex substrate import is not affected in tim22-14 mitochondria}

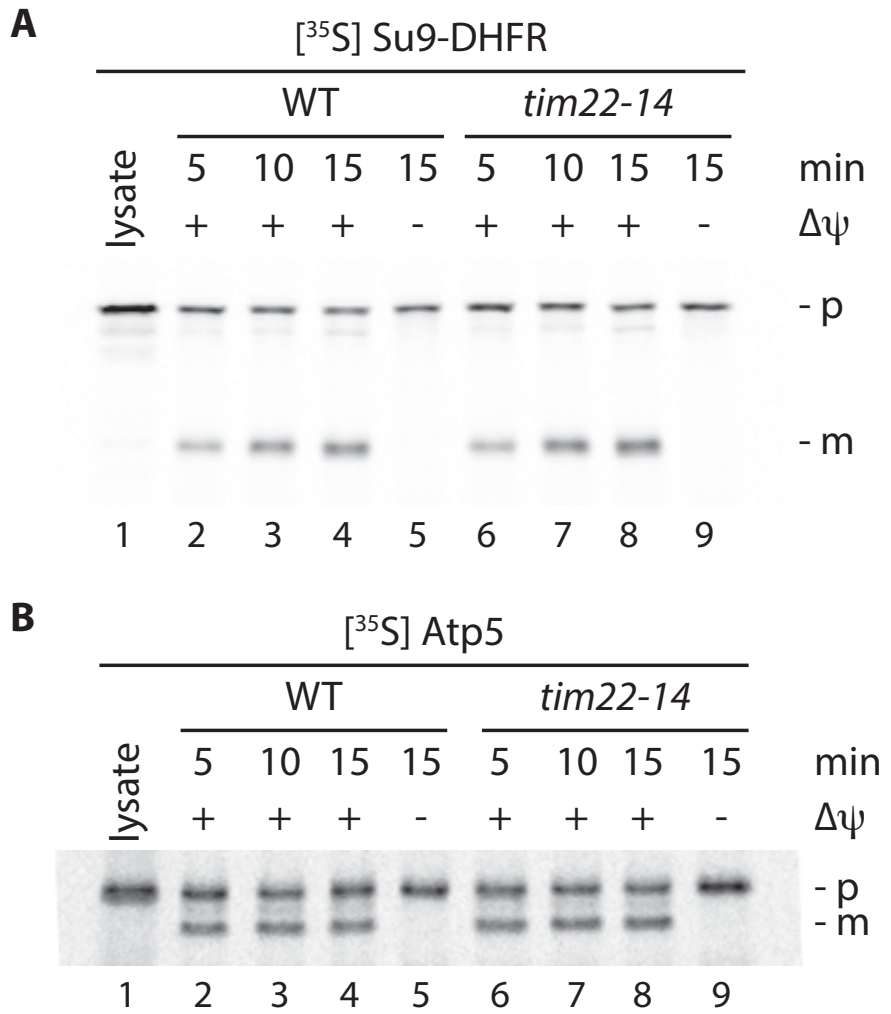

Figure 32 Import of TIM23 complex substrates is not affected in tim22-14 mitochondria$\left[{ }^{35}\right.$ S] labelled A. F1 $\beta$ and B. Atp5 was imported for the indicated time points into WT and tim22-14 mitochondria. The reaction was stopped by addition of AVO. Samples were analysed by SDSPAGE and autoradiography. $p$ - precursor, $m$ - mature form. AVO - mixture of $10 \mathrm{mM}$ Antimycin A, 1 $\mathrm{mM}$ Valinomycin and $10 \mathrm{mM}$ Oligomycin.

For several putative carrier substrates selected from the screen, it was decided to test their import and assembly into mitochondria to verify their dependence on TIM22. To rule out any indirect effects of the Tim22 mutant on substrates of the TIM23 complex, which utilise a presequence, import of Su9-DHFR and Atp5 was carried out. ${ }^{35} \mathrm{~S}$ labelled Nterminal part of the complex $V$ subunit 9 fused to DHFR was synthesised (Figure 32A, lane 1). When this precursor was incubated with mitochondria isolated from WT and tim22-14 for increasing time points, a faster migrating processed mature $(\mathrm{m})$ form was generated, demonstrating efficient import and processing. Time-dependent kinetic increase could be observed, and both mitochondria were equally competent for import of a presequence-containing protein (Figure 32A, lane 2,3,4 vs lane 6,7,8). No mature form could be detected in the absence of a membrane potential (Figure 32A, lane 5 and lane 
9). Similarly, for ${ }^{35} \mathrm{~S}$ labelled Atp5 of complex V, another TIM23 complex substrate, import efficiency into WT and tim22-14 mitochondria was similar (Figure 32B).

This Tim22 mutant strain has previously been reported to have only slightly reduced membrane potential compared to WT (Wagner et al., 2008). Therefore, any import defect due to a loss of membrane potential can be excluded.

\subsubsection{Assembly of carrier proteins is affected in tim22-14 mitochondria}

To monitor the import and assembly of selected carrier proteins from the MS analysis, blue native electrophoresis was used to differentiate between the stage III assembled and fully assembled protein. Proteins were divided into three sub-groups: (i) Carrier proteins with standard six transmembrane topology (Crc1, Odc1, Mir1, Hem25) (ii) Uncharacterised proteins potentially belonging to the carrier family of proteins (YFR045W, YPR011C) and (iii) Proteins with unusual number of transmembrane domains for carrier substrates (MPC1, MPC3). MPC1 and MPC3 have 2 and 3 predicted transmembrane domains each.

For proteins from (i) and (ii), ${ }^{35} \mathrm{~S}$ labelled precursors were imported and assembled into heat-shocked WT and tim22-14 mitochondria. Crc1 (Carnitine carrier) assembly was strongly reduced in tim22-14 mitochondria compared to WT mitochondria (Figure 33A, lane $1,2,3$ vs lane $5,6,7$ ). The complex did not assemble in the $\Delta \Psi$ control (Figure 33A, lane 4 and 8). For Odc1 (OxoDicarboxylate carrier), the decrease in import and assembly was not as strong, but still evident (Figure 33B, lane 1,2,3 vs lane 5,6,7). Assembly of glycine transporter Hem25 (HEMe synthesis by SLC25 family member) was strongly inhibited in the Tim22 mutant (Figure 33C, lane 1,2,3 vs lane 5,6,7). Mir1 or Phosphate carrier $(\mathrm{PiC})$ subunit assembly was also reduced, but not to a great extent (Figure 33D,

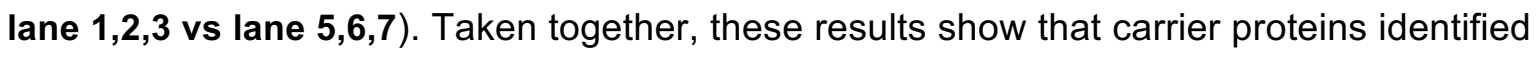
from the quantitative MS analysis are indeed substrates of the TIM22 complex. These proteins are affected to varying degrees in their assembly, which could be due to differences in the affinity of their internal signals for the TIM22 complex.

One of the uncharacterised proteins identified in the screen, YFR045W, also has six predicted transmembrane segments and is a putative mitochondrial transporter protein (Belenkiy et al., 2000). Import and assembly of ${ }^{35} \mathrm{~S}$ YFRO45W was carried out as described before. Strong assembly defects were observed (Figure 34, lane 1,2,3 vs lane $\mathbf{5 , 6 , 7 )}$, leading to the conclusion that YFRO45W is also a TIM22 complex substrate. 
A

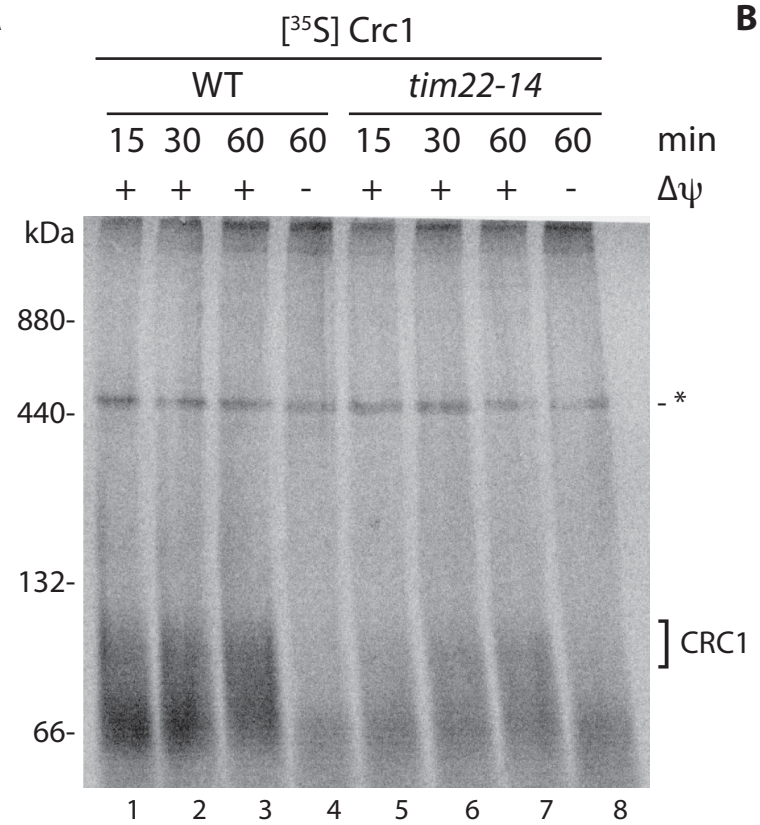

C

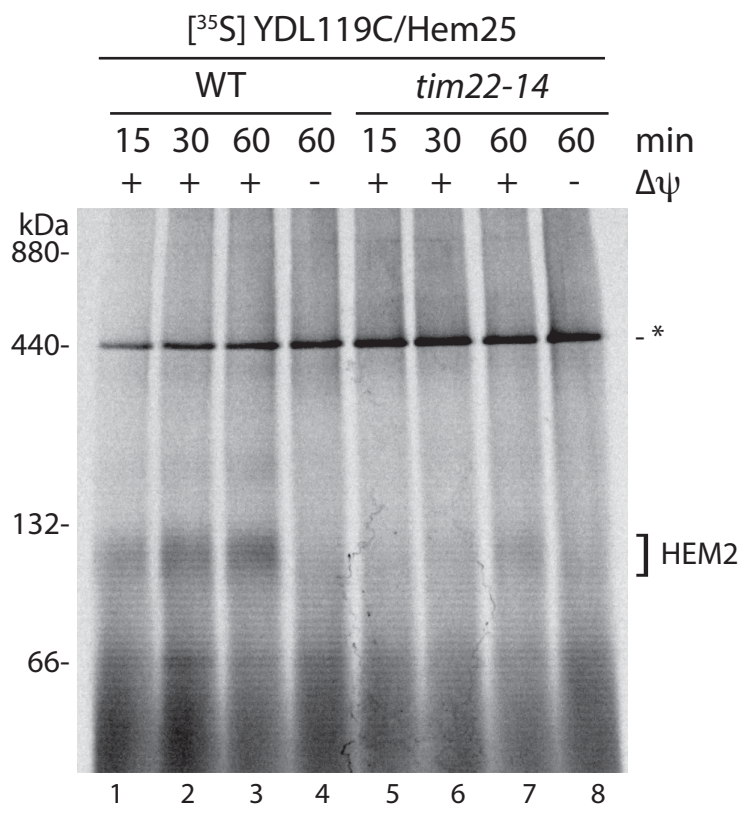
$\left[{ }^{35} \mathrm{~S}\right]$ Odc1

$\frac{\text { WT }}{15306060} \frac{\operatorname{tim} 22-14}{153060 \quad 60} \min$

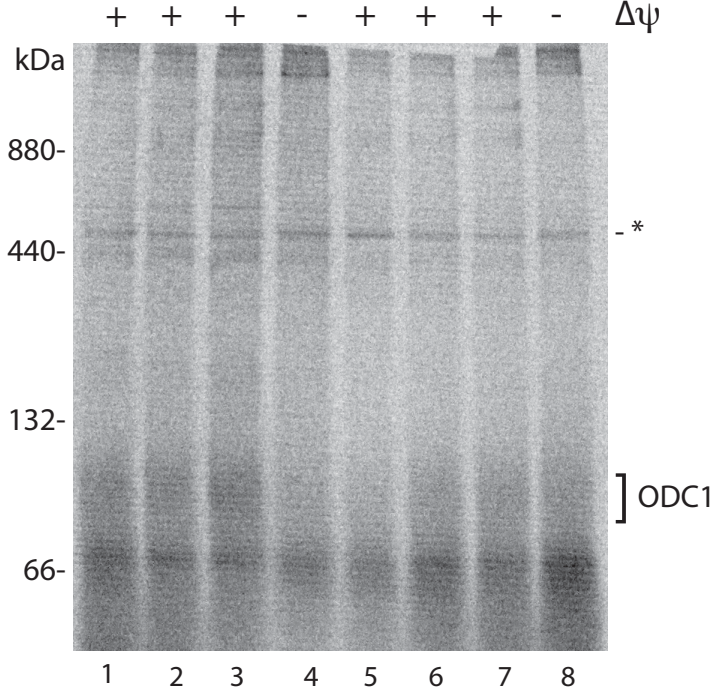

D $\left.{ }^{35} \mathrm{~S}\right]$ Mir1

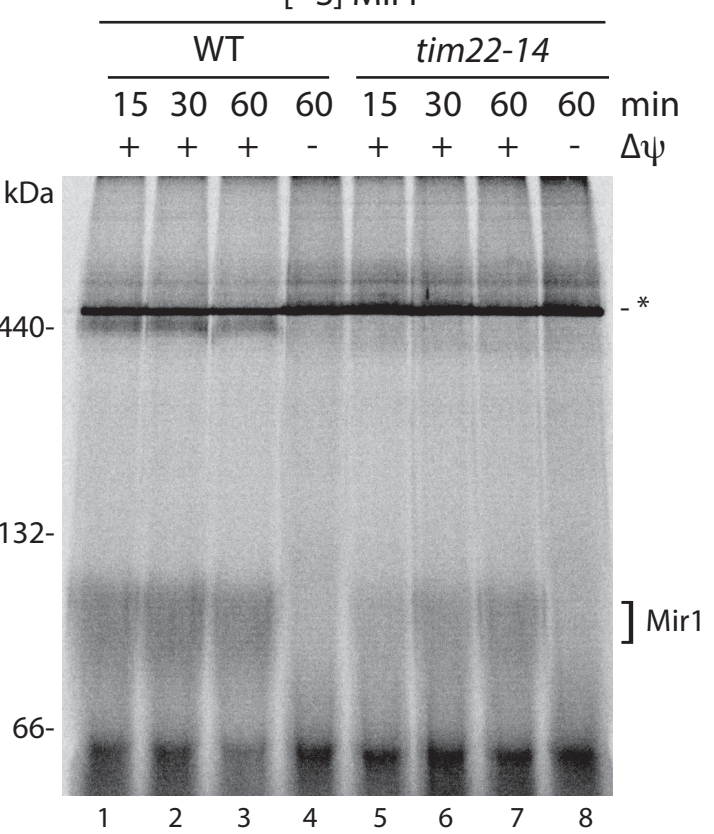

Figure 33 Assembly of carrier proteins is affected in tim22-14 mitochondria $-\left[{ }^{35} \mathrm{~S}\right]$ labelled A. Crc1, B. Odc1, C. Hem25 and 4. Mir1 was imported for 15, 30 and 60 min into WT and tim22-14 mitochondria to assess the assembly of carrier proteins. The reaction was stopped by addition of AVO followed by PK digestion. Assembly of the carrier was monitored on BN-PAGE followed by autoradiography. -*: non-specific signal. AVO - mixture of $10 \mathrm{mM}$ Antimycin A, $1 \mathrm{mM}$ Valinomycin and $10 \mathrm{mM}$ Oligomycin.

YPR011C is characterised to be a transporter of adenosine 5'-phosphosulfate in yeast (Todisco et al., 2014). ${ }^{35} \mathrm{~S}$ YPR011C was imported and assembled into WT and tim22-14. Assembly was reduced in the Tim22 mutant, indicating that YPR011C is also dependent on the TIM22 complex for its import (Figure 35 , lane $\mathbf{1 , 2 , 3}$ vs lane $5,6,7$ ). 


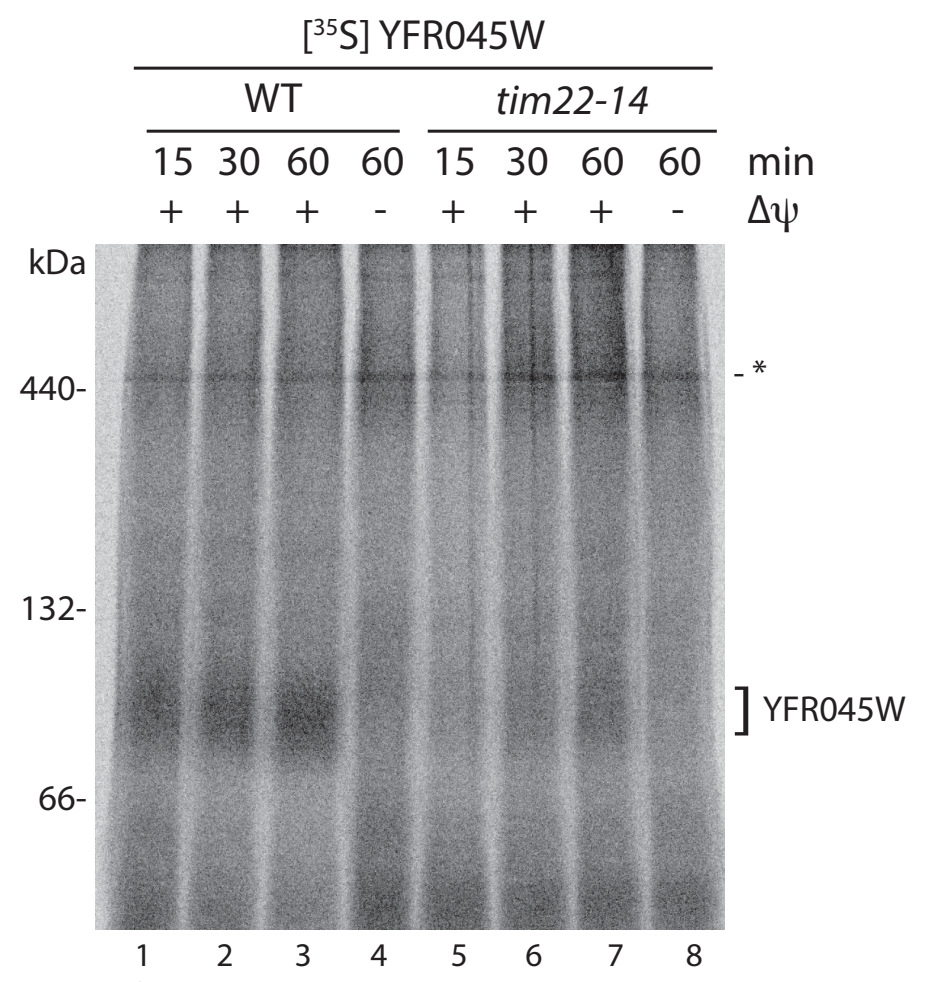

Figure 34 Assembly of uncharacterised carrier protein YFR045W is affected in tim22-14 mitochondria - $\left[{ }^{35} \mathrm{~S}\right]$ labelled YFR045W was imported for 15,30 and 60 min into WT and tim2214 mitochondria to assess its assembly. The reaction was stopped by addition of AVO followed by PK digestion. Assembly was monitored on BN-PAGE followed by autoradiography. -*: non-specific signal. AVO - mixture of $10 \mathrm{mM}$ Antimycin A, $1 \mathrm{mM}$ Valinomycin and $10 \mathrm{mM}$ Oligomycin.

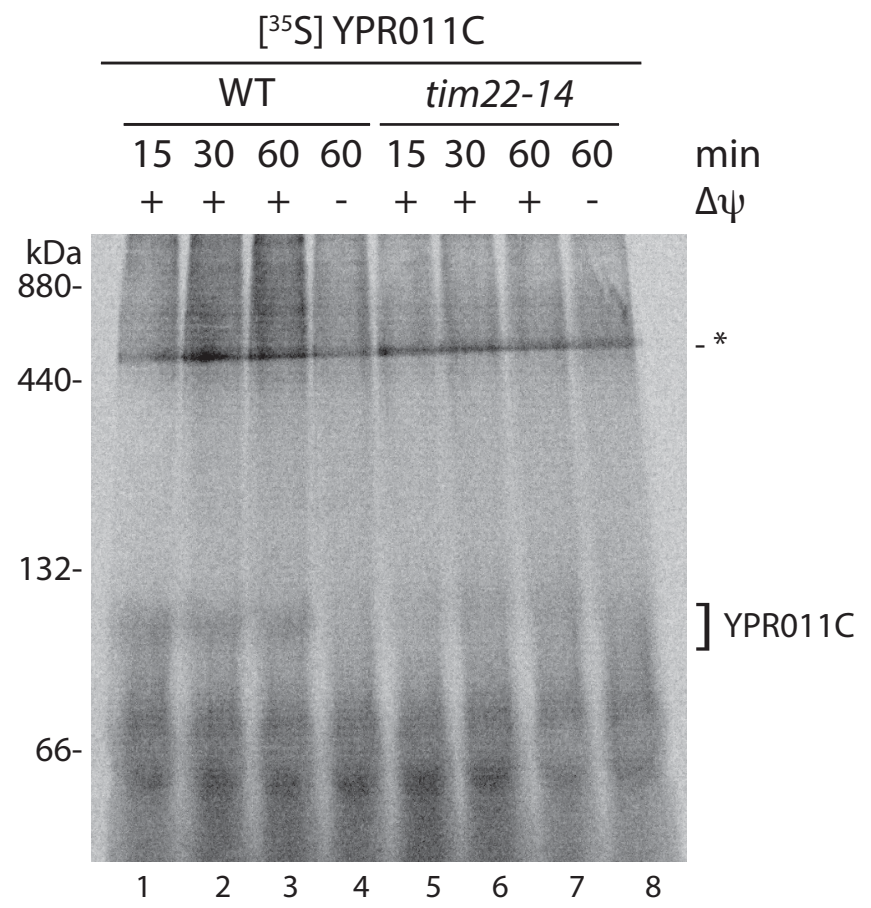

Figure 35 Assembly of uncharacterised protein YPR011C is reduced in tim22-14 mitochondria $-\left[{ }^{35} \mathrm{~S}\right]$ labelled YPR011C was imported for 15,30 and 60 min into WT and tim22-14 mitochondria to assess its assembly. The reaction was stopped by addition of AVO followed by PK digestion. Assembly was monitored on BN-PAGE followed by autoradiography. -*: non-specific signal. AVO - mixture of $10 \mathrm{mM}$ Antimycin A, $1 \mathrm{mM}$ Valinomycin and $10 \mathrm{mM}$ Oligomycin. 
Taken together, these results confirm some of the established carrier family proteins such as phosphate carrier ( $\mathrm{PiC})$, carnitine carrier ( $\mathrm{Crc1}$ ), glycine transporter (Hem25) and 2oxodicarboxylate carrier (Odc1) as substrates of the TIM22 complex. Additionally, uncharacterised proteins YFR045W and YPR011C, which have previously been reported to belong to the carrier family of proteins by proteomic studies, were confirmed as substrates of the TIM22 complex. Therefore, this quantitative mass spectrometry based approach helped in unravelling a set of inner membrane proteins which depend on TIM22 for their import. 


\section{Discussion}

\subsection{Analysis of a mitochondrial translocation intermediate: the TOM-TIM23 supercomplex}

For the mitochondrial presequence pathway, high-resolution structural information has been accumulated over the years for the soluble domains of various components (Albrecht et al., 2006) (Mokranjac et al., 2006) (Josyula et al., 2006) (Qian et al., 2011). Additionally, cross-linking analyses have shed light on the structural organisation of different subunits with respect to each other (Alder et al., 2008) (Shiota et al., 2011) (Lytovchenko et al., 2013) (Shiota et al., 2015). Recently, electron microscopy was utilised to resolve the structure of the TOM complex in N. crassa (Bausewein et al., 2017). However, to date, no high-resolution information is available on the TIM23 complex protein-conducting channel. Structural characterisation of this complex will provide a platform to integrate the functional information acquired so far with the structure of various subunits. This study was focussed on the isolation of the TOM-TIM23 supercomplex for structural analysis, as well as the examination of the dynamic interactions of TIM23 complex subunits in its unoccupied state versus a supercomplex state.

\subsubsection{Supercomplex forming proteins as a tool to study active protein import in mitochondria}

The small size, dynamic nature and intrinsic ability of the TIM23 complex to associate with other proteins makes it a challenging sample for structural studies using EM. One way to overcome this is by driving the TIM23 complex towards a stable TOM-TIM23 supercomplex form in the presence of an arrested translocating substrate. To date, $\mathrm{b}_{2}(167)_{\triangle}$-DHFR is the traditional protein which is used to generate the TOM-TIM23 supercomplex (Dekker et al., 1997) (Chacinska et al., 2003). In this study, new proteins were designed to optimise supercomplex generation and its purification on preparatory scale. All proteins had superfolder GFP at the C-terminus. The rationale behind the use of sfGFP was that a fast-folding blocking domain would be advantageous in case of an in 
vivo approach to generate the supercomplex. Based on our results, both SCGP and Jac1sfGFP could be purified in high amounts and formed the supercomplex more efficiently than $b_{2}(167)_{\Delta}$-DHFR. However, Jac1-sfGFP was selected as the protein for further experiments because of its higher stability during purification. Supercomplex isolation with anti-GFP nanobody resulted in co-isolation of TOM, TIM23 and PAM complex components. Therefore, new proteins for efficient formation of the TOM-TIM23 supercomplex were established in this study.

In parallel, an in vivo approach was also utilised. Here, Jac1-sfGFP was expressed in live yeast cells for a fixed time to accumulate the precursor in the translocase. Following this, cells were lysed open and the supercomplex was purified with the help of GFP nanobody. The advantage of this approach was to avoid time-consuming steps for preparing the mitochondria and carrying out an import reaction. However, this approach resulted in a lower supercomplex yield compared to the in organello isolation (data not shown). We hypothesise that since expression of Jac1-sfGFP might be toxic for cells, a mechanism might exist in the cell to clear the blocking protein from the import channels. A similar mechanism exists for clogged translocon in the ER, where Ste24, a metalloprotease, cleaves the clogging protein (Ast et al., 2016). Additionally, cytosolic quality control protein Vms1 was reported to antagonise aggregation of ribosome-stalled proteins in the mitochondrial translocases (Izawa et al., 2017). Recently, a surveillance mechanism, mitoCPR, was also reported for mitochondrial import defects (Weidberg and Amon, 2018), where stalled proteins in the translocase are removed by a AAA ATPase Msp1 on the OM. In light of these pathways in the cell, the in organello approach for supercomplex isolation was adopted in favour of the in vivo approach.

\subsubsection{Optimisation of the TOM-TIM23 supercomplex isolation conditions}

To date, the TOM-TIM23 supercomplex isolation has predominantly been carried out using tags on either a TOM or TIM23 complex subunit (Geissler et al., 2002) (Chacinska et al., 2003) (Chacinska et al., 2010). While efficient, this strategy could not be used directly for structural analysis due to the heterogeneous nature of the complex isolated, since both the TOM and the TIM23 complex associate with other complexes (Qiu et al., 2013) (Kulawiak et al., 2013) (Mehnert et al., 2014). With that in mind, GFP nanobody was purified to specifically isolate complexes in which Jac1-sfGFP was arrested. Free Jac1sfGFP was not co-isolated due to introduction of a mitochondria washing step after import of Jac1-sfGFP. The Jac1-sfGFP - GFP nanobody combination improved the supercomplex isolation, but initial analysis of the sample under electron microscopy 
pointed towards a heterogeneous population of particles of varying shape and size. These particles were most likely individual TOM and TIM23 complexes which had disintegrated from the supercomplex during the isolation procedure. A two-step isolation strategy was also optimised, wherein first all TIM23 complexes were isolated using a tag on Tim23 (His-SUMOstar-Tim23), followed by specific isolation of the supercomplex using GFP nanobody. While this led to a discernible improvement in sample homogeneity with EM analysis, the overall sample still remained heterogeneous.

To further stabilise the isolated TOM-TIM23 complex, an approach known as GraFix was utilised (Kastner et al., 2008). In this method, individual protein complexes are stabilised in the presence of a mild fixative agent (glutaraldehyde) while undergoing glycerol gradient ultracentrifugation. This method is especially beneficial for fragile complexes or complexes with low copy number. When the TIM23 complex isolated after Jac1-sfGFP import was applied on a glycerol gradient for GraFix, a separation could indeed be observed for the unoccupied TIM23 complexes and the TOM-TIM23 supercomplex form, due to their different size. Analyses of fractions containing the supercomplex with EM revealed that while there was still some heterogeneity remaining, the particle size on average was larger. Therefore, sample preparation was gradually improved with various optimisations.

Detergent micelles were another issue that interfered with the EM analysis. Since the TIM23 complex is fragile in nature and known to be stable only in digitonin, it was the detergent of choice for isolations. However, the large digitonin micelle size led to difficulties in differentiating between protein particles and micelles under EM. Indeed, even recrystallisation of digitonin (Herrmann et al., 2001), which purifies the commercially available digitonin to a great extent, did not completely eliminate the background detergent particles. Extensive detergents and surfactants were previously tested as substitutes of digitonin (Lytovchenko O., 2012), but the TIM23 complex dissociated in all of them. In this study, two new detergents, GDN and LMNG (Chae et al., 2010) (Chae et al., 2012), as well as SMA copolymer for detergent-free membrane protein extraction (Dörr et al., 2016) were tested. All three have been shown to stabilise various membrane proteins. The TIM23 complex completely disintegrated in LMNG, and solubilisation efficiency with SMA was low. Remarkably, with GDN, the TIM23 complex remained stable after isolation and was comparable to isolations with digitonin. Indeed, it was visible on EM analysis that the background contamination was reduced after exchange of detergent from digitonin to GDN.

Naturally, the next step was to combine detergent exchange with GraFix. In this method, called GraDeR, free detergent micelles can be removed from a detergent-extracted membrane protein under mild conditions using glycerol gradient centrifugation (Hauer et 
al., 2015). Analysis of the supercomplex-containing fraction with EM revealed much less micellar structures compared to digitonin, indicating the efficiency of the detergent exchange. Simultaneously, the particle size remained large on average, pointing towards the stability of the supercomplex with this approach.

Taken together, we developed a new approach for isolating the TOM-TIM23 supercomplex in preparative amounts for structural analysis with EM. Jac1-sfGFP proved to form the supercomplex efficiently, and utilisation of SUMOstar protease and GFP nanobody further enhanced the isolation efficiency and specificity. GraFix and GraDeR together stabilise the supercomplex and prevent it from degrading. After these extensive optimisations, the next step would be to increase the yield of isolation for improved negative stain and cryo EM, to get structural insights. Localisation of subunits within the acquired structure could be carried out based on the known structural information of some of the proteins (Endo et al., 2011) as well as by performing supercomplex isolations from strains with deletion or down-regulation of specific subunits. Another approach which can enhance the knowledge of spatial orientation of different subunits within a complex is cross-linking. This will be discussed in the next section.

\subsubsection{Cross-linking analyses of the mitochondrial TOM-TIM23 supercomplex}

Current view holds that after traversing the TOM complex, the presequence of a precursor proteins associates with Tom $22^{\mathrm{IMS}}$. The binding of $\mathrm{Tim} 21^{\mathrm{IMS}}$ to Tom $22^{\mathrm{IMS}}$ leads to dissociation of the precursor protein from Tom22 (Chacinska et al., 2005). Thereafter, the released presequence associates with Tim50, which is the major presequence receptor in the IM. Tim23 $3^{\mathrm{IMS}}$ can affect the Tim50 $0^{\mathrm{IMS}}-\mathrm{Tim}_{2} 1^{\mathrm{IMS}}$ interaction (Lytovchenko et al., 2013). The Tim50-Tim23 interaction causes the presequence to be released from Tim50, following which it can be handed over to the Tim23-Tim17 channel.

Cross-linking followed by mass spectrometry has developed into a significant tool to determine protein interactions in dynamic and unstable protein complexes. Additionally, cross-linking information provides the perfect complement to structure analysis. With that in mind, we looked into the interactions occurring in the TOM, TIM23 and PAM complexes in the absence and presence of a translocating protein.

\subsubsection{Tim21 interacts with Tom22 in the presence of a translocation arrested protein}

Tim21 is an IM protein with a large domain exposed in the IMS. Tim2 $21^{\text {IMS }}$ is known to interact with the IMS domains of Tom22, Tim50 and Tim23, most likely facilitating the transfer of precursors from the TOM to the TIM23 complex. However, mitochondrial 
translocases are not completely dependent on these interactions, since Tim21 is not an essential protein and is dispensable for yeast growth (Chacinska et al., 2005).

In our study, we discovered that in the absence of a translocating protein, Tim21 forms cross-links with Tim50 ${ }^{\mathrm{CORE}}$ and Mgr2 in the IMS. The Tim21 $1^{\mathrm{IMS}}$ and Tim50 ${ }^{\mathrm{CORE}}$ domain interaction has previously been reported for recombinantly purified proteins (Lytovchenko et al., 2013), and is now confirmed by our in organello cross-linking approach. Therefore, in the absence of a presequence signal, Tim $21^{\mathrm{IMS}}$ and Tim50 ${ }^{\mathrm{IMS}}$ interact with each other. In the presence of a translocating protein, only a single cross-link was obtained, between Tim $21^{\text {IMS }}$ and the last residue of Tim50. This residue is in the PBD, whose structural information is not available. This can be due to two reasons: one, that Tim2 $21^{\mathrm{IMS}}$ or Tim50 IMS undergo a conformational change in the presence of a translocating peptide, such that the Tim50 PBD gets in proximity to Tim21 ${ }^{\mathrm{MMS}}$, or two, since the score of this cross-link was low, its reliability can be questioned. Nevertheless, further proximity based in vitro experiments have to be performed to address if the Tim50 ${ }^{\mathrm{IMS}}$ conformational change is actually happening in the presence of a translocating peptide.

Furthermore, cross-links were obtained between the $\mathrm{N}$-terminus of Mgr2 and Tim2 $1^{\mathrm{IMS}}$ under both conditions. Mgr2 is an IM protein most likely forming a part of TIM23 ${ }^{\text {CORE }}$. It has previously been reported to be required for the recruitment of Tim21 to the TIM23 complex (Gebert et al., 2012). Therefore, here we could show that Tim21 is in proximity to Mgr2 within the TIM23 complex, promoting its recruitment. Interestingly, the number of obtained cross-links between them decreased in the presence of a translocating protein. This could be due to the increased interaction of Tim2 $21^{\text {IMS }}$ with Tom22 IMS, leading to reduced number of interaction sites for Mgr2.

Finally, Tim $21^{\mathrm{IMS}}$ forms cross-links with Tom $22^{\mathrm{IMS}}$ in the presence of an arrested protein. While the in organello formation of a supercomplex with an arrested protein is an artificially generated system, it reflects the corresponding supercomplex formed by close apposition of the TOM and TIM23 complexes during import of protein in vivo. Therefore, cross-links between subunits of the TOM and the TIM23 complex were expected. Interaction of Tom2 $2^{\mathrm{IMS}}$ with IMS domains of Tim21, Tim23 and Tim50 has been reported (Albrecht et al., 2006) (Tamura et al., 2009) (Bajaj et al., 2014a) (Waegemann et al., 2015). For us, cross-links were obtained only between Tim21 and Tom22. The absence of cross-links between Tim23-Tom22 or Tim50-Tom22 could be due to the efficiency of cross-linking, the nature of cross-linker or the loss of interactions during the isolation procedure.

Negatively charged amino acids on a 17-residue segment of Tom22 $2^{\text {IMS }}$ interact with Tim21 (Albrecht et al., 2006). However, what are the residues on Tim21 which act as the scaffold 
for Tom22? Our study identified Tim21 residues in proximity with Tom22 in the IMS. These residues are part of the $\beta$-sheet and inter-sheet loops (Figure 25) in Tim2 $21^{\mathrm{IMS}}$. Together, these represent an area of positively charged residues on the surface, which could potentially interact with Tom $22^{\mathrm{IMS}}$ via electrostatic interactions. Site-directed mutation analysis has to be performed to pin-point if the pocket formed by these residues is indeed the Tom22 binding site.

\subsubsection{Tim23 is in proximity to the TOM complex in the IMS}

Tim23 plays a crucial role in the translocation of a presequence-containing protein across the IM. It is a major subunit of the TIM23 complex, consisting of the N-terminal IMS domain (1-96) and the C-terminal membrane domain (97-222). The IMS domain is intrinsically disordered in nature (la Cruz et al., 2010) and acts as a hub for interacting with various proteins within the IMS. However, contradictory results exist regarding the functional relevance and topology of $\operatorname{Tim} 23^{\mathrm{IMS}}$. On the one hand, it has been reported that the N-terminus of Tim23 $3^{\mathrm{IMS}}$ (1-50) spans the outer membrane, with 20 amino acids exposed in the cytosol, such that the remaining IMS domain (51-96) is in proximity to the TOM complex to facilitate protein transfer (Donzeau et al., 2000). On the other hand, it has also been reported that these 50 residues at the extreme $\mathrm{N}$-terminus of $\operatorname{Tim} 23$ are dispensable for protein import and the TOM-TIM23 supercomplex formation (Chacinska et al., 2003) (Chacinska et al., 2005).

According to our cross-linking results, $\mathrm{Tim} 23^{\mathrm{IMS}}$ is in close proximity to multiple proteins. Specifically, residue 25 interacts with IMS-exposed residues of Tom40 and Tom5, while intra-protein cross-linking occurs between residues 25 or 32 and residue 66 . These results indicate that the disordered Tim23 ${ }^{\mathrm{IMS}}$ domain dynamically interacts with multiple proteins from both the TOM and the TIM23 complexes within the IMS. This finding is partially corroborated by the view that Tim2 $3^{\mathrm{IMS}}$ might dynamically contact both the IM and the OM through 2 segments in its $\mathrm{N}$-terminus (residues 1-7, 29-46), while the non-membranebound residues interact with other translocase components (Bajaj et al., 2014b). A point of interest in our cross-linking analysis is the interaction of Tim23 with Tom5 and Tom40. These interactions have not been reported before, and are observed in both the unoccupied TIM23 and the TOM-TIM23 supercomplex. This suggests that Tim23 ${ }^{\text {IMS }}$ is in constant proximity to the TOM complex, which can help in efficient transfer of presequence proteins to the TIM23 complex.

Remarkably, from our study, Tim23 also cross-links with Tim50 in the IMS and Tim17 in the matrix. This is not surprising since these three proteins together form the core TIM23 complex. What was surprising was that all Tim23 interactions with other TIM23 complex 
subunits, including with itself since it forms a dimer, were absent in the TOM-TIM23 supercomplex. While the absence of a cross-link is not an indication of a lack of proximity, similar behaviour of Tim23 in the presence of a translocation arrested precursor has previously been reported, also through cross-linking analysis (Alder et al., 2008). All in all, our findings reproducibly indicate the dynamic nature of the conformationally flexible IMS domain of Tim23 in the absence or presence of a translocation intermediate.

\subsubsection{The dynamic nature of the PAM complex}

For complete translocation of a protein into the matrix, a motor complex is required to first engage the incoming protein and then to drive precursor movement and unfolding. Tim44, Hsp70 (Ssc1), Pam16, Pam18 and Mge1 act together to ensure proper translocation of an incoming protein. Due to this, a difference is expected between the arrangements and interactions of PAM complex subunits in the TIM23 $3^{\text {MOTOR }}$ state compared to the supercomplex state.

Tim44 acts as a scaffold for other PAM complex subunits to anchor them at the TIM23 complex. Therefore, cross-links between Hsp70 and Tim44 are not surprising. However, so far, only the Tim44 NTD has been reported to interact with Hsp70 (Craig, 2018). Based on our analysis, residues predominantly in the Tim44 NTD-CTD interface interact with Hsp70 SBD independent of the presence of a translocating protein. This interface has been implicated with being involved in the interaction of Tim44 with the inner membrane (Weiss et al., 1999) (Marom et al., 2009). The seemingly different regions of Tim44 found to interact with Hsp70 could be due to differences in complex isolation and cross-linking strategies. Another reason could be that the residues in this interface are too flexible (Josyula et al., 2006) (Ting et al., 2017), resulting in their random interactions with Hsp70. In addition, a lack of structural information for Hsp70 and the Tim44 NTD-CTD interface prevents us from confirming the reliability of these cross-links. However, point mutation of Pro 442 to Ser in Hsp70 was reported to alter its interaction with Tim44 (Strub, 2002). This supports the cross-link we observed of adjacent Lys 444 and 445 residues in Hsp70 with Tim44. Therefore, mutation analysis will provide an answer regarding the importance of the Tim44 NTD-CTD interface for Hsp70 binding in vivo.

Furthermore, interactions between Pam16 and Pam18 are a point of interest. These proteins act together to either directly stimulate (via Pam18) or indirectly inhibit (via Pam16) the ATPase activity of Hsp70 (Li et al., 2004). Based on the known domain topology and limited structural information (Mokranjac et al., 2006), the interaction we observed was occurring between the $\mathrm{J}$ and J-like domains of Pam18 and Pam16 respectively. Furthermore, the number of cross-links between these proteins increase in 
the presence of a translocating protein. While this could be a technical issue during mass spectrometry measurements, it could also point towards rearrangements within Pam16, Pam18, or both, leading to exposure of more Lys residues on the surface, making it more accessible to the cross-linker. This rearrangement would not be surprising, since typically, Pam16 inhibits the ATPase stimulatory activity of Pam18 by blocking its HPD motif. However, for active import of proteins, Pam18 HPD motif should be available to stimulate ATPase activity of Hsp70. Additionally, cross-links were also observed between Pam16Tim44 NTD and Pam18-Tim44 NTD. The Pam16-Pam18 heterodimer can be expected to be present in the vicinity of Tim44 to promote active protein import. The $\mathrm{N}$-terminus of Pam16 is known to interact with Tim44 NTD between residues 51 to 82 (Schilke et al., 2012). However, for us, the cross-link was obtained with residue 110. Since the NTD of Tim44 is typically disordered in nature, a different cross-linking and TIM23 complex isolation approach could affect its interaction with Pam16 differently. Therefore, the Tim44 NTD behaves as a scaffold for multiple interactions with PAM complex subunits, promoting their assembly at the TIM23 complex. The large number of interactions of Tim44 NTD could be explained by its intrinsically disordered nature, which makes it more flexible. Moreover, Tim44 CTD has been demonstrated to interact with the matrix loops of Tim23 and Tim17, both in intact mitochondria and through recombinantly purified proteins, most likely to localise Tim44 in proximity to the TIM23 complex (Ting et al., 2014) (Ting et al., 2017) (Banerjee et al., 2015). However, neither of these interactions could be observed in our analysis. It could be that the Tim23-Tim44 interaction is labile, such that when cross-linking is carried out in intact mitochondria followed by isolation of the TIM23 complex, Tim23-Tim44 cross-links are detected (Ting et al., 2014) (Ting et al., 2017). However, when the TIM23 complex is first isolated and then cross-linked, as is carried out in our approach, the Tim23-Tim44 interactions are lost. The seemingly increased interactions between motor proteins as part of the TOM-TIM23 supercomplex is supported by the results that the supercomplex is a dynamic entity undergoing constant remodelling of its subunits (Schulz and Rehling, 2014).

In summary, in the present study, we utilised an in vitro cross-linking approach on an isolated TIM23 and TOM-TIM23 supercomplex, to analyse the cross-talk between the subunits of these complexes. We also assessed possible changes in the dynamics of TIM23 and PAM complex subunits under the condition of active protein import. By doing so, we found the following: firstly, Tim $21^{1 \mathrm{MS}}$ and Tom $22^{\mathrm{IMS}}$ are in proximity during import of a precursor protein; secondly, Tim $23^{\mathrm{IMS}}$ is in the vicinity of the TOM complex, specifically to Tom40 and Tom5, in both its unoccupied and supercomplex form. Finally, the PAM complex subunits are dynamic and potentially undergo restructuring in the presence of a 
translocating protein. Therefore, the cross-linking approach can serve as a useful tool for membrane proteins, to obtain initial impressions about the protein interaction network. The identified cross-links will provide valuable information for future structural studies.

Apart from electron microscopy and cross-linking analysis, gross structural information of protein complexes in their native environment can also be obtained through electron tomography. Specifically, the TOM-TIM23 supercomplexes were reported to localise as clusters on the mitochondrial surface, most likely for a focussed protein import (Gold et al., 2014). Refinement of this approach to include larger datasets can provide additional information on the surface features of these complexes. Additionally, this approach can be adapted to study the TOM-TIM23 supercomplex behaviour under different metabolic and physiological conditions.

Together, these approaches can help to comprehensively understand the structure of the TIM23 complex or the TOM-TIM23 supercomplex. That, in turn, will open the mitochondrial import field regarding the stoichiometry and underlying mechanisms of different subunits involved in the import of presequence-containing proteins into mitochondria.

\subsection{Expansion of substrate spectrum of the TIM22 complex}

To date, the import of only a handful of nuclear-encoded mitochondrial proteins has been reported to occur via the TIM22 complex. These proteins belong either to the six transmembrane carrier family proteins (AAC, $\mathrm{PiC}, \mathrm{DiC})$ or the four transmembrane translocase subunits (Tim23, Tim22 and Tim17). The primary channel-forming protein of this complex is Tim22. In this study, we utilised a Tim22 temperature sensitive (ts) strain in combination with a mass spectrometry-based depletion assay, to identify numerous predicted carrier family proteins as substrates of the TIM22 complex. This approach can be used as a broad screening tool, since the proteomic analysis is done on a whole mitochondria level to uncover specific proteins whose levels are up or down-regulated under specific conditions. Recently, a similar approach has been used to successfully identify IM proteins which depend on OXA for import (Stiller et al., 2016).

\subsubsection{Mitochondrial carrier proteins are depleted in tim22-14}

To study the dependence of mitochondrial proteins on Tim22 and therefore the TIM22 complex, mitochondria from cells grown at repressive temperature for three different time points were analysed. Following labelling and MS analysis, numerous proteins were found to be down-regulated for all time points. Primarily, the known carrier family proteins could be identified, including proteins like Crc1, Odc1, Rim2, Pic2, Mir1, Yhm2 and AAC2. 
Carrier family proteins have been classified based on their six transmembrane topology, with the carrier signature (CS) playing a major role in import (Nelson et al., 1998) (Belenkiy et al., 2000) (Kunji, 2004) (Ferramosca and Zara, 2013). With import and assembly of these proteins, we could confirm that, indeed, these proteins depend on Tim22 for their import. Moreover, these proteins were dependent to different extents on Tim22 for their assembly. This variation could be due to the helicity of transmembrane domains of that protein or the degree of adherence of the CS motif. Extensive sequence analysis would have to be done to address this variation.

Interestingly, along with the known carrier proteins, two other subsets of proteins were obtained. One was a group of uncharacterised six transmembrane predicted proteins, including YFR045W and YPR011C. The assembly of these proteins was diminished in the Tim22 mutant strain, indicating that they are substrates of the TIM22 complex. Remarkably, proteins with atypical transmembrane domains were also detected to be Tim22 dependent. These include proteins of the mitochondrial pyruvate carrier: Mpc1 and Mpc3. Another subunit, Mpc2, which is topologically and sequentially similar to Mpc3, was not obtained in the MS results. This absence could be due to the oxidative growth conditions of yeast strain, whereas $\mathrm{Mpc} 2$ is expressed under fermentative growth conditions (Bender et al., 2015). Mpc1 is a two-transmembrane protein, whereas Mpc2 and Mpc3 contain three transmembrane domains each.

With this method, few points had to be contemplated: First of all, only $70 \%$ of the mitochondrial proteome was covered with this approach, based on the High Confidence Mitochondrial proteome (Morgenstern et al., 2017). Therefore, the remaining 30\% proteins could also harbour additional known, uncharacterised or atypical TIM22 complex substrates. However, the feasibility of this approach is confirmed by obtaining known TIM22 complex substrates as down-regulated proteins in the Tim22 mutant strain. Secondly, such assays always come hand in hand with secondary effects stemming from either reduction of essential proteins or elevated temperature. In our approach, the former was countered by known information about the targeting signal of the proteins obtained. For example, complex IV subunits down-regulated in this study are known to be imported by the presequence pathway, and could therefore be disregarded as potential TIM22 substrates. To counter the secondary effects from repressive temperature, careful selection was made with respect to the control mitochondria while performing the labelling. This ensured that the pleiotropic effect of elevated temperature, which lead to an overall reduction in protein levels, was taken into account for all time points. 


\subsubsection{Turnover rate of Tim23 and Tim17 is low}

An interesting observation from the MS results was the absence of translocase subunit substrates. Tim23 levels seemed to not be affected in the Tim22 mutant strain. Unfortunately, Tim17 was not detected, most likely due to low sequence coverage or inaccuracy during quantification. While this was surprising, given that some of the carrier proteins were very strongly down-regulated indicating the severity of the ts phenotype, it could be that since Tim23-Tim17 are essential core proteins of the TIM23 complex, their turnover is not that fast. Therefore, once assembled into the TIM23 complex, they are stable and not constantly exchanged. Moreover, it was recently reported that hTim23 and hTim17b (Tim23 and Tim17 in humans) levels were not down-regulated upon knockout or knock-down of human TIM22 complex subunits (Kang et al., 2017) (Pacheu-Grau and Callegari et al., 2018). Additionally, it was demonstrated that the relative stability of proteins involved in mitochondrial protein biogenesis and turnover, including proteins involved in import, folding, maturation and degradation, remained static under different growth conditions (Morgenstern et al., 2017). This indicates that the protein import machinery forms a part of the housekeeping system of mitochondria, such that their functioning remains constantly steady. On the other hand, carrier proteins were described to almost double in copy number on change of carbon source for growth from glucose to glycerol. This points to the dynamic nature of the carrier protein turnover. Therefore, the turnover rate of a potential TIM22 substrate could be governing its extent of downregulation in the Tim22 mutant strain. Indeed, this also implies that, like Tim23 and Tim17, other four or six transmembrane proteins might be TIM22 substrates but are not downregulated due to a low turnover.

\subsubsection{Offbeat import pathways into mitochondria}

Initial import and assembly results of $\mathrm{Mpc1}, \mathrm{Mpc2}$ and $\mathrm{Mpc} 3$ point to them being substrates of the TIM22 complex (data not shown). This is surprising, since they do not belong to the traditional family of carrier proteins with six transmembrane domains. The presence of 2, 3 and 3 transmembrane domains in Mpc1, Mpc2 and Mpc3 respectively makes them atypical TIM22 substrates.

Exceptions within a typical import pathway are not uncommon. Outer membrane proteins typically only require channels in the outer membrane for their insertion. However, for the import of Om45, an OM protein, both the TOM and the TIM23 complexes are involved, after which it has been suggested to be imported into the OM with the help of the MIM machinery (Song et al., 2014) (Wenz et al., 2014). In another study, it was described that the mature part of a presequence-containing protein provides sensitivity to $\Delta \Psi$, an 
exception to the established principle that positive charges on the presequence are important for $\Delta \Psi$-driven import of the protein (Schendzielorz et al., 2017). Moreover, OXA machinery in the IM was typically assigned for import of mitochondria-encoded proteins into the IM. However, based on a recent study, it has also been implicated in being involved in import of numerous nuclear-encoded proteins (Stiller et al., 2016). Therefore, new information is constantly being obtained regarding the substrates and mechanisms of import pathways that are thought to be well-established. This could be a response of the organelle to adapt to the ever-evolving cellular physiology.

In summary, the TIM22 complex is required for the import of four and six transmembrane domain-containing IM proteins, belonging either to the translocases or the carrier family of proteins. Additionally, it is also potentially required for the import of proteins with atypical membrane domains. Extensive biochemical analysis will help in understanding the extent to which these smaller proteins depend on the typical TIM22 complex pathway for their import. 


\section{Summary and Future Perspectives}

Eukaryotic cells are dependent on mitochondria for ATP production, apoptosis and various metabolic pathways. To maintain properly functioning mitochondria, import of proteins synthesised in cytosol to mitochondria is imperative. Dedicated machineries are present in mitochondria to ensure correct import of proteins. In this study, we deal with the TOM, TIM23, TIM22 and the PAM complexes, as well as the translocation intermediate TOM-TIM23 supercomplex.

In the first part of this study, new supercomplex forming proteins were generated. Jac1sfGFP emerged as the most promising protein. The presence of a sfGFP domain at the Cterminus also allowed for the in vivo generation of the supercomplex. Additionally, elution of the TIM23 complex with SUMOstar protease provided specificity to the isolation approach. Isolated complex could be observed as particles after subjecting them to negative staining followed by EM. However, the sample prepared was heterogeneous, most likely due to the presence of detergent micelles as background contamination and possible degradation of the isolated complex. Application of density gradient based fixation and detergent exchange methods improved the sample quality. The yield of the preparation has to be improved for single particle analysis in the future.

From the cross-linking analysis, we could observe that the presequence translocase machinery is dynamic in nature, with structural rearrangements taking place within the subunits during import of a protein. Specifically, interactions within the (i) TOM and TIM23 complexes, such as Tim21-Tom22, Tim23-Tom40 and Tim23-Tom5, (ii) TIM23 complex subunits, such as Tim21-Mgr2, Tim21-Tim50 and Tim23 ${ }^{\mathrm{IMS}}$ interactions, and (iii) PAM complex subunits, such as Hsp70-Tim44, Pam16-Tim44, Pam16-Pam18, indicate that the mitochondrial presequence import pathway involves cross-talk between all the components involved for efficient import of protein. This acute response of the translocase subunits to a translocating protein will be an essential subject of future studies. Mutations within the cross-linking sites followed by biochemical analysis will help in determining the extent of importance of these sites for efficient protein interaction. 
In the second part of this thesis, the TIM22 complex and its substrates were studied. A Tim22 temperature sensitive strain was successfully employed for identification of additional substrates of the TIM22 complex. Quantitative mass spectrometry was applied following labelling of mitochondria from cells grown at repressive temperature for different time points. Putative substrates obtained were confirmed using assembly studies in the mutant Tim22 strain. Carrier family proteins such as Crc1, Odc1, Hem25 and Yhm2 were confirmed as TIM22 substrates. Moreover, uncharacterised proteins YPR011C and YFR045W were also verified. Remarkably, mitochondrial pyruvate carrier (MPC) subunits, which are two and three transmembrane domain proteins, were also affected in the Tim22 mutant strain. Import studies of these proteins will shed some light on the topological requirements of an inner membrane protein to be a TIM22 complex substrate. 


\section{Bibliography}

Abe, Y., Shodai, T., Muto, T., Mihara, K., Torii, H., Nishikawa, S., Endo, T., and Kohda, D. (2000). Structural basis of presequence recognition by the mitochondrial protein import receptor Tom20. Cell 100, 551-560.

Adam, A., Endres, M., Sirrenberg, C., Lottspeich, F., Neupert, W., and Brunner, M. (1999). Tim9, a new component of the TIM22.54 translocase in mitochondria. Embo J. 18, 313319.

Ahting, U., Thun, C., Hegerl, R., Typke, D., Nargang, F.E., Neupert, W., and Nussberger, S. (1999). The TOM core complex: the general protein import pore of the outer membrane of mitochondria. J. Cell Biol. 147, 959-968.

Albrecht, R., Rehling, P., Chacinska, A., Brix, J., Cadamuro, S.A., Volkmer, R., Guiard, B., Pfanner, N., and Zeth, K. (2006). The Tim21 binding domain connects the preprotein translocases of both mitochondrial membranes. EMBO Rep. 7, 1233-1238.

Alconada, A., Kübrich, M., Moczko, M., Hönlinger, A., and Pfanner, N. (1995). The mitochondrial receptor complex: the small subunit Mom8b/lsp6 supports association of receptors with the general insertion pore and transfer of preproteins. Molecular and Cellular Biology 15, 6196-6205.

Alder, N.N., Sutherland, J., Buhring, A.I., Jensen, R.E., and Johnson, A.E. (2008). Mol Biol Cell 2008 Alder. 1-12.

Alkhaja, A.K., Jans, D.C., Nikolov, M., Vukotic, M., Lytovchenko, O., Ludewig, F., Schliebs, W., Riedel, D., Urlaub, H., Jakobs, S., et al. (2012). MINOS1 is a conserved component of mitofilin complexes and required for mitochondrial function and cristae organization. Mol. Biol. Cell 23, 247-257.

Allison, D.S., and Schatz, G. (1986). Artificial mitochondrial presequences. Proc. Natl. Acad. Sci. U.S.a. 83, 9011-9015.

Andersson, S.G., Zomorodipour, A., Andersson, J.O., Sicheritz-Pontén, T., Alsmark, U.C., Podowski, R.M., Näslund, A.K., Eriksson, A.S., Winkler, H.H., and Kurland, C.G. (1998). The genome sequence of Rickettsia prowazekii and the origin of mitochondria. Nature 396, 133-140.

Ast, T., Michaelis, S., and Schuldiner, M. (2016). The Protease Ste24 Clears Clogged Translocons. Cell 164, 103-114.

Backes, S., Hess, S., Boos, F., Woellhaf, M.W., Gödel, S., Jung, M., Mühlhaus, T., and Herrmann, J.M. (2018). Tom70 enhances mitochondrial preprotein import efficiency by binding to internal targeting sequences. J. Cell Biol. 217, 1369-1382.

Bajaj, R., Jaremko, Ł., Jaremko, M., Becker, S., and Zweckstetter, M. (2014a). Molecular basis of the dynamic structure of the TIM23 complex in the mitochondrial intermembrane space. Structure 22, 1501-1511.

Bajaj, R., Munari, F., Becker, S., and Zweckstetter, M. (2014b). Interaction of the intermembrane space domain of Tim23 protein with mitochondrial membranes. Journal of Biological Chemistry 289, 34620-34626. 
Banerjee, R., Gladkova, C., Mapa, K., Witte, G., and Mokranjac, D. (2015). Protein translocation channel of mitochondrial inner membrane and matrix-exposed import motor communicate via two-domain coupling protein. eLife 2015;4: e11897.

Bauer, M.F., Sirrenberg, C., Neupert, W., and Brunner, M. (1996). Role of Tim23 as voltage sensor and presequence receptor in protein import into mitochondria. Cell 87,33 41.

Bausewein, T., Mills, D.J., Langer, J.D., Nitschke, B., Nussberger, S., and Kühlbrandt, W. (2017). Cryo-EM Structure of the TOM Core Complex from Neurospora crassa. Cell 170, 693-700.e697.

Becker, T., Böttinger, L., and Pfanner, N. (2012). Mitochondrial protein import: from transport pathways to an integrated network. Trends Biochem. Sci. 37, 85-91.

Becker, T., Pfannschmidt, S., Guiard, B., Stojanovski, D., Milenkovic, D., Kutik, S., Pfanner, N., Meisinger, C., and Wiedemann, N. (2008). Biogenesis of the mitochondrial TOM complex: Mim1 promotes insertion and assembly of signal-anchored receptors. J. Biol. Chem. 283, 120-127.

Belenkiy, R., Haefele, A., Eisen, M.B., and Wohlrab, H. (2000). The yeast mitochondrial transport proteins: new sequences and consensus residues, lack of direct relation between consensus residues and transmembrane helices, expression patterns of the transport protein genes, and protein-protein interactions with other proteins. Biochim. Biophys. Acta 1467, 207-218.

Bender, T., Pena, G., and Martinou, J.-C. (2015). Regulation of mitochondrial pyruvate uptake by alternative pyruvate carrier complexes. Embo J. 34, 911-924.

Benz, R. (1994). Permeation of hydrophilic solutes through mitochondrial outer membranes: review on mitochondrial porins. Biochim. Biophys. Acta 1197, 167-196.

Blom, J., Kübrich, M., Rassow, J., Voos, W., Dekker, P.J., Maarse, A.C., Meijer, M., and Pfanner, N. (1993). The essential yeast protein MIM44 (encoded by MPI1) is involved in an early step of preprotein translocation across the mitochondrial inner membrane. Molecular and Cellular Biology 13, 7364-7371.

Bornhövd, C., Vogel, F., Neupert, W., and Reichert, A.S. (2006). Mitochondrial membrane potential is dependent on the oligomeric state of F1F0-ATP synthase supracomplexes. J. Biol. Chem. 281, 13990-13998.

Botelho, S.C., Osterberg, M., Reichert, A.S., Yamano, K., Björkholm, P., Endo, T., Heijne, von, G., and Kim, H. (2011). TIM23-mediated insertion of transmembrane a-helices into the mitochondrial inner membrane. Embo J. 30, 1003-1011.

Brandner, K., Rehling, P., and Truscott, K.N. (2005). The Carboxyl-terminal Third of the Dicarboxylate Carrier Is Crucial for Productive Association with the Inner Membrane Twinpore Translocase. Journal of Biological Chemistry 280, 6215-6221.

Brix, J., Dietmeier, K., and Pfanner, N. (1997). Differential recognition of preproteins by the purified cytosolic domains of the mitochondrial import receptors Tom20, Tom22, and Tom70. J. Biol. Chem. 272, 20730-20735.

Bukau, B., and Horwich, A.L. (1998). The Hsp70 and Hsp60 chaperone machines. Cell 92, 351-366. 
Burnette, W.N. (1981). "Western blotting": electrophoretic transfer of proteins from sodium dodecyl sulfate--polyacrylamide gels to unmodified nitrocellulose and radiographic detection with antibody and radioiodinated protein A. Anal. Biochem. 112, 195-203.

Chacinska, A., Rehling, P., Guiard, B., Frazier, A.E., Schulze-Specking, A., Pfanner, N., Voos, W., and Meisinger, C. (2003). Mitochondrial translocation contact sites: separation of dynamic and stabilizing elements in formation of a TOM-TIM-preprotein supercomplex. Embo J. 22, 5370-5381.

Chacinska, A., Koehler, C.M., Milenkovic, D., Lithgow, T., and Pfanner, N. (2009). Importing mitochondrial proteins: machineries and mechanisms. Cell 138, 628-644.

Chacinska, A., Lind, M., Frazier, A.E., Dudek, J., Meisinger, C., Geissler, A., Sickmann, A., Meyer, H.E., Truscott, K.N., Guiard, B., et al. (2005). Mitochondrial presequence translocase: switching between TOM tethering and motor recruitment involves Tim21 and Tim17. Cell 120, 817-829.

Chacinska, A., Pfannschmidt, S., Wiedemann, N., Kozjak, V., Sanjuán Szklarz, L.K., Schulze-Specking, A., Truscott, K.N., Guiard, B., Meisinger, C., and Pfanner, N. (2004). Essential role of Mia40 in import and assembly of mitochondrial intermembrane space proteins. Embo J. 23, 3735-3746.

Chacinska, A., van der Laan, M., Mehnert, C.S., Guiard, B., Mick, D.U., Hutu, D.P., Truscott, K.N., Wiedemann, N., Meisinger, C., Pfanner, N., et al. (2010). Distinct forms of mitochondrial TOM-TIM supercomplexes define signal-dependent states of preprotein sorting. Molecular and Cellular Biology 30, 307-318.

Chae, P.S., Rasmussen, S.G.F., Rana, R.R., Gotfryd, K., Chandra, R., Goren, M.A., Kruse, A.C., Nurva, S., Loland, C.J., Pierre, Y., et al. (2010). Maltose-neopentyl glycol (MNG) amphiphiles for solubilization, stabilization and crystallization of membrane proteins. Nat. Methods 7, 1003-1008.

Chae, P.S., Rasmussen, S.G.F., Rana, R.R., Gotfryd, K., Kruse, A.C., Manglik, A., Cho, K.H., Nurva, S., Gether, U., Guan, L., et al. (2012). A new class of amphiphiles bearing rigid hydrophobic groups for solubilization and stabilization of membrane proteins. Chemistry 18, 9485-9490.

Clapham, D.E. (2007). Calcium signaling. Cell 131, 1047-1058.

Craig, E.A. (2018). Hsp70 at the membrane: driving protein translocation. BMC Biol. 16, 11.

Curran, S.P., Leuenberger, D., Schmidt, E., and Koehler, C.M. (2002). The role of the Tim8p-Tim13p complex in a conserved import pathway for mitochondrial polytopic inner membrane proteins. J. Cell Biol. 158, 1017-1027.

D'Silva, P.D., Schilke, B., Walter, W., Andrew, A., and Craig, E.A. (2003). J protein cochaperone of the mitochondrial inner membrane required for protein import into the mitochondrial matrix. Proc. Natl. Acad. Sci. U.S.a. 100, 13839-13844.

D'Silva, P.R., Schilke, B., Hayashi, M., and Craig, E.A. (2008). Interaction of the J-protein heterodimer Pam18/Pam16 of the mitochondrial import motor with the translocon of the inner membrane. Mol. Biol. Cell 19, 424-432.

D'Silva, P., Liu, Q., Walter, W., and Craig, E.A. (2004). Regulated interactions of mtHsp70 with Tim44 at the translocon in the mitochondrial inner membrane. Nat. Struct. Mol. Biol. 
Dekker, P.J., Keil, P., Rassow, J., Maarse, A.C., Pfanner, N., and Meijer, M. (1993). Identification of MIM23, a putative component of the protein import machinery of the mitochondrial inner membrane. FEBS Lett. 330, 66-70.

Dekker, P.J., Martin, F., Maarse, A.C., Bömer, U., Muller, H., Guiard, B., Meijer, M., Rassow, J., and Pfanner, N. (1997). The Tim core complex defines the number of mitochondrial translocation contact sites and can hold arrested preproteins in the absence of matrix Hsp70-Tim44. Embo J. 16, 5408-5419.

Demishtein-Zohary, K., Günsel, U., Marom, M., Banerjee, R., Neupert, W., Azem, A., and Mokranjac, D. (2017). Role of Tim17 in coupling the import motor to the translocation channel of the mitochondrial presequence translocase. Elife 6.

Demishtein-Zohary, K., Marom, M., Neupert, W., Mokranjac, D., and Azem, A. (2015). GxxxG Motifs hold the TIM23 Complex Together. Febs J.

Denkert, N., Schendzielorz, A.B., Barbot, M., Versemann, L., Richter, F., Rehling, P., and Meinecke, M. (2017). Cation selectivity of the presequence translocase channel Tim23 is crucial for efficient protein import. Elife 6.

Dietmeier, K., Hönlinger, A., Bömer, U., Dekker, P.J., Eckerskorn, C., Lottspeich, F., Kübrich, M., and Pfanner, N. (1997). Tom5 functionally links mitochondrial preprotein receptors to the general import pore. Nature 388, 195-200.

Donzeau, M., Káldi, K., Adam, A., Paschen, S., Wanner, G., Guiard, B., Bauer, M.F., Neupert, W., and Brunner, M. (2000). Tim23 links the inner and outer mitochondrial membranes. Cell 101, 401-412.

Dörr, J.M., Scheidelaar, S., Koorengevel, M.C., Dominguez, J.J., Schäfer, M., van Walree, C.A., and Killian, J.A. (2016). The styrene-maleic acid copolymer: a versatile tool in membrane research. Eur. Biophys. J. 45, 3-21.

Dudek, J., Rehling, P., and van der Laan, M. (2013). Mitochondrial protein import: common principles and physiological networks. Biochim. Biophys. Acta 1833, 274-285.

Dufay, J.N., Fernández-Murray, J.P., and McMaster, C.R. (2017). SLC25 Family Member Genetic Interactions Identify a Role for HEM25 in Yeast Electron Transport Chain Stability. G3 (Bethesda) 7, 1861-1873.

Emtage, J.L., and Jensen, R.E. (1993). MAS6 encodes an essential inner membrane component of the yeast mitochondrial protein import pathway. J. Cell Biol. 122, 10031012.

Endo, T., Yamano, K., and Kawano, S. (2011). Structural insight into the mitochondrial protein import system. BBA - Biomembranes 1808, 955-970.

Endres, M., Neupert, W., and Brunner, M. (1999). Transport of the ADP/ATP carrier of mitochondria from the TOM complex to the TIM22.54 complex. Embo J. 18, 3214-3221.

Esaki, M., Kanamori, T., Nishikawa, S.-I., Shin, I., Schultz, P.G., and Endo, T. (2003). Tom40 protein import channel binds to non-native proteins and prevents their aggregation. Nat. Struct. Biol. 10, 988-994.

Ferramosca, A., and Zara, V. (2013). Biogenesis of mitochondrial carrier proteins: 
Molecular mechanisms of import into mitochondria. Bioch Biophys Acta 1833, 494-502.

Fölsch, H., Guiard, B., Neupert, W., and Stuart, R.A. (1996). Internal targeting signal of the BCS1 protein: a novel mechanism of import into mitochondria. EMBO J vol. 15 no. 3, pp 479-487.

Frazier, A.E., Dudek, J., Guiard, B., Voos, W., Li, Y., Lind, M., Meisinger, C., Geissler, A., Sickmann, A., Meyer, H.E., et al. (2004). Pam16 has an essential role in the mitochondrial protein import motor. Nat. Struct. Mol. Biol. 11, 226-233.

Frey, S., and Görlich, D. (2015). The Xenopus laevis Atg4B Protease: Insights into Substrate Recognition and Application for Tag Removal from Proteins Expressed in Proand Eukaryotic Hosts. PLoS ONE 10, e0125099.

Friedman, J.R., and Nunnari, J. (2014). Mitochondrial form and function. Nature 505, 335343.

Gakh, O., Cavadini, P., and Isaya, G. (2002). Mitochondrial processing peptidases. Biochim. Biophys. Acta 1592, 63-77.

Gebert, M., Schrempp, S.G., Mehnert, C.S., Heißwolf, A.K., Oeljeklaus, S., leva, R., Bohnert, M., Malsburg, von der, K., Wiese, S., Kleinschroth, T., et al. (2012). Mgr2 promotes coupling of the mitochondrial presequence translocase to partner complexes. J. Cell Biol. 197, 595-604.

Gebert, N., Chacinska, A., Wagner, K., Guiard, B., Koehler, C.M., Rehling, P., Pfanner, N., and Wiedemann, N. (2008). Assembly of the three small Tim proteins precedes docking to the mitochondrial carrier translocase. EMBO Rep. 9, 548-554.

Gebert, N., Gebert, M., Oeljeklaus, S., Malsburg, von der, K., Stroud, D.A., Kulawiak, B., Wirth, C., Zahedi, R.P., Dolezal, P., Wiese, S., et al. (2011). Dual Function of Sdh3 in the Respiratory Chain and TIM22 Protein Translocase of the Mitochondrial Inner Membrane. Molecular Cell 44, 811-818.

Geissler, A., Chacinska, A., Truscott, K.N., Wiedemann, N., Brandner, K., Sickmann, A., Meyer, H.E., Meisinger, C., Pfanner, N., and Rehling, P. (2002). The mitochondrial presequence translocase: an essential role of Tim50 in directing preproteins to the import channel. Cell 111, 507-518.

Gevorkyan-Airapetov, L., Zohary, K., Popov-Čeleketić, D., Mapa, K., Hell, K., Neupert, W., Azem, A., and Mokranjac, D. (2009). Interaction of Tim23 with Tim50 Is essential for protein translocation by the mitochondrial TIM23 complex. J. Biol. Chem. 284, 4865-4872.

Gietz, R.D., and Woods, R.A. (2002). Transformation of yeast by lithium acetate/singlestranded carrier DNA/polyethylene glycol method. Meth. Enzymol. 350, 87-96.

Glick, B.S., Brandt, A., Cunningham, K., Müller, S., Hallberg, R.L., and Schatz, G. (1992). Cytochromes $\mathrm{c} 1$ and $\mathrm{b} 2$ are sorted to the intermembrane space of yeast mitochondria by a stop-transfer mechanism. Cell 69, 809-822.

Gold, V.A.M., leva, R., Walter, A., Pfanner, N., van der Laan, M., and Kühlbrandt, W. (2014). Visualizing active membrane protein complexes by electron cryotomography. Nature Communications 5, 4129.

Gray, M.W. (1998). Rickettsia, typhus and the mitochondrial connection. Nature 396, 109110. 
Gray, M.W., Cedergren, R., Abel, Y., and Sankoff, D. (1989). On the evolutionary origin of the plant mitochondrion and its genome. Proc. Natl. Acad. Sci. U.S.a. 86, 2267-2271.

Gray, M.W., Sankoff, D., and Cedergren, R.J. (1984). On the evolutionary descent of organisms and organelles: a global phylogeny based on a highly conserved structural core in small subunit ribosomal RNA. Nucleic Acids Res. 12, 5837-5852.

Green, D.R., and Reed, J.C. (1998). Mitochondria and apoptosis. Science 281, 13091312.

Greene, M.K., Maskos, K., and Landry, S.J. (1998). Role of the J-domain in the cooperation of Hsp40 with Hsp70. Proc. Natl. Acad. Sci. U.S.a. 95, 6108-6113.

Grimm, M., Zimniak, T., Kahraman, A., and Herzog, F. (2015). xVis: a web server for the schematic visualization and interpretation of crosslink-derived spacial restraints. Nucleic Acids Res. 43, W362-9.

Hackenbrock, C.R. (1968). Chemical and physical fixation of isolated mitochondria in lowenergy and high-energy states. Proc. Natl. Acad. Sci. U.S.a. 61, 598-605.

Harner, M., Körner, C., Walther, D., Mokranjac, D., Kaesmacher, J., Welsch, U., Griffith, J., Mann, M., Reggiori, F., and Neupert, W. (2011). The mitochondrial contact site complex, a determinant of mitochondrial architecture. Embo J. 30, 4356-4370.

Hartl, F.U., and Hayer-Hartl, M. (2002). Molecular chaperones in the cytosol: from nascent chain to folded protein. Science 295, 1852-1858.

Hauer, F., Gerle, C., Fischer, N., Oshima, A., Shinzawa-Itoh, K., Shimada, S., Yokoyama, K., Fujiyoshi, Y., and Stark, H. (2015). GraDeR: Membrane Protein Complex Preparation for Single-Particle Cryo-EM. Structure 23, 1769-1775.

Heijne, von, G., Steppuhn, J., and Herrmann, R.G. (1989). Domain structure of mitochondrial and chloroplast targeting peptides. Eur. J. Biochem. 180, 535-545.

Herrmann, J.M., Westermann, B., and Neupert, W. (2001). Analysis of protein-protein interactions in mitochondria by coimmunoprecipitation and chemical cross-linking. Methods Cell Biol. 65, 217-230.

Hill, K., Model, K., Ryan, M.T., Dietmeier, K., Martin, F., Wagner, R., and Pfanner, N. (1998). Tom40 forms the hydrophilic channel of the mitochondrial import pore for preproteins [see comment]. Nature 395, 516-521.

Hink, M.A., Griep, R.A., Borst, J.W., van Hoek, A., Eppink, M.H., Schots, A., and Visser, A.J. (2000). Structural dynamics of green fluorescent protein alone and fused with a single chain Fv protein. J. Biol. Chem. 275, 17556-17560.

Hoppins, S., Collins, S.R., Cassidy-Stone, A., Hummel, E., Devay, R.M., Lackner, L.L., Westermann, B., Schuldiner, M., Weissman, J.S., and Nunnari, J. (2011). A mitochondrialfocused genetic interaction map reveals a scaffold-like complex required for inner membrane organization in mitochondria. J. Cell Biol. 195, 323-340.

Horst, M., Oppliger, W., Rospert, S., Schönfeld, H.J., Schatz, G., and Azem, A. (1997). Sequential action of two hsp70 complexes during protein import into mitochondria. Embo J. 16, 1842-1849.

Hsu, J.-L., Huang, S.-Y., Chow, N.-H., and Chen, S.-H. (2003). Stable-isotope dimethyl 
labeling for quantitative proteomics. Anal. Chem. 75, 6843-6852.

Huh, W.-K., Falvo, J.V., Gerke, L.C., Carroll, A.S., Howson, R.W., Weissman, J.S., and O'Shea, E.K. (2003). Global analysis of protein localization in budding yeast. Nature 425, 686-691.

Hulett, J.M., Lueder, F., Chan, N.C., Perry, A.J., Wolynec, P., Likić, V.A., Gooley, P.R., and Lithgow, T. (2008). The transmembrane segment of Tom20 is recognized by Mim1 for docking to the mitochondrial TOM complex. J. Mol. Biol. 376, 694-704.

Hutu, D.P., Guiard, B., Chacinska, A., Becker, D., Pfanner, N., Rehling, P., and van der Laan, M. (2008). Mitochondrial protein import motor: differential role of Tim44 in the recruitment of Pam17 and J-complex to the presequence translocase. Mol. Biol. Cell 19, 2642-2649.

leva, R., Heißwolf, A.K., Gebert, M., Vögtle, F.-N., Wollweber, F., Mehnert, C.S., Oeljeklaus, S., Warscheid, B., Meisinger, C., van der Laan, M., et al. (2013). Mitochondrial inner membrane protease promotes assembly of presequence translocase by removing a carboxy-terminal targeting sequence. Nature Communications 4, 2853.

leva, R., Schrempp, S.G., Opaliński, L., Wollweber, F., Höß, P., Heißwolf, A.K., Gebert, M., Zhang, Y., Guiard, B., Rospert, S., et al. (2014). Mgr2 functions as lateral gatekeeper for preprotein sorting in the mitochondrial inner membrane. Molecular Cell 56, 641-652.

losefson, O., Levy, R., Marom, M., Slutsky-Leiderman, O., and Azem, A. (2007). The Pam18/Tim14-Pam16/Tim16 complex of the mitochondrial translocation motor: the formation of a stable complex from marginally stable proteins. Protein Sci. 16, 316-322.

Izawa, T., Park, S-H., Zhao, L., Hartl, F.U., and Neupert, W. (2017). Cytosolic Protein Vms1 Links Ribosome Quality Control to Mitochondrial and Cellular Homeostasis. Cell 171, 890-903.

Jiang, J., Maes, E.G., Taylor, A.B., Wang, L., Hinck, A.P., Lafer, E.M., and Sousa, R. $(2007)$. Structural basis of $\mathrm{J}$ cochaperone binding and regulation of Hsp70. Molecular Cell $28,422-433$.

Jiang, J., Prasad, K., Lafer, E.M., and Sousa, R. (2005). Structural basis of interdomain communication in the Hsc70 chaperone. Molecular Cell 20, 513-524.

Jores, T., Lawatscheck, J., Beke, V., Franz-Wachtel, M., Yunoki, K., Fitzgerald, J.C., Macek, B., Endo, T., Kalbacher, H., Buchner, J., et al. (2018). Cytosolic Hsp70 and Hsp40 chaperones enable the biogenesis of mitochondrial $\beta$-barrel proteins. J. Cell Biol.

Josyula, R., Jin, Z., Fu, Z., and Sha, B. (2006). Crystal structure of yeast mitochondrial peripheral membrane protein Tim44p C-terminal domain. J. Mol. Biol. 359, 798-804.

Kang, P.J., Ostermann, J., Shilling, J., Neupert, W., Craig, E.A., and Pfanner, N. (1990). Requirement for hsp70 in the mitochondrial matrix for translocation and folding of precursor proteins. Nature 348, 137-143.

Kang, Y., Stroud, D.A., Baker, M.J., De Souza, D.P., Frazier, A.E., Liem, M., Tull, D., Mathivanan, S., McConville, M.J., Thorburn, D.R., et al. (2017). Sengers SyndromeAssociated Mitochondrial Acylglycerol Kinase Is a Subunit of the Human TIM22 Protein Import Complex. Molecular Cell 67, 457-470.e5.

Kastner, B., Fischer, N., Golas, M.M., Sander, B., Dube, P., Boehringer, D., Hartmuth, K., 
Deckert, J., Hauer, F., Wolf, E., et al. (2008). GraFix: sample preparation for singleparticle electron cryomicroscopy. Nat. Methods 5, 53-55.

Keeling, P.J., and Archibald, J.M. (2008). Organelle evolution: what's in a name? Curr. Biol. 18, R345-R347.

Kerscher, O., Holder, J., Srinivasan, M., Leung, R.S., and Jensen, R.E. (1997). The Tim54p-Tim22p complex mediates insertion of proteins into the mitochondrial inner membrane. J. Cell Biol. 139, 1663-1675.

Kerscher, O., Sepuri, N.B., and Jensen, R.E. (2000). Tim18p is a new component of the Tim54p-Tim22p translocon in the mitochondrial inner membrane. Mol. Biol. Cell 11, 103116.

Kiebler, M., Pfaller, R., Söllner, T., Griffiths, G., Horstmann, H., Pfanner, N., and Neupert, W. (1990). Identification of a mitochondrial receptor complex required for recognition and membrane insertion of precursor proteins. Nature 348, 610-616.

Koehler, C.M., Jarosch, E., Tokatlidis, K., Schmid, K., Schweyen, R.J., and Schatz, G. (1998a). Import of mitochondrial carriers mediated by essential proteins of the intermembrane space. Science 279, 369-373.

Koehler, C.M., Leuenberger, D., Merchant, S., Renold, A., Junne, T., and Schatz, G. (1999). Human deafness dystonia syndrome is a mitochondrial disease. Proc. Natl. Acad. Sci. U.S.a. 96, 2141-2146.

Koehler, C.M., Merchant, S., Oppliger, W., Schmid, K., Jarosch, E., Dolfini, L., Junne, T., Schatz, G., and Tokatlidis, K. (1998b). Tim9p, an essential partner subunit of Tim10p for the import of mitochondrial carrier proteins. Embo J. 17, 6477-6486.

Koehler, C.M., Murphy, M.P., Bally, N.A., Leuenberger, D., Oppliger, W., Dolfini, L., Junne, T., Schatz, G., and Or, E. (2000). Tim18p, a new subunit of the TIM22 complex that mediates insertion of imported proteins into the yeast mitochondrial inner membrane. Molecular and Cellular Biology 20, 1187-1193.

Koll, H., Guiard, B., Rassow, J., Ostermann, J., Horwich, A.L., Neupert, W., and Hartl, F.U. (1992). Antifolding activity of hsp60 couples protein import into the mitochondrial matrix with export to the intermembrane space. Cell 68, 1163-1175.

Kovermann, P., Truscott, K.N., Guiard, B., Rehling, P., Sepuri, N.B., Müller, H., Jensen, R.E., Wagner, R., and Pfanner, N. (2002). Tim22, the essential core of the mitochondrial protein insertion complex, forms a voltage-activated and signal-gated channel. Molecular Cell 9, 363-373.

Kozany, C., Mokranjac, D., Sichting, M., Neupert, W., and Hell, K. (2004). The J domainrelated cochaperone Tim16 is a constituent of the mitochondrial TIM23 preprotein translocase. Nat. Struct. Mol. Biol. 11, 234-241.

Krimmer, T., Rassow, J., Kunau, W.H., Voos, W., and Pfanner, N. (2000). Mitochondrial protein import motor: the ATPase domain of matrix Hsp70 is crucial for binding to Tim44, while the peptide binding domain and the carboxy-terminal segment play a stimulatory role. Molecular and Cellular Biology 20, 5879-5887.

Kronidou, N.G., Oppliger, W., Bolliger, L., Hannavy, K., Glick, B.S., Schatz, G., and Horst, M. (1994). Dynamic interaction between Isp45 and mitochondrial hsp70 in the protein import system of the yeast mitochondrial inner membrane. Proc. Natl. Acad. Sci. U.S.a. 
Krüger, V., Becker, T., Becker, L., Montilla-Martinez, M., Ellenrieder, L., Vögtle, F.-N., Meyer, H.E., Ryan, M.T., Wiedemann, N., Warscheid, B., et al. (2017). Identification of new channels by systematic analysis of the mitochondrial outer membrane. J. Cell Biol. $216,3485-3495$.

Kulawiak, B., Höpker, J., Gebert, M., Guiard, B., Wiedemann, N., and Gebert, N. (2013). The mitochondrial protein import machinery has multiple connections to the respiratory chain. Biochim. Biophys. Acta 1827, 612-626.

Kunji, E.R.S. (2004). The role and structure of mitochondrial carriers. FEBS Lett. 564, 239-244.

Kunji, E.R.S., and Harding, M. (2003). Projection structure of the atractyloside-inhibited mitochondrial ADP/ATP carrier of Saccharomyces cerevisiae. J. Biol. Chem. 278, 3698536988.

Kübrich, M., Keil, P., Rassow, J., Dekker, P.J., Blom, J., Meijer, M., and Pfanner, N. (1994). The polytopic mitochondrial inner membrane proteins MIM17 and MIM23 operate at the same preprotein import site. FEBS Lett. 349, 222-228.

la Cruz, de, L., Bajaj, R., Becker, S., and Zweckstetter, M. (2010). The intermembrane space domain of Tim23 is intrinsically disordered with a distinct binding region for presequences. Protein Sci. 19, 2045-2054.

Lackey, S.W.K., Taylor, R.D., Go, N.E., Wong, A., Sherman, E.L., and Nargang, F.E. (2014). Evidence supporting the $19 \beta$-strand model for Tom40 from cysteine scanning and protease site accessibility studies. Journal of Biological Chemistry 289, 21640-21650.

Laemmli, U.K. (1970). Cleavage of structural proteins during the assembly of the head of bacteriophage T4. Nature 227, 680-685.

Laloraya, S., Gambill, B.D., and Craig, E.A. (1994). A role for a eukaryotic GrpE-related protein, Mge1p, in protein translocation. Proc. Natl. Acad. Sci. U.S.a. 91, 6481-6485.

Lee, C.M., Sedman, J., Neupert, W., and Stuart, R.A. (1999). The DNA helicase, Hmi1p, is transported into mitochondria by a C-terminal cleavable targeting signal. J. Biol. Chem. 274, 20937-20942.

Li, Y., Dudek, J., Guiard, B., Pfanner, N., Rehling, P., and Voos, W. (2004). The presequence translocase-associated protein import motor of mitochondria. Pam16 functions in an antagonistic manner to Pam18. J. Biol. Chem. 279, 38047-38054.

Lill, R., Hoffmann, B., Molik, S., Pierik, A.J., Rietzschel, N., Stehling, O., Uzarska, M.A., Webert, H., Wilbrecht, C., and Mühlenhoff, U. (2012). The role of mitochondria in cellular iron-sulfur protein biogenesis and iron metabolism. Biochim. Biophys. Acta 1823, 14911508.

Lohret, T.A., Jensen, R.E., and Kinnally, K.W. (1997). Tim23, a protein import component of the mitochondrial inner membrane, is required for normal activity of the multiple conductance channel, MCC. J. Cell Biol. 137, 377-386.

Lytovchenko O. (2012) Structural and Functional Analysis of the Mitochondrial Presequence Translocase. PhD Thesis. 
Lytovchenko, O., Melin, J., Schulz, C., Kilisch, M., Hutu, D.P., and Rehling, P. (2013). Signal recognition initiates reorganization of the presequence translocase during protein import. Embo J. 32, 886-898.

Maarse, A.C., Blom, J., Keil, P., Pfanner, N., and Meijer, M. (1994). Identification of the essential yeast protein MIM17, an integral mitochondrial inner membrane protein involved in protein import. FEBS Lett. 349, 215-221.

Malhotra, K., Sathappa, M., Landin, J.S., Johnson, A.E., and Alder, N.N. (2013). Nat Struct Mol Biol 2013 Malhotra. Nature Publishing Group 20, 965-972.

Malsburg, von der, K., Müller, J.M., Bohnert, M., Oeljeklaus, S., Kwiatkowska, P., Becker, T., Loniewska-Lwowska, A., Wiese, S., Rao, S., Milenkovic, D., et al. (2011). Dual role of mitofilin in mitochondrial membrane organization and protein biogenesis. Dev. Cell 21, 694-707.

Mapa, K., Sikor, M., Kudryavtsev, V., Waegemann, K., Kalinin, S., Seidel, C.A.M., Neupert, W., Lamb, D.C., and Mokranjac, D. (2010). The conformational dynamics of the mitochondrial Hsp70 chaperone. Molecular Cell 38, 89-100.

Margolin, W. (2005). FtsZ and the division of prokaryotic cells and organelles. Nat. Rev. Mol. Cell Biol. 6, 862-871.

Margulis, L. (1970). Origin of Eukaryotic Cells. Yale University Press.

Marom, M., Dayan, D., Demishtein-Zohary, K., Mokranjac, D., Neupert, W., and Azem, A. (2011). Direct interaction of mitochondrial targeting presequences with purified components of the TIM23 protein complex. Journal of Biological Chemistry 286, 4380943815.

Marom, M., Safonov, R., Amram, S., Avneon, Y., Nachliel, E., Gutman, M., Zohary, K., Azem, A., and Tsfadia, Y. (2009). Interaction of the Tim44 C-terminal domain with negatively charged phospholipids. Biochemistry 48, 11185-11195.

Martijn, J., Vosseberg, J., Guy, L., Offre, P., and Ettema, T.J.G. (2018). Deep mitochondrial origin outside the sampled alphaproteobacteria. Nature 557, 101-105.

Martin, J., Mahlke, K., and Pfanner, N. (1991). Role of an energized inner membrane in mitochondrial protein import. Delta psi drives the movement of presequences. J. Biol. Chem. 266, 18051-18057.

Martin, W., Hoffmeister, M., Rotte, C., and Henze, K. (2001). An overview of endosymbiotic models for the origins of eukaryotes, their ATP-producing organelles (mitochondria and hydrogenosomes), and their heterotrophic lifestyle. Biol. Chem. 382, 1521-1539.

Martinez-Caballero, S., Grigoriev, S.M., Herrmann, J.M., Campo, M.L., and Kinnally, K.W. (2007). Tim17p regulates the twin pore structure and voltage gating of the mitochondrial protein import complex TIM23. J. Biol. Chem. 282, 3584-3593.

Mayer, M.P. (2013). Hsp70 chaperone dynamics and molecular mechanism. Trends Biochem. Sci. 38, 507-514.

Mehnert, C.S., Rampelt, H., Gebert, M., Oeljeklaus, S., Schrempp, S.G., Kochbeck, L., Guiard, B., Warscheid, B., and van der Laan, M. (2014). The mitochondrial ADP/ATP carrier associates with the inner membrane presequence translocase in a stoichiometric 
manner. Journal of Biological Chemistry 289, 27352-27362.

Meinecke, M., Wagner, R., Kovermann, P., Guiard, B., Mick, D.U., Hutu, D.P., Voos, W., Truscott, K.N., Chacinska, A., Pfanner, N., et al. (2006). Tim50 maintains the permeability barrier of the mitochondrial inner membrane. Science 312, 1523-1526.

Meisinger, C., Pfanner, N., and Truscott, K.N. (2006). Isolation of yeast mitochondria. Methods Mol. Biol. 313, 33-39.

Melin, J., Kilisch, M., Neumann, P., Lytovchenko, O., Gomkale, R., Schendzielorz, A., Schmidt, B., Liepold, T., Ficner, R., Jahn, O., et al. (2015). A presequence-binding groove in Tom70 supports import of Mdl1 into mitochondria. Biochim. Biophys. Acta 1850, 18501859.

Mitchell, P. (1961). Coupling of phosphorylation to electron and hydrogen transfer by a chemi-osmotic type of mechanism. Nature 191, 144-148.

Model, K., Meisinger, C., and Kuehlbrandt, W. (2008). Cryo-Electron Microscopy Structure of a Yeast Mitochondrial Preprotein Translocase. J. Mol. Biol. 383, 1049-1057.

Mokranjac, D., Bourenkov, G., Hell, K., Neupert, W., and Groll, M. (2006). Structure and function of Tim14 and Tim16, the $\mathrm{J}$ and J-like components of the mitochondrial protein import motor. Embo J. 25, 4675-4685.

Mokranjac, D., Sichting, M., Popov-Čeleketić, D., Mapa, K., Gevorkyan-Airapetov, L., Zohary, K., Hell, K., Azem, A., and Neupert, W. (2009). Role of Tim50 in the transfer of precursor proteins from the outer to the inner membrane of mitochondria. Mol. Biol. Cell 20, 1400-1407.

Morgenstern, M., Stiller, S.B., Lübbert, P., Peikert, C.D., Dannenmaier, S., Drepper, F., Weill, U., Höß, P., Feuerstein, R., Gebert, M., et al. (2017). Definition of a High-

Confidence Mitochondrial Proteome at Quantitative Scale. Cell Rep 19, 2836-2852.

Moro, F., Okamoto, K., Donzeau, M., Neupert, W., and Brunner, M. (2002). Mitochondrial protein import: molecular basis of the ATP-dependent interaction of MtHsp70 with Tim44. J. Biol. Chem. 277, 6874-6880.

Naoé, M., Ohwa, Y., Ishikawa, D., Ohshima, C., Nishikawa, S.-I., Yamamoto, H., and Endo, T. (2004). Identification of Tim40 that mediates protein sorting to the mitochondrial intermembrane space. J. Biol. Chem. 279, 47815-47821.

Nelson, D.R., Felix, C.M., and Swanson, J.M. (1998). Highly conserved charge-pair networks in the mitochondrial carrier family. J. Mol. Biol. 277, 285-308.

Neuhoff, V., Arold, N., Taube, D., and Ehrhardt, W. (1988). Improved staining of proteins in polyacrylamide gels including isoelectric focusing gels with clear background at nanogram sensitivity using Coomassie Brilliant Blue G-250 and R-250. Electrophoresis 9, 255-262.

Neupert, W., and Herrmann, J.M. (2007). Translocation of proteins into mitochondria. Annu. Rev. Biochem. 76, 723-749.

Neupert, W., and Schatz, G. (1981). How proteins are transported into mitochondria.

O'Brien, R.L., and Brierley, G. (1965). Compartmentation of heart mitochondria. I.

Permeability characteristics of isolated beef heart mitochondria. J. Biol. Chem. 240, 4527- 
Osellame, L.D., Blacker, T.S., and Duchen, M.R. (2012). Cellular and molecular mechanisms of mitochondrial function. Best Pract. Res. Clin. Endocrinol. Metab. 26, 711723.

Paschen, S.A., Rothbauer, U., Káldi, K., Bauer, M.F., Neupert, W., and Brunner, M. (2000). The role of the TIM8-13 complex in the import of Tim23 into mitochondria. Embo J. 19, 6392-6400.

Pacheu-Grau, D., Callegari, S., Emperador, S., Thompson, K., Aich, A., Topol, S. E., Spencer, E.G., McFarland, R., Ruiz-Pesinin, E., Torkamani, A., Taylor, R.W., Montoya, J., and Rehling, P. (2018). Mutations of the mitochondrial carrier translocase channel subunit TIM22 cause early-onset mitochondrial myopathy. Human Molecular Genetics, ddy305.

Paschen, S.A., Waizenegger, T., Stan, T., Preuss, M., Cyrklaff, M., Hell, K., Rapaport, D., and Neupert, W. (2003). Evolutionary conservation of biogenesis of beta-barrel membrane proteins. Nature 426, 862-866.

Paumard, P., Vaillier, J., Coulary, B., Schaeffer, J., Soubannier, V., Mueller, D.M., Brèthes, D., di Rago, J.-P., and Velours, J. (2002). The ATP synthase is involved in generating mitochondrial cristae morphology. Embo J. 21, 221-230.

Pebay-Peyroula, E., Dahout-Gonzalez, C., Kahn, R., Trézéguet, V., Lauquin, G.J.-M., and Brandolin, G. (2003). Structure of mitochondrial ADP/ATP carrier in complex with carboxyatractyloside. Nature 426, 39-44.

Perocchi, F., Jensen, L.J., Gagneur, J., Ahting, U., Mering, von, C., Bork, P., Prokisch, H., and Steinmetz, L.M. (2006). Assessing systems properties of yeast mitochondria through an interaction map of the organelle. PLoS Genet. 2, e170.

Peroutka, R.J., Elshourbagy, N., Piech, T., and Butt, T.R. (2008). Enhanced protein expression in mammalian cells using engineered SUMO fusions: secreted phospholipase A2. Protein Sci. 17, 1586-1595.

Pédelacq, J.-D., Cabantous, S., Tran, T., Terwilliger, T.C., and Waldo, G.S. (2006). Engineering and characterization of a superfolder green fluorescent protein. Nat. Biotechnol. 24, 79-88.

Pfanner, N., Hartl, F.U., and Neupert, W. (1988). Import of proteins into mitochondria: a multi-step process. Eur. J. Biochem. 175, 205-212.

Pfanner, N., Hartl, F.U., Guiard, B., and Neupert, W. (1987a). Mitochondrial precursor proteins are imported through a hydrophilic membrane environment. Eur. J. Biochem. 169, 289-293.

Pfanner, N., Hoeben, P., Tropschug, M., and Neupert, W. (1987b). The carboxyl-terminal two-thirds of the ADP/ATP carrier polypeptide contains sufficient information to direct translocation into mitochondria. J. Biol. Chem. 262, 14851-14854.

Pfanner, N., Tropschug, M., and Neupert, W. (1987c). Mitochondrial protein import: nucleoside triphosphates are involved in conferring import-competence to precursors. Cell $49,815-823$.

Popov-Celeketić, J., Waizenegger, T., and Rapaport, D. (2008). Mim1 functions in an oligomeric form to facilitate the integration of Tom20 into the mitochondrial outer 
membrane. J. Mol. Biol. 376, 671-680.

Popov-Čeleketić, D., Mapa, K., Neupert, W., and Mokranjac, D. (2008). Active remodelling of the TIM23 complex during translocation of preproteins into mitochondria. Embo J. 27, $1469-1480$.

Qian, X., Gebert, M., Höpker, J., Yan, M., Li, J., Wiedemann, N., van der Laan, M., Pfanner, N., and Sha, B. (2011). Structural basis for the function of Tim50 in the mitochondrial presequence translocase. J. Mol. Biol. 411, 513-519.

Qiu, J., Wenz, L.-S., Zerbes, R.M., Oeljeklaus, S., Bohnert, M., Stroud, D.A., Wirth, C., Ellenrieder, L., Thornton, N., Kutik, S., et al. (2013). Coupling of mitochondrial import and export translocases by receptor-mediated supercomplex formation. Cell 154, 596-608.

Rahman, B., Kawano, S., Yunoki-Esaki, K., Anzai, T., and Endo, T. (2014). NMR analyses on the interactions of the yeast Tim50 C-terminal region with the presequence and Tim50 core domain. FEBS Lett. 588, 678-684.

Ramesh, A., Peleh, V., Martinez-Caballero, S., Wollweber, F., Sommer, F., van der Laan, M., Schroda, M., Alexander, R.T., Campo, M.L., and Herrmann, J.M. (2016). A disulfide bond in the TIM23 complex is crucial for voltage gating and mitochondrial protein import. J. Cell Biol. 214, 417-431.

Rassow, J., Guiard, B., Wienhues, U., Herzog, V., Hartl, F.U., and Neupert, W. (1989). Translocation arrest by reversible folding of a precursor protein imported into mitochondria. A means to quantitate translocation contact sites. J. Cell Biol. 109, 14211428.

Rehling, P., Brandner, K., and Pfanner, N. (2004). Mitochondrial import and the twin-pore translocase. Nat. Rev. Mol. Cell Biol. 5, 519-530.

Rehling, P., Model, K., Brandner, K., Kovermann, P., Sickmann, A., Meyer, H.E., Kühlbrandt, W., Wagner, R., Truscott, K.N., and Pfanner, N. (2003). Protein insertion into the mitochondrial inner membrane by a twin-pore translocase. Science $299,1747-1751$.

Reinhold, R., Krüger, V., Meinecke, M., Schulz, C., Schmidt, B., Grunau, S.D., Guiard, B., Wiedemann, N., van der Laan, M., Wagner, R., et al. (2012). The channel-forming Sym1 protein is transported by the TIM23 complex in a presequence-independent manner. Molecular and Cellular Biology 32, 5009-5021.

Roise, D., Horvath, S.J., Tomich, J.M., Richards, J.H., and Schatz, G. (1986). A chemically synthesized pre-sequence of an imported mitochondrial protein can form an amphiphilic helix and perturb natural and artificial phospholipid bilayers. Embo J. 5, 13271334.

Rowley, N., Prip-Buus, C., Westermann, B., Brown, C., Schwarz, E., Barrell, B., and Neupert, W. (1994). Mdj1p, a novel chaperone of the DnaJ family, is involved in mitochondrial biogenesis and protein folding. Cell 77, 249-259.

Ryan, K.R., Menold, M.M., Garrett, S., and Jensen, R.E. (1994). SMS1, a high-copy suppressor of the yeast mas 6 mutant, encodes an essential inner membrane protein required for mitochondrial protein import. Mol. Biol. Cell 5, 529-538.

Ryan, M.T., Muller, H., and Pfanner, N. (1999). Functional staging of ADP/ATP carrier translocation across the outer mitochondrial membrane. J. Biol. Chem. 274, 20619 20627. 
Ryan, M.T., Voos, W., and Pfanner, N. (2001). Assaying protein import into mitochondria. Methods Cell Biol. 65, 189-215.

Saiki, R.K., Scharf, S., Faloona, F., Mullis, K.B., Horn, G.T., Erlich, H.A., and Arnheim, N. (1985). Enzymatic amplification of beta-globin genomic sequences and restriction site analysis for diagnosis of sickle cell anemia. Science 230, 1350-1354.

Sambrook, J., and Russell, D.W. (2001). Molecular Cloning (CSHL Press).

Saraste, M. (1999). Oxidative phosphorylation at the fin de siècle. Science 283, 14881493.

Saraste, M., and Walker, J.E. (1982). Internal sequence repeats and the path of polypeptide in mitochondrial ADP/ATP translocase. FEBS Lett. 144, 250-254.

Schägger, H., and von Jagow, G. (1991). Blue native electrophoresis for isolation of membrane protein complexes in enzymatically active form. Anal Biochem 199, 223-231.

Scharf, S.J., Horn, G.T., and Erlich, H.A. (1986). Direct cloning and sequence analysis of enzymatically amplified genomic sequences. Science 233, 1076-1078.

Schatz, G., and Butow, R.A. (1983). How are proteins imported into mitochondria? Cell 32, 316-318.

Schatz, G., and Dobberstein, B. (1996). Common principles of protein translocation across membranes. Science 271, 1519-1526.

Schendzielorz, A.B., Schulz, C., Lytovchenko, O., Clancy, A., Guiard, B., leva, R., van der Laan, M., and Rehling, P. (2017). Two distinct membrane potential-dependent steps drive mitochondrial matrix protein translocation. J. Cell Biol. 216, 83-92.

Schilke, B.A., Hayashi, M., and Craig, E.A. (2012). Genetic analysis of complex interactions among components of the mitochondrial import motor and translocon in Saccharomyces cerevisiae. Genetics 190, 1341-1353.

Schiller, D., Cheng, Y.C., Liu, Q., Walter, W., and Craig, E.A. (2008). Residues of Tim44 involved in both association with the translocon of the inner mitochondrial membrane and regulation of mitochondrial Hsp70 tethering. Molecular and Cellular Biology 28, 44244433.

Schleyer, M., and Neupert, W. (1985). Transport of proteins into mitochondria:

translocational intermediates spanning contact sites between outer and inner membranes. Cell 43, 339-350.

Schlossmann, J., Dietmeier, K., Pfanner, N., and Neupert, W. (1994). Specific recognition of mitochondrial preproteins by the cytosolic domain of the import receptor MOM72. J.

Biol. Chem. 269, 11893-11901.

Schmidt, S., Strub, A., Röttgers, K., Zufall, N., and Voos, W. (2001). The two mitochondrial heat shock proteins 70 , Ssc1 and Ssq1, compete for the cochaperone Mge1. J. Mol. Biol. 313, 13-26.

Schneider, H.C., Berthold, J., Bauer, M.F., Dietmeier, K., Guiard, B., Brunner, M., and Neupert, W. (1994). Mitochondrial Hsp70/MIM44 complex facilitates protein import. Nature $371,768-774$. 
Schneider, H.C., Westermann, B., Neupert, W., and Brunner, M. (1996). The nucleotide exchange factor MGE exerts a key function in the ATP-dependent cycle of mt-Hsp70Tim44 interaction driving mitochondrial protein import. Embo J. 15, 5796-5803.

Schneider, G., Sjöling, S., Wallin, E., Wrede, P., Glaser, E., and von Heijne, G. (1998). Feature extraction from endopeptidase cleavage sites in mitochondrial targeting peptides. Proteins 30, 49-60.

Schulz, C., and Rehling, P. (2014). Remodelling of the active presequence translocase drives motor-dependent mitochondrial protein translocation. Nature Communications 5 , 4349 .

Schulz, C., Lytovchenko, O., Melin, J., Chacinska, A., Guiard, B., Neumann, P., Ficner, R., Jahn, O., Schmidt, B., and Rehling, P. (2011). Tim50's presequence receptor domain is essential for signal driven transport across the TIM23 complex. J. Cell Biol. 195, 643656.

Schulz, C., Schendzielorz, A., and Rehling, P. (2015). Unlocking the presequence import pathway. Trends in Cell Biology 1-11.

Schwaiger, M., Herzog, V., and Neupert, W. (1987). Characterization of translocation contact sites involved in the import of mitochondrial proteins. J. Cell Biol. 105, 235-246.

Shadel, G.S., and Horvath, T.L. (2015). Mitochondrial ROS signaling in organismal homeostasis. Cell 163, 560-569.

Shiota, T., Imai, K., Qiu, J., Hewitt, V.L., Tan, K., Shen, H.-H., Sakiyama, N., Fukasawa, Y., Hayat, S., Kamiya, M., et al. (2015). Molecular architecture of the active mitochondrial protein gate. Science $349,1544-1548$.

Shiota, T., Mabuchi, H., Tanaka-Yamano, S., Yamano, K., and Endo, T. (2011). In vivo protein-interaction mapping of a mitochondrial translocator protein Tom22 at work.

Proceedings of the National Academy of Sciences 108, 15179-15183.

Sickmann, A., Reinders, J., Wagner, Y., Joppich, C., Zahedi, R., Meyer, H.E., Schönfisch, B., Perschil, I., Chacinska, A., Guiard, B., et al. (2003). The proteome of Saccharomyces cerevisiae mitochondria. Proc. Natl. Acad. Sci. U.S.a. 100, 13207-13212.

Sikor, M., Mapa, K., Voithenberg, von, L.V., Mokranjac, D., and Lamb, D.C. (2013). Realtime observation of the conformational dynamics of mitochondrial Hsp70 by spFRET.

Embo J. 32, 1639-1649.

Sikorski, R.S., and Hieter, P. (1989). A system of shuttle vectors and yeast host strains designed for efficient manipulation of DNA in Saccharomyces cerevisiae. Genetics 122, 19-27.

Sirrenberg, C., Bauer, M.F., Guiard, B., Neupert, W., and Brunner, M. (1996). Import of carrier proteins into the mitochondrial inner membrane mediated by Tim22. Nature 384 , 582-585.

Sirrenberg, C., Endres, M., Fölsch, H., Stuart, R.A., Neupert, W., and Brunner, M. (1998). Carrier protein import into mitochondria mediated by the intermembrane proteins Tim10/Mrs11 and Tim12/Mrs5. Nature 391, 912-915.

Song, J., Tamura, Y., Yoshihisa, T., and Endo, T. (2014). A novel import route for an Nanchor mitochondrial outer membrane protein aided by the TIM23 complex. EMBO Rep. 
$15,670-677$.

Söllner, T., Rassow, J., Wiedmann, M., Schlossmann, J., Keil, P., Neupert, W., and Pfanner, N. (1992). Mapping of the protein import machinery in the mitochondrial outer membrane by crosslinking of translocation intermediates. Nature $355,84-87$.

Stan, T., Ahting, U., Dembowski, M., Kunkele, K.P., Nussberger, S., Neupert, W., and Rapaport, D. (2000). Recognition of preproteins by the isolated TOM complex of mitochondria. Embo J. 19, 4895-4902.

Stark, H., and Chari, A. (2016). Sample preparation of biological macromolecular assemblies for the determination of high-resolution structures by cryo-electron microscopy. Microscopy (Oxf) 65, 23-34.

Stiller, S.B., Höpker, J., Oeljeklaus, S., Schütze, C., Schrempp, S.G., Vent-Schmidt, J., Horvath, S.E., Frazier, A.E., Gebert, N., van der Laan, M., et al. (2016). Mitochondrial OXA Translocase Plays a Major Role in Biogenesis of Inner-Membrane Proteins. Cell Metab. 23, 901-908.

Swain, J.F., Dinler, G., Sivendran, R., Montgomery, D.L., Stotz, M., and Gierasch, L.M. (2007). Hsp70 chaperone ligands control domain association via an allosteric mechanism mediated by the interdomain linker. Molecular Cell 26, 27-39.

Szyperski, T., Pellecchia, M., Wall, D., Georgopoulos, C., and Wüthrich, K. (1994). NMR structure determination of the Escherichia coli DnaJ molecular chaperone: secondary structure and backbone fold of the $\mathrm{N}$-terminal region (residues 2-108) containing the highly conserved J domain. Proc. Natl. Acad. Sci. U.S.a. 91, 11343-11347.

Takeda, S., and McKay, D.B. (1996). Kinetics of peptide binding to the bovine $70 \mathrm{kDa}$ heat shock cognate protein, a molecular chaperone. Biochemistry 35, 4636-4644.

Tamura, Y., Harada, Y., Shiota, T., Yamano, K., Watanabe, K., Yokota, M., Yamamoto, H., Sesaki, H., and Endo, T. (2009). Tim23-Tim50 pair coordinates functions of translocators and motor proteins in mitochondrial protein import. J. Cell Biol. 184, 129141.

Taylor, A.B., Smith, B.S., Kitada, S., Kojima, K., Miyaura, H., Otwinowski, Z., Ito, A., and Deisenhofer, J. (2001). Crystal structures of mitochondrial processing peptidase reveal the mode for specific cleavage of import signal sequences. Structure 9, 615-625.

Terziyska, N., Lutz, T., Kozany, C., Mokranjac, D., Mesecke, N., Neupert, W., Herrmann, J.M., and Hell, K. (2005). Mia40, a novel factor for protein import into the intermembrane space of mitochondria is able to bind metal ions. FEBS Lett. 579, 179-184.

Timmis, J.N., Ayliffe, M.A., Huang, C.Y., and Martin, W. (2004). Endosymbiotic gene transfer: organelle genomes forge eukaryotic chromosomes. Nat Rev Genet 5, 123-135.

Ting, S.-Y., Schilke, B.A., Hayashi, M., and Craig, E.A. (2014). Architecture of the TIM23 inner mitochondrial translocon and interactions with the matrix import motor. Journal of Biological Chemistry 289, 28689-28696.

Ting, S.-Y., Yan, N.L., Schilke, B.A., and Craig, E.A. (2017). Dual interaction of scaffold protein Tim44 of mitochondrial import motor with channel-forming translocase subunit Tim23. Elife 6.

Todisco, S., Di Noia, M.A., Castegna, A., Lasorsa, F.M., Paradies, E., and Palmieri, F. 
(2014). The Saccharomyces cerevisiae gene YPR011c encodes a mitochondrial transporter of adenosine 5'-phosphosulfate and 3'-phospho-adenosine 5'-phosphosulfate. Biochim. Biophys. Acta 1837, 326-334.

Truscott, K.N., Kovermann, P., Geissler, A., Merlin, A., Meijer, M., Driessen, A.J., Rassow, J., Pfanner, N., and Wagner, R. (2001). A presequence- and voltage-sensitive channel of the mitochondrial preprotein translocase formed by Tim23. Nat. Struct. Biol. 8, 10741082.

Truscott, K.N., Voos, W., Frazier, A.E., Lind, M., Li, Y., Geissler, A., Dudek, J., Müller, H., Sickmann, A., Meyer, H.E., et al. (2003). A J-protein is an essential subunit of the presequence translocase-associated protein import motor of mitochondria. J. Cell Biol. 163, 707-713.

van der Laan, M., Bohnert, M., Wiedemann, N., and Pfanner, N. (2012). Role of MINOS in mitochondrial membrane architecture and biogenesis. Trends in Cell Biology 22, 185-192.

van der Laan, M., Chacinska, A., Lind, M., Perschil, I., Sickmann, A., Meyer, H.E., Guiard, B., Meisinger, C., Pfanner, N., and Rehling, P. (2005). Pam17 is required for architecture and translocation activity of the mitochondrial protein import motor. Molecular and Cellular Biology 25, 7449-7458.

van der Laan, M., Meinecke, M., Dudek, J., Hutu, D.P., Lind, M., Perschil, I., Guiard, B., Wagner, R., Pfanner, N., and Rehling, P. (2007). Motor-free mitochondrial presequence translocase drives membrane integration of preproteins. Nat. Cell Biol. 9, 1152-1159.

van der Laan, M., Schrempp, S.G., and Pfanner, N. (2013). Voltage-coupled conformational dynamics of mitochondrial protein-import channel. Nature Publishing Group 20, 915-917.

van der Laan, M., Wiedemann, N., Mick, D.U., Guiard, B., Rehling, P., and Pfanner, N. (2006). A role for Tim21 in membrane-potential-dependent preprotein sorting in mitochondria. Curr. Biol. 16, 2271-2276.

van Wilpe, S., Ryan, M.T., Hill, K., Maarse, A.C., Meisinger, C., Brix, J., Dekker, P.J., Moczko, M., Wagner, R., Meijer, M., et al. (1999). Tom22 is a multifunctional organizer of the mitochondrial preprotein translocase. Nature 401, 485-489.

Vogel, F., Bornhövd, C., Neupert, W., and Reichert, A.S. (2006). Dynamic subcompartmentalization of the mitochondrial inner membrane. J. Cell Biol. 175, 237-247.

Vögtle, F.-N., Wortelkamp, S., Zahedi, R.P., Becker, D., Leidhold, C., Gevaert, K., Kellermann, J., Voos, W., Sickmann, A., Pfanner, N., et al. (2009). Global analysis of the mitochondrial $\mathrm{N}$-proteome identifies a processing peptidase critical for protein stability. Cell 139, 428-439.

Waegemann, K., Popov-Čeleketić, D., Neupert, W., Azem, A., and Mokranjac, D. (2015). Cooperation of TOM and TIM23 Complexes during Translocation of Proteins into Mitochondria. J. Mol. Biol. 427, 1075-1084.

Wagner, K., Gebert, N., Guiard, B., Brandner, K., Truscott, K.N., Wiedemann, N., Pfanner, N., and Rehling, P. (2008). The Assembly Pathway of the Mitochondrial Carrier Translocase Involves Four Preprotein Translocases. Molecular and Cellular Biology 28, 4251-4260.

Wall, D., Zylicz, M., and Georgopoulos, C. (1994). The NH2-terminal 108 amino acids of 
the Escherichia coli DnaJ protein stimulate the ATPase activity of DnaK and are sufficient for lambda replication. J. Biol. Chem. 269, 5446-5451.

Weidberg, H., and Amon, A. (2018). MitoCPR-A surveillance pathway that protects mitochondria in response to protein import stress. Science 360.

Weiss, C., Oppliger, W., Vergères, G., Demel, R., Jenö, P., Horst, M., de Kruijff, B., Schatz, G., and Azem, A. (1999). Domain structure and lipid interaction of recombinant yeast Tim44. Proc. Natl. Acad. Sci. U.S.a. 96, 8890-8894.

Wenz, L-S., Opalinski, L., Schuler, M-H., Ellenrieder, L., leva, R., Böttinger, L., Qiu, J., van der Laan, M., Wiedemann, N., Guiard, B., Pfanner, N., and Becker, T. (2014). The presequence pathway is involved in protein sorting to the mitochondrial outer membrane. EMBO reports 15 (6), 678-685.

Wiedemann, N., Pfanner, N., and Ryan, M.T. (2001). The three modules of ADP/ATP carrier cooperate in receptor recruitment and translocation into mitochondria. Embo J. 20, 951-960.

Wiedemann, N., and Pfanner, N. (2017). Mitochondrial Machineries for Protein Import and Assembly. Annu. Rev. Biochem. 86, 685-714.

Wiedemann, N., Kozjak, V., Chacinska, A., Schönfisch, B., Rospert, S., Ryan, M.T., Pfanner, N., and Meisinger, C. (2003). Machinery for protein sorting and assembly in the mitochondrial outer membrane. Nature 424, 565-571.

Wiedemann, N., Truscott, K.N., Pfannschmidt, S., Guiard, B., Meisinger, C., and Pfanner, N. (2004). Biogenesis of the protein import channel Tom40 of the mitochondrial outer membrane: intermembrane space components are involved in an early stage of the assembly pathway. J. Biol. Chem. 279, 18188-18194.

Wiedemann, N., van der Laan, M., Hutu, D.P., Rehling, P., and Pfanner, N. (2007). Sorting switch of mitochondrial presequence translocase involves coupling of motor module to respiratory chain. J. Cell Biol. 179, 1115-1122.

Wrobel, L., Sokol, A.M., Chojnacka, M., and Chacinska, A. (2016). The presence of disulfide bonds reveals an evolutionarily conserved mechanism involved in mitochondrial protein translocase assembly. Sci Rep 6, 27484.

Wu, Y., and Sha, B. (2006). Crystal structure of yeast mitochondrial outer membrane translocon member Tom70p. Nat. Struct. Mol. Biol. 13, 589-593.

Yaffe, M.P., and Schatz, G. (1984). Two nuclear mutations that block mitochondrial protein import in yeast. Proc. Natl. Acad. Sci. U.S.a. 81, 4819-4823.

Yamamoto, H., Esaki, M., Kanamori, T., Tamura, Y., Nishikawa, S.-I., and Endo, T. (2002). Tim50 is a subunit of the TIM23 complex that links protein translocation across the outer and inner mitochondrial membranes. Cell 111, 519-528.

Yang, D., Oyaizu, Y., Oyaizu, H., Olsen, G.J., and Woese, C.R. (1985). Mitochondrial origins. Proc. Natl. Acad. Sci. U.S.a. 82, 4443-4447.

Young, J.C., Hoogenraad, N.J., and Hartl, F.U. (2003). Molecular chaperones Hsp90 and Hsp70 deliver preproteins to the mitochondrial import receptor Tom70. Cell 112, 41-50. 


\section{Curriculum Vitae}

\section{Personal Information}

Name: Ridhima Gomkale

Date of birth: 23.07.1990

Place of birth: New Delhi, India

Current Work Address: Institute of Cellular Biochemistry, Humboldtallee 23, 37073,

Göttingen, Germany

E-Mail: ridhima.gomkale@med.uni-goettingen.de

\section{Education}

09/2014-09/2018

PhD thesis in the group of Prof. Dr. Peter Rehling, Institute of Cellular Biochemistry, University Medical Centre Göttingen, Germany

Project: Insights into mitochondrial presequence and carrier import pathways.

09/2013-09/2014 Completion of theoretical and practical segment of the MSc program (IMPRS Molecular Biology)

09/2013-09/2018

MSc/PhD program IMPRS Molecular Biology, Göttingen, Germany

07/2011-06/2013

M.Sc. Biochemistry, DU, New Delhi, India

Thesis: Multiple putative hemoglobin reductases from Chlamydomonas reinhardtii support NO scavenging function of globins.

Supervisor: Prof. Suman Kundu

07/2008-05/2011

B.Sc. (Hons). Biochemistry, DU, New Delhi, India

2007-2008

A.I.S.S.C.E, St. Thomas' School, New Delhi, India 


\section{Publications}

- Gomkale, R.*, Cruz-Zaragoza, L.D.*, Suppanz, I., Guiard, B., Montoya, J., Callegari, S., Pacheu-Grau, D., Warscheid, B. and Peter Rehling. "Defining the Substrate Spectrum of the TIM22 Complex Identifies Pyruvate Carrier Subunits as Unconventional Cargos" Current Biology 30(6) (2020).

- Schendzielorz, A.B., Bragoszewski, P., Naumenko, N., Gomkale, R., Schulz, C., Guiard, B., Chacinska, A. and Rehling, P. "Motor recruitment to the TIM23 channel's lateral gate restricts polypeptide release into the inner membrane" Nature Communications 9, 4028 (2018).

- Melin, J., Kilisch, M., Neumann, P., Lytovchenko, O., Gomkale, R., Schendzielorz, A., Schmidt, B., Liepold, T., Ficner, R., Jahn, O., Rehling, P. and Schulz, C. "A presequencebinding groove in Tom70 supports import of Mdl1 into mitochondria" BBA Molecular Cell Research 1853 (8) (2015). 